\title{
Spectral functions of low-dimensional quantum systems
}

\section{Dissertation}

zur Erlangung des mathematisch-naturwissenschaftlichen Doktorgrades

\author{
„Doctor rerum naturalium“" \\ der Georg-August-Universität Göttingen
}

im Promotionsprogramm ProPhys

der Georg-August University School of Science (GAUSS)

vorgelegt von

Piet Dargel

aus Bremen

Göttingen, 2012 
$\underline{\text { Betreuungsausschuss }}$

Prof. Dr. Thomas Pruschke,

Institut für theoretische Physik, Georg-August-Universität Göttingen

Dr. Martin Wenderoth,

IV. Physikalisches Institut, Georg-August-Universität Göttingen

Prof. Dr. Kurt Schönhammer,

Institut für theoretische Physik, Georg-August-Universität Göttingen

Mitglieder der Prüfungskommission

Referent: Prof. Dr. Thomas Pruschke,

Institut für theoretische Physik, Georg-August-Universität Göttingen

Korreferent: Prof. Dr. Reinhard Noack,

Fachbereich Physik, Philipps-Universität Marburg

2. Korreferent: Prof. Dr. Holger Fehske, Institut für Physik, Ernst Moritz Arndt Universität Greifswald

Weitere Mitglieder der Prüfungskommission

Prof. Dr. Claus Ropers,

Courant Forschungszentrum, Institut für Materialphysik, Georg-August-Universität Göttingen

Prof. Dr. Philipp Gegenwart,

I. Physikalisches Institut, Georg-August-Universität Göttingen

Prof. Dr. Markus Münzenberg,

I. Physikalisches Institut, Georg-August-Universität Göttingen

Prof. Dr. Stefan Kehrein,

Institut für theoretische Physik, Georg-August-Universität Göttingen

Tag der mündlichen Prüfung: 


\section{Acknowledgment}

This thesis could have never been accomplished without the help of many people.

At first, of course a big thank you to Thomas Pruschke who already shared his ideas and wisdom with me starting in the undergrad courses at the beginning of my physics education and during my diploma thesis. He gave me the opportunity to work on this $\mathrm{PhD}$ project and gave me a lot of freedom to choose the topics and methods myself, but never forgot to give me helping comments and ideas. Thank you a lot for this.

Then of course a big thank you to all the members of my group who supported me with discussions about various physics related topics (but also non-physics related topics) in the office, at the coffee table or in the cafeteria. Special thanks to Andreas Honecker who helped me a lot with his physical insight and to my officemate and long-standing companion Ansgar Kalz. The office has always been a place of fun, vivid discussions and company and not of displeasure and isolation. Also my previous officemates, Oliver Bodensiek and Peter Wächter, are not be forgotten as they also contributed a lot to this fortunate situation.

A big thank you also to my national and international collaborators who contributed to the different projects. From our university I feel very lucky to acknowledge the work of my experimental physics colleagues, Henning Prüser, Alexander Weismann, Martin Wenderoth and Rainer G. Ulbrich, who carried out the fascinating experiments with the STM. The complicated, tedious and time-consuming production of this high resolution STM data cannot be appreciated enough. I always enjoyed the discussions about the interpretation of the measured data and learned a lot about the difficulties to compare theoretical models to the real world. From Marburg I have to thank Reinhard Noack for sharing his knowledge about the DMRG with me and his support to publish my idea of the adaptive Lanczos method with the DMRG. Robert Peters from Göttingen, respectively Kyoto now, deserves a big thank you for providing me the basis to my DMRG code. A big thank you also to my colleagues from Munich Ulrich Schollwöck, Anton Wöllert and I.P. McCulloch from Brisbane, Australia who worked with me together on the MPS Lanczos algorithm. 
This thesis would have also not be in this form without the remarks of Ansgar Kalz, Oliver Bodensiek, Birgit Muncke, Alexander Tiegel and Ebad Kamil who carefully read the manuscript. All mistakes and spelling errors have been inserted afterwards by myself again.

Also I have to say thank you to the Deutsche Forschungsgemeinschaft for financing this project via SFB 602, Komplexe Strukturen in kondensierter Materie. Furthermore, I would like to thank Jürgen Holm and the GWDG for technical support during the last years.

Last but not least I would like to thank my family for 30 years of support. I always felt encouraged and backed up, no matter what I wanted to do in my life. I know that this is not standard and therefore I appreciate it a lot. Finally a big thank you to Birgit for four wonderful years and hopefully many more. So let me say it to all of you in the words of Shakespeare ...

"I can no other answer make, but, thanks, and thanks." ( Twelfth Night, William Shakespeare , 1602 )

Piet Dargel, October 2012 


\section{Authors note}

At the end of this thesis a complete list of all references with detailed information (all authors, title, url) is given in alphabetical order of the labels. The labels for all references are constructed by the initials of up to four authors of the work and a two-digit number representing the year of publication or appearance. If more than one reference is cited at the same location the labels are ordered alphabetically to make it easier to find them all in the list at the end rather than in chronological order.

The references which were published within this thesis are:

[PWD ${ }^{+11]}$ Prüser, H., Wenderoth, M., Dargel, P. b., Weismann, A., Peters, R., Pruschke, T. and Ulbrich, R. G., Long-range Kondo signature of a single magnetic impurity, Nat. Phys., 7(3), 203 (2011), URL http://dx.doi.org/10.1038/nphys1876.

$\left[\mathrm{DHP}^{+} 11\right]$ Dargel, P. E., Honecker, A., Peters, R., Noack, R. M. and Pruschke, T. , Adaptive Lanczos-vector method for dynamic properties within the density matrix renormalization group, Physical Review B, 83, 161104 (2011), URL http://dx.doi.org/10. 1103/PhysRevB.83.161104.

[DWH $\left.{ }^{+} 12\right]$ Dargel, P. E., Wöllert, A., Honecker, A., McCulloch, I. P., Schollwöck, U. and Pruschke, T., Lanczos algorithm with matrix product states for dynamical correlation functions, Physical Review B, 85, 205119 (2012), URL http://dx.doi.org/10. 1103/PhysRevB.85.205119. 


\section{Table of contents}

Chapter 1 Introduction 1

1.1. Emergent phenomena in strongly correlated electron systems . . . . . . . . 1

1.2. Spectral functions . . . . . . . . . . . . . . . . . . . . . . . . . . . . . . . . . . .

1.3. Methods . . . . . . . . . . . . . . . . . . . 5

1.4. Long range Kondo signatures . . . . . . . . . . . . . . . . . . 7

1.5. Dynamical spin structure factor . . . . . . . . . . . . . . . . . . . . . . . . . . . . . . . . .

Outline . . . . . . . . . . . . . . . . . . . 10

Chapter 2 Many-body quantum physics 11

2.1. Physical states and Hilbert space . . . . . . . . . . . . . . . . . 11

2.2. Expectation values and static correlation functions . . . . . . . . . . . . . . . 12

2.3. Dynamic correlation functions . . . . . . . . . . . . . . . . . . . . . . . . . . 13

2.3.1. Definition and Green's function . . . . . . . . . . . . . . . 13

2.3.2. Spectral functions . . . . . . . . . . . . . . . . . 14

$\begin{array}{lll}\text { Chapter } 3 & \text { Computational tools } & 17\end{array}$

3.1. Exact diagonalization techniques . . . . . . . . . . . . . . . . 18

3.1.1. Exact diagonalization . . . . . . . . . . . . . . . . . . . . . . . . . . . . . 18

3.1.2. Lanczos algorithm . . . . . . . . . . . . . . . . . . . . . . . . . . . . . . . . . . . . . .

3.2. Renormalization group . . . . . . . . . . . . . . . . . 22

3.2.1. Introduction . . . . . . . . . . . . . . . . . 22

3.2.2. Renormalization group transformation . . . . . . . . . . . . . . 23

3.2.3. Numerical renormalization group . . . . . . . . . . . . . . 24

3.3. Matrix product states and density matrix renormalization group . . . . 31

3.3.1. Introduction . . . . . . . . . . . . . . . . . 31

3.3.2. Formalism of matrix product states . . . . . . . . . . . . . . . . . . . . . . . . . . 32

3.3.3. Ground state search . . . . . . . . . . . . . . . 35

3.3.4. Compression of matrix product states . . . . . . . . . . . 37

3.3.5. Entanglement entropy . . . . . . . . . . . . . . . . . . 38

3.3.6. Subtle differences . . . . . . . . . . . . . . . . . . . . . . . . . . . . . . . . . . . . . . . . .

3.3.7. Spectral functions . . . . . . . . . . . . . . . . . . . . . . . . . . . . . . . .

3.3.8. Numerical renormalization group vs. matrix product states . . . . . 46

$\begin{array}{lll}\text { Chapter } 4 & \text { Long range Kondo signatures } & 49\end{array}$

4.1. The Kondo effect . . . . . . . . . . . . . . . . . . . . . 50 50 
4.2. The single impurity Anderson model and the Kondo model . . . . . . . . . . . . . . . . . . 53

4.2.1. Definition . . . . . . . . . . . . . . . . 53

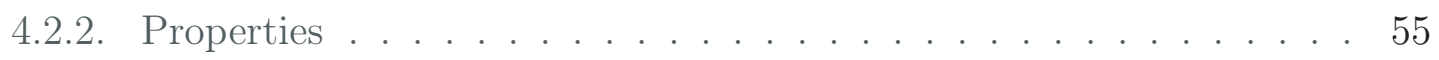

4.3. Experiments . . . . . . . . . . . . . . . . . . . . 61

4.3.1. Scanning tunnelling spectroscopy . . . . . . . . . . . . 61

4.3.2. Kondo signatures from ad-atoms . . . . . . . . . . . . . . 62

4.3.3. Kondo signatures from buried magnetic impurities . . . . . . . . 63

4.4. Simulation . . . . . . . . . . . . . . . . . . . 64

4.4.1. Free Green's function of copper and the focussing effect . . . . . . . 65

4.4.2. Interactions and Kondo physics . . . . . . . . . . . . . . . . 68

4.4.3. Phenomenological expression . . . . . . . . . . . . . . . . 70

4.5. Comparison of simulation and experiment . . . . . . . . . . 73

4.5.1. Local density of states at the surface . . . . . . . . . . . . . . . . . . . . . . . . . . . 73

4.5.2. Kondo temperatures . . . . . . . . . . . . . . . . 74

4.5.3. Phase shift . . . . . . . . . . . . . . . . . . . 75

4.6. Analysis of the data . . . . . . . . . . . . . . . . 75

4.6.1. Effective model . . . . . . . . . . . . . . . . . . 75

4.6.2. Observing a real Kondo signature ? . . . . . . . . . . . . . . . . . . . 77

4.6.3. Measuring the Kondo cloud ? . . . . . . . . . . . . . . . 78

Chapter 5 Dynamic spin structure factor of the one-dimensional $\begin{array}{ll}\text { Heisenberg model } & 81\end{array}$

5.1. Anti-ferromagnetic spin- $\frac{1}{2}$ Heisenberg chain . . . . . . . . . . . . . 82

5.1.1. Ferromagnetic ground state and magnon excitations . . . . . . . . 82

5.1.2. Anti-ferromagnetic chain and spinon excitations . . . . . . . . . 83

5.1.3. Dynamic spin structure factor . . . . . . . . . . . . 86

5.2. Computation with matrix product states . . . . . . . . . . 86

5.2.1. Implementation and entanglement entropy . . . . . . . . . . . . . . 87

5.2.2. Precision and error analysis . . . . . . . . . . . . . . . 87

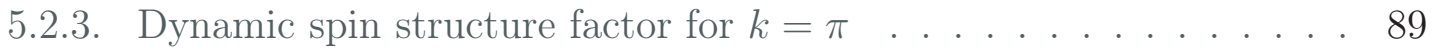

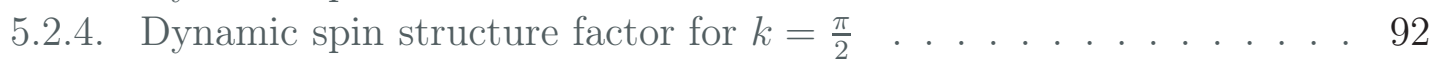

5.2.5. Divergence analysis . . . . . . . . . . . . . . . . . . . . . . . . . . . . . . . . . . . . .

5.3. Comparison to other methods . . . . . . . . . . . . . . . . . 92

5.3.1. Original Lanczos DMRG method . . . . . . . . . . . . 93

5.3.2. Correction vector . . . . . . . . . . . . . . . . . 93

5.3.3. Chebyshev polynomials . . . . . . . . . . . . . . . . . 94

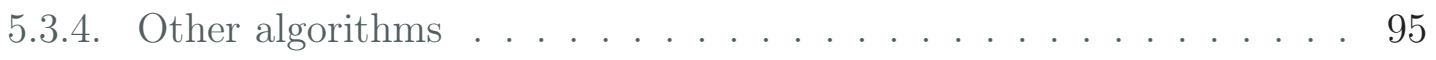

$\begin{array}{lll}\text { Chapter } 6 & \text { Conclusion } & 97\end{array}$

6.1. Long range Kondo signatures . . . . . . . . . . . . . . . . . . . . 97

6.1.1. Retrospective . . . . . . . . . . . . . . . . . . . . . . . . 97

6.1.2. Perspective . . . . . . . . . . . . . . . . . . . 98

6.2. Dynamic spin structure factor of the one-dimensional Heisenberg model . . 100

6.2.1. Retrospective . . . . . . . . . . . . . . 100

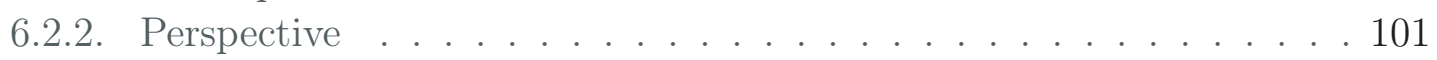




\section{Appendices}

Chapter A Computational details

A.1. Matrix product states . . . . . . . . . . . . . . . . . III

A.1.1. Matrix product state representation of quantum states . . . . . . . III

A.1.2. Hamiltonian as a Matrix Product Operator . . . . . . . . . . . . IV

A.1.3. DMRG ground state search . . . . . . . . . . . . . . IV

A.1.4. Recursive formula for reorthogonalization . . . . . . . . . . . VII

Chapter B Long range Kondo signature IX

B.1. Free Green's function with surface . . . . . . . . . . . . . . . . IX

B.2. Fano line shapes . . . . . . . . . . . . . . . . . . . X

\section{List of references}




\title{
Introduction
}

In Sec. 1.1 this thesis begins with a short description of emergent phenomena in strongly correlated electron systems from which two of them: the Kondo effect and anti-ferromagnetism are subject of this work. Then in Sec. 1.2 the concept of a spectral function is presented which is the physical observable of interest in both of the subjects. Methods to calculate spectral functions for strongly correlated electron systems are listed in Sec. 1.3. The introduction closes with a short description of the two topics of this thesis. ${ }^{1}$

\section{Emergent phenomena in strongly correlated electron systems

\begin{abstract}
"The behavior of large and complex aggregates of elementary particles, it turns out, is not to be understood in terms of a simple extrapolation of the properties of a few particles. Instead, at each level of complexity entirely new properties appear, and the understanding of the new behaviors requires research which I think is as fundamental in its nature as any other."
\end{abstract}

(Article: "More is different", Anderson, 1972,[And72])

In his seminal paper from 1972 Anderson established the concept of emergence in the physics community [And72, Col12]. One major goal of physics is to understand the fundamental laws of nature, attempting to explain all the properties and interactions between the elementary particles. However, regarding many particles new phenomena can emerge that cannot be simply explained by extrapolations from the properties of these single particles [And72]. In this regard physicists started to talk about different behaviour on different scales which can be either, e.g. time, length, energy or number of particles [Col12]. In particular in the field of condensed matter this turned out to be a very successful concept. In classical statistical physics these phenomena emerge mainly at large scales, however for quantum systems the situation can be much more interesting. A very illustrative example is given by Coleman with the comparison of niobium and gold:

\begin{abstract}
"While classical matter develops new forms of behaviour on large scales, the potential for quantum matter to develop emergent properties is far more startling. For instance, similar atoms of niobium and gold, when scaled up to the micron-scale, form crystals with dramatically different properties. Electrons roam free across gold crystals, forming the conducting fluid that gives it lustrous metallic properties. Up to about 30 nanometers, there is little to distinguish copper [sic! ${ }^{2}$ and niobium, but beyond this scale, the electrons in niobium pair up into "Cooper pairs" . By the time we reach the scale of a micron, these pairs congregate by the billions into a pair condensate transforming the crystal into an entirely new metallic state: a superconductor, which conducts without resistance, excludes magnetic fields and has the ability to levitate magnets."
\end{abstract}

(Book: "Introduction to Many Body Physics", Coleman, 2012,[Col12])

\footnotetext{
${ }^{1}$ Any literature cited in this introduction should have an additional 'e.g.' and the comment 'plus references therein'.

${ }^{2}$ It is assumed that the author actually means gold here.
} 
A very interesting subclass of all materials that show emergent new phenomena are the so-called strongly correlated electron systems. In these systems the strong Coulomb interactions between the electrons have to be explicitly taken into account. The regime is characterized by interaction energies that are larger than the kinetic energy of the particles. Prominent examples for strongly correlated phenomena are:

\section{- Unconventional superconductivity}

Unconventional superconductivity as observed in the copper oxides [BM86, Leg06], heavy fermion systems [Ste84, SS10] and iron pnictides [CEE08] is arguably the most prominent phenomena of strongly correlated electrons and the driving force in the field.

\section{- Anti- and ferromagnetism}

Though it is one of the oldest known phenomena, anti- and ferromagnetism denotes still a challenge for theoretical physicists as its complete explanation has to include strongly correlated electron effects [Blu01]. However, today the focus has shifted towards the interplay between magnetism and superconductivity and the search for new quantum phases, e.g. spin liquids [And73, Bal10, KHFP11].

\section{- Kondo effect}

The Kondo effect [Kon64, Hew93] - the increase of the resistance in diluted metals with magnetic impurities - is one of the most studied phenomena due to its very basic theoretical modelling of just one single interacting site. However, it provides a basic understanding of strong correlations and gives an access to the understanding of heavy fermion systems [Ste84].

\section{- Mott transition}

Most insulators can be explained by band structure calculations. However, for a certain class of insulators, the transition from a metal to an insulator is explicitly rooted in strong correlation effects [Mot49, IFT98].

In this work the focus is on two of these strongly correlated phenomena: The Kondo effect and anti-ferromagnetism. In order to understand the underlying mechanisms, it is advisable to understand at first which materials show strong electron correlations: For most solid state systems the long-ranged Coulomb potential proportional to $1 / r$ between electrons does not have to be explicitly taken into account as the effect of charge screening leads to an effective short range potential for the electrons [KM96]. ${ }^{3}$ For these systems band structure calculations via e.g. linear combination of atomic orbitals (LCAO), Hartree-Fock or density functional theory (DFT) are sufficient [AM76]. Most of these methods take the electron-electron interactions not at all or via an effective single particle potential into account. However, if electrons are localized in a very small region, charge screening is not effective and interaction energies dominate the physics [Mah00]. Thus, physics of strong correlations are expected in systems where the electrons are highly localized. Generally two major forms of localization can be identified: Localization due to molecular orbital theory and localization due to decreased dimensionality [Col12].

\footnotetext{
${ }^{3}$ In Thomas-Fermi theory this is the Yukawa potential $e^{-\alpha r} / r$.
} 


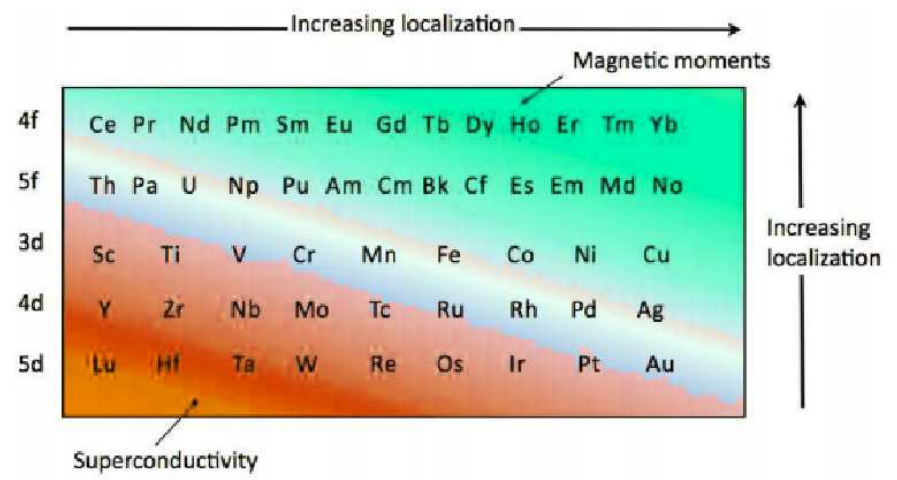

Figure 1.1: Figure adapted from Ref. [Col12]. The Kmetko-Smith diagram is shown. The trend for increasing localization with filling can be seen within the transition metals and lanthanides/actinides.

From molecular orbital theory it is known that electrons are strongest localized in the $d$ and $f$ orbitals [MM03]. Furthermore, for magnetic interactions the $d$ or $f$-shells have to be just partially filled to provide a net magnetic moment due to Hund's rules [AM76]. This is for example the case for the $3 d$ transition metals, e.g. manganese $(\mathrm{Mn})$, iron $(\mathrm{Fe})$, cobalt (Co) and nickel (Ni). These have the atomic configuration $[A r] 3 d^{x} 4 s^{2}, x=5,6,7,8$ [MM03]. In Fig. 1.1 the Kmetko-Smith diagram is shown [SK83, Col12]. The transition metals (lower three lines $3 d, 4 d, 5 d$ ) with single to fully filled $d$-shells and the lanthanoide and actinoide (upper two lines $4 f, 5 f$ ) with partially filled $f$-shells are displayed. The color encodes the trend for increasing localization of the electrons in these shells, which can lead to the described strong correlation phenomena. For example, it is known that strong correlations within the narrow $d$-bands of iron lead to itinerant ferromagnetism [Blu01].

Another possibility to have highly localized electrons are systems in low dimensions. An example are the famous cuprate superconductors, e.g. $\mathrm{Bi}_{2} \mathrm{Sr}_{2} \mathrm{Ca}_{n-1} \mathrm{Cu}_{n} \mathrm{O}_{2 n+4+x}$, that consist of weakly coupled two-dimensional copper oxide planes [BM86, Leg06]. In this reduced dimensionality localization is enhanced and plays an important role in the observed unconventional superconductivity. Other compounds like the corner-sharing chain cuprates $\mathrm{Sr}_{2} \mathrm{CuO} \mathrm{O}_{2}$ or $\mathrm{SrCuO}_{3}$ show even (quasi) one-dimensional behaviour. They are realizations of one-dimensional Mott insulators and show the famous spin-charge separation $\left[\mathrm{KMS}^{+}\right.$96, $\mathrm{KHB}^{+} 04, \mathrm{KKR}^{+}$06, $\mathrm{JFG}^{+}$09, ST10]. Single magnetic impurities in metals are often considered as zero-dimensional systems as the strongly localized $d(f)$-shell of the impurity can be regarded as an effective interacting site in a bath of non-interacting electrons. Another example for zero-dimensional systems are artificially produced quantum dots [GGSM+98, Sha08]. Of course all of these system are still three dimensional, however the relevant physics is confined in a subspace of lower dimension. 


\section{\begin{tabular}{l|l} 
& \\
Spectral functions & 1.2
\end{tabular}}

The listed phenomena are traceable by the measurement of physical observables. In particular, for metals conductivity measurements at different temperatures are standard, but also other thermodynamic quantities like specific heat have been intensively measured. Due to the invention of the scanning tunnelling microscope by Binning and Rohrer in 1987 [BR87] and developments in angle-resolved photo emission spectroscopy [Hüf03] as well as neutron scattering [Shu95] spectral functions are today accessible in experiments with high resolution.

Most of the described phenomena just occur below a critical temperature $T_{c}$ and can be best analysed without thermodynamic fluctuations close to $T=0$. The system is then in its ground state. Therefore in this work all spectral functions are calculated at $T=0$. Excitations from the ground state are a way to probe materials that show the discussed phenomena. Let me introduce the concepts by discussing a specific example: For electronic systems an excitation can for example be created by adding an electron at a certain position $x$ at time $t=0$ and removing it at the same position at a later time $t^{\prime}>0$. This kind of excitation is usually not an eigenstate of the system, but can be expressed by a superposition of many eigenstates $\left|E_{n}\right\rangle$ with energy $E_{n}:{ }^{4}$

$$
|\Psi(x, t=0)\rangle=\sum_{n} c_{n}\left|E_{n}\right\rangle
$$

The excitation will evolve in time, whereby the modes are given by the eigenenergies of the system:

$$
\left|\Psi\left(x, t^{\prime}\right)\right\rangle=e^{-i H t^{\prime}}|\Psi(x, 0)\rangle=\sum_{n} c_{n} e^{-i E_{n} t^{\prime}}\left|E_{n}\right\rangle .
$$

The (spectral) decomposition of the excitation into the eigenenergies (modes) of the system is given by the spectral function. Precisely, the spectral function is a function of the energy with peaks at the eigenenergies of the system weighted with the decomposition coefficients $c_{n}$ of the excitation: ${ }^{5}$

$$
\rho(\omega>0)=\sum_{n}\left|c_{n}\right|^{2} \delta\left(\omega-E_{n}^{\prime}\right) .
$$

Thus, the special feature of the spectral function is that it does not only provide information about the excited state of the system, but it provides information about the eigenenergies of the system. Therefore by a single measurement of the spectral function much more information about the system can be gathered. However, its theoretical calculation is also usually much more involved than the calculation of e.g. static quantities that just provide information about a single state.

\footnotetext{
${ }^{4}$ This vivid explanation is just valid for a special kind of spectral function. The spectral function is generally introduced in Sec. 2.3.2.

${ }^{5}$ Precisely, the eigenergies are given via the difference to the ground state energy $E_{n}^{\prime}=E_{n}-E_{0}$. For a continuous system the spectral function will also be continuous.
} 


\section{\begin{tabular}{l|l} 
Methods & 1.3
\end{tabular}}

The calculation of spectral functions for strongly correlated electron systems is difficult due to the many information provided by this quantity. The strong correlations eventually forbid a treatment via standard band structure calculation tools, e.g. density-functional theory (DFT) [JG89] or Green's function methods [AM76]. The exact full solution of the many-particle Schrödinger equation, that describes the full condensed matter system involving all electrons with all interactions, will be forever impossible due to the huge number of particles of the order of $10^{23}$ atoms. Thus effective models that just concentrate on the essential physics with a much smaller number of degrees of freedom provide an access to these phenomena. Albeit the use of effective Hamiltonians that dramatically decrease the number of involved particles and interactions, analytical tools are in most cases limited to one dimension or to specific sets of parameters. Therefore, in most cases, one has to resort to numerical techniques. However, the exponential scaling of the Hilbert space makes a complete solution via numerical exact diagonalization, e.g. the Householder or Lanczos algorithm [BDD ${ }^{+} 00$, GvL96], in the most cases impossible. Nevertheless, results for just a few particles and finite-size scaling can give hints for the behaviour of larger systems.

Arguably, the most successful theory for interacting systems is Landau's Fermi liquid theory [Lan58] which can describe metals even with large interactions between the particles in the normal state i.e. in the absence of any symmetry breaking. There, a one-to-one correspondence between the energy levels of some non-interacting system and the interacting system under consideration exists and all thermodynamic and spectral quantities are therefore given by the non-interacting ones with suitable rescaled effective parameters [Sch95]. Even after a phase transition to a symmetry broken phase systems can sometimes still be described by Fermi liquid theory below the critical temperature $T_{C}$. However, in some of the mentioned interesting phenomena the systems change to a non-Fermi liquid phase. This is the case for, e.g. the Mott-transition [IFT98]. In the studied Kondo effect the system changes from a Fermi liquid regime to a local moment regime and then to the so-called strongly coupling regime which can again be described by a Fermi liquid. ${ }^{6}$ In the characterization of these phases and phase transitions usually many scales (temperature, energy,...) are involved, which turns out to be a major challenge for analytical and numerical methods.

In the last 60 years many different algorithm and methods have been developed to calculate and approximate physical observable from effective Hamiltonians. The renormalization group (RG) [Wil83, Gol92] turned out to be a big breakthrough in order to calculate physical properties on different scales. In particular, it allows to investigate the different phases and phase transitions. The main idea is to provide a mapping between Hamiltonians which iteratively applied (in the language of the RG: "under the RG flow") can end up in a fixed-point. A further breakthrough was Wilson's numerical renormalization group (NRG) [Wil75, BCP08] that explicitly allows to solve numerically the

\footnotetext{
${ }^{6}$ In the Kondo effect these transitions are no phase transitions as no symmetry breaking is involved. Therefore these transitions are named cross-overs and the name regime is used instead of phase.
} 
Hamiltonian for quantum impurity models at every iteration of the RG flow. In the NRG every iteration can be related to a temperature and therefore the system can be solved on a large temperature respectively energy scale. Closely related to the renormalization group is the idea of universality and universality classes [Gol92, Col12]. The origin of this latter concept is that terms in the Hamiltonian may vanish under the RG transformation. Therefore one can construct a class of Hamiltonians which have the same fixed-point structure under the RG flow and thus show the same physics - they show universal behaviour and lie in the same universality class. This proof of universality in the end also justifies a particular choice of effective models in the beginning. In this work the NRG is applied for the calculation of spectral functions that show the Kondo effect.

Inspired by the success of the renormalization group Steve White formulated a real space renormalization group called density matrix renormalization group (DMRG) [Whi92, Whi93, Sch11] which turned out to be very successful for one-dimensional systems. Later it was shown that the DMRG is actually a variational wavefunction method and not a renormalization group in the original sense. The (ground) state of the one-dimensional quantum system is given via a variational wavefunction called matrix product states (MPS) [FNW92, OlR95]. This ansatz is very precise even for systems of a few hundred sites so that emergent phenomena, like the famous spin-charge separation in models of onedimensional interacting fermions, are observable [KSZ05]. For higher dimensional systems variational states have been formulated in a similar way called tensor network states. The most popular ones are projected entangled pair states (PEPS) [VC04, MVC07] and the multiscale entanglement renormalization ansatz (MERA) [Vid07, Vid08]. However their full potential is still unexamined. In this work an extension for the DMRG/MPS is developed to calculate spectral functions for one-dimensional anti-ferromagnetic spin chains.

Up to now also many other variations of the renormalization group idea have been developed in order to calculate fermionic systems. For example flow equations for Hamiltonians based on infinitesimal unitary transformations have been developed by Wegner [Weg94] and used by Kehrein [Keh06] mainly for quantum impurity models. Functional renormalization group was another development to tackle fermionic systems up to twodimensional systems, see Ref. [MSH ${ }^{+}$12] for an overview. Quantum Monte Carlo methods give a non-deterministic access to quantum many-body problems [LB05]. There are various successful algorithms for fermionic and bosonic systems, however most of them work at finite temperatures. For fermionic models they further have the (in)famous 'sign problem'. The development of the dynamic mean field theory (DMFT) [KV04] denotes another route to the solution of (translationally invariant) many-body problem. Here the lattice is mapped iteratively to an impurity problem which is solved self-consistently for the Green's function. The DMFT is actually just one algorithm in the class of self-energy functional theory [Pot12], which further includes, e.g. the variational cluster approximation.

To summarize, in the last 60 years many different algorithms to tackle the quantum many-body problem have been developed from which just the most important ones - in the author's view - could be listed. However, no single algorithm has been dominating the field. For every problem one has to find the optimal algorithm or one has to apply several algorithms to find the solution from different directions. The final two sections 
of the introduction give a short description of the two subjects of this thesis and give a reasoning for the chosen methods.

\section{\begin{tabular}{l|l} 
Long range Kondo signatures & 1.4
\end{tabular}}

In 1908 H. K. Onnes developed the liquefaction of helium that made it possible to cool down systems to a few Kelvin. Three years later this technique helped him to be the first to discover superconductivity by studying the resistivity of mercury [Onn11], that vanishes below a critical temperature $T_{C}$. Ordinary metals like copper in comparison show a monotonous decrease of the resistance with temperature proportional to $T^{5}$ - Bloch $T^{5}$ law - through phononic contributions at low temperatures to a finite resistance at $T=0$, see Ref. [AM76] for an introduction. In a simple picture the conduction electrons are only hindered in their motion by lattice vibrations that freeze out when the temperature is lowered. At $T=0$ only defects in the crystal and impurity scattering will contribute to a finite resistivity. In the 1930s Hildebrand et al. [Hil37] discovered an increase in the resistivity with decreasing temperature for metals with added single magnetic atoms - impurities with unoccupied $d$ - or $f$-shells. This leads to a minimum in the resistivity around a specific temperature for these materials - later on called the Kondo effect.

These two unusual effects, superconductivity and the Kondo effect, stayed a theoretical

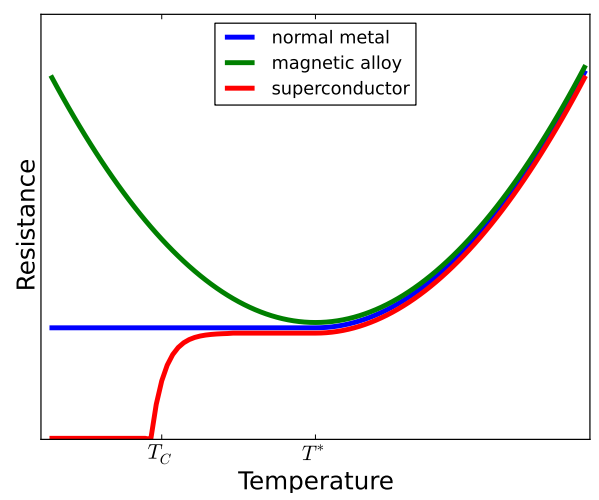

Figure 1.2: The schematic behaviour of the resistance at low temperatures for an ordinary metal (blue), a dilute magnetic alloy showing the Kondo effect with resistance minimum at $T^{*}$ (green) and a superconducting metal with critical temperature $T_{C}$ (red).

puzzle for a long time. A phenomenological theory for conventional superconductivity was found by Landau and Ginzburg [GL50] in the beginning of the 1950s and it took only seven more years until Bardeen, Cooper and Schrieffer found a complete microscopic theory for conventional superconductivity in 1957 [BCS57a, BCS57b]. In 1964 Jun Kondo found a first explanation for the increase of the resistivity for metals with additional magnetic impurities - therefore it was named after him [Kon64]. A full comprehensive solution was given later on by Wilson with the development of the numerical renormalization group [Wil75].

The Kondo effect is characterized by the formation of a strongly correlated many body state between the electrons of the partially filled $d$-orbitals of the magnetic impurities and the conduction band electrons of the host metal [Hew93]. This state emerges below a critical temperature called Kondo temperature $T_{k}$. Macroscopically it will give raise to the 
mentioned increase in the resistivity below a temperature $T^{*}=f\left(n, T_{K}\right)$ which is a function of the density of the impurities $n$ and the Kondo temperature. After many years of theoretical and experimental work the interaction between a single magnetic impurity and the surrounding conduction band electrons is mainly understood [Hew93]. However, the experimental verification of the correlations between impurity and conduction band electrons in real space is still an open issue [KG01, Aff09]. More precisely, the spatial extend of the conduction band electrons correlated with the impurity - the so-called Kondo cloud - has not been measured so far.

\footnotetext{
"After many years of theoretical and theoretical investigations, the Kondo screening cloud, as predicted by the basic Kondo model, remains undetected experimentally. This is likely due to a combination of experimental difficulties, and limitations of the basic Kondo model. It is to be hoped that further experimental and theoretical progress will eventually bring this long search to a happy conclusion."
}

(Review article: "The Kondo screening cloud: what it is and how to observe it", Affleck, 2009, [Aff09])

In this work simulations have been performed for scanning tunnelling spectroscopy (STS) measurements of a Kondo system consisting of Fe and Co impurities buried in $\mathrm{Cu}$. The STS measurements provide the local density of states (LDOS) at the surface of the copper crystal. The local density of states is a spectral function that provides information about the decomposition of a local excitation of the system. This local excitation is created by adding an electron at the surface at position $x$ to the system. In the measured LDOS clear signatures of the buried impurities can be observed. Information about their depth in mono-layers from the surface can be extracted. Therefore this measurement is a candidate for the experimental verification for the measurement of a long range Kondo signature.

The simulation of the system was done with a combination of band structure calculations via LCAO and the NRG. The applied effective Hamiltonian, the single impurity Anderson model (SIAM), was proposed by Anderson already in 1961 [And61]. From earlier work it is known that the correlated state, characterizing the Kondo effect, is visible as a sharp resonance at the Fermi energy $\epsilon_{F}$ in the spectral function of the impurity [Hew93]. The NRG is known to provide spectral functions with high resolution for this model [BCP08]. In particular, the Kondo resonance at the Fermi energy is given with high precision and therefore the NRG is the method of choice. The special focus in this project is set on the comparison of the calculated LDOS to the experimental data to underline the existence of a long range Kondo signature. Therefore, one important aspect is how the famous resonance in the spectral function of the impurity transforms into the LDOS at the surface of the sample.

The simulation and experimental data show very good agreement and hence do support the measurement of a long range Kondo signature.

\section{Dynamical spin structure factor $\mid 1.5$}

The second phenomena studied in this work, anti-ferromagnetism in a one-dimensional spin chain, was experimentally verified, e.g. in compound systems of the copper oxides. 
In those systems local moments are formed by the $\mathrm{Cu}^{2+}$ sites. One prominent example is e.g. azurite $\mathrm{Cu}_{3}\left(\mathrm{CO}_{3}\right) 2(\mathrm{OH}) 2\left[\mathrm{JOK}^{+} 11\right]$. However, these systems are in most cases not strictly one-dimensional and additional terms have to be added to the Hamiltonian. Nevertheless, a first explanation for their magnetic properties can be given with the one-dimensional spin- $\frac{1}{2}$-Heisenberg model. In this effective model the electrons are assumed to be very localized forming effective local moments (spins) which are aligned in a one-dimensional chain. The spins interact with the neighbouring spins via an exchange coupling $J$ which can be either ferromagnetic (favouring parallel aligned spins) or antiferromagnetic (favouring anti-parallel aligned spins).

The spectral function of interest for these systems is the dynamic spin structure factor which can be measured with neutron scattering. The related excitation of this spectral function is a local spin flip at a certain site. The one-dimensional Heisenberg model can be solved analytically with the Bethe ansatz [Bet31, KMB $\left.{ }^{+} 97, \mathrm{KM} 98, \mathrm{KHM} 98\right]$. The solution provides all excitation energies, however the calculation of the complete dynamic spin structure factor is non-trivial. The eigenvectors for the anti-ferromagnetic chain are called $n$-spinon excitations, whereby $n$ denotes the number of flipped spins of the superposed states in the eigenstate. From the analytic solution with the Bethe ansatz it is known that the spin structure factor is dominated by the 2-spinon excitations and just a small fraction comes from the 4-spinon excitations $\left[\mathrm{KMB}^{+} 97, \mathrm{CH} 06\right]$. Higher spinon excitations provide just a negligible contribution.

The DMRG/MPS turned out to be the most successful numerical method for onedimensional systems [Whi92, Whi93, Sch11]. A lot of effort has already been put into the calculation of spectral functions with various extensions to the original DMRG/MPS, but all show certain limitations. Therefore, in this work a new method for the DMRG/MPS is developed to calculate spectral functions for generic one-dimensional systems, even those that are not solvable with the Bethe ansatz. The newly developed method implements the Lanczos algorithm directly in MPS - the class of variational states. As a test case, it is applied to the calculation of the dynamic spin structure factor of the anti-ferromagnetic spin- $\frac{1}{2}$-Heisenberg chain. It is shown that this new algorithm can provide the 2 -spinon excitations relevant for the dynamic spin structure factor with very good precision for chains up to $\approx 70$ sites. With an applied rescaling scheme for the 2 -spinon excitations even a very good agreement with results from the Bethe ansatz in the thermodynamic limit can be obtained. 


\section{Outline}

Chapter 2: Many-body quantum physics

A short description of many-body quantum physics is presented with a special focus on the definition of spectral functions.

\section{Chapter 3: Computational tools}

The applied computational tools for the calculation of spectral functions are introduced. Three different methods are presented: (i) Exact diagonalization tools including the Lanczos algorithm for dynamic correlation functions, (ii) the renormalization group with a focus on Wilson's NRG and (iii) the DMRG in the formulation of matrix product states. The newly developed MPS Lanczos method for the calculation of spectral functions is explained in detail.

\section{Chapter 4: Long range Kondo signatures}

The theoretical description of the Kondo effect and the related single impurity Anderson model is presented. The current status of the experimental observation of the Kondo cloud and the new experimental measurements are briefly explained. The main emphasis of this chapter is on the simulation of the data by a combination of band structure and NRG calculations. In the end the results are critically reviewed and the effective model is discussed.

Chapter 5: Dynamic spin structure factor of the one-dimensional Heisenberg model

The newly developed MPS Lanczos method is applied to the dynamic spin structure factor of the spin- $\frac{1}{2}$-Heisenberg chain. The performance of the new method is critically reviewed and compared to other methods.

\section{Chapter 6: Conclusion}

The final conclusion is drawn separately for the two topics of this thesis. Prospectives for new research paths in these topics are given. 


\section{Many-body quantum physics 2}

"It would indeed be remarkable if nature fortified herself against further advances in knowledge behind the analytical difficulties of the many-body problem."

(Max Born, 1960)

From the formulation in the beginning of the last century up to now, quantum mechanics turned out to be one of the most successful theories in physics. In 1926 Schrödinger [Sch26a, Sch26b] formulated the non-relativistic quantum many-body problem for $N$ particles interacting pairwise by two-body interaction terms that vanish at infinite distance. The calculation of dynamic correlation functions for these systems is the central part of this thesis. This chapter is not a full comprehensive lecture on many-body quantum physics, but it is more an attempt to introduce the main concepts that are necessary for the further understanding of this thesis. A special focus is on the definition of dynamic correlation functions. For further details the reader is referred to the standard textbooks, e.g. Refs. [Mah00, Bru04, Nol05].

In Sec. 2.1 the relevant Hilbert space is defined and its problematic exponential scaling is discussed. Static correlation functions and expectation values are introduced in the following Sec. 2.2. In Sec. 2.3 a definition for dynamic correlation functions is given and their basic properties are discussed. At this point the special focus is on Green's functions and the relation to spectral functions.

\section{\begin{tabular}{l|l} 
Physical states and Hilbert space & 2.1
\end{tabular}}

In quantum mechanics a physical state is described by a vector $|\Psi\rangle$ that is an element of a Hilbert space $\mathbb{H}$. In this thesis, it will always be assumed that the Hilbert space is of finite dimension. This allows to avoid all the difficulties that arise with infinite dimensions, e.g. orthogonality problems and normalization. Actually, all the systems that are examined in this work can (in the end) be described by degrees of freedom that live on vertices (sites) of a finite lattice. This lattice is a discrete finite subset $\mathcal{N} \subset \mathbb{R}^{N}$ where the sites are labelled with index $l \in \mathcal{N}$. Here, $N$ denotes the space dimension of the real space. Let $\mathbb{H}_{l}$ be the local Hilbert space at one site $l \in \mathcal{N}$. Good examples are spin chains where on each site the local Hilbert space is spanned by a finite number of spin states, e.g. $\mathbb{H}_{l}^{s=\frac{1}{2}}=\operatorname{span}\{|\uparrow\rangle,|\downarrow\rangle\}$ or electrons on a lattice with maximum double occupancy 
per site, e.g. $\mathbb{H}_{l}^{e}=\operatorname{span}\{|0\rangle,|\uparrow\rangle,|\downarrow\rangle,|\uparrow, \downarrow\rangle\}$. The total Hilbert space of the lattice is then given by

$$
\mathbb{H}_{\mathcal{N}}=\otimes_{l \in \mathcal{N}} \mathbb{H}_{l}
$$

Let $d$ denote the dimension of the local Hilbert space $d=\operatorname{dim}\left(\mathbb{H}_{l}\right)$, then the dimension of the total Hilbert space results to be

$$
\operatorname{dim}\left(\mathbb{H}_{\mathcal{N}}\right)=d^{N} .
$$

This exponential scaling of the many-particle Hilbert space with the number of lattice sites $N$ is the major challenge for computational simulations.

\section{Expectation values and static correlation functions

From an experimental point of view the fundamental interest is not in the states of the Hilbert space, but in the measurement of physical observables $A$. Due to the probabilistic nature of the theory, the result of a measurement is given by an expectation value. The physical observable is described by a corresponding self-adjoint linear operator $A \in \mathbb{L}(\mathbb{H})$ and the expectation value can then be calculated by

$$
\langle A\rangle=\langle\Psi|A| \Psi\rangle \text {. }
$$

The extension to static correlation functions is straight-forward:

$$
C_{A B}=\langle A B\rangle=\langle\Psi|A B| \Psi\rangle .
$$

In order to represent a physical observable the product of $A$ and $B$ has to be a selfadjoint linear operator $A B \in \mathbb{L}(\mathbb{H})$. However, in the following these functions are also called correlation functions if the product is not a self-adjoint linear operator. A more general description, that also allows mixed states, is expressed by the density matrix $\rho$. For a pure state $|\Psi\rangle \in \mathbb{H}$ the density matrix is given by $\rho=|\Psi\rangle\langle\Psi| \in \mathbb{L}(\mathbb{H})$. The density matrix for a mixed state is given by a superposition of density matrices of pure states $\left|\Psi_{i}\right\rangle$ with a probability $p_{i}$. The density matrix is then defined by the linear operator $\hat{\rho}=\sum_{i} p_{i}\left|\Psi_{i}\right\rangle\left\langle\Psi_{i}\right| \in \mathbb{L}(\mathbb{H})$. With the density matrix the expectation value of any operator at $T=0$ can be expressed as

$$
\langle A\rangle=\operatorname{Tr}(\rho A) \quad \stackrel{\text { pure state }}{\longrightarrow} \quad\langle\Psi|A| \Psi\rangle .
$$

For finite temperatures the expectation value in a canonical ensemble is given via the partition function $Z=\operatorname{Tr} e^{-\beta \mathcal{H}}$ :

$$
C_{A B, T \neq 0}=\langle A B\rangle_{T \neq 0}=\frac{1}{Z} \operatorname{Tr}\left(e^{-\beta H} A B\right) .
$$

The physical system is described by the corresponding Hamiltonian $H \in \mathbb{L}(\mathbb{H})$. The energy of a physical system in state $|\Psi\rangle \in \mathbb{H}$ is given by the expectation value of the Hamiltonian

$$
E=\frac{\langle\Psi|H| \Psi\rangle}{\langle\Psi \mid \Psi\rangle} .
$$


At this point the expectation value is normalized to provide comparable energies. At zero temperature the system is in the ground state $\left|\Psi_{0}\right\rangle=\left|E_{0}\right\rangle$ - the state with minimal energy $E_{0}$. Therefore, at zero temperature all expectation values and correlation functions are calculated with respect to this state. ${ }^{1}$ The focus of this work is not on the ground state, but on spectral functions. For these in particular the whole spectrum of the Hamiltonian has to be calculated (see Sec. 2.3). That means, one is interested in all the eigenvectors and eigenenergies of the Hamiltonian, i.e. the complete solution of the Schrödinger equation

$$
H\left|E_{i}\right\rangle=E_{i}\left|E_{i}\right\rangle
$$

The exponential scaling of the Hilbert space with system size usually makes a complete diagonalization of the Hamiltonian impossible. Therefore, methods to get approximations for the eigenvectors of the Hamiltonian are discussed in chapter 3.

\section{Dynamic correlation functions $\mid 2.3$}

\section{Definition and Green's function $\quad 2.3 .1$}

The main focus of this work is on dynamic quantities at $T=0$. Therefore one has to extend the definition of static correlation functions, see Eqn. (2.4), to dynamic correlation functions $C_{A B}\left(t^{\prime}, t\right)$ of the type

$$
C_{A B}\left(t^{\prime}, t\right)=\left\langle A\left(t^{\prime}\right) B(t)\right\rangle \Theta\left(t^{\prime}-t\right)=\left\langle E_{0}\left|A\left(t^{\prime}\right) B(t)\right| E_{0}\right\rangle \Theta\left(t^{\prime}-t\right),
$$

where $\left|E_{0}\right\rangle$ denotes the ground state and $t^{\prime} \geq t$ for causality. In this work only timeindependent Hamiltonians $H$ are considered. Thus, it is more convenient to transform to frequency space by a Laplace transform

$$
C_{A B}(z=\omega+\mathrm{i} \eta)=\int_{0}^{\infty} C_{A B}\left(t^{\prime}, t\right) e^{i z\left(t^{\prime}-t\right)} d\left(t^{\prime}-t\right)
$$

where $\eta$ is an infinitesimal positive number introduced for convergence. The retarded Green's function is a sum of two correlation functions that is defined via the commutator $(s=-1)$ for bosonic systems and the anti-commutator for fermionic systems $(s=1):^{2}$

$$
\begin{aligned}
G_{A, B}\left(t^{\prime}, t\right) & =\ll A\left(t^{\prime}\right), B(t) \gg_{t} \\
& =-i \Theta\left(t^{\prime}-t\right)\left\langle\left[A\left(t^{\prime}\right), B(t)\right]_{s}\right\rangle .
\end{aligned}
$$

A physical interpretation of this Green's function can be given e.g. for the example mentioned in the introduction, see Sec. 1.2. There the operators $A$ and $B$ are the annihilation and creation operators $c_{i}, c_{j}^{\dagger}$ for electrons (fermions) acting on site $i, j$ of a lattice. The Green's function is then related to the probability of removing an electron at time $t^{\prime}$ at

\footnotetext{
${ }^{1}$ Here it is assumed that the system has no ground state degeneracy.

${ }^{2}$ Precisely, $s=-1$ has to be used, if at least one operator is bosonic. If both operators are odd in fermionic creation and annihilation operators $s=1$ has to be used.
} 
site $i$ under the constraint that it was added at time $t$ at site $j$. The Green's function can be written as a sum of correlation functions:

$$
\begin{aligned}
& G_{A B}^{>}\left(t^{\prime}, t\right)=-i \Theta\left(t^{\prime}-t\right)\left\langle A\left(t^{\prime}\right) B(t)\right\rangle=-i C_{A B}\left(t^{\prime}, t\right) \\
& G_{A B}^{<}\left(t^{\prime}, t\right)=-i \Theta\left(t^{\prime}-t\right)\left\langle B(t) A\left(t^{\prime}\right)\right\rangle=-i C_{B A}\left(t, t^{\prime}\right) \\
& G_{A B}\left(t^{\prime}, t\right)=G_{A B}^{>}\left(t^{\prime}, t\right)+s G_{A B}^{<}\left(t^{\prime}, t\right)=-i\left(C_{A B}\left(t^{\prime}, t\right)+s C_{B A}\left(t, t^{\prime}\right)\right) .
\end{aligned}
$$

The correlation functions are often also named as lesser $G_{A B}^{<}$and greater $G_{A B}^{>}$Green's functions. For this work the emphasis is on the representation of the Green's function in the frequency domain, which can be obtained by a Laplace transform:

$$
\begin{aligned}
G_{A B}(\omega) & =\int_{0}^{\infty} G_{A B}\left(t, t^{\prime}\right) e^{i(\omega+i \eta)\left(t^{\prime}-t\right)} d\left(t^{\prime}-t\right) \\
& =\ll A\left(t^{\prime}\right), B(t) \gg_{\omega} \\
& =\left\langle E_{0}\left|A \frac{1}{\omega+i \eta-\left(H-E_{0}\right)} B\right| E_{0}\right\rangle+s\left\langle E_{0}\left|B \frac{1}{\omega+i \eta+\left(H-E_{0}\right)} A\right| E_{0}\right\rangle \\
& =G_{A B}^{>}(\omega)+s G_{A B}^{<}(\omega) .
\end{aligned}
$$

In the last line the Green's function is written in the resolvent expression. Useful identities to relate different Green's function can be obtained from the equation of motion:

$$
\omega \ll A, B \gg_{\omega}=\left\langle[A, B]_{s}\right\rangle+\ll[A, H]_{s}, B \gg_{\omega} .
$$

\section{Spectral functions 2.3 .2}

In a broader sense a spectral function is defined as the difference between advanced and retarded Green's function, i.e. the jump across the frequency axis. In a narrower sense the spectral function is just defined as the imaginary part of the retarded Green's function:

$$
\rho_{A B}(\omega)=-\frac{1}{\pi} \operatorname{Im} G_{A B}(\omega) .
$$

Using the Dirac identity

$$
\frac{1}{\omega-\epsilon(k)+i \eta} \rightarrow \lim _{\eta \rightarrow 0} \mathcal{P} \frac{1}{\omega-\epsilon(k)+i \eta}-i \pi \delta(\omega-\epsilon(k))
$$

one can derive the Lehmann representation for the spectral function. For $B=A^{\dagger}$ it is given by:

$$
\begin{aligned}
\rho_{A A^{\dagger}}(\omega>0) & =-\lim _{\eta \rightarrow 0} \frac{1}{\pi} \operatorname{Im}\left\langle E_{0}\left|A \frac{1}{\omega-\left(H-E_{0}\right)+\mathrm{i} \eta} A^{\dagger}\right| E_{0}\right\rangle \\
& =\sum_{n}\left|\left\langle E_{n}\left|A^{\dagger}\right| E_{0}\right\rangle\right|^{2} \delta\left(\omega-\left(E_{n}-E_{0}\right)\right) \\
\rho_{A A^{\dagger}}(\omega<0) & =s \sum_{n}\left|\left\langle E_{n}|A| E_{0}\right\rangle\right|^{2} \delta\left(\omega+\left(E_{n}-E_{0}\right)\right) .
\end{aligned}
$$


The generalization for finite temperatures is given by inserting the Boltzmann weights $e^{-\beta \mathcal{H}}$ and the partition function $Z=\operatorname{Tr} e^{-\beta \mathcal{H}}$ :

$$
\rho_{A B}(\omega)=\frac{1}{Z} \sum_{n n^{\prime}}\left\langle E_{n}|A| E_{n^{\prime}}\right\rangle\left\langle E_{n^{\prime}}|B| E_{n}\right\rangle\left(e^{-\beta E_{n}}+s e^{-\beta E_{n^{\prime}}}\right) \delta\left(\omega+E_{n}-E_{n^{\prime}}\right) .
$$

One focus of this thesis is on the spectral function related to the imaginary part of the one particle Green's function of the creation and annihilation operators $c_{a}^{\dagger}, c_{b}$ of states $a$ and $b$ :

$$
\rho_{a b}(\omega)=-\frac{1}{\pi} \operatorname{Im} G_{a b}(\omega)=-\frac{1}{\pi} \operatorname{Im} \ll c_{a}, c_{b}^{\dagger} \gg_{\omega} .
$$

A non-interacting Hamiltonian is diagonalized by the set of eigenvectors $\left|E_{k}\right\rangle .^{3}$ Thus, the Green's function with operators $c_{k}, c_{k^{\prime}}$ is also diagonal and the related spectral function is given by:

$$
\rho_{k}(\omega>0)=-\frac{1}{\pi} \operatorname{Im} G_{k}(\omega>0)=\sum_{k^{\prime}}\left|\left\langle E_{k^{\prime}}\left|c_{k}^{\dagger}\right| E_{0}\right\rangle\right|^{2} \delta\left(\omega-\left(E_{k^{\prime}}-E_{0}\right)\right)=\delta(\omega-\epsilon(k)) .
$$

The density of states (DOS) is the trace over all spectral functions:

$$
\operatorname{DOS}(\omega)=\rho(\omega)=\operatorname{Tr}\left(\rho_{a b}(\omega)\right)=\sum_{a} \rho_{a a}(\omega) .
$$

The DOS is independent of the actual representation. For the spectral function of the non-interacting Hamiltonian in Eqn. (2.19) it has the following form:

$$
\rho(\omega)=\sum_{k} \delta(\omega-\epsilon(k))
$$

It is a sum of $\delta$-peaks at the eigenenergies of the system and thus bears the name 'density of states'. The local density of states (LDOS) is actually a spectral function defined via the one-particle Green's function in real space:

$$
\operatorname{LDOS}(\omega, x)=\rho_{x}(\omega)=-\frac{1}{\pi} \operatorname{Im} G_{x x}(\omega) .
$$

For a non-interacting Hamiltonian a transformation of the (momentum) Green's function (Eqn. (2.19)) to real space yields:

$$
G_{x x}(\omega)=\lim _{\eta \rightarrow 0} \sum_{k} \frac{\Psi_{k}(x) \Psi_{k}^{*}(x)}{\omega-\epsilon(k)+i \eta} .
$$

Now by using Eqn. (2.15) the spectral function can be written as:

$$
\rho_{x}(\omega)=-\frac{1}{\pi} \operatorname{Im} G_{x x}(\omega)=\sum_{k} \Psi_{k}(x) \Psi_{k}^{*}(x) \delta(\omega-\epsilon(k)) .
$$

\footnotetext{
${ }^{3}$ The dispersion $\epsilon(k)$ is given by the eigenenergies $E_{k}$.
} 
Thus, for a non-interacting Hamiltonians the LDOS can be interpreted as the density of states at a certain position $x$ while the DOS gives an average of it (the trace over all positions). However, in general the LDOS is a spectral function for the related Green's function with real space creation and annihilation operators. For an interacting system the LDOS can be calculated via Eqn. (2.16).

$$
\rho_{x}(\omega>0)=\sum_{n}\left|\left\langle E_{n}\left|c_{x}^{\dagger}\right| E_{0}\right\rangle\right|^{2} \delta\left(\omega-\left(E_{n}-E_{0}\right)\right)
$$

where the interpretation as density of states at a certain position $x$ is subtle. Thus, for interacting systems the direct interpretation as spectral decomposition of the state $c_{x}^{\dagger}\left|E_{0}\right\rangle$ into the eigenstates of the system is preferred in this work, see discussion in the introduction - Sec. 1.2. In the context of spectral functions the eigenenergies are called spectral poles $\omega_{n}$ and the weighting factors $\Omega_{n}=\left|\left\langle E_{n}\left|c_{x}^{\dagger}\right| E_{0}\right\rangle\right|^{2}$ are called spectral weights. 


\title{
Computational tools
}

\begin{abstract}
"Typically the way physics has done is as follows: (1) formulate a theoretical model; (2) (approximately) solve the model to make a prediction; then (3) experimentally test the prediction. If the experiment confirms the prediction then declare success. If not, then go back to the drawing board. Each of these three steps typically requires considerable ingenuity, insight, and resourcefulness to carry out. However, of these three steps, the second has a somewhat algorithmic flavour: if one only threw enough computational resources at the model then surely it could be solved? This echoes Hilbert's Entscheidungsproblem: perhaps for any physical model, when sufficiently clearly formulated, there exists a procedural algorithm to extract predictions, at least up to some acceptable error margin? The analogy with the Entscheidungsproblem here has been specifically chosen to foreshadow the answer: no, there is no such general efficient method."
\end{abstract}

(Article: "Hamiltonian Complexity", Osborne, 2012,[Osb12])

The complete solution to the non-relativistic quantum many-body problem is equivalent to finding all the solutions $\Psi_{i}\left(\mathbf{x}_{1}, . . \mathbf{x}_{N}\right)$ of the Schrödinger equation. Due to the exponential scaling of the Hilbert space this problem is for most interacting systems an NP-hard problem. This eventually means that direct (exact) computational simulations are constrained to only a very few particles. Even simpler questions, e.g. what is the state with the lowest energy of a one-dimensional (gapped) system, is in the worst case still a NP-hard problem ${ }^{1}$ [Eis06]. Nevertheless, in the last 50 years many successful computational algorithms have been developed to tackle these problems and to find approximate solutions. In this work, dynamic correlation functions are calculated with the NRG for zero-dimensional systems (one interacting state coupled to a bath of non-interacting states) and with the DMRG/MPS for one-dimensional systems.

In Sec. 3.1 exact diagonalization techniques are presented with a focus on the limitations of these methods. Then in Sec. 3.2 the renormalization group is described. It starts with a note on the history of the RG in Sec. 3.2.1, followed by a mathematical definition of the RG transformation in Sec. 3.2.2. In the next Sec. 3.2.3 Wilson's NRG is explained in detail. In Sec. 3.3 an introduction to MPS is given starting with the general formalism in Sec. 3.3.1 to Sec. 3.3.5, closing with some notes on the differences between DMRG and MPS in Sec. 3.3.6. A special focus is on the calculation of spectral functions in Sec. 3.3.7 with the two newly developed Lanczos methods.

The content of parts of Sec. 3.3.7 is published as a Rapid Communication in Physical Review $\mathrm{B}\left[\mathrm{DHP}^{+} 11\right]$ and as a regular article in Physical Review B [DWH $\left.{ }^{+} 12\right]$. The first work was a collaboration with Andreas Honecker, Robert Peters, Reinhard Noack and Thomas Pruschke. The second work was a collaboration with Andreas Honecker, Anton Wöllert, Ian P. McCulloch, Ulrich Schollwöck and Thomas Pruschke. It may be that

\footnotetext{
1 "NP" stands for non-deterministic polynomial time" in computational complexity theory and describes roughly speaking problems that cannot be solved by any algorithm in polynomial time (time that scales polynomial in system size, length, ...).
} 
some parts of the following chapter resemble the manuscript of Refs. [DHP $\left.{ }^{+} 11, \mathrm{DWH}^{+} 12\right]$. The MPS code was provided by Ian P. McCulloch and the basis of the DMRG code was provided by Robert Peters.

\section{\begin{tabular}{l|l} 
Exact diagonalization techniques & 3.1
\end{tabular}}

In this work the focus is on approximative algorithms that can handle systems far beyond the ones that are accessible with exact diagonalization techniques or the Lanczos algorithm. However, in Sec. 3.3.7 the Lanczos algorithm is implemented in MPS and therefore the classical Lanczos algorithm is introduced in this section. There exists a vast amount of literature on these two topics, however this introduction is mainly based on the books by Bai et al. $\left[\mathrm{BDD}^{+} 00\right]$ and Golub and van Loan [GvL96].

\section{Exact diagonalization $\mid 3.1 .1$}

The discrete finite dimensional Hilbert space of quantum lattice models allows to express the Hamiltonian as a matrix. In order to obtain the full set of eigenvectors and eigenenergies as needed for dynamic correlation functions, see Eqn. (2.16), an exact diagonalization of the matrix must be performed. Various different diagonalization algorithms can be found in the literature of numerical mathematics, see e.g. Ref. [GvL96] for an overview. One prominent and frequently used diagonalization algorithm is the Householder algorithm that is based on an orthogonal matrix transformations. It scales with $m^{3}$ independently on the number of empty entries, where $m$ is the matrix dimension. This scaling denotes the lower limit for exact diagonalization routines and by exploiting computational resources the full diagonalization of electronic systems are usually limited to $\approx 10$ sites and of spin systems to $\approx 20$ spins [GvL96]. ${ }^{2}$

\section{\begin{tabular}{l|l} 
Lanczos algorithm & 3.1 .2
\end{tabular}}

The Lanczos algorithm belongs to the class of iterative eigensolvers, see Ref. [BDD $\left.{ }^{+} 00\right]$ for an overview. Sometimes, it is also categorized under the term of exact diagonalization algorithms, as extremal eigenvectors like ground states can be obtained close to machine precision. However, the Lanczos algorithm does not provide the complete spectrum, but only a limited number of eigenvectors. Nonetheless, it has been successfully employed in the context of calculations of Green's functions [HHK72, GB87].

\footnotetext{
${ }^{2}$ The exact system sizes are dependent on the dimension, implemented symmetries, computational ressources, ...
} 


\section{Formalism}

Within the Lanczos algorithm one recursively generates an orthogonal basis of the Krylov space. The Krylov space is spanned by iteratively applying the Hamiltonian $H$ to a starting vector $|x\rangle$ :

$$
\mathbb{K}=\operatorname{span}\left\{|x\rangle, H|x\rangle, H^{2}|x\rangle, H^{3}|x\rangle, H^{4}|x\rangle \ldots\right\} .
$$

The orthogonality of the vectors is reached essentially by a Gram-Schmidt routine [PTVF92], which can be formulated by a recursion formula ${ }^{3}$ that generates the so-called Lanczos vectors:

$$
\begin{aligned}
\left|f_{n+1}\right\rangle & =H\left|f_{n}\right\rangle-a_{n}\left|f_{n}\right\rangle-b_{n}^{2}\left|f_{n-1}\right\rangle, \quad\left|f_{0}\right\rangle=|x\rangle \\
a_{n} & =\left\langle f_{n}|H| f_{n}\right\rangle /\left\langle f_{n} \mid f_{n}\right\rangle, \\
b_{n}^{2} & =\left\langle f_{n} \mid f_{n}\right\rangle /\left\langle f_{n-1} \mid f_{n-1}\right\rangle, \quad b_{0}=0 .
\end{aligned}
$$

The full Hamiltonian is then mapped onto this orthogonal Krylov space, resulting in a tridiagonal structure:

$$
H_{\mathrm{eff}}=\left(\begin{array}{ccccc}
a_{0} & b_{1} & 0 & \ldots & 0 \\
b_{1} & a_{1} & b_{2} & \ddots & \vdots \\
0 & b_{2} & \ddots & \ddots & 0 \\
\vdots & \ddots & \ddots & \ddots & b_{n-1} \\
0 & \ldots & 0 & b_{n-1} & a_{n-1}
\end{array}\right) .
$$

Due to the tridiagonal form, this effective Hamiltonian can be easily diagonalized. Additionally, it can be shown that the extremal eigenvalues are good approximations [BDD $\left.{ }^{+} 00\right]$ to the extremal eigenvalues of the original system. This procedure, originally developed for finding the extremal eigenvalues of large sparse matrices, can be adapted to the calculation of dynamic correlation functions [GB87]. For such functions, see Eqn. (2.16), actually not the full set of eigenvectors is of interest, but only those vectors which have non-zero overlap with the vector $A^{\dagger}\left|E_{0}\right\rangle$.

Thus, one starts the Lanczos algorithm with the vector $\left|f_{0}\right\rangle=A^{\dagger}\left|E_{0}\right\rangle / \sqrt{\left\langle E_{0}\left|A A^{\dagger}\right| E_{0}\right\rangle}$, so that those eigenvectors and eigenenergies are generated that give a non-zero contribution to the dynamic correlation function. If one diagonalizes $H_{\text {eff }}$ - obtained in this way - its eigenvectors and eigenvalues give direct access to the (lowest) poles and spectral weights of Eqn. (2.16). The Lanczos method was applied to the anti-ferromagnetic spin- $\frac{1}{2}$ Heisenberg chain (with periodic boundary conditions) by Fledderjohann et al. [FKMW95] and later by Karbach et al. $\left[\mathrm{KMB}^{+} 97\right]$ to obtain spectral weights and poles for chains up to 28 sites.

\section{Ghost problem}

The Lanczos method is obviously limited by system size, as all "exact diagonalization" methods are. However, there are more subtle issues: The Lanczos algorithm will give the

\footnotetext{
${ }^{3}$ Within the application of the method to Green's functions the recursion formula is sometimes called Lanczos-Haydock recursion formula. See Refs. [HHK72, Hay80] for further details.
} 
best convergence for the extremal eigenvalues, which are contained in the starting vector. The interior eigenvalues will only converge after a large number of Lanczos iterations. In most cases, this turns out to be impossible as numerical errors will destroy the orthogonality of the Lanczos vectors. This is the well-known "ghost problem" [BDD ${ }^{+}$00, CW85] which leads to spurious (double) eigenvalues. There are many approaches to resolve the loss of orthogonality. The two most popular ones are total or partial re-orthogonalization and restarting procedures $\left[\mathrm{BDD}^{+} 00\right]$. In a partial or total reorthogonalization procedure one tries to recover orthogonality of the current Lanczos state by removing the overlap to the previous ones explicitly after several steps or after each step:

$$
\left|\tilde{\psi}_{n}\right\rangle=\left|f_{n}\right\rangle-\sum_{i}^{n-1}\left\langle f_{n} \mid \psi_{i}\right\rangle\left|\psi_{i}\right\rangle, \quad\left|\psi_{n}\right\rangle=\frac{1}{N}\left|\tilde{\psi}_{n}\right\rangle .
$$

Here, $N=\sqrt{\left\langle\tilde{\psi}_{n} \mid \tilde{\psi}_{n}\right\rangle}$ accounts for the proper normalization. However, even with renormalization methods, "ghosts" stay a problem in the Lanczos algorithm.

\section{Stopping criteria}

Two obvious questions are, when to stop the Lanczos iteration and how to distinguish real excitation energies from ghosts. The usual criterion for the quality of an approximate pole position (eigenenergy) $\omega_{k}$ is the residual. The residual vector is defined via

$$
\left|r_{k}\right\rangle=H\left|E_{k}\right\rangle-\omega_{k}\left|E_{k}\right\rangle
$$

and the residual by

$$
r_{k}=\left\langle r_{k} \mid r_{k}\right\rangle=\left\langle E_{k}\left|\left(H-\omega_{k}\right)^{2}\right| E_{k}\right\rangle
$$

The residual gives a measure, how well the pole $\omega_{k}$ and (normalized) vector $\left|E_{k}\right\rangle$ approximate an eigenvalue and an eigenvector of $H$. In this thesis, the exact position and weight of the spectral poles are of interest and therefore a recipe to extract these quantities with the help of the residuals was developed:

1. Remove all poles with a spectral weight below $\Omega_{c u t}$ and a residual which is larger than $r_{\text {cut }}$, because they are very likely ghosts.

2. Follow the convergence of each of the remaining poles and take those with the smallest residual.

\section{Thermodynamic limit and finite-size scaling}

In the interesting thermodynamic limit dynamic correlation functions become continuous functions. Thus methods which turn the discrete finite-size data into a continuous form (approximating the data in the thermodynamic limit) are of special interest. Continued fraction expansion and the direct broadening of spectral poles are two such methods to get a continuous version of the discrete dynamic correlation functions. Using the direct 
broadening of the spectral poles, the delta peaks in the dynamic correlation function, see Eqn. (2.16), can be broadened by e.g. a Lorentzian with broadening parameter $\eta$ :

$$
\rho_{A}(\omega+i \eta)=\frac{1}{\pi} \sum_{n}\left|\left\langle E_{n}|A| E_{0}\right\rangle\right|^{2} \frac{\eta}{\left(\omega-\left(E_{n}-E_{0}\right)\right)^{2}+\eta^{2}}, \omega>0 .
$$

Equivalently, one can also calculate directly a "smooth" spectral function by using the continued fraction expansion [GB87]

$$
\rho_{A}(z)=-\frac{1}{\pi} \operatorname{Im} \frac{\left\langle E_{0}\left|A A^{\dagger}\right| E_{0}\right\rangle}{z-a_{0}-\frac{b_{1}^{2}}{z-a_{1}-\frac{b_{2}^{2}}{z-\ldots}}},
$$

with $z=E_{0}+\omega+\mathrm{i} \eta$. Both methods to obtain a "smooth" spectral function are equivalent, if one neglects special details about the termination of the continued fraction and if a Lorentzian is used for the direct broadening. For large systems and large broadenings this gives a decent approximation of the broadened correlation function in the thermodynamic limit. With the Lanczos method the manageable system sizes are rather small and therefore a finite-size scaling $L \rightarrow \infty$ with fixed broadening $\eta$ of the data has to be performed first. Extracting the $\eta \rightarrow 0$ limit a posteriori by removing the broadening by a deconvolution is well-known to be a numerically ill-defined problem. There is no unique solution and the best solution can only be given by probability arguments, see Ref. [RU05, Dar09] for details. Without a finite-size scaling one would essentially try to "deconvolute back" to a finite number of delta peaks. In summary the extrapolation to the interesting thermodynamic limit (i) $L \rightarrow \infty$, (ii) $\eta \rightarrow 0$, from broadened data is far from trivial, especially as the two limits are not to be interchanged.

\section{Finite-size scaling of single spectral weights}

However, knowing the explicit positions of the spectral poles for several finite system is advantageous, because it allows, among other things, to perform proper finite-size scaling for low-energy excitations and to clearly identify possible gaps in the spectrum. Furthermore, Holzner et al. [HWM $\left.{ }^{+} 11\right]$ suggested recently to use a systematic rescaling of the spectral weights of chains with different length to approximate the dynamic correlation function in the thermodynamic limit. The idea of this rescaling is to directly translate the definition of the spectral function reading "spectral weight per unit frequency interval" into a mathematical formula $\left[\mathrm{HWM}^{+} 11\right]$. Each of the weights $\Omega_{n}=\left|\left\langle E_{n}|A| E_{0}\right\rangle\right|^{2}$ is rescaled by the width of the energy interval

$$
\Delta_{n}=\left(\epsilon_{n+1}-\epsilon_{n-1}\right) / 2
$$

where the pole is located in. For the lower bound $\omega_{1}$ of the spectral function this definition has to be replaced by $\Delta_{1}=\left(\epsilon_{2}+\epsilon_{1}\right) / 2-\omega_{1}$. Using this rescaling scheme, all spectral weights will now lie on the spectral function calculated in the thermodynamic limit. Combining the spectral weights/poles of chains with different lengths, one can then generate a high resolution approximation to the spectral function in the thermodynamic limit and consequently obtain a controlled approach to the problematic limit $\eta \rightarrow 0, L \rightarrow \infty$. 


\title{
\begin{tabular}{l|l} 
Renormalization group & 3.2
\end{tabular}
}

\begin{abstract}
"The "renormalization-group" approach is a strategy for dealing with problems involving many length scales. The strategy is to tackle the problem in steps, one step for each length scale. In the case of critical phenomena, the problem, technically, is to carry out statistical averages over thermal fluctuations on all size scales. The renormalization-group approach is to integrate out the fluctuations in sequence, starting with fluctuations on an atomic scale and then moving to successively larger scales until fluctuations on all scales have been averaged out."

(Nobel lecture: "The renormalization group and critical phenomena", Wilson, 1983, [Wil83])
\end{abstract}

\section{\begin{tabular}{l||l} 
Introduction & 3.2 .1
\end{tabular}}

This introduction gives an overview of the main ideas and historic development of the renormalization group ( $R G$ ) and is mainly based on the seminal papers by Wilson, see Refs. [Wil75, Wil83]. The purpose is to explain the problems and approaches that led to the development of the NRG.

One of the most difficult tasks in theoretical physics is the correct description of the statistical continuum limit [Wil75]. A very prominent example are lattice models with fixed lattice spacing and local interactions. In the continuum limit one does not expect the lattice structure to be visible any more and one is mainly interested in the emergence of global properties. Critical phenomena, like phase transitions, in these systems are accompanied by long wavelength fluctuations [Wil75]. The maximum of these long wavelength fluctuations is given by the correlation length $\xi$, which diverges exactly at the critical temperature.

In the classical continuum limit one changes to the hydrodynamic picture and describes the system by a fluid with a dominant fixed wavelength with no reference to the atomic structure and this allows to introduce continuum forms for e.g. density and pressure [Wil75]. An attempt to describe the fluctuations in a critical system in a hydrodynamic picture unfortunately completely fails. The long wavelength fluctuations are not the dominant fluctuations, the fluctuations over all energy scales are crucial for the critical phenomena. The statistical continuum limit is therefore indicated by the absence of a dominant scale - neither the correlation length nor the atomic spacing are dominant.

Physicists first encountered this problem in quantum electrodynamics and particle physics. There usually an integration over short wavelength fluctuations leads to the (in)famous divergences in calculating e.g. scattering amplitudes. Calculating the Lamb shift in 1947 Bethe [Bet47] was the first to calculate a physical property from the difference of two diverging quantities [Hae11]. Based on Bethe's idea Tomonaga [Tom46], Schwinger [Sch48b, Sch48a, Sch49], Feynman [Fey49a, Fey49b, Fey50] and Dyson [Dys49a, Dys49b] developed a mathematical scheme to calculate finite values up to every order in perturbation theory [Wil75, Hae11]. ${ }^{4}$ Further main developments of these quantum field theoretical methods - introducing the name renormalization group - are due to Stückelberg

\footnotetext{
${ }^{4}$ If the theory is 'renormalizable' - which is true if roughly speaking the number of diverging diagrams is finite.
} 
[SP53] and independently to Gell-Mann and Low [GML54] as well as Callan [Cal70] and Symanzik [Sym71]. However, these developments still remained mainly computational tools applicable to Feynman diagrams [Wil75, Hae11].

It was Wilson [Wil75] who realized that the summation of the fluctuations in the previous so-called perturbative renormalization groups are treated in the same way on all energy scales. However, this only works if the expansion parameter is small leading to a small coupling of the fluctuations at different scales. It was his physical insight to realize that the "many length or energy scales are locally coupled" [Wil75]. The fluctuations on one energy scale are mainly influenced by the neighbouring energy scales - a cascade picture that should also be realized in the summation. His main idea was "to divide the full range of wavelengths into subranges of manageable proportions and consider each subrange in sequence" [Wil75].

Shortly before Wilson's breakthrough, Kadanoff [Kad66] applied a real space renormalization group method to the two-dimensional Ising model [Wil75]. Kadanoff grouped iteratively a number of spins and mapped them to a single new spin. He approximated the Hamiltonian under this transformation again by the same Hamiltonian but with renormalized coupling constants. This approach was not successful to explain the behaviour of all critical exponents, but it gave Wilson the essential idea to interpret the RG as a flow in the space of all possible Hamiltonians, that will be explained in the next section.

\section{\begin{tabular}{l|l} 
Renormalization group transformation & 3.2 .2
\end{tabular}}

This quantitative introduction to the RG is based on the formulation of Hewson [Hew93]. The purpose is to explain the renormalization flow in a more general framework that also appears in the NRG in the form of the energy flow.

The basic ingredient of the $\mathrm{RG}$ is the mapping $R$ of a Hamiltonian $H(\mathbf{K})$ with a given set of coupling constants $\mathbf{K}=\left(K_{1}, . . K_{n}\right)$ to a Hamiltonian $H\left(\mathbf{K}^{\prime}\right)$ of the same form with a new set of coupling constants $\mathbf{K}^{\prime}=\left(K_{1}^{\prime}, . . K_{n}^{\prime}\right)$ :

$$
R(H(\mathbf{K}))=H\left(\mathbf{K}^{\prime}\right) \Longleftrightarrow R(\mathbf{K})=\mathbf{K}^{\prime}
$$

The renormalization transformation is usually specified by a continuous parameter $\alpha$ with:

$$
R_{\alpha}(\mathbf{K})=\mathbf{K}^{\prime} \quad R_{\alpha^{\prime}}\left(R_{\alpha}(\mathbf{K})\right)=R_{\alpha+\alpha^{\prime}}(\mathbf{K}) .
$$

In the last line the group character of the transformation becomes apparent. ${ }^{5}$ The most important part of the RG is the appearance of fixed points:

$$
R_{\alpha}\left(\mathbf{K}^{*}\right)=\mathbf{K}^{*}
$$

If it can be shown that the flow of a Hamiltonian (respectively the coupling constants) under the RG transformation ends in a fixed point, the physics of the system described by

\footnotetext{
${ }^{5}$ Usually the transformation does not provide an inverse, so that it usually constitutes a semi-group.
} 

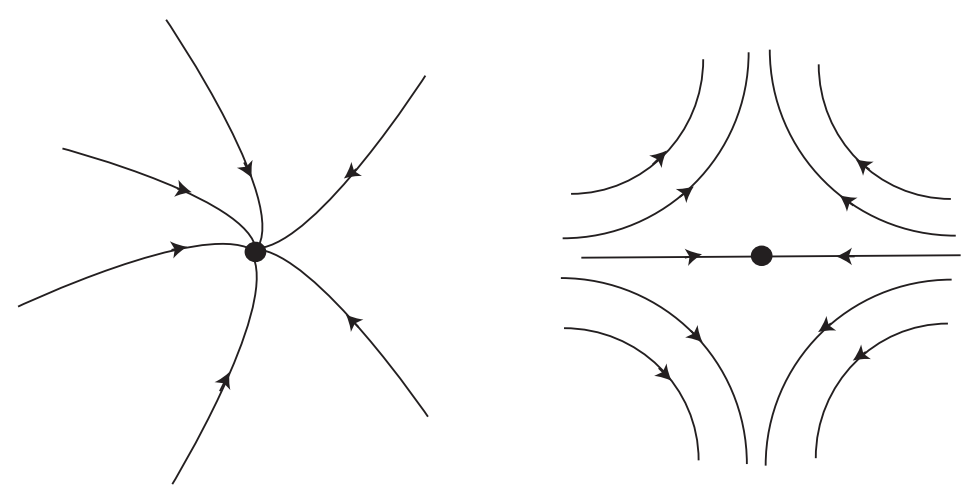

Figure 3.1: The RG flow close to fixed points. The left hand side shows a stable fixed point, the right hand side an unstable fixed point, see also Ref. [Hew93].

the Hamiltonian can be deduced from this fixed point [Gol92, Hew93]. Critical fixed points describe the singular critical behaviour - the behaviour at phase transitions - whereas trivial fixed points describe the bulk phases of the system. Therefore, the location and nature of the fixed points provide the phase diagram while the behaviour close to a critical fixed point will give critical exponents of the phase transitions. For the analysis of the RG flow close to a fixed point, one usually linearises the transformation.

$$
R_{\alpha}\left(\mathbf{K}^{*}+\delta \mathbf{K}\right)=\mathbf{K}^{*}+\mathbf{L}_{\alpha}^{*} \delta \mathbf{K}+O\left(\delta \mathbf{K}^{2}\right) .
$$

The eigenvalues $\lambda_{n}^{*}$ of the linear transformation $\mathbf{L}_{\alpha}^{*}$ are called relevant if $\lambda_{n}^{*}>1$, marginal if $\lambda_{n}^{*}=1$ and irrelevant if $\lambda_{n}^{*}<1$. Stable fixed points have only irrelevant eigenvalues, so all neighbouring trajectories end in the fixed point. Unstable fixed points have one or more relevant eigenvalues and neighbouring trajectories are eventually leading away from the fixed point. Marginal fixed points have at least one marginal eigenvalue and no relevant eigenvalues at all. For these fixed points one needs further investigations to understand the behaviour of the RG flow close to that fixed point.

\section{\begin{tabular}{l|l} 
Numerical renormalization group & 3.2 .3
\end{tabular}}

In this work the focus of the RG methods is on Wilson's numerical renormalization group (NRG) which is one special implementation of the RG. The following short description of the NRG is based on the review article of Bulla, Costi and Pruschke [BCP08]. In this work two different implementations of the NRG are used: One was provided by Robert Peters and Thomas Pruschke, the other one was provided by Rok Žitko. ${ }^{6}$

A special characteristics of the NRG is its non-perturbative nature. However, it has the drawback that the RG transformations cannot be computed analytically, but have to

${ }^{6}$ The "NRG Ljubljana" by Rok Žitko is published under GPL and can be downloaded from http:// nrgljubljana.ijs.si. In the caption of the figures the used implementation of the NRG is denoted by the abbreviations (zi) for Žitko and (pe) for Peters. 
be performed with a computer. The NRG is limited to systems consisting of an impurity which is coupled to a bath of non-interacting conduction electrons via a hybridization term:

$$
H=H_{\text {imp }}+H_{\text {bath }}+H_{\text {hyb }} .
$$

The impurity can consist of several energy levels and intra-impurity interactions, but for convenience the following description of the NRG is demonstrated using the single impurity Anderson model (SIAM) [And61] as an example:

$$
H_{\mathrm{SIAM}}=\sum_{k, \sigma} \epsilon_{k} c_{k \sigma}^{\dagger} c_{k \sigma}+\sum_{\sigma} \epsilon_{d} c_{d \sigma}^{\dagger} c_{d \sigma}+\sum_{k, \sigma}\left(V_{k} c_{d \sigma}^{\dagger} c_{k \sigma}+V_{k}^{*} c_{k \sigma}^{\dagger} c_{d \sigma}\right)+U n_{d \uparrow} n_{d \downarrow}
$$

$c_{k \sigma}^{\dagger}$ and $c_{k \sigma}$ are the usual creation and annihilation operators with spin $\sigma$ and momentum $k$ and $\epsilon(k)$ is the dispersion relation. $V_{k}$ denotes the hybridization of the impurity to the conduction band and $U$ is the interaction on the impurity level with energy $\epsilon_{d}$. A physical motivation for this model is given in Sec. 4.2. The NRG has essentially three main steps: At first the Hamiltonian is mapped onto a semi-infinite chain with exponentially decreasing hopping constants which is achieved by a logarithmic discretization of the bath. In a second step a special RG Transformation is defined for this semi-infinite chain, that has to be performed numerically including a special truncation (approximation) scheme. Finally, all interesting physical quantities are calculated.

\section{Mapping and logarithmic discretization}

The aim of this section is to map the SIAM given by Eqn. (3.15) to a semi-infinite chain model with exponentially decreasing hopping constants $t_{n}$. For a single impurity coupled to a bath, the effect of the bath to the impurity can be completely expressed by the hybridization function:

$$
\Delta(\omega)=\pi \sum_{k} V_{k}^{2} \delta\left(\omega-\epsilon_{k}\right)
$$

The geometry and characteristics of the bath like dimensionality, boundaries, surface effects are fully hidden in this function. In the following the hybridization function is assumed to be non-zero only in a finite interval $[-D, D]$ (bandwidth), which is taken to be $D=1$ for simplicity. Then the Hamiltonian can be reformulated in a form:

$$
H=H_{\mathrm{Imp}}+\sum_{\sigma} \int_{-1}^{1} d \epsilon \quad g(\epsilon) a_{\epsilon \sigma}^{\dagger} a_{\epsilon \sigma}+\sum_{\sigma} \int_{-1}^{1} d \epsilon \quad h(\epsilon)\left(d_{\sigma}^{\dagger} a_{\epsilon \sigma}+a_{\epsilon \sigma}^{\dagger} d_{\sigma}\right) .
$$

At this point, new band operators $a_{\epsilon \sigma}^{\dagger}, a_{\epsilon \sigma}$ are introduced that fulfil the standard fermionic commutation relations. The functions $g(\epsilon)$ and $h(\epsilon)$ are related to the hybridization function by:

$$
\Delta(\omega)=\pi \frac{d g^{-1}(\omega)}{d \omega} h\left[g^{-1}(\omega)\right]^{2}
$$


This relation offers some freedom to divide $\Delta(\omega)$ into the functions $g(\epsilon)$ and $h(\epsilon)$. In a next step the bath is discretized again. Here the choice for a logarithmic discretization ensures the exponentially decreasing hopping parameters. Precisely that means that one divides the energy support of the bath into sections whose width $w_{n}$ grows exponentially with energy. Therefore discretization points $x_{n}$ are introduced that fullfil:

$$
x_{n}= \pm \Lambda^{-n}, n=0,1,2, \ldots \quad \text { and } \quad w_{n}=\Lambda^{-n}\left(1-\Lambda^{-1}\right) .
$$

This logarithmic discretization incorporates Wilson's idea of a cascading picture as explained in Sec. 3.2.1. In a next step for each of these intervals a complete set of orthonormal functions is introduced: $\psi_{n p}^{ \pm}(\epsilon)$, where $p \in \mathbb{Z}$ denotes the (quasi)-momentum. The conduction operators are then expanded in this basis. To end up with a semi-infinite chain, all terms with $p \neq 0$ are dropped. This approximation is justified as all $p \neq 0$ terms couple only indirectly to the impurity. This step is followed by a Gram-Schmidt orthogonalization onto the bonding combination, for details see Ref. [BCP08]. The Hamiltonian is then given by:

$$
H_{\text {si-chain }}=H_{\text {Imp }}+\sqrt{\frac{\xi_{0}}{\pi}} \sum_{\sigma}\left(d_{\sigma}^{\dagger} c_{0 \sigma}+c_{0 \sigma}^{\dagger} d_{\sigma}\right)+\sum_{\sigma, n=0}^{\infty}\left[\epsilon_{n} c_{n \sigma}^{\dagger} c_{n \sigma}+t_{n}\left(c_{n \sigma}^{\dagger} c_{n+1 \sigma}+c_{n+1 \sigma}^{\dagger} c_{n \sigma}\right)\right] .
$$

a)

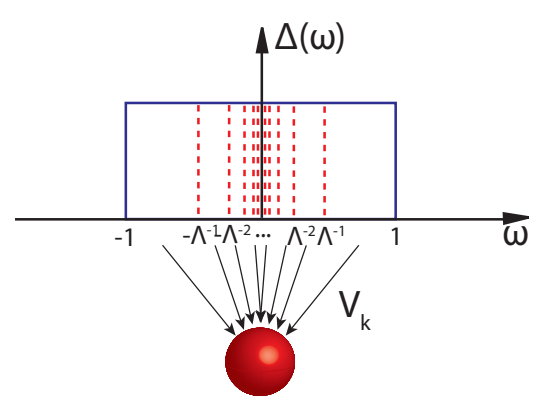

b)

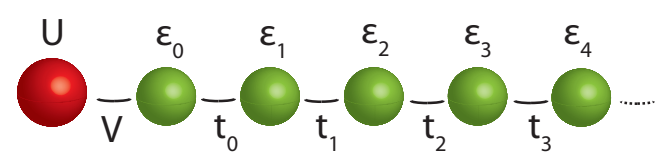

Figure 3.2: Different steps of the NRG a) The density of states of the conduction band electrons - here assumed to be constant with a bandwidth $[-1,1]$ - that couples via the hybridization $V_{k}$ to the impurity is divided into logarithmically decreasing energy sections. b) The bath of the conduction electrons is mapped on a semi-infinite chain where the first site is given by the impurity with onsite interaction $U$. Due to the logarithmic discretization the hopping constants $t_{i}$ fall off exponentially.

\section{Renormalization group transformations}

Wilson's NRG is not a classical RG in the sense that one starts with the physical Hamiltonian of interest, does the RG transformations and ends up in a fixed point Hamiltonian whose physics are known or easier to calculate and give the physics of the original Hamiltonian. In the NRG the procedure is essentially inverted. One starts with an exactly solvable Hamiltonian defined on a small Hilbert space and constructs RG Transformations that will end in the Hamiltonian which is the one of interest. The interesting RG flow is not in the Hamiltonians themselves, but in the flow of energies. In the energy flow the energy spectrum of the starting Hamiltonian describes the physics at high temperatures and under the RG flow the subsequent Hamiltonians describe the system at a 
reduced temperature. More precisely, one starts just with the impurity and hybridization term of the semi-infinite chain Hamiltonian given by Eqn. (3.20)

$$
H_{0}=\Lambda^{-1 / 2}\left(H_{\mathrm{Imp}}+\sum_{\sigma} \epsilon_{n} c_{n \sigma}^{\dagger} c_{n \sigma}+\sqrt{\frac{\xi_{0}}{\pi}} \sum_{\sigma}\left(d_{\sigma}^{\dagger} c_{0 \sigma}+c_{0 \sigma}^{\dagger} d_{\sigma}\right)\right) .
$$

The RG-Transformation ${ }^{7}$ is then given by

$$
H_{N+1}=\sqrt{\Lambda} H_{N}+\Lambda^{N / 2} \sum_{\sigma}\left[\epsilon_{N+1} c_{N+1 \sigma}^{\dagger} c_{N+1 \sigma}+t_{n}\left(c_{N \sigma}^{\dagger} c_{N+1 \sigma}+c_{N+1 \sigma}^{\dagger} c_{N \sigma}\right)\right]
$$

and will converge to the semi-infinite chain Hamiltonian given by Eqn. (3.20):

$$
H_{\text {si-chain }}=\lim _{N \rightarrow \infty} \Lambda^{-(N-1) / 2} H_{N}
$$

At this state, the above described subtle distinction has to be done: The last line has not to be understood as the fixed point of the RG Transformation. Within the NRG the relevant $\mathrm{RG}$ flow is the energy flow: the transformation of the (low-lying) energyspectrum under the $\mathrm{RG}$ transformation. In order to calculate the energy-spectrum one always numerically diagonalizes the Hamiltonian $H_{N}$ starting with $H_{0}$ :

$$
H_{N}=\sum_{m} E(m)|m\rangle\langle m|
$$

Here, $|m\rangle$ denote the eigenvectors and $E(m)$ the corresponding eigenenergies of the Hamiltonian. In the following step the Hamiltonian is set up in the product basis of the previous eigenbasis $\left|m_{N}\right\rangle$ and a new basis for the added site $|s\rangle$ :

$$
\left\langle\tilde{m}_{N+1}\left|H_{N+1}\right| \tilde{m}_{N+1}^{\prime}\right\rangle \quad \text { with } \quad\left|\tilde{m}_{N+1}\right\rangle=\left|m_{N}\right\rangle \otimes|s\rangle .
$$

Then, again, this Hamiltonian is diagonalized, a new eigenbasis $\left|m_{N+1}\right\rangle$ is set up and the next Hamiltonian is constructed. Of course, due to the exponential scaling of the Hilbert space, this iterative diagonalization cannot be done exactly after a few iterations. Therefore, the number of eigenvectors from the previous Hamiltonian, that is used to construct the basis for the next Hamiltonian, is limited to certain number $N_{s}$. One selects only the $N_{s}$ eigenvectors with the lowest eigenenergies. This truncation has to be justified:

"First of all, there is no guarantee that this scheme will work in practical applications, and its quality
should be checked for each individual application. An important criterion for the validity of this approach is
whether the neglect of high-energy states spoils the low-energy spectrum in subsequent iterations-this can
be easily seen numerically by varying $N_{s}$. The influence of the high-energy on the low-energy states turns
out to be small since the addition of a new site to the chain can be viewed as a perturbation of relative
strength $\Lambda^{-1 / 2}$. This perturbation is small for large values of $\Lambda$, but for $\Lambda \rightarrow 1$ it is obvious that one has to
keep more an more states to get reliable results."

(Review article: "Numerical renormalization group method for quantum impurity systems", Bulla et al. , 2008, [BCP08])

\footnotetext{
${ }^{7}$ Actually due to even/odd effects of the finite-size spectra, the RG transformation is correctly given by $H_{N+2}=R^{2}\left(H_{N}\right)$, leading to two different flows for odd and even iterations.
} 
Thus, the success of the truncation scheme is closely related to the exponentially decreasing hopping constants $t_{n} \propto \Lambda^{-n / 2}$ in the semi-infinite chain.

It turns out that for every iteration $N$ the energy spectrum of the effective Hamiltonian $H_{N}$ is a very good approximation for the spectrum of the original Hamiltonian in an energy window around a certain energy $E_{N}$. In other words, the low energy spectrum of every effective Hamiltonian $H_{N}$ gives only a good approximation of a certain energy window of the spectrum of the original Hamiltonian. The energy window around $E_{N}$ can be related to a temperature $T_{N}$. As thermodynamic quantities at temperature $T_{N}$ are mainly influenced by energies around $E_{N}$, they can be directly calculated from the spectrum of $H_{N}$ for a temperature $T_{N}$ (see also next Section). Thus, the RG flow has to be understood effectively as reducing the temperature with every step in the iteration. Fixed points in the energy flow appear like plateaus in the spectrum plotted over iterations $N$. The low-energy spectrum, close to a fixed point, is not changing significantly any more with subsequent iterations. This also transfers to thermodynamic quantities like entropy or specific heat that show the same behaviour in that fixed point. The energy flow and the appearance of fixed points of the SIAM is further discussed in Sec. 4.2.2.

\section{Calculation of static observables}

The focus of this work as mentioned before is on spectral functions and therefore the calculation of static thermodynamic properties is just shortly presented. The exponentially decreasing hopping parameters $t_{n} \propto \Lambda^{-n / 2}$ allow to relate a certain chain length $N$ to a temperature (energy). The temperature depends on the exact definition of the renormalization scheme and is given by:

$$
T_{N} \propto \frac{D}{k_{B}} \Lambda^{-N / 2} / \bar{\beta} .
$$

The parameter $\bar{\beta}$ is usually chosen between $0.4-1.0$. Standard static quantities do not mix information from different energy scales (unlike dynamic quantities) and therefore it is sufficient to use the truncated Hamiltonian $H_{N}$ at the temperature scale $T_{N}$. Standard thermodynamic quantities can then be calculated by evaluating the thermodynamic expectation values (see Eqn. (2.6)) with $H=H_{N}$ and $\beta=\beta_{N}$. Some definitions for interesting thermodynamic quantities are given in Tab. 3.1, see also Ref. [Žo7].

\section{Spectral functions}

The calculation of dynamic correlation functions is more complicated than static quantities in the NRG. Using the Lehmann representation for the spectral function (see Eqn. (2.17)) a sum over all eigenstates of the Hamiltonian has to be performed. Due to the truncation of the approximate eigenstates after the last iteration, the remaining eigenstates are all in a very small energy regime around $\omega=0$ - no information about other frequencies is left. Using the eigenstates from previous iterations that lie around $T_{N}$ will lead to a double-counting of eigenstates. There are some regularization approaches [SSK89, BCV01], but a more elegant way is to set up a complete basis set. Converting ideas from real-time evolution within the NRG [AS05, AS06], Peters et al. [PPA06] 


\begin{tabular}{l|l}
\hline Phys. Property & Expression \\
\hline magnetic susceptibility (impurity contribution) & $\chi_{\operatorname{mag}}^{\operatorname{imp}}(T)=\frac{\left(g \mu_{B}\right)^{2}}{k_{B} T}\left(\left\langle S_{z}^{2}\right\rangle-\left\langle S_{z}^{2}\right\rangle_{0}\right)$ \\
charge susceptibility (impurity contribution) & $\chi_{\text {charge }}^{\operatorname{imp}}(T)=\frac{\left(g \mu_{B}\right)^{2}}{k_{B} T}\left(\left\langle I_{z}^{2}\right\rangle-\left\langle I_{z}^{2}\right\rangle_{0}\right)$ \\
specific Heat & $C(T)=\frac{\partial E}{\partial T}=k_{B} \beta^{2}\left(\left\langle H^{2}\right\rangle-\langle H\rangle^{2}\right)$ \\
specific Heat (impurity contribution) & $C^{\operatorname{imp}}(T)=C(T)-C_{0}(T)$ \\
entropy & $S(T)=k_{B}(\beta E+\ln Z)$ \\
entropy (impurity contribution) & $S^{\operatorname{imp}}(T)=S(T)-S_{0}(T)$ \\
\hline
\end{tabular}

Table 3.1: Definition of the used thermodynamic quantities in the way that they can be evaluated within the NRG. $\langle. .\rangle_{0}$ denotes the expectation of the semi-infinite chain without the impurity that can also be evaluated within the NRG to account for the same discretization errors as in the interacting calculation, see also Ref. [Ž07].

and independently Weichselbaum et al. [WvD07] implemented these ideas for equilibrium Green's functions. The setup of the complete basis set can be achieved by introducing the reduced density matrix $\rho_{i j}^{\text {red }}=\sum_{l}\langle m, i, l|\rho| m, j, l\rangle$. Here $m$ denotes the iteration index, $i, j$ the eigenstate at this iteration and $l$ is an index for a basis state of the rest of the chain. The Green's function can then be expressed via Eqn. (2.17) by:

$$
\begin{aligned}
G_{A B}(\omega) & =\sum_{m}^{N} \sum_{i j k}\left(\langle m, i|A| m, j\rangle \rho_{j k}^{\mathrm{red}}\langle m, k|B| m, i\rangle+\langle m, i|A| m, k\rangle \rho_{j i}^{\mathrm{red}}\langle m, k|B| m, j\rangle\right) \\
& \cdot \frac{1}{\left(\omega+i \eta+E_{i}-E_{k}\right)} .
\end{aligned}
$$

The sum over $i, j, k$ has to include at least one truncated state, $|m, i\rangle$ or $|m, k\rangle$. The calculation of the Green's function with the reduced density matrix method is performed in two steps: At first a (standard) iterative diagonalization of the chain is carried out, then in a second step the iterations are done backwards. One sets up the reduced density matrix at each step and calculates Eqn. (3.27) for that step. In the end all the delta functions in the corresponding spectral function are collected into the final result, where they are broadened to give a continuous spectral function. Here the choice for a non-symmetric Gaussian broadening function whose width increases with frequency turned out to be the best choice.

It turns out that the impurity Green's function $G_{d d}(\omega)$ can be better calculated by using the 'self-energy trick'. Using the equation of motion (Eqn. (2.13)) the self energy can be expressed by [BHP98]

$$
\Sigma_{\sigma, d}(\omega)=U \frac{\ll c_{d \sigma} c_{d-\sigma}^{\dagger}, c_{d-\sigma} c_{d \sigma}^{\dagger} \gg_{\omega}}{\ll c_{d \sigma}, c_{d \sigma}^{\dagger} \gg_{\omega}} .
$$

The Green's function can then be calculated from the self energy by [vP09]:

$$
G_{\sigma d, \sigma d}(\omega)=\frac{1}{\left.\omega-\epsilon_{d}-\Sigma_{\sigma, d}(\omega)+\Delta(\omega)\right)} .
$$


This improvement has been used in all calculations in this work.

A further extension to increase the quality of spectral functions is the interleaved method (also known as "z-averaging"). In this method several NRG calculations for different logarithmic discretizations meshes controlled by a parameter $z$ are performed. Afterwards the final spectral function is calculated by averaging over all spectral functions with different z-values. This method is very successful in removing discretization artefacts and oscillatory behaviour at $T=0$. For further details see Ref. [vP09]. This improvement has been used in some calculations in this work. (The number of z-values is given, where it is used.) 


\title{
Matrix product states and density matrix renormalization group
}

\begin{abstract}
"Since its invention in 1992 by White [Whi92, Whi93], the density-matrix renormalization group (DMRG) has firmly established itself as the currently most powerful numerical method in the study of one-dimensional quantum lattices."
\end{abstract}

(Review Article: "The density-matrix renormalization group in the age of matrix product states", Schollwöck, 2011, [Sch11])

The following introduction to the DMRG is formulated in the modern formulation of MPS and is based on the review articles [Sch05b, VMC08, Sch11]. An interactive implementation of the MPS programmed by Thomas Köhler [Kö11] with my support can be found online: http://www.theorie.physik.uni-goettingen. de/ ${ }^{\sim}$ thomas .koehler.

\section{Introduction $\quad 3.3 .1$}

The DMRG has its origin in attempts to transfer ideas from the NRG, see Sec. 3.2.3, to real space. As in the NRG, one tried to group a number of sites into a block and set up a truncation scheme that just keeps a basis consisting of the eigenvectors with the lowest energies. Afterwards one used the truncated basis to set up a Hamiltonian for a larger block that consists of two old blocks. For a single-particle Hamiltonian White and Noack [WN92] could then conclusively show that this approach will generically fail.

\footnotetext{
"When applied to quantum lattice problems in a real-space blocking form, the approach is flawed in its treatment of the boundaries of a block [WN92]. The boundary errors make quantitatively accurate results impossible for most problems."
}

(Article: "Density matrix formulation for quantum renormalization groups", White, 1992, [Whi92])

It was then mainly White's idea to come up with a new truncation scheme that uses eigenstates of the reduced density matrix with the highest weight as a new basis [Whi92, Whi93]:

"... the eigenvectors with the largest eigenvalues of the density matrix of AA are the optimal states to keep, in the sense that they most accurately reproduce the state of the lattice."

(Article: "Density matrix formulation for quantum renormalization groups", White, 1992, [Whi92])

The DMRG provides ground state energies, expectation values and (short-ranged) correlation functions for one-dimensional systems with a few hundred sites up to machine precision and therefore made the DMRG to the most accurate numerical algorithm for one-dimensional systems. After this success many extensions to the DMRG were developed to calculate dynamic correlation functions in real and frequency space at zero and finite temperatures, see Ref. [Sch11] for an overview. Furthermore it was tried to apply the DMRG to two-dimensional systems. In most of these extensions it is necessary at some point to represent quantum states with high entanglement, which do not fulfil any area-law, see Sec. 3.3.5. Therefore all of these extension are limited in either reachable times, frequency resolution, temperature or size (for 2d-systems). Nevertheless, they have provided a lot of physical insight into models simply by using available computational 
resources and finite-size (time, temperature) scaling.

Matrix product states are a very powerful variational ansatz class to approximate one-dimensional - or more generally low-entangled - quantum many-body states. The variational state is hereby expressed via a product of matrices which coefficients are the variational parameters. Though there exist some classical (higher dimensional) predecessors of the MPS by Baxter [Bax68] and even before by Kramer and Wannier [KW41], the MPS have mainly there origin in the work by Fannes, Nachtergaele and Werner [FNW92]. This development was in application to the Affleck-Kennedy-Lieb-Tasaki-model (AKLTmodel) [AKLT87] were they used a translationally invariant version of MPS so called finitely correlated states. In 1995 Östlund and Rommer [OlR95] showed that the DMRG algorithm essentially is a variational ground state search algorithm for states encoded as MPS. This insight turned out to be very fruitful for further developments. From then on one could use the very flexible and easy implementable formulation of MPS together with a very fast variational ground state search algorithm and the complete toolbox of developed methods and extensions to the DMRG. The following presentation of the formalism of MPS is based on Refs. [VMC08, Sch11, Kö11].

\section{Formalism of matrix product states}

\section{Definition of matrix product states}

A general quantum state on a lattice with $L$ sites and a local Hilbert space $\mathbb{H}_{l}=\operatorname{span}\{|\sigma\rangle\}$ with a dimension of $\operatorname{dim}\left(\mathbb{H}_{l}\right)=d$ is given by the coefficients $c_{\sigma_{1} \ldots \sigma_{L}} \in C$ :

$$
|\psi\rangle=\sum_{\sigma_{1}, \ldots, \sigma_{L}} c_{\sigma_{1} \ldots \sigma_{L}}\left|\sigma_{1}, \ldots, \sigma_{L}\right\rangle
$$

As already stated in Sec. 2.1 the number of coefficients will increase exponentially $d^{L}$ with the length $L$ of the chain. In a MPS every coefficient is given by a matrix product $c_{\sigma_{1} \ldots \sigma_{L}}=M_{\sigma_{1}} \cdot \ldots \cdot M_{\sigma_{L}}$, whereby each matrix belongs locally to a site (and physical index). In order to obtain a scalar value the first and last matrix is a vector: ${ }^{8}$

$$
|\psi\rangle=\sum_{\sigma_{1}, \ldots, \sigma_{L}} M^{\sigma_{1}} \cdot \ldots \cdot M^{\sigma_{L}}\left|\sigma_{1}, \ldots, \sigma_{L}\right\rangle
$$

Every quantum state can be expressed exactly by a MPS, but the matrix dimension will in general increase exponentially with $L$. The exact transformation of a given quantum state into MPS form is described in Appendix A.1.1. In order to work with MPS the matrix dimension is usually limited to a certain maximum dimension $m$. This can be understood as an approximation scheme for a given quantum state: The coefficients of the matrices are searched that give the best approximation of a given quantum state. In a different perspective MPS with a limited matrix dimension can represent only a (small) subset of states in the Hilbert space. It turned out that this subspace consists of (spatially)

\footnotetext{
${ }^{8}$ This is just the case for open boundary conditions, for periodic boundary conditions the trace of the matrix product is taken.
} 
low-entangled states. This can be seen immediately as for a matrix dimension of $m=1$ only (spatial) product states can be represented exactly. By increasing the matrix dimension entanglement between different lattice sites can be introduced to the MPS. Ground states of (gapped) one-dimensional quantum systems only show low-entanglement and can therefore be nicely represented (and approximated) with MPS. Further discussion is postponed to Sec. 3.3.5.

The MPS representation of a state is not unique as one can always insert an identity between to matrices in a MPS: $M^{\sigma_{i}} \mathbb{1} M^{\sigma_{i+1}}=M^{\sigma_{i}} X X^{-1} M^{\sigma_{i+1}}=M^{\prime \sigma_{i}} M^{\prime \sigma_{i+1}}$. This property of the MPS gives raise to some gauge freedom. If the matrices fulfil the condition $\sum_{\sigma_{l}} A^{\sigma_{l} \dagger} A^{\sigma_{l}}=\mathbb{1}$, they are called left-normalized. One can also construct a MPS with right-normalized matrices, $\sum_{\sigma_{l}} B^{\sigma_{l}} B^{\sigma_{l} \dagger}=\mathbb{1}$. A mixed-normalized state is given by $|\psi\rangle=\sum_{\sigma} A^{\sigma_{1}} \cdots A^{\sigma_{l-1}} M^{\sigma_{l}} B^{\sigma_{l+1}} \cdots B^{\sigma_{L}}|\boldsymbol{\sigma}\rangle .^{9}$ In the following introduction open boundary conditions (obc) are always assumed. Computations for periodic boundary conditions (pbc) are a little bit more subtle as the computational costs are usually higher. This is due to the fact that contractions for obc can be done usually in a more efficient way. ${ }^{10}$ Furthermore, efficient implementations of MPS have to include the usage of symmetries, see Refs. [MG02, SZV10, Sch11] for further details.

\section{Scalar product}

The scalar product of two quantum states expressed as MPS can be calculated by:

$$
\begin{aligned}
\langle\phi \mid \psi\rangle & =\sum_{\boldsymbol{\sigma} \boldsymbol{\sigma}^{\prime}}\left\langle\boldsymbol{\sigma}^{\prime}\left|\tilde{M}^{\sigma_{1}^{*}} \cdots \tilde{M}^{\sigma_{L}^{\prime} *} M^{\sigma_{1}} \cdots M^{\sigma_{L}}\right| \boldsymbol{\sigma}\right\rangle \\
& =\sum_{\boldsymbol{\sigma}} \tilde{M}^{\sigma_{1} *} \cdots \tilde{M}^{\sigma_{L^{*}}} M^{\sigma_{1}} \cdots M^{\sigma_{L}} .
\end{aligned}
$$

The evaluation of this expression is computationally very inefficient as one has to perform $d^{L} \cdot(d L-1)$ matrix-multiplications. That means, that the complexity will increase exponentially with chain length. But the expression can be reformulated, so that only $d L$ additions and $d(L-1)$ matrix-multiplications are needed, which just scales polynomially in system size:

$$
\langle\phi \mid \psi\rangle=\sum_{\sigma_{L}} \tilde{M}^{\sigma_{1} \dagger}\left(\cdots\left(\sum_{\sigma_{2}} \tilde{M}^{\sigma_{2} \dagger}\left(\sum_{\sigma_{1}} \tilde{M}^{\sigma_{1} \dagger} M^{\sigma_{1}}\right) M^{\sigma_{2}}\right) \cdots\right) M^{\sigma_{L}}
$$

Therefore calculating scalar products between MPS is usually only of minor computational effort.

\footnotetext{
${ }^{9}$ In this work left-normalized matrices are denoted by the letter $A$, right-normalized matrices by the letter $B$ and non-normalized matrices by the letter $M$.

${ }^{10}$ For pbc the matrices cannot all be right or left-normalized which is the main problem to perform efficient contractions.
} 


\section{Matrix product operators}

Analogously to quantum states, the operators can also be expressed as matrix product operators (MPO). They are defined in a similar way by:

$$
\hat{O}=\sum_{\boldsymbol{\sigma}, \boldsymbol{\sigma}^{\prime}} c_{\left(\sigma_{1} \ldots \sigma_{L}\right)\left(\sigma_{1}^{\prime} \ldots \sigma_{L}^{\prime}\right)}|\boldsymbol{\sigma}\rangle\left\langle\boldsymbol{\sigma}^{\prime}\left|=\sum_{\boldsymbol{\sigma}, \boldsymbol{\sigma}^{\prime}} W^{\sigma_{1} \sigma_{1}^{\prime}} \ldots W^{\sigma_{L} \sigma_{L}^{\prime}}\right| \boldsymbol{\sigma}\right\rangle\left\langle\boldsymbol{\sigma}^{\prime}\right| .
$$

The $W$-matrices are similar to the $M$-matrices of MPS, but they have two physical indices. The construction of the $W$-matrices is straight-forward for local operators. Even for shortranged Hamiltonians there is a very efficient way to construct the Hamiltonians with a very low matrix dimension for the $W$-matrices. The explicit construction of the Hamiltonian as a MPO is described in Appendix A.1.2. The application of a MPO on a MPS leads to a new MPS:

$$
\begin{aligned}
\hat{O}|\psi\rangle & =\sum_{\boldsymbol{\sigma}, \boldsymbol{\sigma}^{\prime}, \boldsymbol{\sigma}^{\prime \prime}}\left(W^{\sigma_{1} \sigma_{1}^{\prime}} \ldots W^{\sigma_{L} \sigma_{L}^{\prime}}\right)\left(M^{\sigma_{1}^{\prime \prime}} \cdots M^{\sigma_{L}^{\prime \prime}}\right)|\boldsymbol{\sigma}\rangle \underbrace{\left\langle\boldsymbol{\sigma}^{\prime} \mid \boldsymbol{\sigma}^{\prime \prime}\right\rangle}_{\delta_{\sigma^{\prime} \sigma^{\prime \prime}}} \\
& =\sum_{\boldsymbol{\sigma}, \boldsymbol{\sigma}^{\prime}}\left(W^{\sigma_{1} \sigma_{1}^{\prime}} \ldots W^{\sigma_{L} \sigma_{L}^{\prime}}\right)\left(M^{\sigma_{1}^{\prime}} \cdots M^{\sigma_{L}^{\prime}}\right)|\boldsymbol{\sigma}\rangle=\sum_{\boldsymbol{\sigma}} \tilde{M}^{\sigma_{1}} \cdots \tilde{M}^{\sigma_{L}}|\boldsymbol{\sigma}\rangle=|\tilde{\psi}\rangle .
\end{aligned}
$$

The values of the new $\tilde{M}$ matrices are given by:

$$
\tilde{M}^{\sigma_{i}}=\sum_{\sigma_{i}^{\prime}} W_{b_{i-1}, b_{i}}^{\sigma_{i} \sigma_{i}^{\prime}} M_{a_{i-1}, a_{i}}^{\sigma_{i}^{\prime}}
$$

The new MPS $|\tilde{\psi}\rangle$ has an increased matrix dimension (the matrix dimensions multiply). An expectation value of an operator can be calculated by:

$$
\langle\psi|\hat{O}| \psi\rangle=\langle\psi \mid \tilde{\psi}\rangle
$$

The computational costs of the calculation of a expectation value are of the order $N d^{2} m^{3}$ for open boundary conditions. For periodic boundary conditions the computational costs are of the order $N d^{2} m^{5}$.

\section{Summation of matrix product states}

Implementing the Lanczos algorithm in MPS, one is confronted with the summation of several MPS. The summation of two MPS $|\Psi\rangle$ and $|\Phi\rangle$ will lead to a new MPS $|\chi\rangle$ :

$$
\begin{aligned}
|\chi\rangle & =|\Psi\rangle+|\Phi\rangle=\sum_{\sigma_{1}, \ldots, \sigma_{L}} M^{\sigma_{1}} \cdot \ldots \cdot M^{\sigma_{L}}\left|\sigma_{1}, \ldots, \sigma_{L}\right\rangle+\sum_{\sigma_{1}, \ldots, \sigma_{L}} N^{\sigma_{1}} \cdot \ldots \cdot N^{\sigma_{L}}\left|\sigma_{1}, \ldots, \sigma_{L}\right\rangle \\
& =\sum_{\sigma_{1}, \ldots, \sigma_{L}} \tilde{M}^{\sigma_{1}} \cdot \ldots \cdot \tilde{M}^{\sigma_{L}}\left|\sigma_{1}, \ldots, \sigma_{L}\right\rangle .
\end{aligned}
$$

The new matrices are given by a direct sum $\tilde{M}^{\sigma_{i}}=M^{\sigma_{i}} \bigoplus N^{\sigma_{i}}$. Just the first and last matrix, that are actually vectors for obc are given in a special manner by a row vector $[M N]$ and column vector $[M N]^{\dagger}$, see Ref. [Sch11] for details. It should be emphasized that the dimension of the matrices under the summation add. Therefore MPS with a fixed matrix dimension do not form a vectorspace. 


\title{
Ground state search 3.3 .3
}

\begin{abstract}
"An attractive feature of tensor network states is that they are largely unbiased variational ansätze, in the sense that they are capable of representing many different types of ground states through a proper choice of variational parameters, as clearly witnessed by two decades of MPS explorations with DMRG."
\end{abstract}

(Article: "Tensor network states and geometry", Evenbly and Vidal, 2011, [EV11])

Up to now just the states and the operators in matrix product form and their basic operations have been defined. One of the major milestones in the last years was to realize that the DMRG algorithm gives the (approximate) ground state of one-dimensional systems as a MPS [OlR95, DMDNS98, VPC04]. ${ }^{11}$ This insight led to the understanding that the DMRG is actually using the time-independent variational principle ${ }^{12}$ to search for the state with the lowest energy in the manifold of the variational states spanned by MPS. One distinct feature of the DMRG is the local optimization routine, that allows to handle even large matrix dimensions with low computational effort.

\section{Time-independent variational principle}

As introduced in Sec. 2.2, the energy of a physical system in state $|\Psi\rangle \in \mathbb{H}$ is given by the expectation value of the Hamiltonian

$$
E=\frac{\langle\Psi|H| \Psi\rangle}{\langle\Psi \mid \Psi\rangle}
$$

This definition of the energy allows for the direct application of the time-independent variational principle. The idea is to define a manifold of variational states by $\mathcal{V}=$ $\operatorname{span}\left\{\left|\Psi^{\mathcal{V}}(y)\right\rangle, \forall y=\left(y_{1}, y_{2}, \ldots\right), y_{i} \in \mathbb{C}, \forall i\right\}$. These states are parametrized by a finite or infinite set of complex number $y_{i} \cdot{ }^{13}$ The energy of the state(s) with the lowest energy in this manifold will set an upper bound for the exact ground state energy of the system in the total Hilbertspace:

$$
E_{0} \leq E_{0}^{\mathcal{V}}=\min _{\left|\Psi^{\mathcal{V}}(y)\right\rangle \in \mathcal{V}}\left(\frac{\left\langle\Psi^{\mathcal{V}}(\bar{y})|\mathcal{H}| \Psi^{\mathcal{V}}(y)\right\rangle}{\left\langle\Psi^{\mathcal{V}}(\bar{y}) \mid \Psi^{\mathcal{V}}(y)\right\rangle}\right)
$$

This minimalization problem can be solved in the standard way: Setting the derivatives with respect to the parameters $y$ to zero and solving these equations for the optimal parameters $y_{0}$. Choosing the trial wavefunction to be an expansion in an orthonormal basis set with coefficients $y_{i}$, this is called the Rayleigh-Ritz [Ray70, Rit08] variational method.

\footnotetext{
${ }^{11}$ Actually just the one-site DMRG algorithm [Whi05] gives a MPS - see Ref. [Sch11] for further details.

${ }^{12}$ The explizit notion of the "time-independent" variational principle is done as in a very recent work $\left[\mathrm{HCO}^{+} 11\right]$ the time-dependent variational principle has been used to calculate dispersion relations.

${ }^{13}$ For a fixed MPS dimension this set will be finite.
} 
Expanding the manifold of variational states by increasing the number of parameters one actually has to prove the convergence, but one can also keep it in the spirit of Ritz:

"Nach dem vorliegenden Beispiel [...die Berechnung der Schwingungszahl des Fundamentaltons einer Seite...]
dürfte der Physiker bei der Anwendung des neuen Rechnungsverfahrens auch in Fällen, wo der theoretische
Konvergenzbeweis zunächst noch fehlt, sich durch diesen Mangel nicht allzusehr beunruhigt fühlen." 14
(Article: "Über eine neue Methode zur Lösung gewisser Variationsprobleme der mathematischen Physik",
Ritz, 1908,[Rit08])

Furthermore one has to keep in mind, that one can find a state with an energy that is very close to the real ground state energy, but that can give different behaviour for the expectation values of other variables, e.g. magnetization, than the true ground state.

\section{DMRG ground state search}

The DMRG algorithm can be interpreted as the application of the time-independent variational principle to the MPS as variational states. Therefore the minimalization problem as stated in Eqn. (3.40) has to be solved with MPS. This problem can be computed using the Lagrange-formalism. This leads to the problem of minimizing $\langle\psi|H| \psi\rangle$ under the constraint that the norm $\langle\psi \mid \psi\rangle$ is constant. Introducing the ground state energy $\lambda=E_{0}$ as the Lagrange-multiplier, one gets the Lagrange function:

$$
L=\langle\psi|\hat{H}| \psi\rangle-E_{0}(\langle\psi \mid \psi\rangle-1)
$$

The usual way to continue is to differentiate the Lagrange function for all $p$ parameters of the trial wavefunction and set the derivatives to zero. This will lead to $p+1$ coupled equations that have to be solved consistently. Within MPS there are $d \cdot L$ matrices with $m^{2}$ coefficients. This makes $p=d \cdot L \cdot m^{2}$ parameters in total and therefore the global optimization will just work for very small matrix dimensions $m$. The DMRG algorithm now works as follows: Just optimize one matrix of the MPS at a time and sweep through the system optimizing the matrices one after each other. In numerical mathematics this form of the optimization is known as alternating least-squares (ALS), see Refs. [VMC08, PTVF92].

In Appendix A.1.3 local forms for the expectation value of $H$ and the norm with respect to a single matrix $M^{\sigma_{l}}$ at site $l$ are derived. Using the variational principle the Lagrange function has to be differentiated with respect to all coefficients $M_{a_{l-1}, a_{l}}^{\sigma_{l}}$ and then these derivatives are set to zero:

$$
\begin{aligned}
0 & \stackrel{!}{=} \frac{\partial L}{\partial M_{a_{l-1}, a_{l}}^{\sigma_{L}}}=\frac{\partial}{\partial M_{a_{l-1}, a_{l}}^{\sigma_{L}}}(\langle\psi|\hat{H}| \psi\rangle)-\lambda \frac{\partial}{\partial M_{a_{l-1}, a_{l}}^{\sigma_{L}}}(\langle\psi|| \psi\rangle) \\
& =\sum_{\sigma_{l}^{\prime}} \sum_{a_{l-1}^{\prime}, a_{l}^{\prime}} \sum_{b_{l-1}, b_{l}} L_{a_{l-1}^{\prime}, b_{l-1}}^{a_{l-1}} W_{b_{l-1}, b_{l}}^{\sigma_{l}, \sigma_{l}^{\prime}} R_{a_{l}^{\prime}, b_{l}}^{a_{l}} M_{a_{l-1}^{\prime}, a_{l}^{\prime}}^{\sigma_{l}^{\prime}}-\lambda \sum_{a_{l-1}^{\prime}, a_{l}^{\prime}} M_{a_{l-1}^{\prime}, a_{l}^{\prime}}^{\sigma_{l}^{\prime}} .
\end{aligned}
$$

Here the $L$ and $R$ matrices are basically matrix products of the matrices on the left and right side of the regarded $M$-matrix. The $W$-matrix is the local MPO matrix of the

\footnotetext{
${ }^{14}$ (Free) English translation:"With the present example [... the calculation of the wave number of a fundamental mode ... ] the physicist may also not to be too much concerned with the application of the new calculation method also in those cases where the theoretical proof of convergence is still missing."
} 
Hamiltonian (see Appendix A.1.3 for further details). This last line allows to introduce an effective Hamiltonian $H_{\mathrm{Eff}}$ by:

$$
H_{\mathrm{Eff}\left(\sigma_{l} a_{l-1} a_{l}\right)\left(\sigma_{l}^{\prime} a_{l-1}^{\prime} a_{l}^{\prime}\right)}=\sum_{b_{l-1}, b_{l}} L_{a_{l-1}^{\prime}, b_{l-1}}^{a_{l-1}} W_{b_{l-1}, b_{l}}^{\sigma_{l}, \sigma_{l}^{\prime}} R_{a_{l}^{\prime}, b_{l}}^{a_{l}}
$$

and $\nu_{\sigma_{l} a_{l-1} a_{l}}=M_{a_{l-1}, a_{l}}^{\sigma_{l}}$. Finally, the local optimization of a matrix is transformed to a local eigenvalue problem that has to be solved:

$$
H_{\mathrm{Eff}} \nu-\lambda \nu=0 \text {. }
$$

This eigenvalue problem can be solved exactly (or approximately by a Lanczos) and the smallest eigenvalue gives the optimized value for the overall ground state energy. The related eigenvector $\nu_{0}$ gives the new optimized matrix: $M^{\sigma_{L}}$. In order to optimize a given random starting state $|\psi\rangle$ iteratively to represent the ground state, one sweeps trough the chain optimizing one matrix after the other. The technical details are explained in Appendix A.1.3.

Local optimization routines are usually considered to be very problematic as they are known to get stuck in local minima [PTVF92]. This is also the case for the DMRG optimization for certain Hamiltonians especially with long-range interactions or disordered systems. The original two-site version of the DMRG optimized two sites at the same time and by this is more robust towards these problems. Within MPS there are several workarounds to cope with these problems, see Ref. [Sch11]. Different approaches to obtain the ground state were also developed. One idea is to use multi-grid algorithms [DBTR12] and by this give up the local optimizations. Another idea is to use imaginary time evolution of a random initial state that converges nicely towards the ground state if the Hamiltonian has a sufficiently large gap. ${ }^{15}$ For the systems considered in this work convergence to the ground state was not a problem.

\section{Compression of matrix product states}

As denoted beforehand the addition of several MPS and the application of a MPO to a MPS increases the matrix dimension of the new MPS. Repetitive applications of these operations - like in the Lanczos recursion formula - will therefore lead to MPS with a huge matrix dimension that cannot be handled any more. A compression of the increased matrix dimension is mandatory. There are two existing algorithms for compressing a MPS:

\section{- SVD compression}

Within the SVD compression each matrix is iteratively decomposed by a singular value decomposition (SVD). The new matrices of the SVD are reshaped to the new matrix dimension $d^{\prime}$ by truncating the diagonal matrix to the $d^{\prime}$ largest singular values. In a left sweep this is shown for a matrix $M^{\sigma_{k}}$ as an example:

$$
M_{i j}^{\sigma_{k}}=M_{i,\left(\sigma_{k}, j\right)}=\sum_{l}^{d} U_{i l} S_{l l} V_{l, \sigma_{k} j} \approx \sum_{l}^{d^{\prime}} U_{i l} S_{l l} B_{l j}^{\sigma_{k}} .
$$

\footnotetext{
${ }^{15}$ The time needed is longer than the inverse gap [VMC08].
} 
The orthogonal $V$-matrix of the SVD is reshaped into the new (right-orthogonalized) $B^{\sigma_{k}}$-Matrix and the product of $U S$ is multiplied to the next $M^{\sigma_{k-1}}$-matrix that will be decomposed in the next step. Within SVD compression a few compression sweeps are necessary, but the SVD compression may also get stucked in a local minimum.

- Variational compression

In variational compression one tries to minimize the distance between the exact state $|\Psi\rangle$ and the approximate state $\left|\Psi^{\prime}\right\rangle$ with smaller matrix dimension $d^{\prime}$ :

$$
\||\Psi\rangle-\left|\Psi^{\prime}\right\rangle \|^{2}=\langle\Psi \mid \Psi\rangle-\left\langle\Psi^{\prime} \mid \Psi\right\rangle-\left\langle\Psi \mid \Psi^{\prime}\right\rangle+\left\langle\Psi^{\prime} \mid \Psi^{\prime}\right\rangle
$$

This minimization problem can again be solved by a local optimization scheme in the spirit of the DMRG. While sweeping through the system one optimizes always a single matrix $M^{\prime \sigma_{k}}$ of the state $\left|\Psi^{\prime}\right\rangle$. At every step the derivatives with respect to all the entries of the $M^{\prime \sigma_{k}}$ have to be computed and set to zero. This will lead to a big linear equation system that has to be solved and gives a new matrix $\tilde{M}^{\prime \sigma_{k}}$. With variational compression also a few compression sweeps are needed.

For more details on these approaches please see Ref. [Sch11]. In this work the variational compression has been used.

\section{Entanglement entropy 3.3 .5}

"... In contrast to [classical] thermal states this entropy [in quantum mechanical systems] does not originate
from a lack of knowledge about the microstate of the system. Even at zero temperature we encounter a non-
zero entropy. This entropy arises because of a fundamental property of quantum mechanics: entanglement.
This quite intriguing trait of quantum mechanics gives rise to correlations even in situations where the
randomness cannot be traced back to a mere lack of knowledge."

(Review Article: "Colloquium: Area laws for the entanglement entropy", Eisert et al. , 2010, [ECP10])

The success of the DMRG and MPS could be mainly explained by developments not from the condensed matter area, but from the field of quantum information: The study of the entanglement entropy. The amount of entanglement between a region $A$ of a lattice and the rest $B$ of the lattice is given by the entanglement entropy:

$$
S(A)=-\operatorname{tr}\left(\rho_{A} \log _{2} \rho_{A}\right) .
$$

Here $\rho_{A}$ denotes the reduced density matrix of the ground state $\left|E_{0}\right\rangle$ which can be calculated by tracing out the degrees of freedom of the rest of the lattice $B$ :

$$
\rho_{A}=\operatorname{tr}_{B}\left|E_{0}\right\rangle\left\langle E_{0}\right| \text {. }
$$

Analyses of the entanglement entropy scaling of general lattice Hamiltonians found remarkable universal properties that are summarized with the term 'area laws', see for example Refs. [Sre93, VLRK03, PEDC05, BHV06, EO06, EV11], a comprehensive review is given by Ref. [ECP10]. The main results of these studies are that the entanglement entropy of ground states of Hamiltonians with local interactions is scaling with the surface (area) of the region $A$ - and not with the volume. For one-dimensional systems this means that the entanglement entropy stays constant with increasing system size. In Fig. 3.3 the 
a)

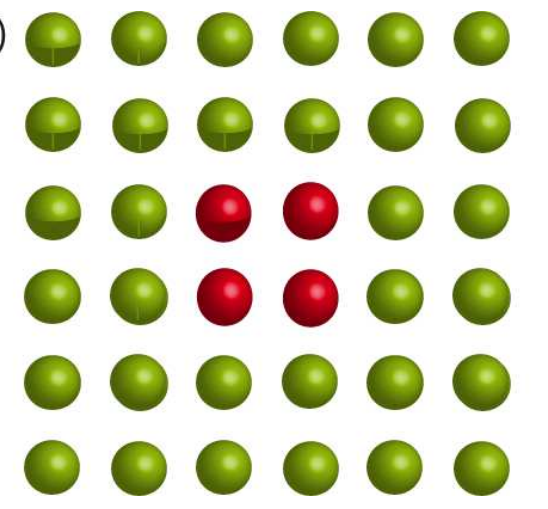

b)

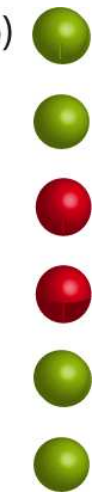

Figure 3.3: The entanglement entropy for non-critical ground states scales with the surface area of the considered region A (here marked in red). The scaling of the surface is dependent on the dimensionality: a) $2 \mathrm{~d}$ square lattice: Increasing the region $A=L \cdot L$ to $A=(L+1) \cdot(L+1)$, the surface $S=4 L-4$ scales linearly: $4(L+1)-4$. b) $1 \mathrm{~d}$ : Increasing the region $A=L$, the surface $S=2$ stays constant.

surface scaling for a one and two-dimensional system is visualized.

However, it must be distinguished between critical and non-critical systems. ${ }^{16}$ For critical systems there are logarithmic corrections with the system size. The results of the analyses of the entanglement entropy scaling are summarized in Tab. 3.2. In order to represent ground states with variational states like MPS the central question is whether they can incorporate the discussed entanglement scaling. It could be shown that the entanglement entropy of an MPS stays constant if the system size is increased (and $m$ kept constant) and therefore they fulfil the area law [Has07a, Has07b] for one-dimensional systems. That means that for a non-critical Hamiltonian by increasing the system size the number of local parameters $m$ can be held constant to keep the same precision in the ground state properties, which is meant by the statement "Matrix product states represent ground states faithfully" [VC06]. The behaviour of a critical one-dimensional system can (in principle) not be captured by MPS. However, all these statements have to be interpreted with care as they are usually derived for general lattice Hamiltonians in the thermodynamic limit. Usually the constant entropy scaling is not seen for small finite systems, especially in the neighbourhood of criticality and on the other hand even ground states of critical systems can usually be well represented:

"Thus, while the MPS can accurately approximate short-range properties of a critical ground state, it
necessarily fails to capture its properties at asymptotically large distances. [In practice, however, the cost
of MPS-based approaches scales only as $O(3)$ with the bond dimension $D$. This means that one can use a
very large value of $D$, which often allows the critical behavior of a system to be accurately captured up to
some very large length scale.]"

(Review article: "Matrix product states, projected entangled pair states, and variational renormalization group methods for quantum spin systems", Verstraete et al. , 2008, [VMC08])

There have been developments of classes of variational ansatz states that incorporate the entanglement entropy scaling as given in Tab. 3.2 also for higher dimensions and at criticality. For critical systems (especially in one dimension) the multiscale entanglement renormalization ansatz (MERA) [Vid07, Vid08] turned out to give the right long-distance behaviour of static correlation functions. Projected entangled pair states (PEPS) [VC04, MVC07] is the natural extension of MPS for non-critical two dimensional systems. However, MERA and PEPS suffer from a high computational complexity and their full potential is still unexplored.

\footnotetext{
${ }^{16}$ Critical systems are at or close to a continuous phase transition.
} 


\begin{tabular}{ll|lllll|}
\hline & & \multicolumn{5}{|c}{ Fermi surface } \\
& & gapped & $\Gamma=0$ & $\Gamma=1$ & $\Gamma=2$ \\
\hline \multirow{2}{*}{ Space dimension } & $\mathrm{d}=1$ & const & $\log _{2} L$ & - & - \\
& $\mathrm{d}=2$ & $L$ & $L$ & $L \log _{2} L$ & - \\
& $\mathrm{d}=3$ & $L^{2}$ & $L^{2}$ & $L^{2}$ & $L^{2} \log _{2} L$ \\
\hline
\end{tabular}

Table 3.2: (Simplified) Scaling of the entanglement entropy of ground states of local Hamiltonians with the area size $L$ for different spatial dimensions $d$ with respect to the criticality of the system expressed by the dimension $\Gamma$ of the Fermi surface. See Ref. [EV11] for further details.

The focus in this work is on spectral functions. For these also excited states have to be represented by MPS. For excited states general statements of the scaling of the entanglement entropy have not been explored in detail so far [ECP10]. There are some hints that the area laws as derived for the ground states should also hold for the low-lying excited states [Req06, AFC09]. Within the implementation of the Lanczos algorithm in MPS the excited states are not directly represented by a single MPS, but by a sum of Lanczos vectors represented by MPS. Therefore the success of the Lanczos algorithm in MPS is linked to the ability of MPS to represent Lanczos states, which is directly related to the amount of entanglement of the Lanczos states. The further discussion is postponed to Sec. 5.2.1.

\section{Subtle differences $\quad 3.3 .6$}

In the previous section the DMRG in the formulation of MPS was introduced. It was emphasized that the (single-site) original DMRG is in principle equivalent to the variational ground state search with MPS. However, there are two differences that are important when implementing the Lanczos recursion formula given by Eqn. (3.2) for the calculation of spectral functions. In the Lanczos algorithm several (three) Lanczos states have to be calculated simultaneously. In the original DMRG, the problem of an acceptable approximation for several states at one time is solved by the procedure of multi-targeting: the reduced density operator that governs the choice of reduced bases in DMRG is set up from a weighted sum of reduced density operators formed for each state individually:

$$
\rho_{A}=\sum_{i} \alpha_{i}\left|\Psi_{i}\right\rangle\left\langle\Psi_{i}\right|
$$

The weights $\alpha_{i}$ are usually chosen to be the same for all states. This implies that none of the states will be represented with the accuracy the method could have achieved for it alone:

\footnotetext{
"This can of course only be done at a certain loss of accuracy for given numerical resources and for a few states only. At the price of calculating the contractions anew for each state, in the MPO/MPS formulation, the state bases are only tied up at the level of the exact full basis. MPO/MPS formulations therefore acquire their full potential versus conventional DMRG language once multiple states get involved."
} 
In the MPS formulation, each state carries its own implicit (optimal) choice of reduced basis, hence is approximated more precisely; moreover, the formalism automatically deals with the fact that each state has been approximated differently.

The second advantage is due to the introduction of MPOs. With MPOs the Hamiltonian can be expressed exactly whereas in DMRG it is expressed in the reduced basis chosen by DMRG to represent the state(s) of interest. Due to the frequent application of the Hamiltonian in the recursion equation (see Eqn. (3.2)) this has significant impact.

\footnotetext{
"Another instance where the MPO/MPS formulation is superior, albeit at elevated numerical cost, is the calculation of the expression $\left\langle\Psi_{i}\left|H^{2}\right| \Psi_{i}\right\rangle$, which is interesting e.g. in the context of estimating how accurately a ground state has been obtained. In the MPO formalism, it can be done exactly up to the inherent approximations to $\left|\Psi_{i}\right\rangle$."
}

(Review Article: "The density-matrix renormalization group in the age of matrix product states", Schollwöck, 2011, [Sch11])

\section{Spectral functions 3.3 .7}

Calculating dynamic correlation functions with the DMRG/MPS has a long tradition that dates back to the first approach by Hallberg in 1995 [Ha195]. In the next section different approaches to dynamic correlation functions with MPS/DMRG are presented, whereby the Lanczos algorithm with adaptive DMRG and the Lanczos algorithm with MPS have been developed within this work and published in Refs. [DHP $\left.{ }^{+} 11, \mathrm{DWH}^{+} 12\right]$.

\section{Lanczos algorithm with standard DMRG}

The idea to use the Lanczos algorithm within standard DMRG was first put forward by Hallberg [Hal95] following the procedure outlined in Sec. 3.1.2, with $\left|E_{0}\right\rangle$ provided by a ground state DMRG calculation. The operations $H|\psi\rangle$ are provided in DMRG such that the Lanczos algorithm can be implemented easily. Even if one assumes that $\left|E_{0}\right\rangle$ is available at precision close to exact diagonalization, which is true to a very good approximation, an additional approximation of this approach is that in the calculation of the Lanczos vectors (essentially $H^{n}\left|f_{0}\right\rangle$ ) $H$ is available only approximately and usually optimized for the operation $H\left|E_{0}\right\rangle$, see Sec. 3.3.6. DMRG, if applied naively, will therefore introduce increasingly severe and systematic errors for the higher Lanczos vectors. A partial remedy is provided by the multi-targeting procedure, by which one finds a good approximate representation of several Lanczos vectors (which changes also the approximations made in the representation of $H$ ). The quandary of this approach is that if one targets few Lanczos vectors, those are represented quite accurately, but all others quite badly; if one targets many Lanczos vectors, all are represented at similar, but quite low accuracy. Loss of orthogonality occurs as in every Lanczos procedure, but can be mended by reorthogonalization of the Lanczos vectors; as DMRG keeps them in a common basis representation, this can be done easily, but the price is the low accuracy of each individual Lanczos vector due to multiple targeting, see Sec. 3.3.6. 
Mostly the spectral function is presented as a smooth curve with an artificial broadening, but not as the set of poles and weights naturally following from the Lanczos representation. This makes sense as the accuracy of individual pole positions and spectral weights is not so high in view of the systematic errors. Therefore it has found numerous successful applications e.g. in Refs. [Nis99, ZJW99, YH00, OAAY01, GHR04].

\section{Lanczos algorithm with adaptive DMRG}

To overcome the multi-target approach in the standard DMRG framework an adaptive algorithm was newly developed $\left[\mathrm{DHP}^{+} 11\right]$. The Lanczos vectors are still calculated in a multi-targeting approach, but the Lanczos vectors to be targeted are restricted to the last three ones which occur in the recursion. This implies a continuous change of basis as the algorithm evolves. As the basis in the standard DMRG framework always changes one has to use the so-called wavefunction updates [Whi96]. In Fig. 3.4 a comparison between

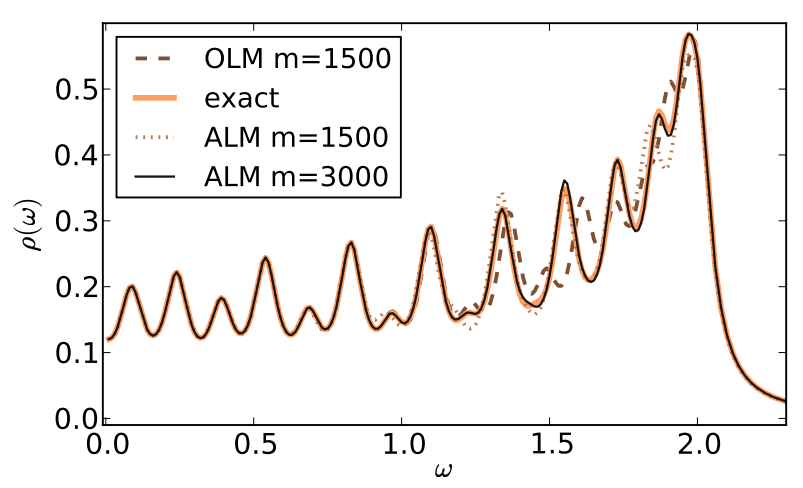

Figure 3.4: (Published in $\left[\mathrm{DHP}^{+} 11\right]$.) Broadened spectral function for spinless fermions $(L=40, \eta=$ 0.05, 200 Lanczos vectors) calculated with the new adaptive implementation (ALM), the original implementation (OLM) and compared to the exact solution given via Eqn. (3.51). The DMRG truncation number is given by $m$.

the original Lanczos method and the new adaptive method is shown for spinless fermions with Hamiltonian:

$$
H_{\text {free }}=-t \sum_{i=1}^{L} c_{i+1}^{\dagger} c_{i}+c_{i}^{\dagger} c_{i+1}
$$

$c_{i}^{\dagger}$ and $c_{i}$ are the usual creation and annihilation operators of spinless fermions with hopping parameter $t$. The (broadened) LDOS measured in the middle of a chain $(x=$ $L / 2$ ) of non-interacting spinless fermions is compared to the exact solution, which can be obtained from the Green's function in the single-particle picture, see Ref. [Dar09] for details:

$$
\rho(\omega+i \eta, x)=-\frac{1}{\pi} \operatorname{Im} G_{x x}(\omega+i \eta), \quad G_{i j}^{-1}= \begin{cases}\omega+i \eta, & \text { if } i=j \\ -t, & \text { if } i=j+1 \text { or } i=j-1 \\ 0, & \text { else }\end{cases}
$$

One can clearly see that the new adaptive method better reproduces the exact solution than the old Lanczos method. It must be stated however, that this approach is potentially vulnerable to the ever-present problem of Lanczos "ghosts", the appearance of spurious eigenvalues due to loss of orthonormality between Lanczos vectors. The adaptive basis changes make reorthogonalization schemes very difficult if not effectively impossible. 


\section{Lanczos algorithm with MPS}

As already stated in Ref. $\left[\mathrm{DHP}^{+} 11\right]$ the adaptive Lanczos method can be further improved by working directly with MPS. Every Lanczos state is represented by one MPS and the only approximation comes from the compression of the state. Choosing the MPS formulation of the DMRG in the Lanczos method will completely eliminate the need for multiple targeting, giving additional accuracy. Furthermore an exact representation of $H$ is achieved due to the MPO representation and the possibility of a reorthogonalization scheme, lost in the adaptive Lanczos method, is regained.

The algorithm proceeds exactly as before, but the ground state $\left|E_{0}\right\rangle$ is written in MPS representation and $H$ is written as a MPO. Considering the recursion relation, one has to keep in mind that both the application of $H$ to a state and the addition of two MPS (as occurs in the Lanczos procedure) lead, if carried out exactly, to a new MPS, but with a dimension that is larger than that of the original MPS: under summation, dimensions add; under application of an MPO to an MPS, the dimensions multiply, see Sec. 3.3.2. This is to be seen in contrast to the same operations in the conventional DMRG framework, where these operations do not increase the numbers of DMRG states to be kept (i.e. the MPS matrix dimension), at the price of an approximate representation of the Hamiltonian $H$ which is adapted only to specific Lanczos states and introduces quite severe inaccuracies for Lanczos states where $H$ has been applied often. It is at this point that MPS based Lanczos dynamics has to introduce its only approximation, namely the compression of the large-dimension MPS back to the original dimension at some loss of accuracy. This compression is carried out iteratively until the compressed state has become stable, see Sec. 3.3.4. This approximation avoids the systematic errors due to an approximate Hamiltonian and is in practice less severe. Multiple targeting is eliminated.

However, the approximation due to the compression of the Lanczos states still leads to the ghost problem. In the MPS approach, this problem is not as bad as in the adaptive DMRG approach, but it is still exacerbated in comparison to the standard DMRG Lanczos method. The advantage is that this problem can be cured in comparison to the inexact Hamiltonian representation. Attempts with partial and total re-orthogonalization were not successful due to the rather large overlap $\left\langle f_{k} \mid f_{n}\right\rangle$ of the different Lanczos vectors. The current Lanczos vector is changed significantly by this procedure, which results in an even worse representation of the Hamiltonian. Alternatively speaking, the fact that reorthogonalization involves sums implies again compression, which will make minor new approximations only if the reorthogonalization is mild. However an ex-post reorthogonalization scheme which does not change the Lanczos vectors explicitly turned out to be quite successful.

In the standard Lanczos the matrix elements between Lanczos vectors $\left\langle f_{i}|H| f_{j}\right\rangle$ are zero for $i>j+1$ and $i<j-1$ leading to the tridiagonal effective matrix, see Eqn. (3.3). Introducing numerical errors the Lanczos vectors are not (perfectly) orthogonal any more and therefore also the matrix is not (perfect) tridiagonal anymore. In the usual Lanczos algorithm this is either ignored or the vectors are reorthogonalized and by this the tridiagonality is restored. In this new approach the loss of orthogonality is not ignored but taken into account. So all the matrix elements and all overlap elements are calculated. 
This does not lead to a triangular effective Hamiltonian, but to a generalized eigenvalue problem:

$$
\begin{array}{ll}
H^{\mathrm{eff}}\left|E_{i}^{\mathrm{eff}}\right\rangle=E_{i}^{\mathrm{eff}} W\left|E_{i}^{\mathrm{eff}}\right\rangle \\
\text { with } \quad H_{i j}^{\mathrm{eff}}=\left\langle f_{i}|H| f_{j}\right\rangle \\
& W_{i j}=\left\langle f_{i} \mid f_{j}\right\rangle .
\end{array}
$$

The price to pay is that all overlap matrix elements $W_{i j}$ and matrix elements $H_{i j}^{\text {eff }}$ have to be calculated, ${ }^{17}$ i.e., all Lanczos vectors generated during the calculation have to be stored. In the standard Lanczos without MPS this is usually the bottleneck as memory is the main constraint and therefore such an algorithm is not useful there. With Lanczos vectors represented by MPS the required memory to store one vector is much smaller and therefore this approach to get rid off the 'ghosts' is manageable. The full solution of the generalized eigenvalue problem is not computationally expensive at all as the dimension of the effective Hamiltonian only scales with the number of iterations. ${ }^{18}$

\section{Correction vector}

Shortly after Hallberg's original proposal, Ramasesha et al. $\left[\mathrm{RPK}^{+} 96\right]$ and later Kühner and White [KW99] introduced the correction vector method. The basic idea is to calculate the vector

$$
\left|\Psi_{\text {corr }}\left(\omega, \eta, A^{\dagger}\right)\right\rangle=\frac{1}{\omega-\left(H-E_{0}\right)+\mathrm{i} \eta} A^{\dagger}\left|E_{0}\right\rangle
$$

The spectral function can then be calculated by computing the scalar product, see Eqn. (2.16):

$$
\rho_{A}(\omega>0)=-\lim _{\eta \rightarrow 0} \frac{1}{\pi} \operatorname{Im}\left\langle E_{0}|A| \Psi_{\text {corr }}\left(\omega, \eta, A^{\dagger}\right)\right\rangle
$$

Within the MPS formulation Weichselbaum et al. [WVS $\left.{ }^{+} 09\right]$ noted that the correction vector can be calculated variationally by minimizing the norm:

$$
\|\left|\Psi_{c o r r}\left(\omega, \eta, A^{\dagger}\right)\right\rangle-\frac{1}{\omega-\left(H-E_{0}\right)+i \eta} A^{\dagger}\left|E_{0}\right\rangle \| .
$$

However, the minimization becomes ill-conditioned as $\eta \rightarrow 0$, with conditioning deteriorating quadratically in $\eta$ [WVS $\left.{ }^{+} 09\right]$. Therefore they suggested a better way to calculate the correction vector. Again a local optimization scheme for the $M^{\sigma_{k}}$ matrix in the spirit of the DMRG is applied by directly solving

$\frac{\delta}{\delta M^{\sigma_{k}}}\left[\left\langle\Psi_{\text {corr }}\left(\omega, \eta, A^{\dagger}\right)\left|\left(\omega-\left(H-E_{0}\right)+\mathrm{i} \eta\right)\right| \Psi_{\mathrm{corr}}\left(\omega, \eta, \mathrm{A}^{\dagger}\right)\right\rangle-\left\langle\Psi_{\mathrm{corr}}\left(\omega, \eta, \mathrm{A}^{\dagger}\right)\left|\mathrm{A}^{\dagger}\right| \mathrm{E}_{0}\right\rangle\right]=0$

\footnotetext{
${ }^{17}$ The calculation of the overlap elements and the matrix elements can be done in parallel. It should be remarked that with the adaptive DMRG method one cannot calculate these overlap elements as not all the Lanczos vectors are in the same basis.

${ }^{18}$ The number of iterations is usually between $100-300$.
} 
using a non-hermitian equation solver, e.g. some conjugate gradient variant at each step. This leads to better conditioning deteriorating only linearly. In the original DMRG the correction vector can be calculated most efficiently by an extension to the DMRG called Dynamic DMRG proposed by Jeckelmann [Jec02]. There at every step in the DMRG algorithm a functional has to be solved with a conjugate gradient method in a similar way as shown above for the MPS.

The advantages of the correction vector method is that it achieves good precision at every frequency point. However, the broadening is inherent and for every different broadening a new calculation has to be done. The calculation of the correction vector due to the need of solving a non-linear equation set is usually limited to small matrix dimensions for the correction vector. For decreasing broadening the calculations become more and more difficult. The overall computational costs for a high resolution spectral function are very high as for every frequency point a new correction vector has to be calculated. Due to the needed large broadening the issue of finite-size scaling as already mentioned in the classical Lanczos method, see Sec. 3.1.2, is a non-trivial problem.

However, the correction vector method has established itself as the standard method for calculating spectral functions in the frequency domain and has been applied to many model systems, see e.g. Refs. [PRSB99, KWM00, Jec02, Jec03, BGJ04, NJ04, RUA04, RU05, FKMS07, BJ07, SSMS09, $\mathrm{WVS}^{+}$09, Pet11, GSR $\left.{ }^{+} 11\right]$.

\section{Fourier transform of time-dependent DMRG data}

Fourier-transforming time-dependent DMRG data offers another approach to dynamic spectral functions. The time evolution of the state $|\Psi(t)\rangle=e^{-i H t} A^{\dagger}\left|E_{0}\right\rangle$ is usually calculated via a Trotter decomposition of the time evolution operator $e^{-i H t}$ [Vid03, Vid04, WF04, VGRC04, Sch05a]. It has found many successful implementations, see e.g. Refs. [WF04, SK05, GR06, $\mathrm{PSC}^{+}$06, PLAH08, PWA09, BSW09, BKL ${ }^{+}$11, KBM12]. However, accessing long time scales, to obtain good frequency resolution, is limited by a rapid increase of entanglement and the resulting increasing demand of computational resources. The problematic $\eta$-broadening is also a feature of spectral functions obtained from time-dependent DMRG where the finite time-range of simulations is damped out by an artificial damping factor $e^{-\eta t}$ which leads to Lorentzian broadening in frequency space.

An attempt to extrapolate the results to longer times to get a higher resolution in the frequency domain was suggested by Pereira et al. [PWA08, PWA09] under the name 'linear prediction'. In a recent work Wang et al. [WZDX10] calculate spectral functions for the SIAM by this approach. They extrapolated the finite time data to infinity and by this could get high resolution frequency spectral functions. However, the extrapolation introduces an additional (uncontrolled) error and has to be handled carefully. 


\section{Chebyshev polynomials}

Very recently, dynamic spectral functions were also calculated using an expansion of the spectral functions in terms of Chebyshev polynomials $\left[\mathrm{HWM}^{+} 11\right]$. Like in the Lanczos approach vectors from the Krylov space are recursively created:

$$
\left|t_{0}\right\rangle=A^{\dagger}\left|E_{0}\right\rangle,\left|t_{1}\right\rangle=H\left|t_{0}\right\rangle,\left|t_{n}\right\rangle=2 H\left|t_{n-1}\right\rangle-\left|t_{n-2}\right\rangle .
$$

The expansion parameters (moments) of the Chebyshev expansion are then given by scalar products of these vectors:

$$
\mu_{n+n^{\prime}}=2\left\langle t_{n} \mid t_{n^{\prime}}\right\rangle-\mu_{n-n^{\prime}}
$$

This approach will generate a continuous spectral function, however damping, while taking a slightly different form, is also inevitable. In a review article on Green's function techniques Weisse and Fehske [WWAF06] compared the expansion in Chebyshev polynomials to the Lanczos algorithm. The Lanczos algorithm will always give a high resolution close to the extremal eigenvalues, the Chebyshev polynomials in comparison have the same precision in the whole frequency range. As the Chebyshev polynomials are a priori non-orthogonal, 'ghost' problems like in the Lanczos algorithm are not an issue. Furthermore they state that for (non-MPS) systems the Chebyshev expansion is numerical more stable. A further discussion is postponed to Sec. 5.3.

\section{Numerical renormalization group vs. matrix product states 3.3 .8}

After the developments of DMRG/MPS it was obvious to analyse whether the variational optimization (of ground states) leads also to higher accuracy for the semi-infinite chain model used in the NRG. Remember, in the NRG the SIAM is mapped on a semi-infinite chain model with exponentially decreasing hopping parameters. In particular, the rather simple truncation scheme of the NRG should be inferior to the more elaborate one of the DMRG/MPS. Weichselbaum et al. [SWvD08, $\mathrm{WVS}^{+} 09$, Wei11] could show that indeed a higher ground state precision can be achieved by the variational optimization of the semiinfinite chain model. However, the overall improvements - also for spectral functions were rather disappointing:

\footnotetext{
"For logarithmic discretization, energy scale separation is big enough that this effect is minor and for a simple single impurity problem with a focus on the Abrikosov-Kondo-Suhl resonance the ultimate improvement is very limited, as NRG is geared to describe this feature optimally. The essential point is that energy scale separation can now be abandoned altogether due to feedback, hence also logarithmic discretization, and we may choose a more fine-grained resolution of the energy band wherever it is physically suitable. This could find a variety of applications."
}

(Review Article: "The density-matrix renormalization group in the age of matrix product states", Schollwöck, 2011, [Sch11])

Abandoning the logarithmic discretization with MPS as described by Schollwöck is also a very subtle issue. If one works directly with the SIAM and constant hopping parameters $^{19}$ the non-interacting chain has to be truncated at some point. So finite-size effects are introduced to the system that are rather large. This must be contributed to

\footnotetext{
${ }^{19}$ Constant hopping parameters will lead to a semi-circular DOS of the bath for an infinite chain.
} 
the very long-range correlations between impurity spin and the spins of the conduction electrons in the Kondo regime (see Sec. 4.2.2 and Ref. [HMS ${ }^{+}$09]). In particular, spectral functions suffer from the finite-size gap and therefore are not able to resolve the Kondo resonance at $\omega=0$, see Sec. 4.2.2 and Ref. [Dar09], with the high quality as encountered with logarithmic discretization. Therefore working directly in the thermodynamic limit with the logarithmic discretization is an advantage. Discretization errors can be cured with extension like the suggested "z-averaging" by Zitko [vP09]. Therefore in this work the NRG has been used for the spectral functions of impurity systems and the DMRG/MPS has been used for real one-dimensional systems where the NRG completely fails. However, the MPS based approach might give an advantage for more complex impurity models, see Refs. [HWvD10, Pet11]. 


\section{Long range Kondo signatures}

"WHY would anyone still want to study a physical phenomenon that was discovered in the 1930s, explained in the 1960s and has been the subject of numerous reviews since the 1970s?"

(Review article: "The revival of the Kondo effect", Kouwenhoven and Glazman, 2001, [KG01])

The Kondo effect is one of the oldest and most prominent example for a quantum many body effect due to strong correlations. Traditionally it describes an increase in the resistivity with decreasing temperature in metals with a dilute concentration of single magnetic atoms. This leads to a minimum around a specific temperature, which contradicts Matthiessen's rule of a monotonous decrease for ordinary metals [AM76]. This minimum was a long standing puzzle and a first explanation using many-body perturbation theory to third order was given by Jun Kondo in the sixties [Kon64]. Later on a complete solution was given by Wilson with the renormalization group approach and the problem was considered to be solved [Wil75].

Since 1998, due to advances in nanophysics and scanning tunnelling spectroscopy (STS), the Kondo effect for single magnetic impurities is again in the focus of modern research [KG01]. In particular, the spatial correlations between impurity spin and the spins of the surrounding host electrons is under debate. This work focuses on the simulation of experimental STS data of Fe and Co atoms buried under a $\mathrm{Cu}(100)$ surface. The simulation uses a combination of band structure and NRG calculations. The excellent agreement of simulation and experiment allows to conclude for a hitherto unobserved long range Kondo signature.

In Sec. 4.1 the historic discovery of the Kondo effect and its theoretical explanation is described. Then in Sec. 4.2 the effective Hamiltonian, the single impurity Anderson model and the Kondo model, are introduced and the main properties including spectral properties are briefly summarized. In the following Sec. 4.3 the current status of the experiments on single Kondo impurities is recapitulated with special focus on STS experiments on ad-atoms and the new experiments on buried impurities are introduced. The simulation of the experimental data is described in Sec. 4.4 and the comparison to the experiment is presented in Sec. 4.5. The chapter closes with an analysis of the data with respect to the observation of a long range Kondo signature in Sec. 4.6.

The content of this chapter is published as a letter in Nature Physics [PWD $\left.{ }^{+} 11\right]$. The presented work is a collaboration with Henning Prüser, Martin Wenderoth, Alexander Weismann, Robert Peters, Thomas Pruschke and Rainer G. Ulbrich.

It may be that some parts of the following chapter resemble the manuscript of reference $\left[\mathrm{PWD}^{+} 11\right]$. Martin Wenderoth, Alexander Weismann and Rainer G. Ulbrich planned the 
experiments. Henning Prüser and Alexander Weismann carried out the experiments and the raw data analysis. On the theoretical side the simulation and effective modelling was designed by me. I did the NRG calculations, the equation of motion and the LDOS calculations. The NRG code was provided by Robert Peters and Thomas Pruschke. Alexander Weismann calculated the band-structure (LCAO) and the free Green's functions. The manuscript was written mainly by me and Henning Prüser. All authors discussed the results and commented on the manuscript.

\title{
\begin{tabular}{l|l} 
The Kondo effect & 4.1
\end{tabular}
}

\begin{abstract}
"In the Kondo effect, electrons of all wavelengths, from atomic wavelengths up to very much larger scales, all in the conduction band of a metal, interact with the magnetic moment of each impurity in the metal (see, for example, Anderson, 1970). Theorists have difficulties with these problems because they involve very many coupled degrees of freedom."
\end{abstract}

(Nobel lecture: "The renormalization group and critical phenomena", Wilson, 1983, [Wil83])

The investigations on the Kondo effect range from its discovery in the 1930s up to the present time. This section tries to give an overview of the major milestones achieved in the theoretical understanding of the effect, but due to this very long time period cannot be completely comprehensive. It is mainly based on the book of Hewson "The Kondo problem to heavy Fermions" [Hew93] and Refs. [Wil83, Ž07, Dar09].

\section{Resistivity minimum and Kondo's explanation}

The Kondo effect occurs in non-magnetic metallic systems like copper with a small number of additional magnetic impurities. Macroscopically an increase in the resistivity below a temperature $T^{*}$ can be observed, see Sec. 1.4. In 1963 Kondo [Kon64] presented a first explanation via perturbation theory. Due to the universality of this effect (by then it was discovered in $\mathrm{Cu}, \mathrm{Ag}, \mathrm{Au}, \mathrm{Mg}$, $\mathrm{Zn}$ with $\mathrm{Cr}, \mathrm{Mn}, \mathrm{Fe}, \mathrm{Mo}$, Re, Os impurities) he modelled the interaction of the magnetic impurities with the conduction electrons via an effective Hamiltonian that was previously proposed by Zener [Zen51], Kasuya [Kas56] and Yosida [Yos57b, Yos57a], see Sec. 4.2:

$$
H_{s d}=\sum_{k, \sigma} \epsilon_{k} c_{k \sigma}^{\dagger} c_{k \sigma}+J \mathbf{S s}(\mathbf{0})
$$

In this Hamiltonian the impurity is modelled by a single localized spin $\mathbf{S}$ originated in the incomplete $d$ - or $f$-shell of the magnetic impurity. The formation of the local moment is not explained within the Kondo model. However an explanation is given by the more general Anderson model, see Sec. 4.2.1. Using perturbation theory Kondo showed that in the second order Born approximation an additional logarithmic term in the temperature has to be added to the resistivity.

"... we have $\rho_{\text {spin }}=c \rho_{m}\left(1+\left(3 z J / \epsilon_{F}\right) \log T\right)$. As we have expected, this contains a singular term involving $\log T$ which increases towards low temperatures, if $J$ is negative."

(Article: "Resistance Minimum in Dilute Magnetic Alloys", Kondo, 1964, [Kon64]) 
This term therefore leads to a resistivity minimum at a certain temperature, but also to a (non-physical) divergence at $T=0$ - the so-called Kondo problem. It was Nagaoka [Nag65] shortly afterwards who used a self-consistent approach to the problem and could show that perturbation theory breaks down below a certain temperature - called the Kondo temperature $T_{K}$, explaining the failure of perturbation theory. Furthermore he could show that at this temperature a correlated many-body state at the Fermi energy appears, that is responsible for the minimum.

\footnotetext{
"The situation seems to be quite similar to the case of superconductivity, in which the perturbational treatment breaks down at the transition temperature. From the similarity between the two cases it is reasonable to expect that, in the case of dilute alloys, there appears some correlated state, or a kind of bound state, between localized spin and conduction-electron spin at low temperatures corresponding to the Cooper pair."
}

(Article: "Self-Consistent Treatment of Kondo's Effect in Dilute Alloys", Nagaoka, 1965, [Nag65])

Similar results were obtained by Abrikosov [Abr65] and Suhl [Suh65]. Furthermore they could show that below $T_{K}$ due to the formation of this correlated state the local moment of the impurity is screened by the conduction band electrons. The resonance related to this many-body state that appears in the spectral function at the Fermi-energy below $T_{K}$ is named after them. However, a solution within the full energy (temperature) range was up to this time not found.

\section{Scaling ansatz and Wilson's renormalization group}

In 1970 Anderson [And70] proposed a scaling ansatz for the Kondo model that incorporates first renormalization group ideas that were later further developed by Wilson. His idea was to subsequently reduce the bandwidth $D$ of the conduction electrons by tracing out the degrees of freedom starting at the band edges. With perturbation theory he could show that the new system with bandwidth $\mathcal{D}$ can be described by the same Hamiltonian but with different (renormalized) coupling constants $\mathcal{J}(\mathcal{D})$. In first order with a constant density of states $\rho$ he obtained the (simplified) scaling equation

$$
\frac{d \mathcal{J}(\mathcal{D})}{d \ln \mathcal{D}}=-2 \rho \mathcal{J}^{2}
$$

Here $\mathcal{J}(\mathcal{D})$ denotes the renormalized coupling constant depending on the chosen new bandwidth $\mathcal{D}$. For the original (bare) bandwidth $D$ one should get the bare coupling constants $\mathcal{J}(D)=J$. By integrating the scaling equation from $D$ to $\mathcal{D}$ and from $J$ to $\mathcal{J}$ one can finally derive a relation between old and new coupling constants

$$
\mathcal{J}(\mathcal{D})=\frac{J}{1-2 J \rho \ln \left(\frac{\mathcal{D}}{D}\right)} .
$$

The scaling equation can also be understood in the way that the physics on the energy (or temperature) scale $\mathcal{D}$ are given by the renormalized parameter $\mathcal{J}(\mathcal{D})$ and not by the bare parameter $J$. But more important Anderson concluded:

\footnotetext{
"This is why we call the procedure 'scaling laws', because what we have done is to show that a problem with one set of parameters is entirely equivalent to that with another set. This leaves us with the hope, usually fulfilled, that by following the scaling procedure out repeatedly we can eventually reach a region where a solution by some other method may be found."
}

(Article: "A poor man's derivation of scaling laws for the Kondo problem", Anderson, 1970,[And70]) 
Furthermore, starting from the scaling equation one can show that a scale invariant relation exists between the bare and renormalized parameters: the Kondo temperature. In first order it is given by:

$$
D e^{-\frac{1}{2 J \rho}}=\mathcal{D} e^{-\frac{1}{2 \mathcal{J} \rho}}=k_{B} T_{k}^{(1)} .
$$

This insight shows that all thermodynamic quantities only depend on the single energy scale $k_{B} T_{k}$. They can be written as a function that just depends on the temperature and $T_{k}$ e.g. the impurity susceptibility is given by $\chi_{\mathrm{imp}}=\frac{1}{T} F\left(\frac{T}{T_{k}}\right)$. Nevertheless, due to the use of perturbation theory, Anderson's derivation is just valid in the regime $\rho J \ll 1$. This can also be seen as for $\mathcal{D}=k_{b} T_{k}=D e^{-\frac{1}{2 J \rho}}$ the renormalized coupling $\mathcal{J}$ diverges, showing the breakdown of this ansatz at the same energy as for Nagaoka and Suhl beforehand. Taking higher orders into account gives a better estimate of the Kondo temperature

$$
\frac{d \mathcal{J} \rho}{d \ln \mathcal{D}}=-2(\rho \mathcal{J})^{2}+2(\rho \mathcal{J})^{3}+\mathcal{O}\left((\rho \mathcal{J})^{4}\right) \quad \rightarrow \quad k_{B} T_{k}^{(2)}=D|2 J \rho|^{1 / 2} e^{-\frac{1}{2 J \rho}+\mathcal{O}\left((\rho \mathcal{J})^{4}\right)} .
$$

It was then Wilson's success with his numerical renormalization group to suggest a nonperturbative renormalization ansatz that is valid on all energy scales [Wil75]. The details of this approach have been already explained in Sec. 3.2.3. Wilson's NRG gives the correct values for thermodynamic properties on all energy respectively temperature scales. For low temperatures he could validate that the impurity spin for all non-zero interactions is screened by the conduction electrons and that the system can be described by an effective non-interacting Hamiltonian. Furthermore the concept of universality is strongly linked to the renormalization group:

\footnotetext{
"Renormalization is built on the idea that the low energy physics of a system only depend on certain gross features of the high energy physics. The family of systems with the same low energy excitation spectrum constitute a "universality class" of models. We need the concept of universality, for without we would be lost, for we could not hope to capture the physics of real-world systems with our simplified Hamiltonian models. The Anderson model, is itself a renormalized Hamiltonian, notionally derived from the elimination of high energy excitations from "the" microscopic Hamiltonian."
}

(Book: "Introduction to Many Body Physics", Coleman, 2012,[Col12])

This insight is usually described with the term "Universality of the Kondo effect": The physics of Kondo systems are not strongly dependent on the band structure of the bath or the explicit form of the hybridization or crystal structure. Even for many extensions of the model as will be discussed in Sec. 4.6.1, this universality holds and physical quantities are only dependent on the single parameter $T_{k}$ and show the same low-energy physics. With Wilson's work it became now possible to calculate properties from this effective (universal) Hamiltonian with Fermi liquid theory.

\section{Fermi liquid theory and the exact Bethe ansatz solution}

The main idea of Landau's Fermi Liquid theory [Lan58] is that the low-lying excitations of an interacting system are in one-to-one correspondence with some non-interacting Fermi gas. These excitations are then named quasi-particles. Due to Wilson's solution the lowtemperature physics of the Kondo system can be understood as a local Fermi liquid with all Fermi liquid parameters accessible through a microscopic theory. This allowed Nozières [Noz74] in 1974 to calculate specific heat, spin susceptibility and resistivity for small 
temperatures. It turns out that the effective mass of the quasiparticles and their effective interactions are proportional to the Kondo temperature. Shortly afterwards, the results of Nozières were validated by a series of papers by Yosida and Yamada with their socalled microscopic Fermi liquid theory [YY70, Yam75a, Yam75b, YY75]. They could also show that a resonance in the spectral density at the Fermi energy appears in fourth order perturbation at $T=0 .{ }^{1}$ It is exactly the Kondo or Abrikosov-Suhl resonance mentioned earlier that is represented by the quasiparticle that build the many-body quantum state screening the impurity at low temperatures. Horvatic et al. [HSZ85, HSZ87] extended this ansatz to finite temperatures showing that the resonance vanishes with increasing temperature. The appearance of the Kondo resonance at the Fermi energy was later also verified by other methods e.g. extensions to the NRG by Frota and Oliveira [FO86], Sakai et al. [SSK89] and Costi and Hewson [CH90], the variational wavefunction method by Gunnarsson and Schönhammer [Sch76, GS85, GS83b, GS83a], the non-crossing approximation (NCA) [PG89] and Quantum Monte Carlo (QMC) [SGSJ90].

On a different route Andrei and Wiegmann extended the Bethe ansatz [Bet31] in the beginning of the 1980s to give an analytical solution for the Kondo model [And80, Wie81, WT83, TW83]. Nevertheless it still remains a challenge to calculate correlation functions and dynamic quantities from the exact solution. These last theoretical investigations pretty much stated the end of the Kondo problem that from then on was considered as solved. Theoreticians from then on focused on more advanced topics like multi impurity problems [JKmW81, JV87, JV89] and multi-orbital impurities [SSK90, IFT98]. Due to new experimental techniques - especially scanning tunnelling spectroscopy - that allows to probe single impurities, interest in single impurity systems grew again starting in 1999 . This so-called "Revival of the Kondo effect" [KG01] will be addressed in Sec. 4.3.

\title{
The single impurity Anderson model and the Kondo model
}

\begin{abstract}
"While the schematic character of the model should not be ignored, we feel that nonetheless it contains the essential physics of the phenomena for such solutions as $\mathrm{Mn}, \mathrm{Fe}$, or other iron group elements in $\mathrm{Cu}, \mathrm{Ag}$, and Au."
\end{abstract}

(Article: "Localized Magnetic States in Metals", Anderson, 1961,[And61])

In order to include the strong local Coulomb interactions originating from the incomplete $d$ - and $f$-shells of the magnetic atoms (impurities) in the system, one uses effective Hamiltonians that just concentrate on the essential physics. In this work the single impurity Anderson model (SIAM) [And61, And66] is used and described in detail.

\section{Definition $\quad 4.2 .1$}

At first the conduction electrons that interact with the single impurity of the host metal are modelled. In this work the host material is copper. Copper is a transition metal

\footnotetext{
${ }^{1}$ This perturbation theory gives just qualitative correct results as for strong interactions the form of the resonance is not exact.
} 
with a filled $3 d$-shell with electron configuration $[A r] 3 d^{10} 4 s^{1}$. For pure crystals of this element the $4 s$ and $3 d$ bands overlap and the Fermi energy lies within this hybridized band [AM76]. In order to build an effective model for the physics around the Fermi energy the host material is generically described by a single-band model

$$
H_{\text {cond }}=\sum_{k \sigma} \epsilon(k) c_{k \sigma}^{\dagger} c_{k \sigma} \text {. }
$$

$c_{k \sigma}^{\dagger}$ and $c_{k \sigma}$ are the usual creation and annihilation operators with spin $\sigma$, momentum $k$ and $\epsilon(k)$ is the dispersion relation. In this work the dispersion relation has to be calculated for the hybridized $s$ - $d$ band of copper.

The next step is to model the impurity. In this work Fe and Co magnetic impurities are used which are $3 d$ transition metals with an electron configuration $[A r] 3 d^{6} 4 s^{2}$ resp. $[A r] 3 d^{7} 4 s^{2}$ [MM03]. Hence, there are just 6 respectively 7 electrons from the 10 possible electrons in the five $3 d$-orbitals. For isolated Fe and Co atoms the $3 d$ orbitals would be filled according to Hund's rules [MM03]. Neglecting spin-orbit coupling this would lead to a net magnetic moment of $S_{F e}=2$ and $S_{C o}=3 / 2$. However, in the copper crystal due to crystal field splitting the degeneracy of the five $3 d$-orbitals of $\mathrm{Co}$ and $\mathrm{Fe}$ is lifted [AM76, MM03]. For the case of $\mathrm{Co}$ and $\mathrm{Fe}$ in $\mathrm{Cu}$ one usually gets threefolddegenerate $t_{3 g}$ orbitals with lower energy and twofold-degenerate $e_{g}$ orbitals with higher energy [AM76, MM03]. ${ }^{2}$ For very small energy splitting Hund's rule are still valid and a net magnetic moment of $S_{F e}^{\text {high }}=2$ and $S_{C o}^{\text {high }}=3 / 2$ will develop - called "high-spin complex" [MM03]. For very large energy splitting the $t_{3 g}$ orbitals will be occupied at first leading to a net magnetic moment of $S_{F e}^{\text {low }}=0$ and $S_{C o}^{\text {low }}=1 / 2$ - called "low-spin complex" [MM03]. However, the energy of the (highly localized) lower $t_{3 g}$ orbitals will be shifted upwards due to the double occupancy. Therefore, the electron occupation of the orbitals is highly dependent on the ratio between energy splitting and the energy increase due to double occupancy. Therefore intermediate occupancies are also likely to occur [MM03]. In order to setup a generic model Anderson suggested to model the five $3 d$-orbitals by a single level with energy $\epsilon_{d}$ which can be just doubly occupied [And61]. For single occupancy this leads to a spin $S=1 / 2$. Therefore roughly speaking, this choice is in the spirit of the formation of a "low-spin complex". Due to the strong localization double occupancy is penalized by a local Coulomb interaction $U$. For further discussions on this effective model, see Sec. 4.6.1:

$$
H_{\text {Imp }}=\sum_{\sigma} \epsilon_{d} c_{d \sigma}^{\dagger} c_{d \sigma}+U n_{d \uparrow} n_{d \downarrow}
$$

$c_{d \sigma}^{\dagger}$ and $c_{d \sigma}$ are the creation and annihilation operators of an electron in the effective $d$ state. In a final step the scattering processes between conduction band electrons in the host metal and the impurity is included. There is a finite probability for the conduction band electrons to occupy a state of the $d$-orbital of the magnetic impurity modelled by the single level $\epsilon_{d}$. This hybridization $V_{k}$ is (quasi-)momentum dependent:

$$
H_{\mathrm{Hyb}}=\sum_{k, \sigma}\left(V_{k} c_{d \sigma}^{\dagger} c_{k \sigma}+V_{k}^{*} c_{k \sigma}^{\dagger} c_{d \sigma}\right) .
$$

\footnotetext{
${ }^{2}$ The Jahn-Teller effect will give a further energy splitting in the $t_{3 g}$ orbitals and $e_{g}$ orbitals leading to a complete loss of the degeneracy [AM76, MM03].
} 
The total effective Hamiltonian is called the full interacting SIAM:

$$
\begin{aligned}
H_{\mathrm{SIAM}} & =H_{\mathrm{cond}}+H_{\mathrm{Imp}}+H_{\mathrm{Hyb}}+H_{\mathrm{Coulomb}} \\
& =\sum_{k, \sigma} \epsilon_{k} c_{k \sigma}^{\dagger} c_{k \sigma}+\sum_{\sigma} \epsilon_{d} c_{d \sigma}^{\dagger} c_{d \sigma}+\sum_{k, \sigma}\left(V_{k} c_{d \sigma}^{\dagger} c_{k \sigma}+V_{k}^{*} c_{k \sigma}^{\dagger} c_{d \sigma}\right)+U n_{d \uparrow} n_{d \downarrow} .
\end{aligned}
$$

The model and the validity of the approximation are further discussed in Sec. 4.6.1. In the following sections the calculations are restricted to the symmetric case. This implies halffilling and a conduction band, given by $\epsilon_{k}$, that is symmetric around the Fermi energy. In a special interaction regime $\left|\epsilon_{d}+U\right|,\left|\epsilon_{d}\right| \gg \Gamma \pi|V|^{2} \rho_{0}\left(E_{F}\right)$, the Kondo regime, the SIAM can be transformed via a Schrieffer-Wolff-Transformation [SW66] to the Kondo model:

$$
\begin{aligned}
H_{s d} & =\sum_{k, \sigma} \epsilon_{k} c_{k \sigma}^{\dagger} c_{k \sigma}+\sum_{k, k^{\prime}} J_{k k^{\prime}}\left(S^{+} c_{k \downarrow}^{\dagger} c_{k^{\prime} \uparrow}+S^{-} c_{k \uparrow}^{\dagger} c_{k^{\prime} \downarrow}+S_{z}\left(c_{k \uparrow}^{\dagger} c_{k^{\prime} \uparrow}-c_{k \downarrow}^{\dagger} c_{k^{\prime} \downarrow}\right)\right) \\
& =\sum_{k, \sigma} \epsilon_{k} c_{k \sigma}^{\dagger} c_{k \sigma}+J \operatorname{Ss}(\mathbf{0}) .
\end{aligned}
$$

In the Kondo model the Pauli exclusion principle and the Coulomb interaction are expressed by the interaction of the conduction electrons $\left(c_{k}, c_{k^{\prime}}\right)$ with a localized spin $\left(S^{z}, S^{+}, S^{-}\right.$spin-1/2-operators) and the coupling is given by the $J_{k k^{\prime}}$. In the second line the interaction is transformed to real space $^{3}$ and $\mathbf{s}(\mathbf{0})$ gives the electron spin-density at the impurity position. This model was used by J. Kondo to calculate in third-order perturbation theory the experimentally observed resistivity minimum as mentioned in Sec. 4.1.

$$
\begin{array}{l|l}
\text { Properties } & 4.2 .2
\end{array}
$$

Energy scales and Kondo temperature

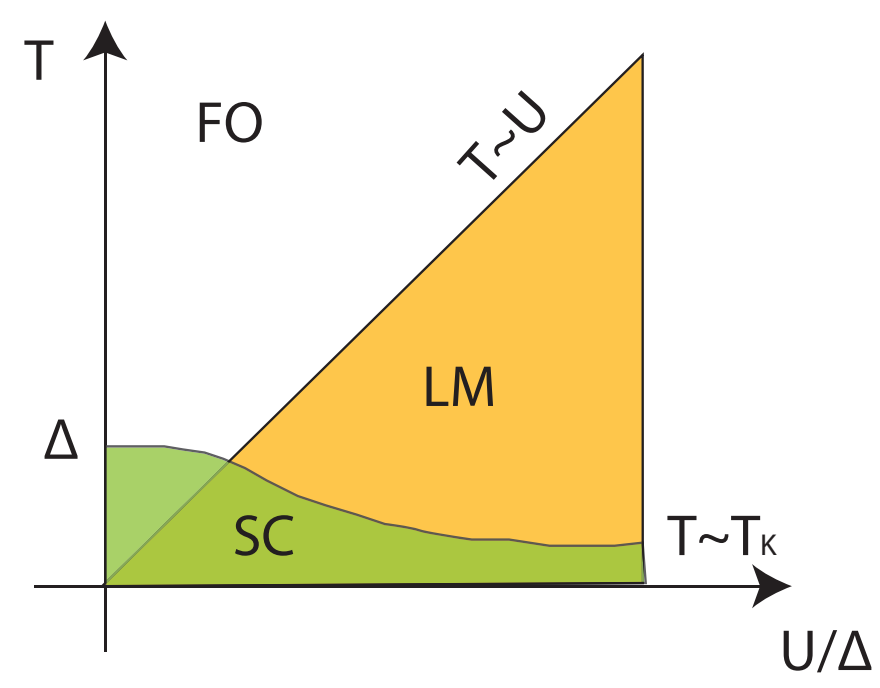

Figure 4.1: The schematic phase diagram of the symmetric SIAM on base of Ref. [Col12]. Three different regimes can be identified: FO: Free orbital regime, LM: Local moment regime and SC: Strong coupling regime.

\footnotetext{
${ }^{3}$ This mapping requires an isotropic coupling $J_{k k^{\prime}}$.
} 

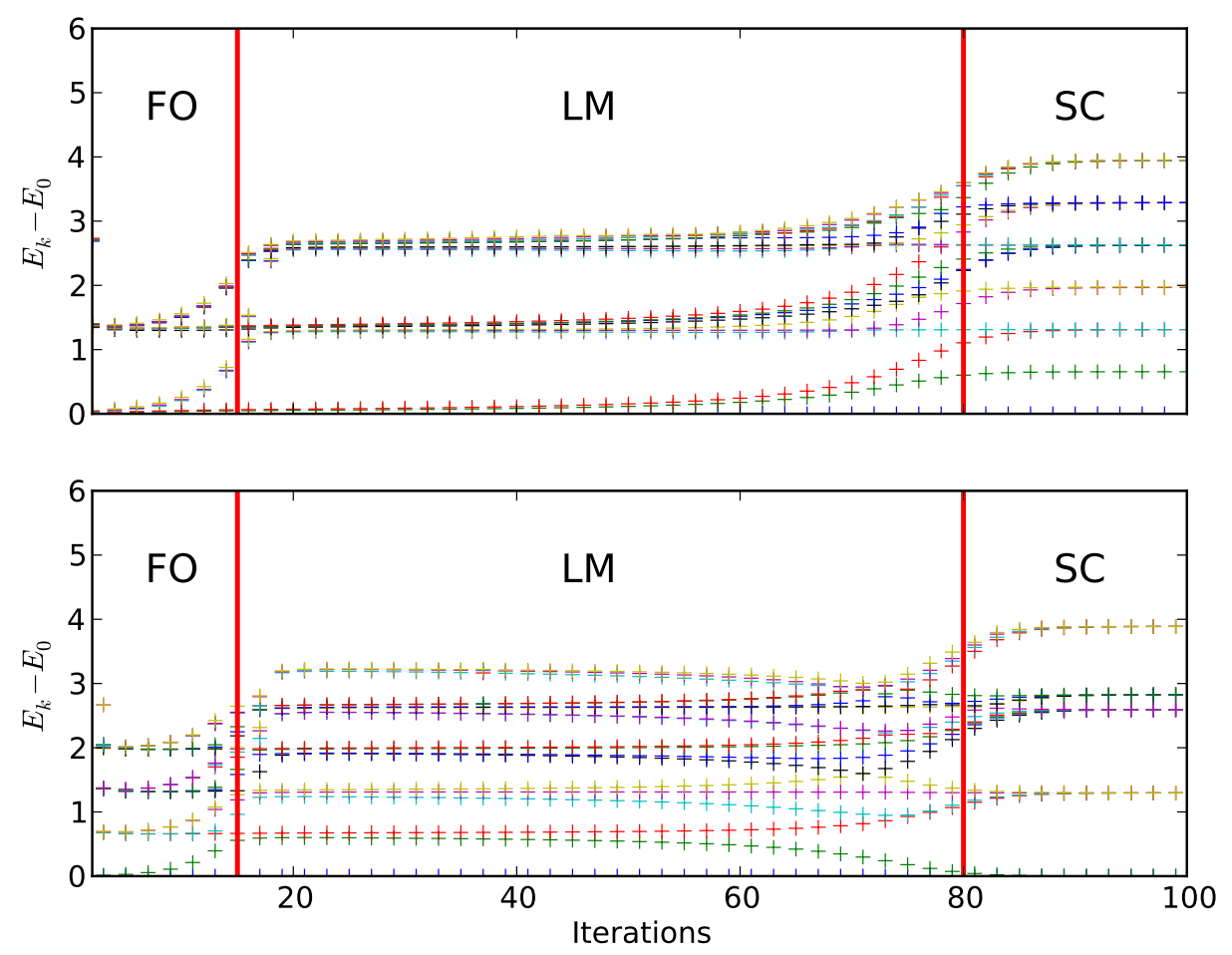

Figure 4.2: An energy flow for the symmetric SIAM with $U=0.01, \Gamma=0.0002, \Lambda=2.0$ is shown as an example. The upper panel shows the even iterations and the lower panel the odd iterations. The three energy regimes: the free orbital (FO) fixed point, local-moment (LM) fixed point and the strong-coupling (SC) fixed point are clearly recognizable. The vertical lines separating them are just a guide to the eye. See also Ref. [vP09]. (zi)

In Fig. 4.1 the phase diagram of the symmetric SIAM is shown, see also Ref. [Col12]. ${ }^{4}$ For high temperatures the system is in the so-called free orbital regime (FO). The electrons in the d-state are decoupled and the conduction band electrons can be described by a Fermi liquid. The system is dominated by thermal fluctuations. Lowering the temperature the electron in the effective d-orbital may become localized and a local moment develops. The occurrence of this local moment (LM) phase is related to the hybridization or more precisely to the ration of $U / \Delta$. For small temperatures the system will always end up in the strong coupling regime (SC), where the local moment of the impurity is screened by the conduction band electrons. However as no symmetry breaking occurs in the system, the different regimes are not separated by phase transitions, but by cross-overs.

The different energy regimes can be observed very nicely in the energy flow of the SIAM under the NRG transformation. An extensive analysis by Krishnamurthy et al. [KmWW80b, KmWW80a] showed that the energy flow for small iterations is close to a first fixed point which is named accordingly the free orbital (FO) fixed point $(U, V=0)$

\footnotetext{
${ }^{4}$ In the caption of the figures the used implementation of the NRG is denoted by the abbreviations (zi) for Žitko and (pe) for Peters.
} 


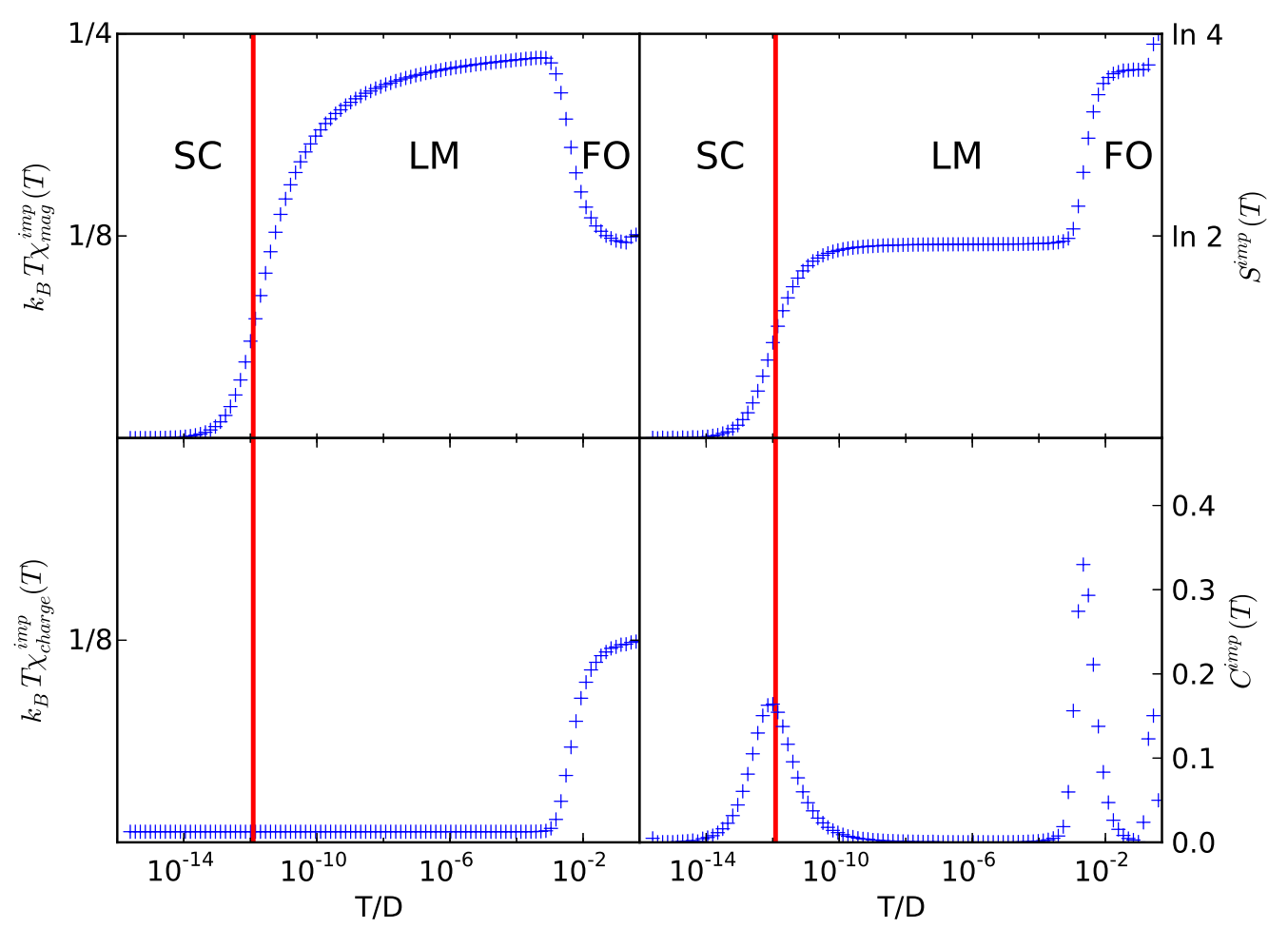

Figure 4.3: Thermodynamic static quantities for the symmetric SIAM with $U=0.01, \Gamma=$ $0.0002, \Lambda=2.0$. The red line denotes the Kondo temperature as defined in Eqn. (4.14). See also Ref. [vP09]. (zi)

[BCP08]. This fixed point is unstable and for intermediate iterations there is a rapid crossover to the local-moment (LM) fixed point. This fixed point is still unstable describing a just weakly coupled impurity spin to the conduction electrons. For larger iterations a cross-over to the strong-coupling (SC) fixed point occurs. This fixed point is stable and reached for all values of $U \neq 0$. Further RG analysis, in the spirit of Sec. 3.2.2, showed that there is a relevant perturbation that drives the system away from the FO fixed point. The LM fixed point just has a marginal perturbation which slowly drives the system away from it. The strong coupling fixed point has only irrelevant perturbations explaining its stability. In Fig. 4.2 an energy flow of the NRG is shown as an example which nicely demonstrates the different energy regimes. See also Ref. [vP09]. For a better understanding of the physics in these three regimes the analysis of thermodynamic quantities is very helpful. In Fig. 4.3 some thermodynamic quantities are shown which were defined in Sec. 3.2.3. This analysis is based on Ref. [vP09]:

- Free orbital (FO) regime:

This is the high temperature regime. All four impurities states $|0\rangle,|\uparrow\rangle,|\downarrow\rangle$ and $|\uparrow \downarrow\rangle$ have equal probability. Therefore the impurity entropy is $S^{\mathrm{imp}}=\ln 4$. Strong charge and spin fluctuations are still dominant, which can be seen in the corresponding susceptibilities. The system can be described by a Fermi liquid. 
- Local moment (LM) regime:

In this regime the impurity can be effectively modelled by a local moment (spin). The charge degrees of freedom are essentially frozen out and the impurity is only in the two states $|\uparrow\rangle,|\downarrow\rangle$. This can be directly seen in the impurity entropy that reduces to $S^{\mathrm{imp}}=\ln 2$. Another hint is given by the charge fluctuations (see charge susceptibility) which drops to zero and the increase of the spin fluctuations (see spin susceptibility). The system behaves like the Kondo model for these temperatures and below.

- Strong coupling $(S C)$ regime:

Below the Kondo temperature $T_{K}$ the impurity spin is completely screened by the conduction electrons. A many-body singlet state between impurity spin and conduction electron spins is formed. Therefore the impurity entropy drops to $S^{\mathrm{imp}}=\ln 1=0$ and no spin fluctuations are possible anymore. The system again can be described as a Fermi liquid. However, the quasiparticles are not the original bandstates anymore. Moreover, in comparison to the Fermi liquid phase in the FO regime the number of quasiparticles has increased by one ("Large Fermi surface").

The Kondo temperature $T_{K}$ can be calculated from the SIAM parameters: interaction U, the on-site energy $\epsilon_{d}$, bandwidth $D$ and the Anderson width $\Delta$ in the limit $\left|\epsilon_{d}+U\right|,\left|\epsilon_{d}\right| \gg$ $\Delta$, see Ref. [Hew93]:

$$
T_{K} \propto D \sqrt{\frac{\Delta U}{\left|\epsilon_{d}\right|\left|\epsilon_{d}+U\right|}} \exp \left(-\pi \frac{\left|\epsilon_{d}\right|\left|\epsilon_{d}+U\right|}{2 \Delta U}\right) .
$$

Following Ref. [Ž07] the proportionality constant is given for a symmetric model by: ${ }^{5}$

$$
T_{K}=0.182 U \sqrt{\frac{8 \Gamma}{\pi U}} \exp \left(-\frac{\pi U}{8 \Gamma}\right) .
$$

Using Eqn. (4.14), the red vertical line in Fig. 4.3 is positioned at the Kondo temperature - directly at the cross-over between LM and SC regime.

\section{Kondo resonance}

Applying microscopic Fermi Liquid theory [Lan58, YY70, Yam75a, Yam75b, YY75] to the SIAM, one can conclude that the quasi-particle density of states has a resonance at the Fermi level. If the single impurity level $\epsilon_{d}$ is well below the Fermi level one can see that the resonance is very distinct and a true many-body effect. It is referred to as the Kondo resonance or Abrikosov-Suhl resonance. In temperature dependent spectral functions this resonance will just evolve below a temperature of $T_{k}$ [HSZ85, HSZ87] - showing its relation to the many-body singlet state. NRG calculations [FO86, SSK89, CH90] could verify this prediction from the Fermi Liquid theory as one can see a sharp resonance at the Fermi energy in the spectral densities of the one electron Green's function $G_{d d}(\omega)$.

\footnotetext{
${ }^{5}$ Here the parameter $\Gamma=\pi \Delta\left(\epsilon_{F}\right) \rho^{0}\left(\epsilon_{F}\right)$ is used that is given by the Anderson width $\Delta(\omega)$ (See Eqn. (3.18)).
} 


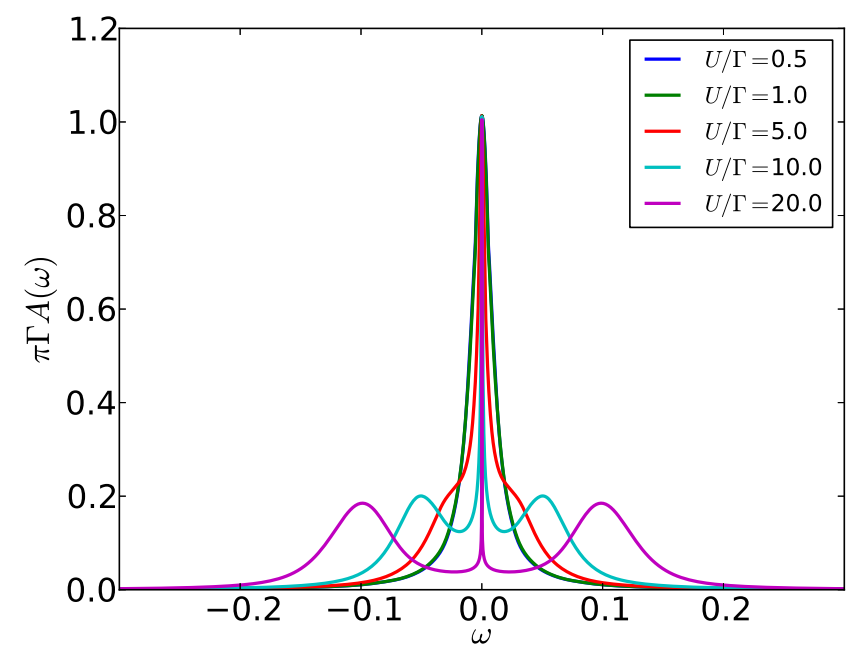

Figure 4.4: The spectral function of the SIAM for different values of the interaction $\mathrm{U}$ at $T=0$. The emergence of the Kondo resonance from the Lorentzian is nicely recognizable. For the calculation 8 z-values were used with $\Lambda=4$. (zi)

In Fig. 4.4 Kondo resonances in the spectral function $A(\omega)=-\frac{1}{\pi} G_{d d}(\omega)$ for different values of the interaction are shown. For $U=0$ the resonance is a Lorentzian. With increasing interaction $U$ the resonance gets narrower, but due to the Friedel sum rule [Hew93] it is pinned to the value of $\frac{1}{\pi \Gamma}$. For large $U$ most of the spectral weight is shifted into the Hubbard peaks that emerge at higher frequencies. In Fig. 4.5 the spectral function is shown for different values of $U / \Gamma$, but with an energy scale in terms of the Kondo temperature. One can clearly see that for values $U / \Gamma \gtrsim 3$ all the curves collapse on a universal curve showing the expected universality of the Kondo effect. This energy regime at $T=0$ for $U / \Gamma \gtrsim 3$ is sometimes called the Kondo regime. For experimentalists the Kondo resonance offers a way to extract the Kondo temperature. This is usually done via the half-width at half-maximum (HWHM) $\Delta_{K}$. In the crudest approximation the Kondo resonance is modelled via a Lorentzian $\rho_{L}(\omega) \propto \frac{\Gamma_{L}}{\Gamma_{L}^{2}+\omega^{2}}$. This is only a valid approximation in the asymptotic $\omega \rightarrow 0$ region [vP09, BGLP00] or as denoted for $U=0$. The HWHM is then directly given via $\Delta_{K}=\Gamma_{L}$. A better line shape for $U \neq 0$ gives a

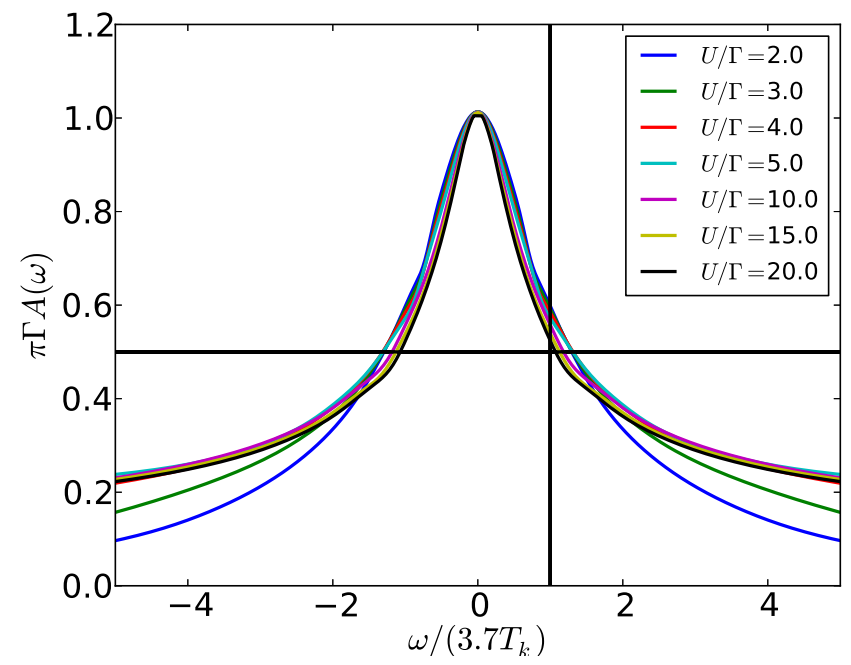

Figure 4.5: The spectral function of the SIAM for different values of the interaction $\mathrm{U}$ at $T=0$ with an energy scale divided by $3.7 T_{k}$. One can nicely see the collapse of the curves to an universal curve for $U / \Gamma \gtrsim 3$. The HWHM is denoted by the black lines. For the calculation 8 z-values were used with $\Lambda=4$. (zi) 
phenomenological form found by Frota et al. [FO86, Fro92]:

$$
\rho_{F}(\omega)=\operatorname{Im}\left[\frac{1}{\mathrm{i}} \sqrt{\frac{\mathrm{i} \Gamma_{F}}{i \Gamma_{F}+\omega}}\right] .
$$

A comparison between the phenomenological function found by Frota, the conventionally used Lorentzian and the resonance calculated with the NRG are shown in Fig. 4.6. One can clearly see the better modelling of the resonance by the Frota form. The HWHM of this form is given by $\Delta_{F}=2.542 \cdot \Gamma_{F}$. Therefore setting this form on the same value at the HWHM as the Kondo resonance gives $\Gamma_{F}=0.393 \cdot \Delta_{K}$. Following Ref. [vP09] the HWHM is proportional to the Kondo temperature - the universal energyscale:

$$
\Delta_{K}^{Z i t k o} \approx 3.7 \cdot T_{K}
$$

Therefore in this work the relation $\Gamma_{F}=1.455 k_{B} T_{K}$ is used for the Frota form. In Fig. 4.5 the HWHM is denoted by the black lines underlining the validity of the proportionality constant in the Kondo regime. Using this proportionality constant, one has to remember that it is only valid in the wide band limit for a constant band in the Kondo regime for a symmetric model. For the experimental system there will of course be deviations from these idealistic assumptions. Nevertheless, one can argue with the universality of the Kondo effect. But the values for the Kondo temperatures from the mean values of the resonance widths are to be interpreted with care.

Figure 4.6: Comparison of the Kondo resonance calculated with the NRG in the strong Kondo regime, the phenomenological Frota form and a Lorentzian. The energy is rescaled to the Kondo temperature. One can clearly recognize the better agreement between NRG and the Frota form. (zi)

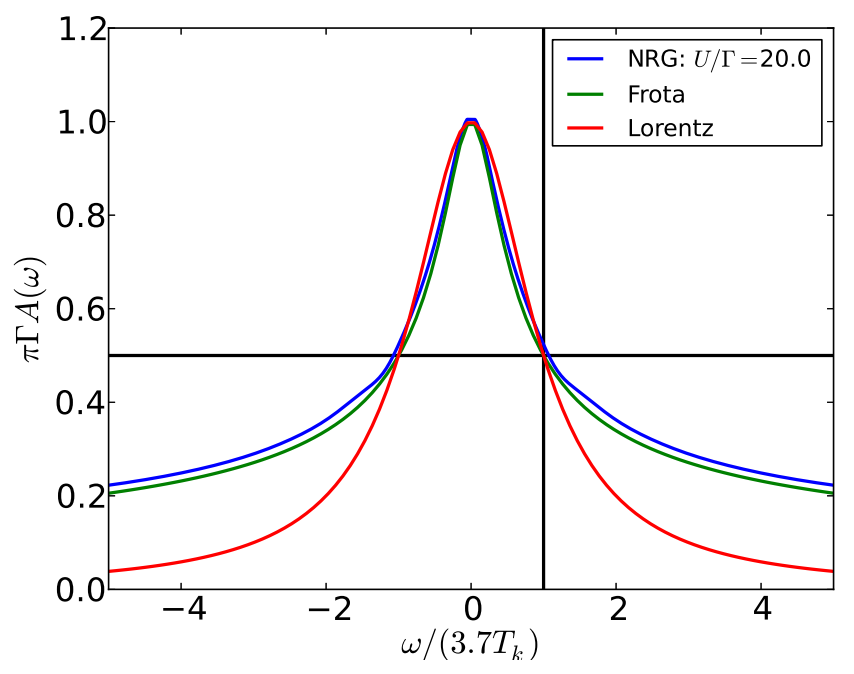

\section{Kondo cloud}

From the universality of the Kondo effect it is natural to introduce a corresponding length scale $\xi_{K}$ related to the single quantity $T_{K}$ :

$$
\xi_{K}=\hbar v_{F} /\left(k_{B} T_{K}\right)
$$

One might expect all spatial physical observables only to be dependent on $\xi_{K}$ or respectively $\xi_{K} / r$, where $r$ denotes the distance to the impurity. Simplified this length scale gives 


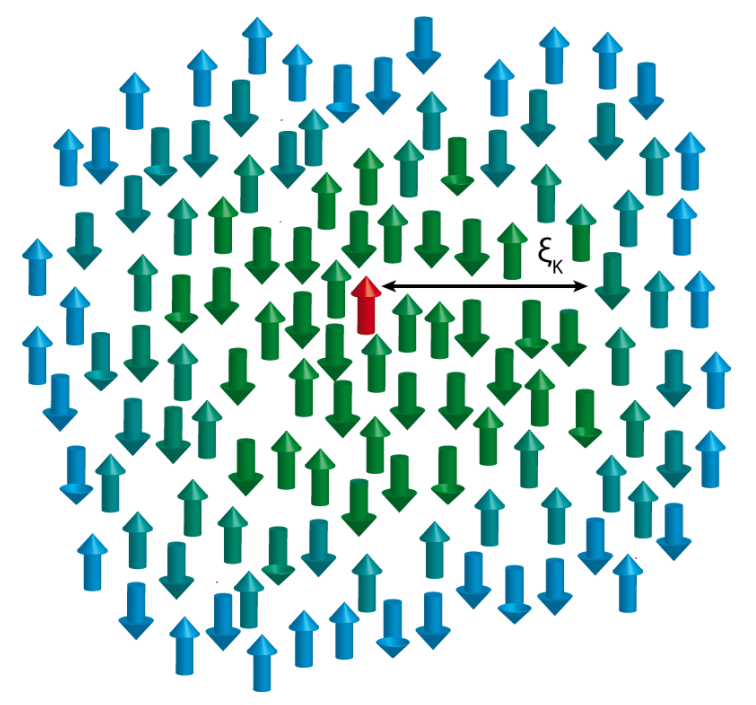

Figure 4.7: A conceptional sketch of the Kondo cloud with no lattice structure. In the strong coupling regime charge fluctuations are absent and the ground state is given by a many-particle singlet state between the impurity spin (red) and the conduction band electron spins (green-blue). The colour coding of the conduction band spins symbolizes the strength of the spin-spin-correlations between the impurity spin and the conduction band electron spin (green - strong, blue - weak). The range of these correlations is given by the universal length scale $\xi_{K}$.

the spatial extension of the conduction electrons that are needed to screen the impurity spin [BA96, BA98, Aff09, MBB11]. There are some physical observables that are known to show this spatial dependence of the parameter $\xi_{K}$, see Ref. [Aff09] for a overview. The most discussed ones are the knight shift - the magnetic polarization of the electrons as a function of distance from the impurity [SA96], the charge density [GHS87, ABS08, Ber08], the current trough a quantum dot [AS01, SA03, SA05], and static spin-spin correlation functions [GHS87, CGTR06, HKM06, Bor07, $\mathrm{HMS}^{+}$09]. Nevertheless, as of now in none of the observables one could experimentally measure the Kondo cloud [Aff09]. In Sec. 4.6.3 the simulation and experimental data are discussed if they provide an access via the charge density to the Kondo cloud.

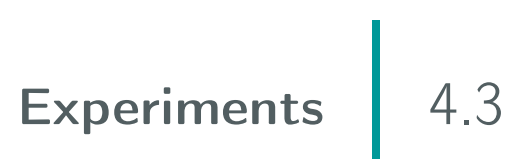

The existence of the Kondo resonance has been experimentally confirmed for dense systems with high resolution photoemission electron spectroscopy and inverse photoemission [PDSB85, $\mathrm{EHR}^{+}$07]. Due to their limited spatial resolution these measurements always probe a very large ensemble of magnetic atoms. With its capability to study local electronic properties with high spatial and energetic resolution, scanning tunnelling spectroscopy (STS) gives an unique access to individual impurities [LSBD98, MCJ ${ }^{+}$98].

\section{\begin{tabular}{l|l} 
Scanning tunnelling spectroscopy & 4.3 .1
\end{tabular}}

In a scanning tunnelling microscope (STM) [BR87] a sharp metal tip is put in close proximity to a sample surface. This close distance between tip and sample leads to an overlap between tip and sample wavefunction and thus to a non-zero probability of the transmission of electrons. A further applied voltage $V$ results in a current $I$ that is very sensitive to the tip-sample distance $h$. The constant current mode allows to record a topography $h(\mathbf{x})$ at every single point $\mathbf{x}$ of the surface. Therefore the current is held constant by adjusting the tip distance $h(\mathbf{x})$. The term scanning tunnelling spectroscopy 
(STS) is used if one records an $I(V, x)$ curve at a certain position $\mathbf{x}$ on the surface. Tersoff and Harmann [TH83, TH85] could show that with some approximations the $\frac{d I(V, x)}{d V}$-curve is proportional to the $\operatorname{LDOS}(x, \omega)$ :

$$
\frac{d I(V, x)}{d V} \propto \operatorname{LDOS}(x, \omega)
$$

See Refs. [BR87, Wie94, BR99] for further details on the experimental aspects and the theoretical data analysis.

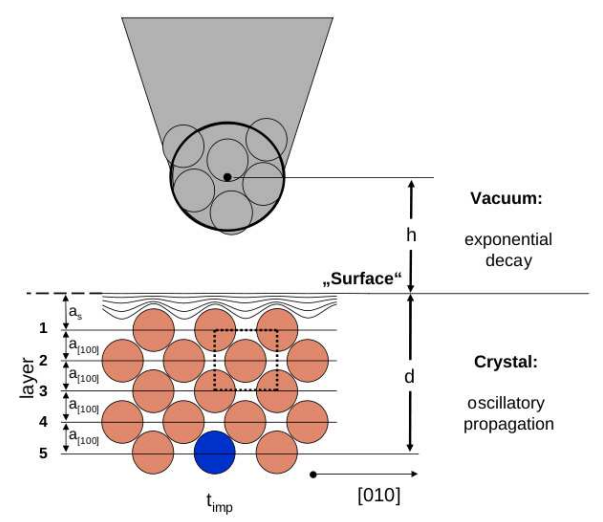

Figure 4.8: (Figure taken from Ref. [Wei08]) The schematic setup of an STM for the analysed experiments in this work. The STM tip consists only of a few atoms, which is positioned in very close proximity to the sample.

\section{\begin{tabular}{l|l} 
Kondo signatures from ad-atoms & 4.3 .2
\end{tabular}}

The main focus of modern experimental studies of single impurity Kondo systems is on the extend (range) and analysis of spatial correlations. A theoretical prediction for the local density of states (LDOS) was first provided by Újsághy et al [UKSZ00]. According to their calculations the Kondo resonance induces strong spectroscopic signatures at the Fermi energy whose line shape is oscillatory with distance to the impurity - see Sec. 4.4.3 for further details.

Since the first STS studies in 1998 [LSBD98, MCJ+98] a lot of experiments on magnetic atoms and molecules on metal surfaces were performed, all revealing Kondo fingerprints [MLE00, KSD ${ }^{+}$02, $\mathrm{QWW}^{+}$04, $\mathrm{WDS}^{+}$04, $\mathrm{ZLC}^{+}$05, IDH06, NKL ${ }^{+}$07, NKB ${ }^{+}$08]. But quantitative analysis of the surface Kondo effect is difficult due to the ongoing discussion of the influence of surface states [KSD ${ }^{+} 02$, LB04, HM07]. However, it is worth noting that all previous STS experiments have reported that the Kondo signature rapidly vanishes and no variation of the line shape occurs when the tip is moved away laterally from the impurity atom, for a review see Ref. [THS09].

The most spectacular experiment is given by a quantum mirage Kondo resonance in the second focus of an elliptical corral on $\mathrm{Cu}(111)$, where only the first focus is occupied by a Co adatom [MLE00]. By this elliptical resonator structure the electron's amplitude is amplified in the second focus and thus showing an artificial long range Kondo signature in the second focus. However, no line shape variations with distance were reported. 


\section{Kondo signatures from buried magnetic impurities $\quad 4.3 .3$}

The missing success of observing long range signatures of the Kondo effect for surface magnetic atoms motivated a study of single isolated magnetic impurities buried below the surface of a copper crystal. It has been recently shown that the anisotropy of the copper Fermi surface leads to a strong directional propagation of quasiparticles scattered from an impurity, which is called electron focusing [WWL ${ }^{+}$09], see Sec. 4.4.1. This effect gives access to individual bulk impurities in a metal that were previously assumed to be "invisible" due to charge screening.

\section{Experimental setup and data processing}

The dilute magnetic alloys were prepared on a clean $\mathrm{Cu}(100)$ single crystal by adding a small amount $(0.02 \%)$ of either Co or Fe to the topmost monolayers. The impurities are located in different depths below the surface and were investigated with a low temperature STM operating at $6 \mathrm{~K}$.

In order to obtain the LDOS $(\mathbf{x}, \omega)$ STS spectroscopic data was acquired by recording an $I(U)$ curve at every scanning point with interrupted feedback loop. Further data processing included averaging and numerical differentiation. This provides a complete map of the differential conductance $d I / d V$ as a function of lateral tip position and bias voltage on the $\mathrm{Cu}(100)$ surface. Since the tip is stabilized in constant current mode, the STS data is affected by different tip-sample distances. This artefact can be successfully removed by normalizing the STS data to a constant sample tip distance [GWS $\left.{ }^{+} 04\right]$. To extract the LDOS change, the differential conductance of the free surface $d I / d V$ is subtracted. This quantity is proportional to the change in the LDOS [WDSK08] at the lateral tip position. For further details on these experiments please see Refs. [Wei08, Pru08, PWWU12].

\section{Experimental observation}

Fig. 4.9 shows constant current topographies of Co impurities buried below a $\mathrm{Cu}(100)$ surface. The ring-like standing wave pattern can be explained by the focussing effect (see Sec. 4.4.1). At first the depth $d_{n}$ of each impurity in monolayer (ML) below the surface can be determined by analysing the topographies:

\footnotetext{
"... the observed standing wave patterns are ordered by the lateral size. The size of the standing wave pattern increases monotonically with depth of the impurity. As a verification of the obtained impurity position we use atomically resolved topographies. Since the $\mathrm{Cu}(100)$ has an fcc crystal structure and impurity atoms are located on lattice sites, the impurity contrast follows a certain pattern with respect to the host lattice. We label the surface layer as 1st monolayer. The standing wave pattern of an odd layer impurity is centred on a corrugation maximum of the surface. If the impurity is positioned in an even layer the centre is located between the corrugation maxima."
}

(Article: "Mapping Itinerant Electrons around Kondo Impurities", Prüser et al. , 2012, [PWWU12])

The observed LDOS modulation amplitudes are in a range of $6 \%$ (7 monolayer) - 13\% (3 monolayer).

Fig. 4.10 a) shows again STM topographies, but of a fifth layer Fe impurity for different bias voltages at $-50 \mathrm{mV}$ and $50 \mathrm{mV}$. The local minimum of the LDOS present 

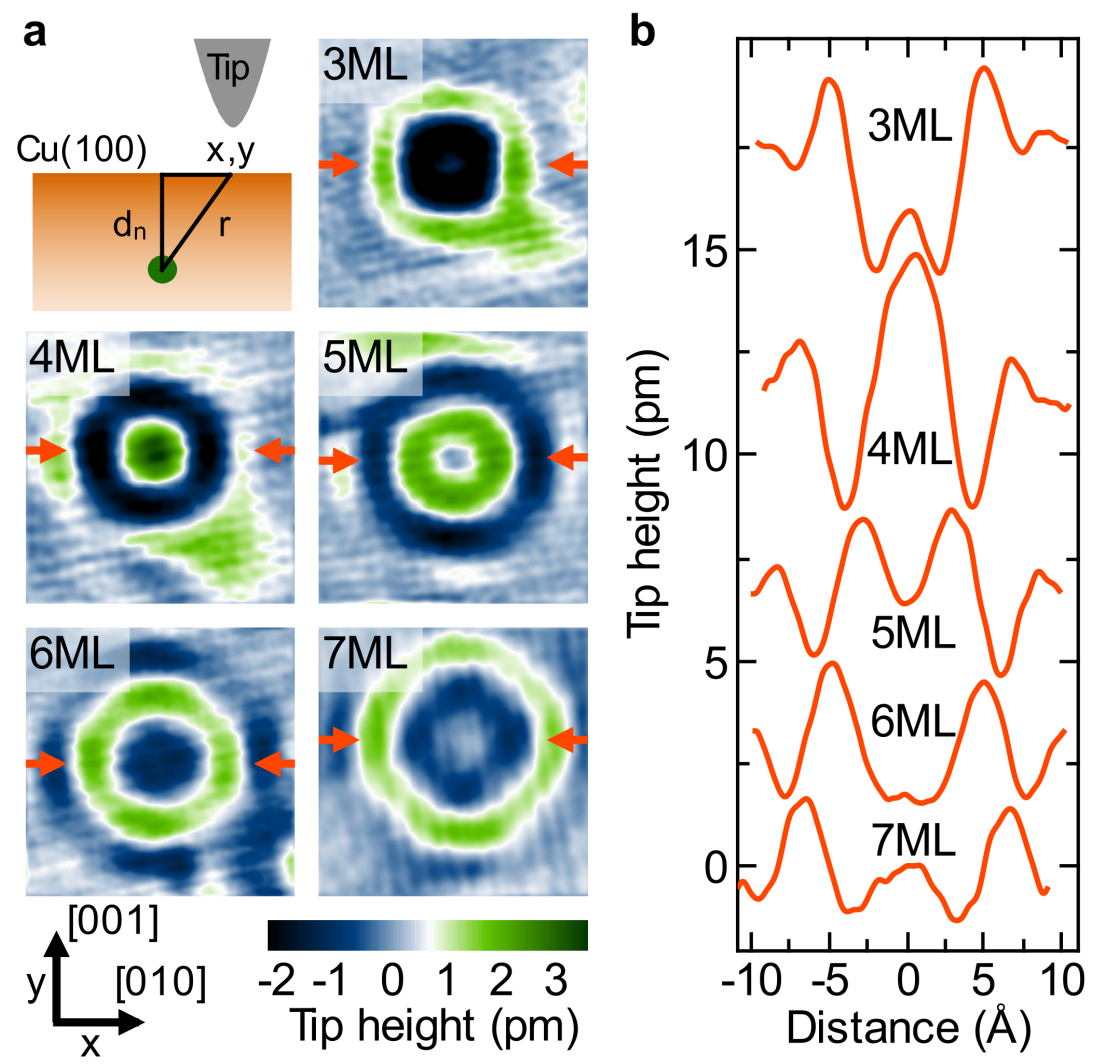

Figure 4.9: (Figure taken from Ref. [PWWU12]) a) Constant current topographies (2nm x $2 n m, V=10 m V, I=2 n A$ ) of single Co atoms buried below a $\mathrm{Cu}(100)$ surface. (b) Corresponding cross sections (shifted vertically for clarity) of each layer along the $x=[010]$ direction, marked by the arrows in (a)

in the centre of interference pattern for $V<0$ develops into a plateau like maximum for $V>0$. In a second step the LDOS modulations are analysed. In Fig. $4.10 \mathrm{~b}$ ) the differential conductance as a function of bias voltage and one spatial coordinate $y$ across the impurity pattern is shown. The crossover observed in the topographies occurs very close to zero bias. In Fig. $4.10 \mathrm{c}$ ) four spectra for different positions A-D are shown, illustrating very clearly that a single Kondo atom buried below the surface of copper induces long range spectral signatures that depend on the (lateral) distance to the impurity. A second possibility to investigate the LDOS modulations versus distance is to look at impurities situated in different depth $d$ below the surface. This and further detailed analysis of the LDOS modulations will be postponed to Sec. 4.5.

\section{\begin{tabular}{l|l} 
Simulation & 4.4
\end{tabular}}

The theoretical modelling of the measured signatures consists of two steps. At first the free electron propagation in the copper crystal is analysed. In particular, as it is known from standard electronic theory, e.g. Thomas-Fermi theory, that the buried atoms should be screened by the surrounding conduction electrons and therefore invisible. However, the anisotropic Fermi surface of copper leads to a focussing effect that was discovered by 

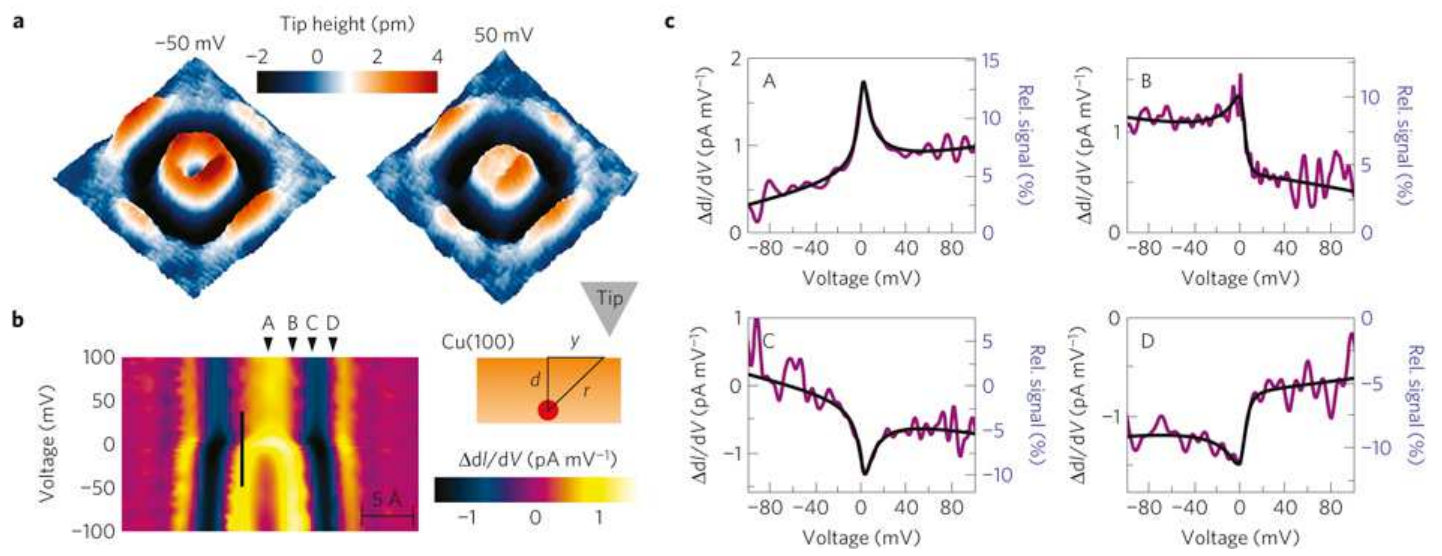

Figure 4.10: (Published in $\left[\mathrm{PWD}^{+} 11\right]$.) Variation of the LDOS with the lateral distance of the tip. a) STM constant current topography $(1.8 \mathrm{~nm} \times 1.8 \mathrm{~nm}, 2 \mathrm{nA})$ of a 5 th layer Fe impurity for different bias voltages. For negative bias voltage the LDOS directly above the impurity is reduced. By increasing the voltage the central ring contracts to a plateau like maximum and the lateral extension of the focusing pattern decreases. b) Section of the differential-conductance $\Delta d I / d V=d I / d V-d I_{0} / d V$ along the [010] direction. An energy dependent phase shift of the interference pattern can be observed. The vertical black line would resemble the phase front for a energy independent scattering phase and indicates that the overall phase shift caused by the resonance is less than $\pi$ (See Sec. 4.5). Single spectra (purple curve) for four different lateral distances (marked in b by black arrows) are depicted in c). To illustrate the relative amplitude of the Kondo signal, the data is normalized to the differential-conductance of the free surface (blue scale on the right side). The black curves are obtained by fitting the differential-conductance to a phenomenological expression (see Sec. 4.4.3).

Weismann in 2008 [Wei08, WWL ${ }^{+} 09$ ] and explains the visibility of the buried atoms at the surface.

In a second step the interaction effects - modelled by the SIAM have to be included. This is accomplished by using the Dyson equation that combines the free Green's functions of the copper crystal that show the focusing effect with the interacting Green's function of the impurity. This interacting system is then calculated with the NRG. In the end the full Green's function at the surface can be evaluated with the Dyson equation. The roadmap of the simulation is given in Fig. 4.11. The section closes with the derivation of a a phenomenological formula for the full Green's function at the surface in order to compare the theory quantitatively with the experiment

\section{Free Green's function of copper and the focussing effect $\quad 4.4 .1$}

The following short summary of the focussing effect is based on Refs. [Wei08, WWL ${ }^{+} 09$ ]. In order to explain the signatures of the impurities in the LDOS one has to regard the electron propagation in the host metal. A description by a one-particle Hamiltonian 


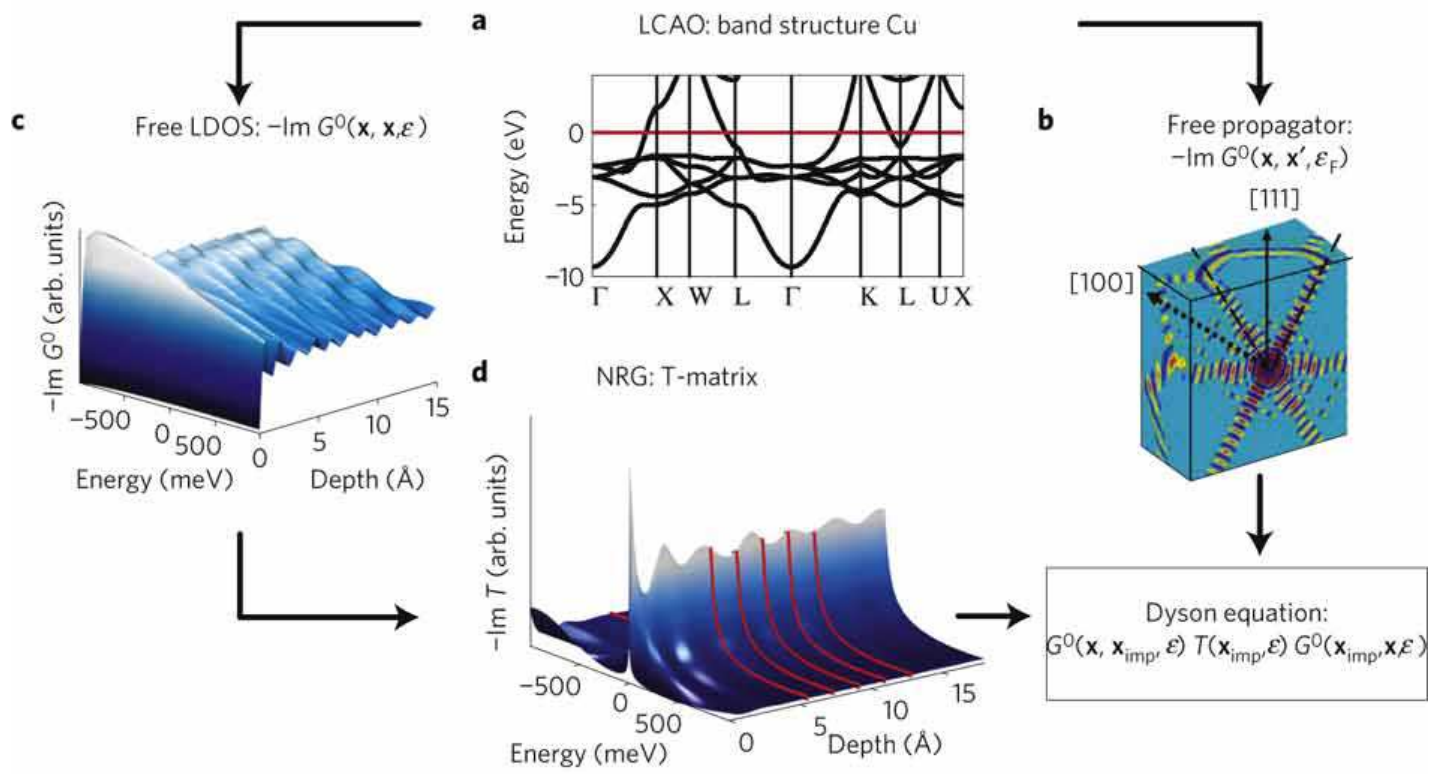

Figure 4.11: (Published in [PWD $\left.{ }^{+} 11\right]$.) Roadmap of the theoretical model: a) band structure of copper calculated using LCAO and b) the related free propagator. c) Unperturbed LDOS as function of depth including effects of the surface. d) The Kondo resonance for impurities located at different positions (pe).

is sufficient as the long range Coulomb interaction between the conduction electrons is screened, see discussion in Sec. 4.2.1:

$$
H_{\mathrm{cond}}=\sum_{k \sigma} \epsilon(k) c_{k \sigma}^{\dagger} c_{k \sigma}
$$

$c_{k \sigma}^{\dagger}$ and $c_{k \sigma}$ are the usual creation and annihilation operators with spin $\sigma$ and momentum $k$ and $\epsilon(k)$ is the dispersion relation of the host metal.

Using Bloch wavefunctions of the conduction electrons $\Psi_{k}(x)=u(k) \exp (i k x)$ the Green's function in real space reads

$$
G_{x, x^{\prime}}^{0}(\omega)=\lim _{\eta \rightarrow 0} \sum_{k} \frac{\Psi_{k}(x) \Psi_{k}^{*}\left(x^{\prime}\right)}{\omega-\epsilon(k)+i \eta}
$$

and the corresponding free LDOS is given by :

$$
\operatorname{LDOS}^{0}(x, \omega)=-\frac{1}{\pi} \operatorname{Im} G_{x, x}^{0}(\omega)=\sum_{k} \Psi_{k}(x) \Psi_{k}^{*}(x) \delta(\omega-\epsilon(k)) .
$$

Therefore the sum can be constrained to the momenta that fulfil $\epsilon(k)=\omega$ - that belong to the so called iso-energy surface $\Omega(\omega)$ - and the last equation can be written with Bloch wavefunctions:

$$
\operatorname{LDOS}^{0}(x, \omega)=\sum_{k \in \Omega(\omega)} u(k) u^{*}(k) \exp \left(i k\left(x-x^{\prime}\right)\right)
$$




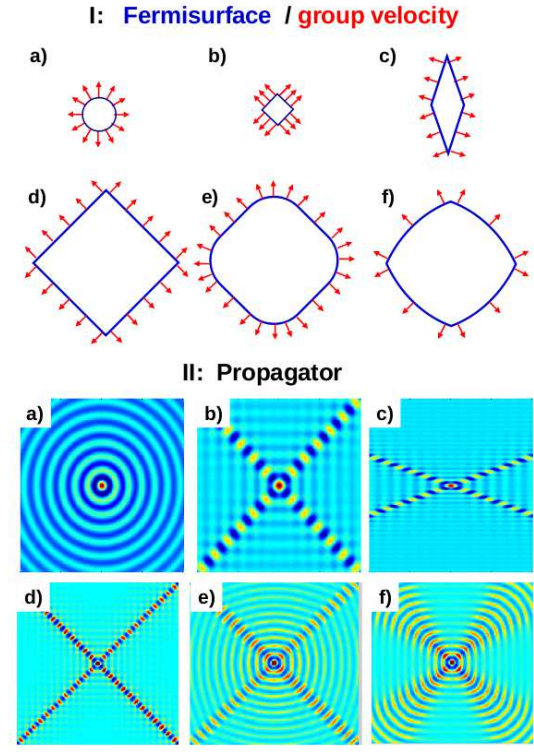

Figure 4.12: Visualization of different propagators for different Fermi surfaces in two dimensions. One can see that anisotropic Fermi surfaces can lead to electron focusing with non-decaying amplitude. One should recognize that only small deviations from a perfect anisotropic Fermi surface are sufficient to lead to electron focusing. Figure taken from the $\mathrm{PhD}$. thesis of $\mathrm{A}$. Weismann [Wei08] .

Thus the shape of the iso-energy surfaces are of great importance for the propagation of electrons. ${ }^{6}$ Concentrating on the low-energy physics that means energies around the Fermi-energy, the shape of the Fermi surface (FS) has great influence in the LDOS. In Fig. 4.12 different Fermi surfaces in two dimensions for isotropic and anisotropic systems are shown. For a complete spherical surface the corresponding Green's function for $\left(\omega=\epsilon_{F}\right)$ is a spherical wave decaying with $\left|x-x^{\prime}\right|^{\frac{1}{2}}$ in amplitude. But for anisotropic surfaces the wave functions (see Eqn. (4.22)) can interfere constructively in some beam-like regions and especially the amplitude does not decay strongly in those beams. ${ }^{7}$ Weismann concluded that

$$
\begin{aligned}
& \text { "... this implies that electrons emitted from point-like sources or scattered at point-like defects do not } \\
& \text { propagate in a spherical wave like in free space, but are in general focused in preferential directions and are } \\
& \text { detectable in much larger distances from the source than in the case of an isotropic band structure." } \\
& \text { (PhD thesis: "Scanning Tunnelling Spectroscopy of Subsurface Magnetic Atoms in Copper, Electron } \\
& \text { Focusing and Kondo Effect", p. 25, Weismann, 2008, [Wei08]) }
\end{aligned}
$$

Furthermore one can show that also away from the Fermi energy the electron propagation is beam-like with non(weak)-decaying amplitude. As a conclusion Thomas-Fermi theory of screening cannot be applied for samples with anisotropic Fermi surfaces. The electron propagation is focused on narrow beams weakly-decaying in amplitude.

The Fermi surface of the host metal in this work, copper, is spherical with eight necklike band gaps in the L-directions (See Fig. 4.13). These small deviations already lead to electron focusing (See Fig. 4.14).

For the quantitative calculation of the free Propagator $G_{x, x^{\prime}}^{0}(\omega)$ for this work the linear combination of atomic orbitals technique (LCAO) was used. In particular, the surface

\footnotetext{
${ }^{6}$ Assuming that the main characteristics of the Green's function can be derived by concentrating on the imaginary part of the Green's function

${ }^{7}$ Using $u(k)=1$.
} 


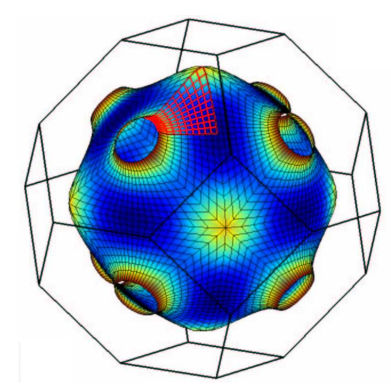

Figure 4.13: The Fermi surface of copper calculated with LCAO showing eight neck-like band gaps in the L-directions. Figure taken from the $\mathrm{PhD}$. thesis of $\mathrm{A}$. Weismann [Wei08] .

of the crystal and a realistic decay into the vacuum were taken into account. Again the starting point is the spectral representation from the band structure $\epsilon_{\mathbf{k}, \nu}$ of copper and the wave functions $\Psi_{\mathbf{k}, \nu}$ :

$$
G_{\mathbf{x}, \mathbf{x}^{\prime}}^{0}(\omega)=\sum_{\nu} \int_{1 . B Z} d^{3} \mathbf{k} \frac{\Psi_{\mathbf{k}, \nu}^{*}\left(x^{\prime}\right) \Psi_{\mathbf{k}, \nu}(x)}{\omega-\epsilon_{\mathbf{k}, \nu}+i \eta} .
$$

The band structure $\epsilon_{\mathbf{k}, \nu}$ of the hybridized s-d conduction band of copper was calculated by the LCAO approach with parameters taken from Ref. [Pap86]. The wave functions $\Psi_{\mathbf{k}, \nu}$ were derived from plane waves while reflections of the electrons by the surface and a realistic decay into the vacuum were taken into account, see Appendix B.1 for the explicit calculations. One important observation is that the presence of the surface leads to oscillations in the free LDOS at the impurity position (see Fig. 4.11c), which can be understood as a standing wave pattern of electrons being reflected at the surface. These oscillations have a periodicity proportional to $k_{F}$.

$$
\begin{array}{l|l}
\text { Interactions and Kondo physics } & 4.4 .2
\end{array}
$$

To include the strong interactions that give raise to the Kondo effect, the impurity is modelled with the SIAM. The electron bath at the impurity position is given by the band structure of copper (given by the LCAO calculations). The Green's function at the surface depending on the impurity position can then be calculated with the Dyson equation.

\section{Equation of motion technique for the SIAM}

In order to combine the free electron propagation from the surface to the impurity in the copper crystal and the scattering at the impurity the equation of motion technique is used, see Eqn. (2.13):

$$
\omega \ll A, B \gg_{\omega}=\left\langle[A, B]_{s}\right\rangle+\ll\left[A, H_{\text {Siam }}\right]_{s}, B \gg_{\omega}
$$

Evaluating this equation for all the combinations of the operators $A, B=c_{k}, c_{k}^{\dagger}, c_{d}, c_{d}^{\dagger}$ leads to a system of coupled equations of the Green's functions, which can finally be simplified to a single equation:

$$
G_{k \sigma k^{\prime} \sigma^{\prime}}(\omega)=\frac{\delta_{k k^{\prime}}}{\omega-\epsilon_{k}}+\frac{V_{k}^{*}}{\omega-\epsilon_{k}} G_{d \sigma d \sigma}(\omega) \frac{V_{k^{\prime}}}{\omega-\epsilon_{k}^{\prime}}
$$



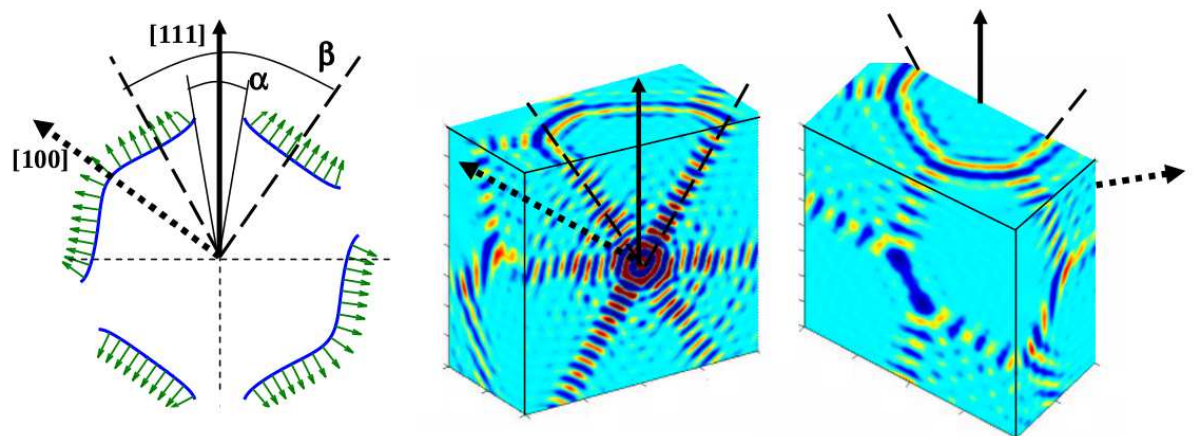

Figure 4.14: a) Fermi surface (cross section) of copper showing areas of reduced curvature b,c) corresponding propagator of copper: strong electron focusing. Figure taken from the PhD. thesis of A. Weismann [Wei08].

For the explicit calculations please see Refs. [Hew93, Dar09]. Inserting the Green's function $G_{k \sigma k^{\prime} \sigma^{\prime}}^{0}$ of the conduction electrons given by Eqn. (B.5) and introducing the T-matrix leads to:

$$
G_{k \sigma k^{\prime} \sigma^{\prime}}(\omega)=G_{k \sigma k^{\prime} \sigma^{\prime}}^{0}(\omega)+G_{k \sigma k^{\prime} \sigma^{\prime}}^{0}(\omega) T_{k k^{\prime}}(\omega) G_{k \sigma k^{\prime} \sigma^{\prime}}^{0}(\omega)
$$

In this case the T-matrix is defined as:

$$
T_{k k^{\prime}}(\omega)=\left\langle k|T(\omega)| k^{\prime}\right\rangle=V_{k}^{*} G_{d d}(\omega) V_{k^{\prime}}
$$

$G_{d d}(\omega)$ is the impurity Green's function which calculation is described in the following Sec. 4.4.2. Depending on the definition Eqn. (4.26) is usually called Dyson equation. However in this form the self-energy in the T-matrix is still reducible and therefore one has to be careful with this terminology. See Ref. [Dar09] for further discussion on this issue. Transforming Eqn. (4.26) to real space yields:

$$
G_{\mathbf{x}, \mathbf{x}^{\prime}}(\omega)=G_{\mathbf{x}, \mathbf{x}^{\prime}}^{0}(\omega)+\iint d \mathbf{z}^{\prime} d \mathbf{z} G_{\mathbf{x}, \mathbf{z}^{\prime}}^{0}(\omega) T_{\mathbf{z}, \mathbf{z}^{\prime}}(\omega) G_{\mathbf{z}^{\prime}, \mathbf{x}^{\prime}}^{0}(\omega)
$$

with

$$
T_{\mathbf{z}, \mathbf{z}^{\prime}}(\omega)=\sum_{k k^{\prime}} \psi_{k}(\mathbf{z}) \psi_{k^{\prime}}^{*}\left(\mathbf{z}^{\prime}\right) T_{k k^{\prime}}(\omega)
$$

In the following a true point-scatterer at position $\mathbf{x}_{i m p}$ is assumed, leading to

$$
G_{\mathbf{x}, \mathbf{x}^{\prime}}(\omega)=G_{\mathbf{x}, \mathbf{x}^{\prime}}^{0}(\omega)+G_{\mathbf{x}, \mathbf{x}_{i m p}}^{0}(\omega) V^{2} G_{d d}\left(\omega, \mathbf{x}_{i m p}\right) G_{\mathbf{x}_{i m p}, \mathbf{x}^{\prime}}^{0}(\omega) .
$$

In the last equation $\mathbf{x}_{i m p}$ is inserted into the arguments of $G_{d d}\left(\omega, \mathbf{x}_{i m p}\right)$ to underline that the system is not truly decoupled as $G_{d d}$ still depends on the free Green's function $G_{\mathbf{x}_{i m p}, \mathbf{x}_{i m p}}^{0}(\omega)$ at the impurity position. 


\section{Evaluating the impurity Green's function with the NRG}

For the evaluation of the Green's function of the impurity $G_{d d}\left(\omega, \mathbf{x}_{i m p}\right)$ the NRG as described in Sec. 3.2.3 is applied. The bath is expressed via the hybridization function that is given by the free Greens function of the copper crystal:

$$
\Delta(\omega)=\pi \sum_{k} V_{k}^{2} \delta\left(\omega-\epsilon_{k}\right)=-V^{2} \operatorname{Im} G_{\mathbf{x}_{i m p}, \mathbf{x}_{i m p}}^{0}(\omega)
$$

The free Green's function at the impurity position is given by the LCAO calculations via the band structure $\epsilon_{k}$. For the evaluation of the Green's function the "self-energy trick" that maintains the Friedel sum rule for the peak height of the Kondo resonance (See Sec. 3.2.3) is used. The SIAM has three free parameters $\epsilon_{d}, U$ and $V$. From Ref. [UKSZ00] the impurity level is set to $\epsilon_{d}=-0.8 \mathrm{eV}$ and $\Delta\left(\epsilon_{F}\right)=0.2 \mathrm{eV}$ is taken from Ref. [LCNJ06], so that hybridization is extracted accordingly to be $V=0.447$. The interaction $U$ is adjusted by comparison to the experiment (see Sec. 4.5).

The oscillatory behaviour of the unperturbed Green's functions $G^{0}\left(\mathbf{x}, \mathbf{x}^{\prime}, \omega\right)$ at the Fermi energy transfers to a resonance height and width oscillating with the depth of the impurity (see Fig. 4.11d). The oscillations in the peak height are in accordance with the Friedel sum rule $G_{d d}\left(\epsilon_{F}, \mathbf{x}_{i m p}\right)=\frac{1}{\pi \Delta\left(\epsilon_{F}, \mathbf{x}_{i m p}\right)}$.

\section{Phenomenological expression $\mid 4.4 .3$}

For a comparison with the experiment a phenomenological expression for the LDOS is helpful, especially for the extraction of key quantities, e.g. the Kondo temperature. In a first computation Újsághy et al. [UKSZ00] derived Fano line shapes [Fan61] for the LDOS. In their work the $G_{d d}\left(\omega, \mathbf{x}_{i m p}\right)$ was approximated by a Lorentzian $G_{d d}\left(\omega, \mathbf{x}_{i m p}\right)=$ $\frac{\Gamma_{F}}{\left(\omega-\varepsilon_{K}\right)^{2}+\Gamma_{F}^{2}}$. Here $\varepsilon_{K}$ is the position of the Kondo resonance and $\Gamma_{F}$ is proportional to the resonance width, see Sec. 4.2.2. With some approximation one can then derive Fano functions for the LDOS, see Appendix B.2 and Ref. [Dar09]. Fano line shapes have been originally derived for the spectra of a single level (state) coupled to a continuum of states. They consist of four major line shapes (see lower panel of Fig. 4.15), that are usually parametrized by a single value $q$.

However, the additional approximations by Ujjághy are not necessary as one can directly insert $G_{d d}\left(\omega, \mathbf{x}_{i m p}\right)$ into the Dyson equation in real space, see Eqn. (4.30). The free propagation of band electrons can be modelled by an energy-independent phase factor $G^{0}(r) \approx e^{i \phi_{q}(r) / 2}$ with $r=\left|\mathbf{x}-\mathbf{x}_{\mathrm{imp}}\right|$. The energy independence is justified as the energy is nearly constant around the Fermi energy, see Fig. 4.11c. The frequency of the oscillation in the free Green's function is given by the parameter $\phi_{q}(r)=2 k_{\mathrm{F}} \cdot r$, which determines the line shape. Using only these two approximations (energy-independent free 
Green's functions and Lorentzian T-matrix) the following phenomenological formula for the Lorentz/Fano line shapes is derived: ${ }^{8}$

$$
\begin{aligned}
\Delta \operatorname{LDOS}_{\text {Lorentz/Fano }}(\omega, r) & =\operatorname{LDOS}_{\text {Lorentz/Fano }}^{\mathrm{int}}(\omega, r)-\operatorname{LDOS}_{\text {Lorentz/Fano }}^{\mathrm{free}}(\omega, r) \\
& \propto \operatorname{Im} G_{\mathbf{x}, \mathbf{x}_{i m p}}^{0}(\omega) V^{2} G_{d d}\left(\omega, \mathbf{x}_{i m p}\right) G_{\mathbf{x}_{i m p}, \mathbf{x}}^{0}(\omega) \\
& \propto \operatorname{Im}\left[i e^{i \phi_{q}(r)} \frac{i \Gamma_{F}}{\omega-\varepsilon_{K}+i \Gamma_{F}}\right]
\end{aligned}
$$

A better phenomenological formula can be derived if one models the impurity Green's function by the Frota form (Eqn. (4.15)), that was shown to give a better agreement with the Kondo resonance (see Sec. 4.2.2 ). With the Frota form in the impurity Green's function $G_{d d}\left(\omega, \mathbf{x}_{i m p}\right)$ the LDOS change in distance $r$ reads:

$$
\Delta \operatorname{LDOS}_{F r o t a}(\omega, r) \propto \operatorname{Im}\left[i e^{i \phi_{q}(r)} \sqrt{\frac{i \Gamma_{F}}{\omega-\varepsilon_{K}+i \Gamma_{F}}}\right] .
$$

In Fig. 4.15 the influence of the different modelling of the impurities are shown. In the left upper panel the $\Delta$ LDOS of a frequency independent scatterer is shown $-G_{d d}(\omega)=$ const. The scatterer introduces well-known Friedel oscillations in the $\Delta$ LDOS proportional to $k_{F}$.

$$
\Delta \operatorname{LDOS}_{\text {const }}(\omega, r) \propto \operatorname{Im}\left[i e^{i \phi_{q}(r)}\right]=\sin \left(\phi_{q}(r)\right) .
$$

In the central and left upper panel the scattering at an impurity modelled with a Lorentzian (centre) and Frota form (right) are shown. Again Friedel oscillations occur as already seen for the energy-independent scattering, but the signatures in the energy are much richer. The lower panel show single $\Delta$ LDOS spectra at different positions A,B,C and D. The four single spectra show all four major line shapes. For the Lorentzian modelled impurity these are very similar to Fano line shapes discussed above. ${ }^{9}$ Nevertheless, the direct analysis of the LDOS does not show a big qualitative difference between the Frota and Lorentzian modelling. A closer look at the phase gives a better way to distinguish between these two forms. In Fig. 4.16 the phase of the Green's function $\phi=\arg \left(G_{x x}(\omega)\right)$ is shown for the two different forms at position A. One can clearly see that the phase shift for the Lorentzian is $\pi$, where the true Kondo resonance modelled by the Frota form will lead to a phase shift of $\approx \pi / 2$. So analysing the phase shift gives a possible approach to distinguish between Kondo scattering and other (single particle) scattering.

This combination of Frota form and energy-independent free Green's function turned out to give a very good approximation for the NRG/LCAO Green's functions. However, for the NRG/LCAO approach the only free parameter is the interaction $U$ which determines the Kondo temperature and resonance width. For the phenomenological formula the resonance width $\Gamma_{F}$ and the Fermi wavevector $k_{f}$ have to be fitted. Furthermore the

\footnotetext{
${ }^{8}$ The $\triangle \operatorname{LDOS}(\omega, r)$ denotes the difference of the LDOS between the free system without the impurity and the interaction system with an impurity. In the experiment the signal of the free system is also always subtracted and therefore this quantity gives directly the measured data.

${ }^{9}$ In order to derive the real Fano line shapes a few more approximation have to be done. See Appendix B.2 for more details.
} 


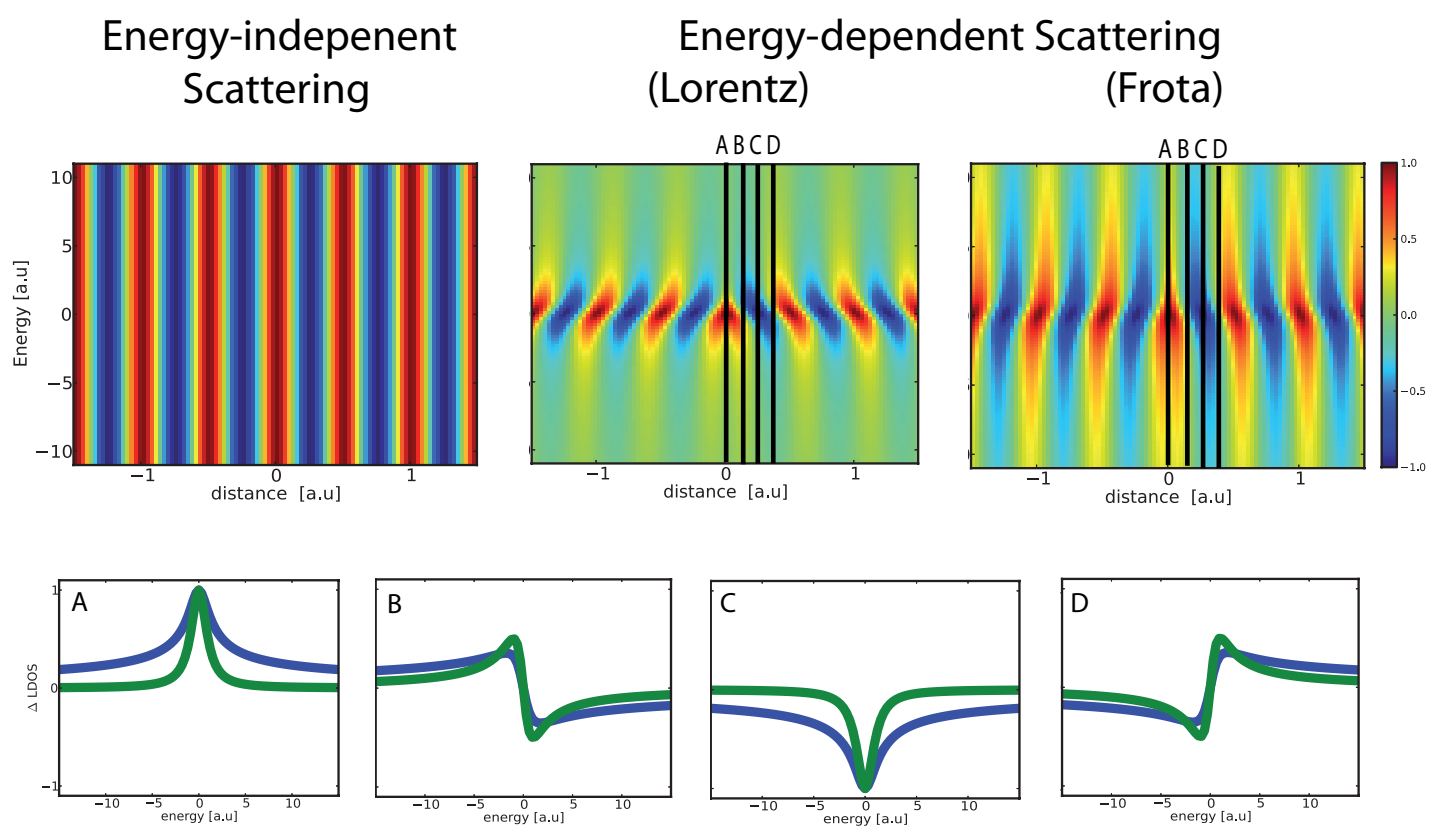

Figure 4.15: Schematic pictures for the influence of the Kondo resonance to the LDOS. In the upper panel the $\Delta$ LDOS is shown in colour code in dependence of the distance to the impurity and the energy. The LDOS is shown for an energy-independent scatterer (left, Eqn. (4.35)), an energy-dependent scatterer modelled with a Lorentzian (centre, Eqn. (4.33)) and an Kondo impurity modelled with a Frota form (right, Eqn. (4.34)). In the lower panel single LDOS spectra at specific positions A,B,C and D are shown. The green curves belong to the Lorentzian model and the blue curves to the Frota model. These line forms are very similar to Fano line shapes.

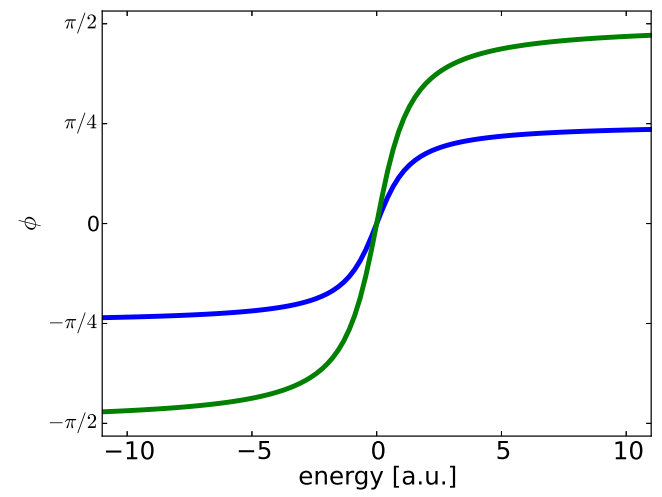

Figure 4.16: A comparison of the phase shift between the Lorentzian (green) and Frota (blue) form. The Lorentzian shows a phase shift of $\pi$ (from $-\pi / 2$ to $\pi / 2$ ). The Frota form shows only a phase shift of $\pi / 2$ (from $-\pi / 4$ to $\pi / 4)$.

amplitude oscillations due to the Friedel oscillations at the surface are not included in the phenomenological formula and have to be fitted individually for every single distance to the surface. For the fit to the experimental data an additional linear voltage slope $\beta \epsilon+\gamma$ is added to account for additional, approximately energy independent background scattering processes. 


\section{Comparison of simulation and experiment 4.5}

At first the NRG/LCAO approach is used to make a comparison with the experiment as this approach has only one free parameter. In the second part the derived phenomenological formula that allows a better fitting is used to extract the Kondo temperatures from the experiment and to analyse the phase shift.

\section{Local density of states at the surface $\mid 4.5 .1$}

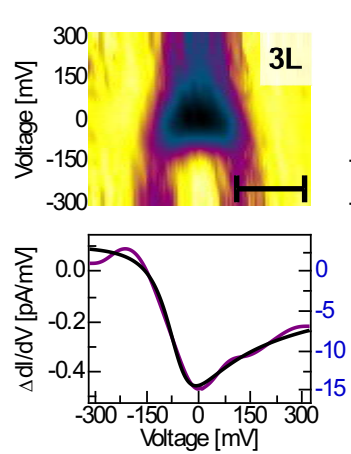

3L
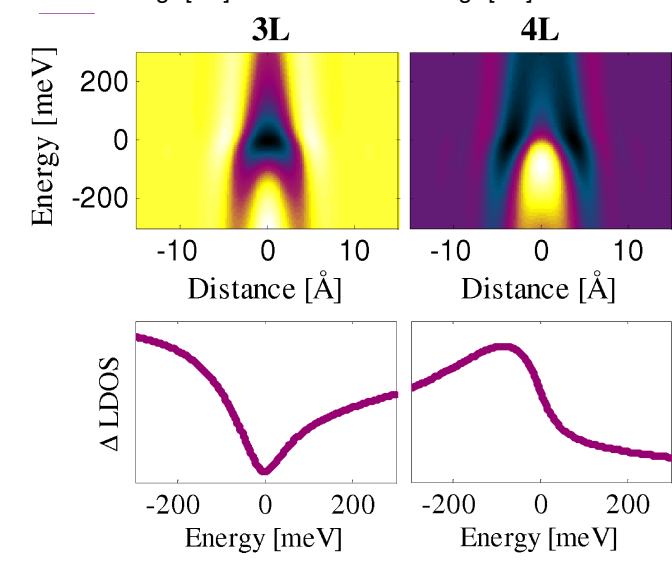

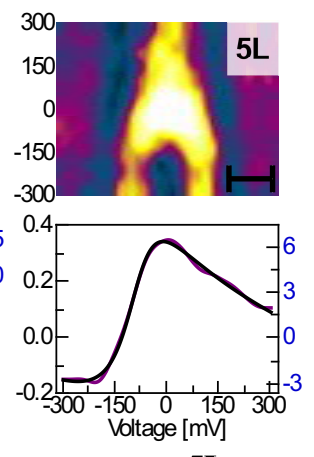

$5 \mathrm{~L}$
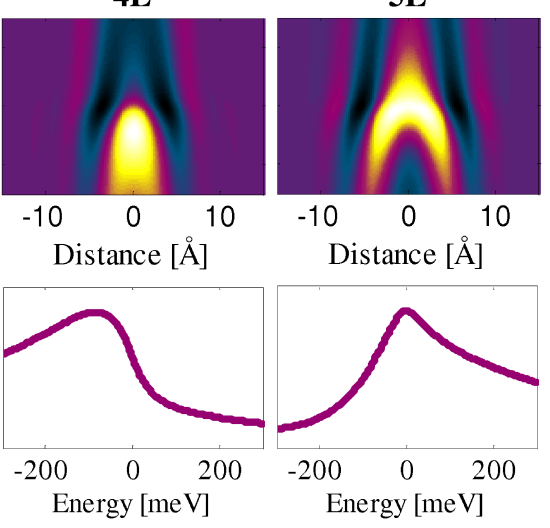

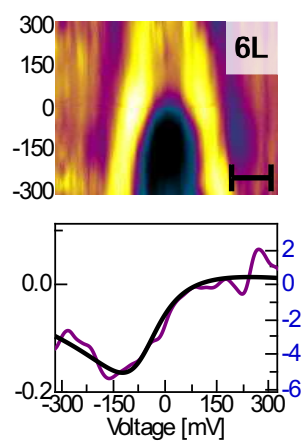

6L
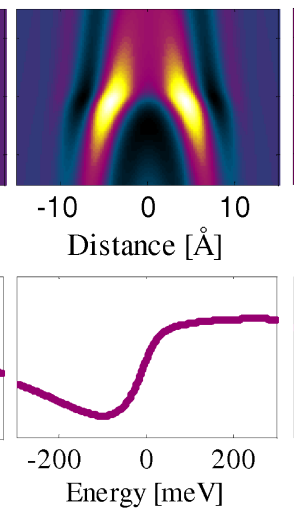

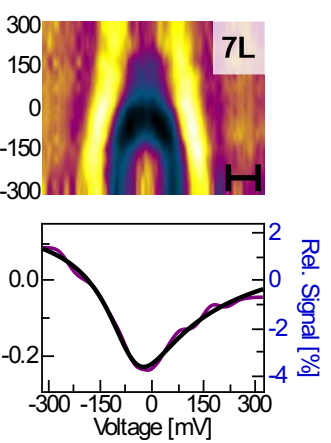

$7 \mathrm{~L}$
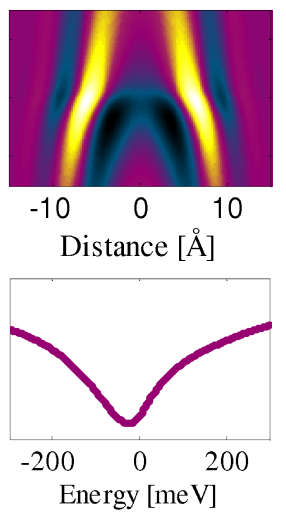

Figure 4.17: (Published in $\left[\mathrm{PWD}^{+} 11\right]$.) (Upper panel) Spectrum sections $\Delta d I / d V=$ $d I / d V-d I_{0} / d V$ along the [010] direction are shown in the upper part for monolayers $d=3-7 L$. A strong change in the scattering behaviour can be seen for all impurity depths. The black bar corresponds to a length of $0.5 \mathrm{~nm}$ for every section. Single $\Delta d I / d V$ spectra (purple curve) measured with a tip position direct above the impurity $y=0$ are depicted in the lower part. To illustrate the relative amplitude of the Kondo signal, the data are normalized to the differential conductance of the free surface (blue scale on the right side). The black curves are obtained by fitting the phenomenological expression of the Kondo resonance to the differential conductance (see Sec. 4.4.3). Note that the colour scale for the spectroscopic data is similar to that used in Fig. 4.10, except the range varies, and is set by the corresponding single spectra.

(Lower panel) The simulation results of the LDOS for the same impurity depths as in the experiment calculated with the combination of LCAO/NRG. Only the interaction $U$ on the impurity level has been adjusted. One can clearly see the same oscillatory pattern as in the experiment. Also the single spectra show the same line shapes, that are oscillatory with depth. (pe) 
The upper panel of Fig. 4.17 shows STS-data of buried Co atoms for different impurity depth $(d=3-7 M L)$. The lateral variations of $d I / d V$ are again depicted as sections (upper part of the panel). In all spectra in the different monolayers a signature of energydependent scattering is visible. All show strong effects at the Fermi energy $(\omega=0)-$ at the position of the Kondo resonance. One can clearly observe the periodicity, compare the $3 M L$ and the $7 M L$, as predicted already by Ujsághy et al. [UKSZ00]. This can especially be seen by comparing single spectra (purple curves) measured directly above the impurities $(y=0)$ (lower part of the upper panel).

In the lower panel of Fig. 4.17 the simulation data with the combined NRG/LCAO approach are shown. As the values for energy and hybridization of the localized orbital are taken from ab-initio results (See Sec. 4.4.2), the only free parameter left, the Coulomb interaction $U$, is adjusted by comparison to the experiment. The best agreement for cobalt is obtained by a value of $U=1.2 \mathrm{eV}$ (and for iron for $U=2.4 \mathrm{eV}$ ). One should point out that for cobalt one is not in the true Kondo regime that is given by $\frac{U}{\pi \Delta\left(\epsilon_{F}\right)} \gtrsim 3$ (See Sec. 4.2.2). The calculated LDOS sections are in excellent agreement with the measured data. The experimentally observed periodicity (compare a 3rd and 7th layer impurity) is also found in the simulation. In particular, the comparison of single spectra, e.g. at $y=0$. shows very good agreement. The (approximate) Fano line shapes can be clearly seen. One should emphasize again that only the impurity interaction $U$ has been adjusted to fit the experiment.

Furthermore the comparison between Co and Fe data demonstrates that both impurity species show similar behaviour on completely different energy scales (for instance compare the 5th layer Fe in Fig. 4.10 with a 5th layer Co in Fig. 4.17). This is one hint that the universal approach with the SIAM neglecting specific characteristics of the individual impurities (See Sec. 4.1) is justified.

\section{Kondo temperatures 4.5 .2}

The rich spatial and spectroscopic information of the measured interference patterns allow further investigation of the Kondo resonance. The resonance width is proportional to the Kondo temperature, see Sec. 4.2.2. Due to the very good agreement of the NRG/LCAO calculations with the derived phenomenological form, see Eqn. (4.34), a fit of the experimental data with the phenomenological form for all lateral positions and a constant impurity depth is performed. Averaging over all different depths a Kondo temperature $T_{K}$ for Fe impurities of $32(16) \mathrm{K}$ and for Co of $655(155) \mathrm{K}$ is obtained. Results from macroscopic bulk measurements [DS68] give similar values, see Fig. 4.18. Investigating the Kondo temperature as a function of the lateral position for a constant impurity depth an unexpected variation of the resonance width is found. As an example the 5th layer Fe impurity (Fig. 4.10c) shows a variation from $T_{K}(\mathrm{~A})=29(9) \mathrm{K}, T_{K}(\mathrm{~B})=35(24) \mathrm{K}$, $T_{K}(\mathrm{C})=42(18) \mathrm{K}$ and $T_{K}(\mathrm{D})=44(26) \mathrm{K}$ from position $\mathrm{A}$ to $\mathrm{D}$. This behaviour is not included in the theory so far. One possible explanation employs the actual orbital structure of the d-level in $\mathrm{Fe}$ or $\mathrm{Co}$, which can lead to a dependence of the T-Matrix on the propagation direction, see Sec. 4.6.1 for further discussion. 


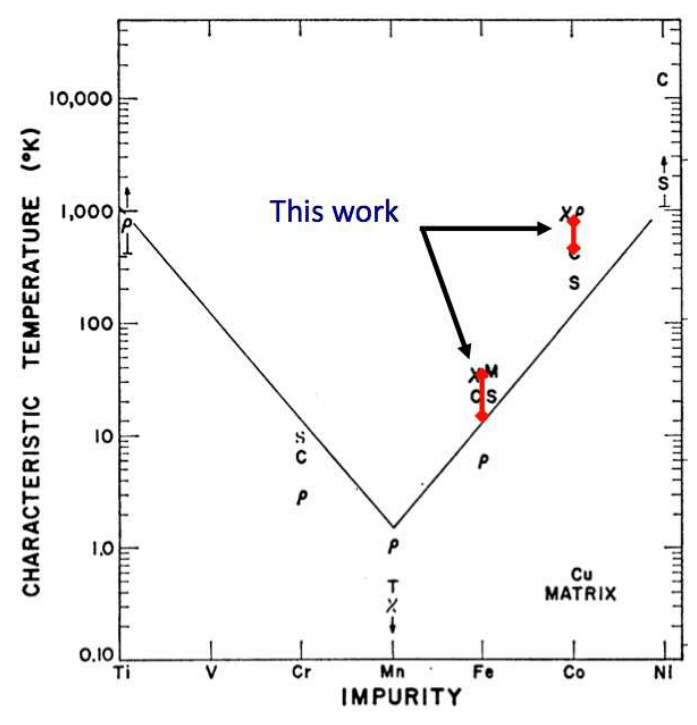

Figure 4.18: (Adapted from Fig. 2 in Ref. [DS68]) The extracted Kondo temperatures from the experiments: Fe impurities of $32(16) \mathrm{K}$ and for Co of $655(155) \mathrm{K}$ in comparison to Kondo temperatures from macroscopic bulk measurements. The $\mathrm{x}$-axis represents the different impurity elements and the y-axis the related bulk Kondo temperatures.

\section{Phase shift 4.5 .3}

Going further, not only the long range signature of the scattering amplitude are investigated but also its phase. During the analysis it turned out that the widely used (complex) Lorentzian approximation of the Kondo resonance does not give the best description to both the experimental and theoretical data, see Sec. 4.2.2. As stated beforehand the shift of the scattering phase due to the Kondo resonance is strongly overestimated as a Lorentzian always results in a phase shift of $\pi$. The Frota form and the NRG calculations give a phase shift of only $\pi / 2$. As one can see in Fig. $4.10 \mathrm{~b}$ the phase shift measured in experiment is also smaller than $\pi$.

\section{Analysis of the data 4.6}

Although the very good agreement of experiment and simulation seems to be very convincing one should discuss the chosen model and the simulation results more carefully. At first the effective model is compared to more elaborate models for Fe and Co impurities. Then it is argued that the measured signatures are really long range Kondo signature. In the end it is discussed if the Kondo cloud has been measured.

\section{Effective model $\mid 4.6 .1$} "A theory has only the alternative of being right or wrong. A model has a third possibility: it may be right,
but irrelevant."

(Article: "The Origin of Biological Information", Eigen, 1973,[Eig73])

Obviously the effective impurity modelling of Co and Fe as a single level is a severe approximation and can only be justified due to the universality of the Kondo effect. In a 
first step to a more realistic model all five $3 d$-orbitals have to be taken into account. A model Hamiltonian is then given by a multi-orbital Anderson model [BCP08]

$$
\begin{aligned}
\mathcal{H}_{\mathrm{AIM}}= & \sum_{k \alpha} \epsilon_{k} c_{k \alpha}^{\dagger} c_{k}+\sum_{k \alpha \alpha^{\prime}}\left(V_{k \alpha^{\prime} \alpha} d_{\alpha}^{\dagger} c_{k \alpha^{\prime}}+V_{k \alpha^{\prime} \alpha}^{*} c_{k \alpha^{\prime}}^{\dagger} d_{\alpha}\right) \\
& +\sum_{\alpha} \epsilon_{\alpha} d_{\alpha}^{\dagger} d_{\alpha}+\sum_{\alpha_{1} \alpha_{2} \alpha_{3} \alpha_{4}} U_{\alpha_{1} \alpha_{2} \alpha_{3} \alpha_{4}} d_{\alpha_{1}}^{\dagger} d_{\alpha_{2}}^{\dagger} d_{\alpha_{3}} d_{\alpha_{4}}
\end{aligned}
$$

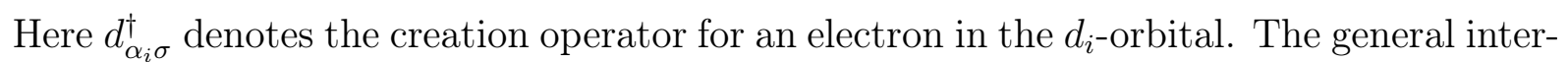
action term is usually split into an intraorbital term $(U)$, an interorbital Coulomb repulsion $\left(U^{\prime}\right)$ and an exchange term $(J)$, that is usually further split into an Heisenberg exchange term (Hund's coupling) and an orbital exchange term. As mentioned in Sec. 4.2.1, the embedding of the impurity into the crystal leads to crystal field splitting of the orbital degeneracy. For the case of $\mathrm{Co}$ and $\mathrm{Fe}$ in $\mathrm{Cu}$ one usually gets threefold-degenerate $t_{3 g}$ orbitals and twofold-degenerate $e_{g}$ orbitals. Unfortunately a full NRG calculation of the full five-orbital problem is not possible due to large local basis in the conduction channels.

In a simpler generic two orbital model it could be numerically shown that the Hund's coupling alone ${ }^{10}$ leads to a dramatic decrease of the Kondo temperature $T_{K}$ and a resulting narrowing of the Kondo resonance [PB05, NCH10]. This was later explained in a simple picture by adopting Anderson's poor man scaling [NC09]. Nevertheless it does not change qualitatively the Kondo physics, just the energy scale (Kondo Temperature) is somehow rescaled. Therefore for the interpretation of the extracted Kondo temperatures the neglected Hund's coupling should be remembered.

In a very recent study $\left[\mathrm{STW}^{+} 12\right]$ an ab-initio calculation of the full problem of Co in $\mathrm{Cu}$ with a newly developed Quantum Monte Carlo algorithm in combination with DFT was performed. The DFT calculation (not including any surface) gave an averaged screened Coulomb interaction of $U=4 \mathrm{eV}$ and $J=0.9 \mathrm{eV}$. The average occupation of the orbitals is between $n=7$ and $n=8$ and an impurity spin of $S_{z}=1(\mathrm{GGA}+\mathrm{U})$ and $S_{z}=\frac{1}{2}$ (GGA). This is in agreement with the "low spin complex" assumption of the SIAM as discussed in Sec. 4.2.1. The QMC calculations at $T=0.025$ showed in both $\left(t_{2 g}\right.$ and $e_{g}$ ) orbitally resolved DOS a very similar resonance at the Fermi energy despite the crystal field splitting. These findings support the effective modelling of the system with the SIAM, but they point out that due to the missing charge fluctuations an effective Kondo model is not appropriate. As shown in Sec. 4.5 the extracted Kondo temperatures $\left(T_{k} \approx 655 \pm 155 \mathrm{~K}\right)$ for Co are in agreement with bulk measurements. Nevertheless they do deviate from measurements of Kondo temperatures from ad-atoms. They have found to be $T_{k} \approx 54 \pm 5 K\left[\mathrm{KSD}^{+} 02, \mathrm{NKB}^{+} 08, \mathrm{MLE} 00\right]$. This large ratio of $T_{k}^{\text {in }} / T_{k}^{o n} \approx 12.1$ has also been found in the simulation of Surer et al. : $T_{k}^{i n} / T_{k}^{o n} \approx 7.4\left[\mathrm{STW}^{+} 12\right]$.

The specific situation for $\mathrm{Fe}$ in $\mathrm{Cu}$ is even more complicated. Costi et al. [CBW $\left.{ }^{+} 09\right]$ could show that for $\mathrm{Fe}$ in $\mathrm{Ag}$ and $\mathrm{Au}$ the best description is given by an effective multichannel Kondo model in the low temperature regime:

\footnotetext{
${ }^{10}$ These calculations were done with a ferromagnetic $J>0$ and $U^{\prime}=U-2 J$, which implies rotational invariance.
} 
"Remarkably, for both $\mathrm{Ag}$ and Au samples, the use of a fully screened $s=\frac{3}{2}$ three-channel Kondo model allows a quantitatively consistent description of both the resistivity and decoherence rate with a single $T_{K}$ (for each material)."

(Article: "Kondo Decoherence: Finding the Right Spin Model for Iron Impurities in Gold and Silver", Costi et al. , 2009, $\left.\left[\mathrm{CBW}^{+} 09\right]\right)$

Nevertheless the agreement for $T<T_{k}$ is also for a spin- $\frac{1}{2}$ one channel Kondo model very good for the quantities studied.

Spin-orbit interactions are strongest in transition metal compounds with $5 \mathrm{~d}$ (and weaker in $4 d$ ) electrons. In $3 d$ compounds as investigated in this work the Coulomb interactions are dominant and spin-orbit interactions are believed to be negligible, see Ref. [MAVB11, Col12] for further discussion.

\section{Observing a real Kondo signature ? $\quad 4.6 .2$}

The Kondo effect in the LDOS is characterized by the Kondo resonance at the Fermi energy that forms below the Kondo temperature $T_{K}$. In all the analysed spectra a strong signal has been found at the Fermi energy. Unfortunately the performed experiments do not allow to record the LDOS with varying temperature. With increasing temperature it is more and more difficult to keep the focus of the STM above the single impurity. ${ }^{11}$ However, in a different control experiments with buried non-magnetic Ag impurities no signatures at the Fermi energy could be observed [PWWU12].

Besides the strong signal at the Fermi energy there are further hints for the observation of a long-range Kondo signature. Using the recently observed focussing effect, the simulation of the spectra at the surface shows the same oscillatory behaviour as in the experiment. As shown in Sec. 4.4.3 for the characteristic (Fano) line shapes an energydependent scatterer is needed. However, just from these line shapes the specific form of the energy-dependent scatterer (Lorentz or Fano) is hard to distinguish.

In Sec. 4.5.3 the phase shift was further analysed. It was shown that Kondo scattering is accompanied by a phase shift smaller than $\pi / 2$ which was also found in the experiment. This allows to distinguish between different energy-dependent scatterers and further supports the finding of a long-range Kondo signature.

Finally, the extracted 'effective' Kondo temperatures for Fe and Co are consistent with bulk measurements. However, as denoted beforehand, these values have to be interpreted carefully. Nevertheless the relation between bulk and surface Kondo temperatures is also consistent with a recent numeric study, see Sec. 4.6.1.

Therefore it can be stated that the experimental data is consistent with the observation of a long range Kondo signature.

\footnotetext{
${ }^{11}$ Please see Refs. [Wei08, Pru08, PWWU12] for additional information.
} 


\title{
Measuring the Kondo cloud?
}

\begin{abstract}
"One ongoing debate concerns the so-called Kondo cloud. The many electrons that are involved in the spinflip processes [...] combine to build the Kondo resonance. The Kondo cloud consists of electrons that have previously interacted with the same magnetic impurity. Since each of these electrons contains information about the same impurity, they effectively have information about each other. In other words, the electrons are mutually correlated. The holy grail for research on the Kondo effect is to know whether it is possible to measure and control the Kondo cloud."
\end{abstract}

(Review article: "The revival of the Kondo effect", Kouwenhoven and Glazman, 2001, [KG01])

After having shown that the data is consistent with a long range signatures of the Kondo effect, a natural questions is to ask "Is this a measurement of the Kondo cloud?".

Unfortunately the measured quantity in this work, the spectral function, does not give a direct access to the Kondo cloud. Using the Dyson Equation, see Eqn. (4.28), the full Green's function can be expressed by the free Green's function:

$$
G(\mathbf{r}, \mathbf{r}, \omega)=G^{0}(\mathbf{r}, \mathbf{r}, \omega)+\mathbf{G}^{\mathbf{0}}\left(\mathbf{r}, \mathbf{r}^{\prime}, \omega\right) \mathbf{T}\left(\omega, \mathbf{r}^{\prime}, \mathbf{r}\right) \mathbf{G}^{\mathbf{0}}\left(\mathbf{r}^{\prime}, \mathbf{r}, \omega\right)
$$

For the SIAM the T-Matrix has a very weak spatial dependency. This is due to the universality of the Kondo effect. It was shown that the impurity position has a very small oscillatory effect to the Kondo resonance mainly due to surface effects. For a constant DOS at all impurity positions there is no space dependence at all. Therefore the main spatial dependencies of $G(\mathbf{r}, \mathbf{r}, \omega)$ are due to the free Green's function $G^{0}\left(\mathbf{r}, \mathbf{r}^{\prime}, \omega\right)$.

"Note that $G(r, r, \omega)$ itself has trivial r-dependence, determined entirely by $G^{0}(r)$. Only the frequency-
dependence reflects the Kondo physics. However, once we integrate over $\omega$, this $\omega$-dependence introduces
some interesting r-dependence."
(Review article: "The Kondo screening cloud: what it is and how to observe it", Affleck, 2009, [Affo9])

Integrating the spectral function over all frequencies up to the Fermi energy yields the charge density:

$$
\rho(r)=-\frac{2}{\pi} \int_{-\infty}^{\epsilon_{F}} \rho(\omega, r) d \omega
$$

Affleck et al. [ABS08] could further show that for a Kondo model with a spherical symmetric dispersion relation the charge density oscillations are given by an universal function $F\left(r / \xi_{K}\right)$ :

$$
\rho(r)-\rho_{0}=\frac{C_{D}}{r_{D}}\left[\cos \left(2 k_{f} r-\pi D / 2+2 \delta_{P}\right) F\left(r / \xi_{K}\right)-\cos \left(2 k_{f} r-\pi D / 2\right)\right] .
$$

Here $D$ denotes the dimension of the system with $C_{D}$ given by $C_{1}=1 /(2 \pi), C_{2}=1 /\left(2 \pi^{2}\right)$ and $C_{3}=1 /\left(4 \pi^{2}\right)$ and $\delta_{P}$ is the phase shift at the Fermi surface due to potential scattering. The universal function $F\left(r / \xi_{K}\right)$ approaches the values +1 for $r \ll \xi_{K}$ and -1 for $r \gg \xi_{K}$. The experiments can provide spectral data only for very short distances so that a comparison to such a function is hopeless. For the identification of the Kondo cloud for buried impurities with the STM a much stronger focussing or much higher experimental resolution would be needed so that even signatures from deeply buried impurities can be 
analysed.

A numerical simulation of these charge densities then also has to include the focussing effect. Thus one has to take the real free Green's functions of the copper crystal and try to find a similar dependency on such a universal function like in Eqn. (4.39). This numerical integration of all frequencies up to the Fermi energy will demand also for much higher quality data of the free Green's functions of the copper crystal as even frequencies far below the Fermi energy have significant influence. Furthermore it will be very difficult to distinguish the charge oscillations from the ones that are induced by the proximity to the surface. 


\section{Dynamic spin structure factor of the one-dimensional Heisenberg model

The Heisenberg model was proposed by Heisenberg in 1928 [Hei28] to explain ferromagnetism via an effective spin model. For one-dimensional chains it was formally solved by Bethe in 1931 [Bet31], but the calculation of dynamic quantities still poses a big challenge. In higher dimensions no analytical solutions exist. Recently, renewed interest in dynamic quantities of spin chains and ladders was triggered by new developments in neutron scattering experiments that can now provide high resolution data, see for example Refs. [TRR ${ }^{+}$09, $\left.\mathrm{JOK}^{+} 11, \mathrm{RTC}^{+} 11\right]$. These systems can be modelled with the one-dimensional Heisenberg model and extended Heisenberg models for which no analytic solutions via the Bethe ansatz exist. The goal of this project is to show that the newly developed MPS Lanczos method is capable of providing high resolution dynamic correlation functions for spin chains. The standard Heisenberg model is taken as a non-trivial test case.

In Sec. 5.1 the Heisenberg model is introduced. The Bethe ansatz solution of the model is shortly presented with a focus on the 2 -spinon excitations. Furthermore the dynamic spin structure factor is defined and the known asymptotics from the Bethe ansatz are briefly explained. In Sec. 5.2 the computation of the dynamic spin structure factor with the newly developed MPS Lanczos method is presented. A special focus is on the precision and error analysis. The chapter closes with a comparison of different methods for the calculation of the dynamic spin structure factor in Sec. 5.3.

The content of this chapter is published as a regular article in Physical Review B $\left[\mathrm{DWH}^{+}{ }^{12}\right]$ and therefore it may be that some parts of the following chapter resemble the manuscript of Ref. [DWH $\left.{ }^{+} 12\right]$.

The work was a collaboration with Andreas Honecker, Anton Wöllert, Ian P. McCulloch, Ulrich Schollwöck and Thomas Pruschke. Andreas Honecker helped with his insight on the dynamics of the Heisenberg model. Together with Anton Wöllert the ideas for the reorthogonalization of the Lanczos vectors were developed. Ian P. McCulloch provided the MPS code. The manuscript was written mainly by me with support from Thomas Pruschke, Andreas Honecker and Ulrich Schollwöck. All authors discussed the results and commented on the manuscript. 


\section{\begin{tabular}{l|l} 
Anti-ferromagnetic spin- $\frac{1}{2}$ Heisenberg chain & 5.1
\end{tabular}}

In order to derive an effective Hamiltonian for ferromagnetism as well as anti-ferromagnetism Heisenberg assumed that only the spins of the electrons get oriented and that the spatial wavefunctions are not of importance.

"In ein neues Stadium ist der ferromagnetische Fragenkomplex getreten durch die Uhlenbeck-Goudsmitsche
Theorie des Spinelektrons. Insbesondere folgt aus dem bekannten Faktor $g \approx 2$ beim Einstein-de Haas-
Effekt (der ja eben bei ferromagnetischen Substanzen gemessen wurde), dass sich in einem ferromagnetischen
Kristall nur die magnetischen Eigenmomente der Elektronen, gar nicht die Atome orientieren." ${ }^{1}$
(Article: "Zur Theorie des Ferromagnetismus", Heisenberg, 1928,[Hei28])

Furthermore the spins are assumed to be localized on the crystal lattice and interact only via a constant exchange interaction $J$, that can be derived with some approximations from the Coulomb-interaction and the Pauli principle. The sign of the exchange interaction distinguishes between anti -and ferromagnetism. The Hamiltonian of the onedimensional spin- $\frac{1}{2}$-Heisenberg model is given by

$$
H=J \sum_{i} \mathbf{S}_{i} \mathbf{S}_{i+1}
$$

Here $\mathbf{S}_{i}$ denotes the usual spin-operator at site $i$. A positive sign of $J$ leads to an antiferromagnetic ground state and a negative sign to a ferromagnetic ground state. The following summary of results from the Bethe ansatz solution are taken from Refs. $\left[\mathrm{KMB}^{+} 97\right.$, KM98, KHM98].

\section{Ferromagnetic ground state and magnon excitations $\quad 5.1 .1$}

In the Bethe ansatz all eigenstates of the Heisenberg Hamiltonian of a spin chain with periodic boundary conditions and $N$ spins are generated from the ferromagnetic ground state $|F\rangle=|\uparrow \uparrow . . \uparrow \uparrow\rangle$ with energy $E_{F}=-J N / 4$ by multiple magnon excitations. A magnon excitation is parametrized by the number of flipped spins $r$ or the corresponding $S_{z}$ subspace.

\section{Single magnon excitations}

A single magnon excitation from the ferromagnetic ground state $|F\rangle=|\uparrow \uparrow . . \uparrow \uparrow\rangle$ with energy $E_{F}$ is created by a superposition of states with a single flipped spin $(r=1)$ in the ground state $|n\rangle=S_{n}^{-}|F\rangle$. These single magnons are eigenstates of the Hamiltonian and numbered by the momentum $k=2 \pi m / N, m=0, . . N-1$. The corresponding eigenstate is given by:

$$
\left|\Psi_{k}\right\rangle=\frac{1}{\sqrt{N}} \sum_{n=1}^{N} e^{i k n}|n\rangle .
$$

\footnotetext{
${ }^{1}$ (Free) English translation: "The complex of questions on ferromagnetism has entered a new stage due to the Uhlenbeck-Goudsmitsche theory of the spinelectron. It follows from the known factor $g \approx 2$ of the Einstein-de-Haas-effect (which has been measured in ferromagnetic materials), that in a ferromagnetic crystal only the magnetic moments of the electrons get oriented and not the atoms."
} 
and the corresponding energy of this single magnon state is given by:

$$
E-E_{F}=J(1-\cos (k)), \quad E_{F}=-J N / 4 .
$$

\section{Multiple magnon excitations}

Wavefunctions of multiple magnon excitations with $r$ flipped spins are given by:

$$
|\psi\rangle=\sum_{1 \leq n_{1}<\ldots<n_{r} \leq N} a\left(n_{1}, \ldots, n_{r}\right)\left|n_{1}, \ldots, n_{r}\right\rangle .
$$

Here the $n_{i}$ with $i=1 . . r$ give the position of the $r$ flipped spins and the sum runs over all possible combinations. The coefficients $a\left(n_{1}, \ldots, n_{r}\right)$ in terms of the momenta $k_{j}$ and phase angles $\theta_{i j}=-\theta_{j i}$ can be written as:

$$
a\left(n_{1}, \ldots, n_{r}\right)=\sum_{\mathcal{P} \in S_{r}} \exp \left(i \sum_{j=1}^{r} k_{\mathcal{P} j} n_{j}+\frac{i}{2} \sum_{i<j} \theta_{\mathcal{P} i \mathcal{P} j}\right) .
$$

The sum $\mathcal{P} \in S_{r}$ is over all $r$ ! permutations of the labels $\{1,2, \ldots, r\}$. The phase angles $\theta_{i j}$ are related to the momenta $k_{j}$ by:

$$
\begin{aligned}
2 \cot \frac{\theta_{i j}}{2} & =\cot \frac{k_{i}}{2}-\cot \frac{k_{j}}{2}, \quad i, j=1, \ldots, r \\
N k_{i} & =2 \pi \lambda_{i}+\sum_{j \neq i} \theta_{i j}, \quad i=1, \ldots, r, .
\end{aligned}
$$

where $\lambda_{i} \in\{0,1, \ldots, N-1\}$ are the Bethe quantum numbers. In order to find all the multi magnon excitations with $r$ flipped spins, one has to find those sets of Bethe quantum numbers $\left(\lambda_{1}, \ldots, \lambda_{r}\right)$ which give (real or complex) solutions $\left\{k_{j}\right\}$ and $\left\{\theta_{i j}\right\}$ of the Bethe ansatz equations, Eqn. (5.5). The corresponding eigenenergy and momentum of this eigenvector can then be calculated by:

$$
E-E_{F}=J \sum_{j=1}^{r}\left(1-\cos k_{j}\right), \quad k=\frac{2 \pi}{N} \sum_{i=1}^{r} \lambda_{i} .
$$

For a given set of Bethe quantum numbers the determination of the corresponding eigenenergy and momentum is possible. However to set up the eigenstate via Eqn. (5.4) and to calculate expectation values and correlation functions is due to the many sums a nontrivial problem.

\section{Anti-ferromagnetic chain and spinon excitations}

For the anti-ferromagnetic spin chain it is advantageous to transform the momenta $k_{i}$ and phase angles $\theta_{i j}$ to a new variable:

$$
\begin{aligned}
k_{i} & =\pi-\phi\left(z_{i}\right), \quad \phi(z) \equiv 2 \arctan z \\
\theta_{i j} & =\pi \operatorname{sgn}\left[\operatorname{Re}\left(z_{i}-z_{j}\right)\right]-\phi\left[\left(z_{i}-z_{j}\right) / 2\right] .
\end{aligned}
$$


The Bethe ansatz equations (Eqn. (5.5)) can then be rewritten in a single equation with new Bethe quantum numbers $I_{i}$ :

$$
N \phi\left(z_{i}\right)=2 \pi I_{i}+\sum_{j \neq i} \phi\left[\left(z_{i}-z_{j}\right) / 2\right], \quad i=1, \ldots, r
$$

The corresponding energy and momentum of a state with a set of Bethe numbers $I_{i}$ and corresponding solutions $z_{i}$ is given by:

$$
\begin{aligned}
\left(E-E_{F}\right) / J & =\sum_{i=1}^{r} \varepsilon\left(z_{i}\right), \quad \varepsilon\left(z_{i}\right)=d k_{i} / d z_{i}=-2 /\left(1+z_{i}^{2}\right) \\
k & =\sum_{i=1}^{r}\left[\pi-\phi\left(z_{i}\right)\right]=\pi r-\frac{2 \pi}{N} \sum_{i=1}^{r} I_{i}
\end{aligned}
$$

\section{Anti-ferromagnetic ground state}

The ground state of the anti-ferromagnetic Heisenberg chain is not the classical Néel state $|N\rangle=|\uparrow \downarrow \uparrow . . \uparrow \downarrow \uparrow \downarrow\rangle-$ as this is not an eigenstate of the system. The ground state of the anti-ferromagnetic Heisenberg chain however lies in the subspace with $N / 2$ flipped spins and can be parametrized by a set of new Bethe quantum numbers:

$$
\left\{I_{i}^{A F}\right\}=\left\{-\frac{N}{4}+\frac{1}{2},-\frac{N}{4}+\frac{3}{2}, \ldots, \frac{N}{4}-\frac{1}{2}\right\} .
$$

The anti-ferromagnetic ground state can now be regarded as the anti-ferromagnetic vacuum with $S=0, S_{z}=0$. It can be shown that the corresponding solution $\left\{z_{i}\right\}$ are all real and can be obtained from a recursion relation:

$$
z_{i}^{(n+1)}=\tan \left(\frac{\pi}{N} I_{i}+\frac{1}{2 N} \sum_{j \neq i} \phi\left[\left(z_{i}^{(n)}-z_{j}^{(n)}\right) / 2\right]\right) .
$$

It was shown that in the thermodynamic limit the ground state energy (per site) approaches the value

$$
\frac{E_{A}-E_{F}}{J N}=-\ln 2
$$

\section{Spinon excitations}

The excitations from the anti-ferromagnetic ground state are called spinons. The elementary excitations are 2-spinon excitations. They are classified by their quantum numbers into the 3 -fold degenerate triplet space with $S=1, S_{z}=-1,0,1$ and singlet space with $S=0, S_{z}=0$. By removing a single magnon from the AF ground state, one creates a 2 -spinon excitation in the $S=1, S_{z}=1$ subspace (triplet states). Mathematically these 2 -spinon excitations are given by all configurations that are formed by eliminating one Bethe quantum number from the quantum numbers $\left\{I_{i}^{A F}\right\}$ of the anti-ferromagnetic ground state and rearranging the remaining $\left\{I_{i}\right\}$ in all configurations over the expanded range $-\frac{1}{4} N \leq I_{i} \leq \frac{1}{4} N$. There are $\frac{1}{8} N(N+2)$ different 2-spinon excitations in the 
$S=1, S_{z}=1$ subspace possible. Their energy and momentum can be calculated by solving the Bethe ansatz equations via the recursion relation given by Eqn. (5.11) as it can be shown that all the solutions are real. The two remaining subspaces in the triplet subspace, the $S=1, S_{z}=-1$ and the $S=1, S_{z}=0$ have identical dispersion (energy-momentum) relations by symmetry.

The calculation of the 2-spinons states in the singlet sector $S=0, S_{z}=0$ is much more subtle as the $\left\{z_{i}\right\}$ include now complex solutions and the recursion relation Eqn. (5.11) cannot be used any more. However, due to selection rules they have no weight in the dynamic spin structure factor and therefore their calculation is omitted here, see Ref. [KM98] for further details. In Fig. 5.1 the energy-momentum (dispersion) relation of the 2 -spinon

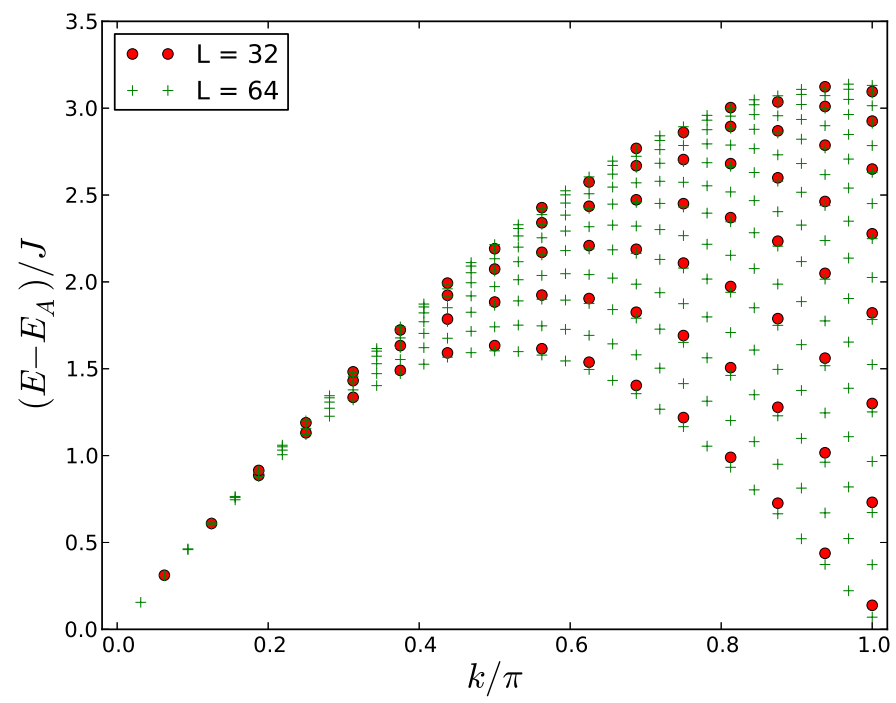

Figure 5.1: Energymomentum relation (dispersion) of the 2-spinon triplet excitations of the anti-ferromagnetic Heisenberg chain (subset $S=1, S_{z}=1$ ) calculated with the Bethe ansatz.

triplet excitations calculated by Eqn. (5.11) is shown. The energy gap of the excitations is maximal for $k=\pi / 2$ and vanishes for $k=0, \pi$. For finite systems the number of excitations is maximal for $k=\pi$. The energy bounds depending on the momentum $k$ can also be derived from the Bethe ansatz solution. They are given by:

$$
\omega_{1}=J \frac{\pi}{2}|\sin k| \quad \text { and } \quad \omega_{2}=J \pi\left|\sin \frac{k}{2}\right|
$$

The anti-ferromagnetic Heisenberg chain is gapless in the thermodynamic limit, which can be deduced from the energy bounds of the 2-spinon excitations for $k=0, \pi$. Therefore the important area law for the entanglement entropy, as described in Sec. 3.3.5, has got logarithmic corrections and the test case is known to be non-trivial for MPS. 4-spinon and higher-spinon excitations can in principle be calculated in the same spirit. However, the computations are far more complex and as the focus on this work is on the numerical calculations with MPS their computation is not further pursued in this work. 


\section{Dynamic spin structure factor $\quad 5.1 .3$}

The dynamic spin structure factor is defined by

$$
\begin{aligned}
S(k, \omega) & =-\frac{1}{\pi} \lim _{\eta \rightarrow 0} \operatorname{Im}\left\langle E_{0}\left|\mathbf{S}_{k}^{\dagger} \frac{1}{\omega-H+\mathrm{i} \eta+\mathrm{E}_{0}} \mathbf{S}_{k}\right| E_{0}\right\rangle \\
& =\frac{1}{\pi} \sum_{n}\left|\left\langle E_{n}\left|\mathbf{S}_{k}\right| E_{0}\right\rangle\right|^{2} \delta\left(\omega-E_{0}-E_{n}\right),
\end{aligned}
$$

where $\left|E_{0}\right\rangle$ denotes the ground state with energy $E_{0}$. Using open boundaries (obc), the spin-operator $\mathbf{S}_{k}$ in Fourier space can be defined in the spirit of Ref. [BGJ04] as

$$
\mathbf{S}_{k}=\sqrt{\frac{2}{L+1}} \sum_{j=1}^{L} \sin (j k) \mathbf{S}_{j}
$$

with quasi-momentum $k=l \pi /(L+1), l \in \mathbb{N} \bmod L$. For periodic boundary conditions (pbc) the spin-operator is then given by

$$
\mathbf{S}_{k}=\sqrt{\frac{2}{L}} \sum_{j=1}^{L} \exp (\mathrm{i} j k) \mathbf{S}_{j}
$$

with momentum $k=l \pi / L, l \in \mathbb{Z} \bmod L$. The value of the exchange coupling constant is set to $J=1$ from now on and therefore the ground state is anti-ferromagnetic. Various information of the dynamic structure factor for the anti-ferromagnetic chain in the thermodynamic limit are known from studies of the Bethe ansatz, see Refs. [dCP62, FT81, MBB79, $\left.\mathrm{KMB}^{+} 97, \mathrm{CH} 06\right]$. The eigenstates that lead to non-vanishing weights for the dynamic spin structure factor belong all to the $S=1$ quantum sector [MTBB81]. In the thermodynamic limit the dominant part $(71.30 \%)$ of the structure factor is provided by the 2 -spinon (triplet) contributions, that are located in the interval bounded by Eqn. (5.13). Approximately $27 \%$ of the weight can be devoted to 4 -spinon contributions and the rest $(\approx 2 \%)$ to higher spinon excitations. Also the line shapes for the 2-spinon and 4-spinon contributions have been calculated in the thermodynamic limit, see Refs. $\left[\mathrm{KMB}^{+} 97, \mathrm{CH} 06\right]$. From these studies the dynamic spin structure factor is known to diverge as:

$$
S(k, \omega) \propto\left(\omega-\omega_{1}\right)^{-\frac{1}{2}} \sqrt{\ln \left(1 /\left(\omega-\omega_{1}\right)\right)}
$$

for $k \neq \pi$, respectively

$$
S(\pi, \omega) \propto \omega^{-1} \sqrt{\ln (1 / \omega)}
$$

for $k=\pi$.

\section{\begin{tabular}{l|l} 
Computation with matrix product states & 5.2
\end{tabular}}

The MPS Lanczos method, as introduced in Sec. 3.3.7, is used to calculate the dynamic spin structure factor of the anti-ferromagnetic spin- $\frac{1}{2}$ Heisenberg chain. At first the convergence of the spectral poles/weights with the new reorthogonalization scheme is analysed. Then single spectral poles are compared to results from the Bethe ansatz. 


\section{Implementation and entanglement entropy $\quad 5.2 .1$}

In this work a state of the art MPS code provided by I.P. McCulloch has been used to implement the recursion relation of the Lanczos method. The model has a $S U(2)$ symmetry, which has been exploited in the MPS program [MG02, McC07]. As explained in Sec. 3.3.5 MPS can represent only low-entangled states with high precision. Therefore it would be advantageous if the Lanczos states $\left|f_{n}\right\rangle$ also show only little entanglement. However, the application of the Hamiltonian to the first Lanczos state will increase the entanglement with iteration.

In Fig. 5.2 the entanglement entropy of the Lanczos vectors of the anti-ferromagnetic Heisenbergchain with obc for various system sizes is shown. The entanglement entropy is always calculated for a symmetric partition $A=L / 2$, see Eqn. (3.47). One can clearly see the increase of the entanglement entropy with Lanczos vectors (iterations). The size dependence is not that pronounced. The ground state entanglement entropy is also given for a comparison.

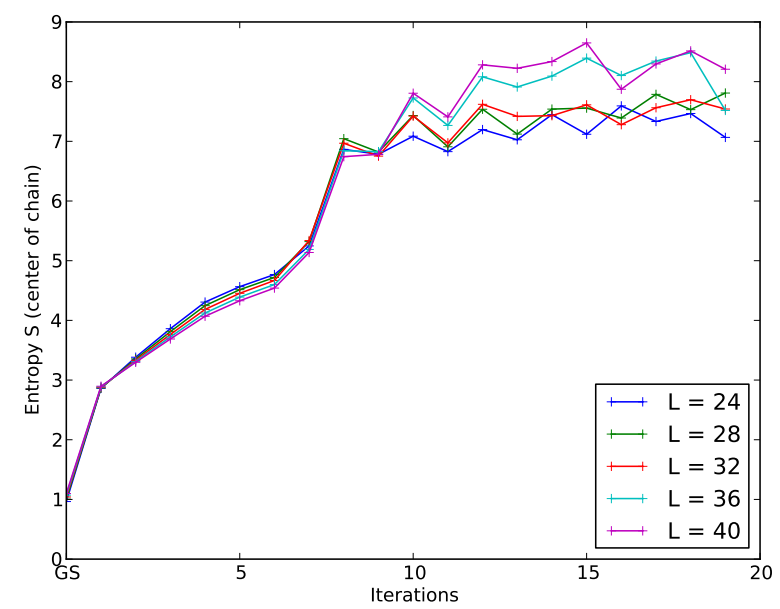

Figure 5.2: Entanglement entropy of the Lanczos states for the anti-ferromagnetic Heisenbergchain with obc for various system sizes. Iteration zero gives the entanglement entropy of the ground state (GS).

The very steep increase of the entanglement entropy will lead to non-optimally represented Lanczos vectors as MPS. This was not unexpected as the Lanczos vectors will have fractions of highly entangled states. However, the non-optimal representation of the Lanczos vectors is the source of the orthogonality losses and the need of the developed reorthogonalization routine as described in Sec. 3.3.7.

\section{Precision and error analysis $\quad 5.2 .2$}

At first the convergence of the spectral poles (eigenenergies) is analysed. In Fig. 5.3 a comparison of the convergence of the spectral weights of the MPS Lanczos method with (right panel) and without (left panel) reorthogonalization is shown, see Sec. 3.3.7. One can clearly see that with a maximal MPS matrix dimension $M=512$ only the first three spectral poles converge without the reorthogonalization. With the suggested reorthogonalization scheme one obtains a much better and more stable convergence, which allows 
to extract already the first 8-9 poles even for this small maximal MPS matrix dimension. Note however, that one of course does not get rid of all the ghosts. Next, the converged
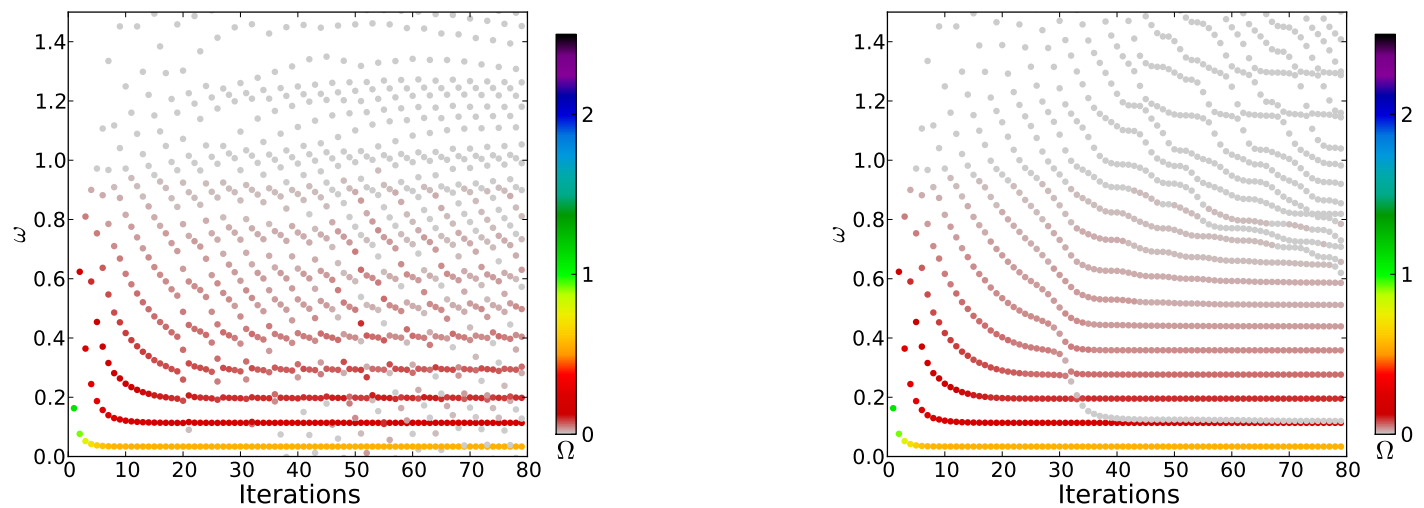

Figure 5.3: (Published in $\left.\left[\mathrm{DWH}^{+} 12\right].\right)$ Comparison of the convergence of the excitation energies and spectral weights for pseudomomentum $k=\pi$ as function of Lanzcos iterations for the spin- $\frac{1}{2}$ Heisenberg chain. The left panel shows the behaviour for the scheme without any reorthogonalization, the right panel the new scheme. Calculations were done for a chain of length $L=32$ (obc), maximal MPS matrix dimension $M=512$. The color coding gives the spectral weight $\Omega$.

spectral poles from pbc calculations are compared to the exact solution from the Bethe ansatz. In Fig. 5.4 the relative error in the position of the first three poles to the exact Bethe ansatz calculations is shown. More precisely, the position of the poles are compared to 2-spinon excitations with lowest energy and $k=\pi$ calculated for periodic boundary conditions in the $\left(S=1, S_{z}=1\right)$ triplet subspace, see Sec. 5.1.2. The error in the ground state $(\mathrm{GS})$ energy is also given.

The weight cutoff in the algorithm was chosen as $\Omega_{c u t}=10^{-3}$ in all cases. The residual cutoff had to be increased with system size from $r_{\text {cut }}=0.1$ for $L=24$ to $r_{\text {cut }}=0.5$ for $L=72$ to be able to reliably extract the spectral weights. As expected one finds that the error in the ground state energy is several orders of magnitude smaller than the error for the excitations. In the left panel of Fig. 5.4 the system size $L$ is varied for a constant maximal MPS matrix dimension $M=512$, while in the right panel the size $L=32$ is fixed and the maximal MPS matrix dimension $M$ is increased. The monotonic decrease of the error shows that it originates chiefly in the approximation of the Lanczos states by MPS.

In summary, the MPS-based Lanczos method (with the suggested reorthogonalization scheme) is able to extract accurate poles and spectral weights for finite chain lengths. The benchmark checks for the energies had to be done for periodic boundary conditions in order to compare to results from the Bethe ansatz. The behaviour of the MPS Lanczos for open boundary conditions is therefore assumed to be even more accurate. However, it will also be limited to a small finite number of poles. 

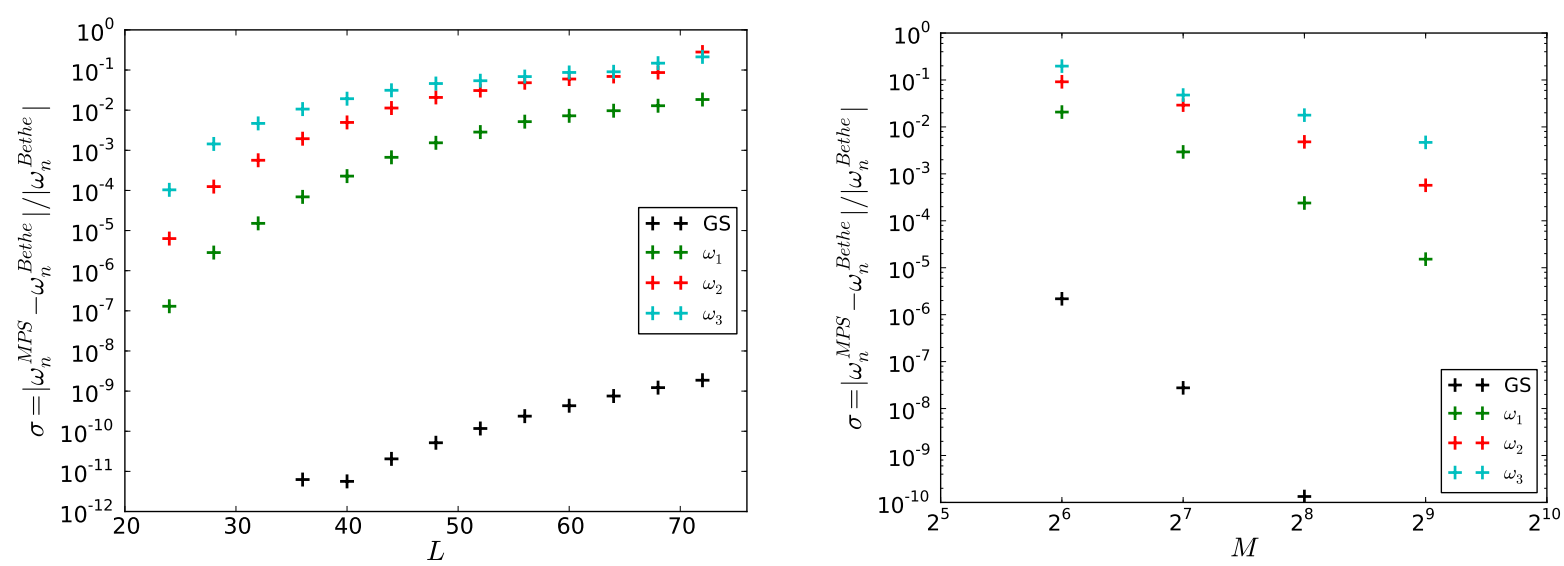

Figure 5.4: (Published in $\left[\mathrm{DWH}^{+} 12\right]$.) The relative error of the first three spectral poles $(k=\pi$, pbc) calculated with the MPS Lanczos method in comparison to the exact 2-spinon excitation energies from the Bethe ansatz. The left panel shows the relative error for different system sizes with a fixed maximal MPS matrix dimension $M=512$. The right panel shows the relative error for different maximal MPS matrix dimension $M$ for a fixed length $L=32$. The error in the ground state (GS) energy is also shown.

\section{Dynamic spin structure factor for $k=\pi \quad 5.2 .3$}

Next, the spin structure factor for $k=\pi$ is calculated for both open and periodic boundary conditions. In order to extrapolate the extracted spectral weights to the thermodynamic limit the rescaling scheme described in Sec. 3.1.2 is used. The results are collected in Fig. 5.5. The energy cutoff for the poles was chosen as $\Omega_{c u t}=10^{-3}$, and the residual cutoff had to be increased with system size to maximal $r_{c u t}=0.5$ to be able to extract the spectral weights. One can clearly see that the spectral weights, rescaled with the scheme discussed in Sec. 3.1.2, nicely collapse onto the results from the 2-spinon contributions of the Bethe ansatz in the thermodynamic limit, ${ }^{2}$ independent of the chosen boundary conditions. Finite-size effects are not very pronounced for $k=\pi$, and all (significant) spectral weights lie within the two-spinon bounds (see Eqn. $(5.13)) \omega_{1}(k=\pi)=0$ to $\omega_{2}(k=\pi)=\pi$.

The position of the second spectral pole differs substantially between open and periodic boundary conditions. Therefore for periodic boundary conditions one would need much longer chains to fill the gap between the first and second spectral poles. The position of the first spectral pole moves very slowly with system size to the origin. Therefore, in order to resolve the divergence for $\omega \rightarrow 0$ one would have to go to much larger system sizes. As stated in Sec. 5.1.3, the eigenstates that lead to non-vanishing weights for the dynamic spin structure factor belong all to the $S=1$ quantum sector. For $k=\pi$ it is the eigenvector with the lowest energy in that subspace that gives the first spectral weight/pole. Therefore an alternative way to check the behaviour for $\omega \rightarrow 0$ for $k=\pi$ is to perform standard ground state DMRG calculations in that subspace. But in order to use the rescaling scheme one needs also the subsequent poles which are not as easy to

\footnotetext{
${ }^{2}$ The Bethe ansatz data is provided by J.S. Caux, see Ref. [CH06].
} 

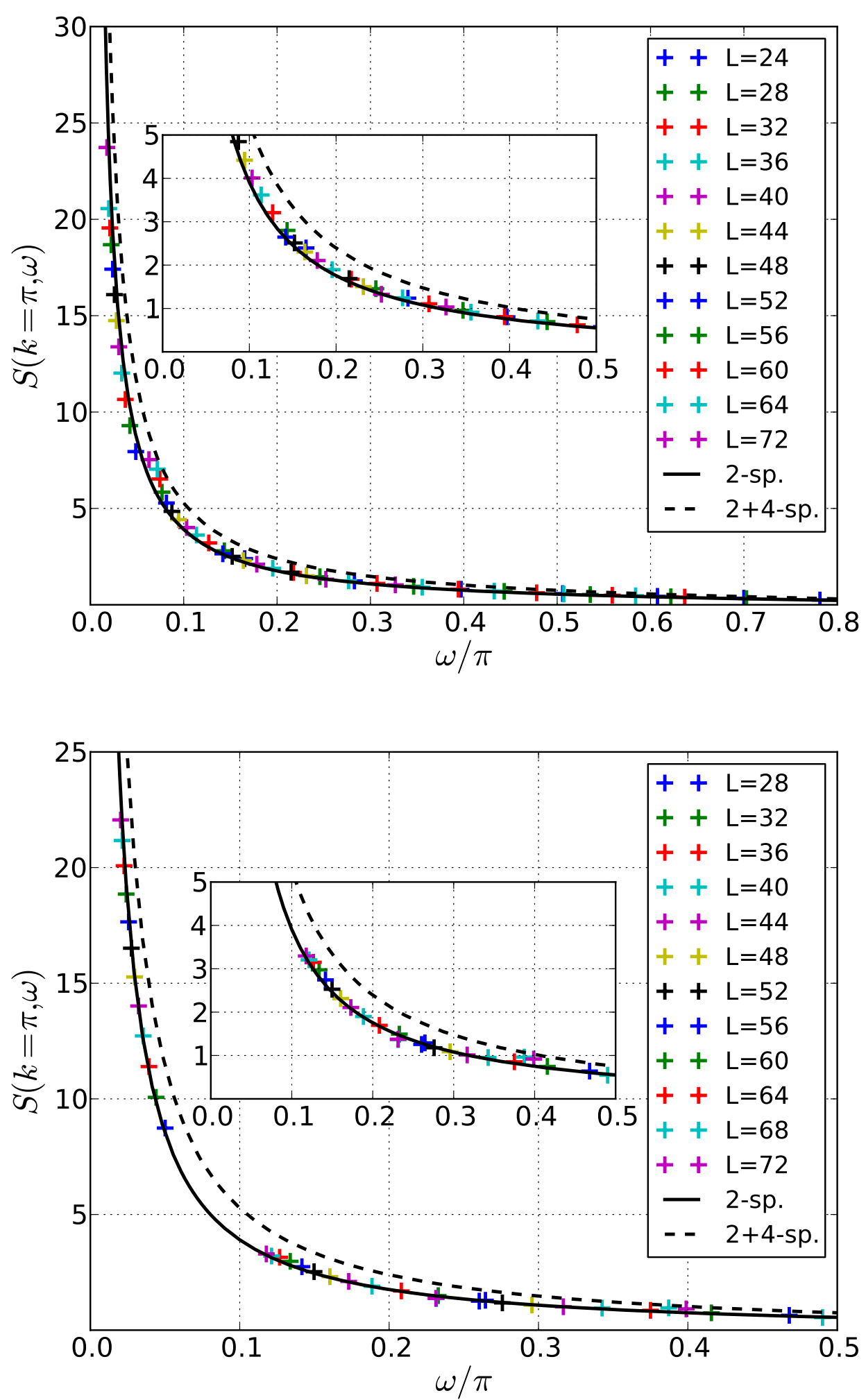

Figure 5.5: (Published in $\left[\mathrm{DWH}^{+} 12\right]$.) Dynamic spin structure factor for $k=\pi$ for open (upper panel) and periodic boundary conditions (lower panel). The spectral weights/poles are chosen by the minimal residual scheme. 
obtain as long as translation symmetry is not implemented. ${ }^{3}$

It turns out that the Lanczos method does not capture the 4-spinon weights (and higher spinon weights). In principle the Lanczos algorithm will also converge towards any multi-spinon state. But for this model the 4-spinon and higher-spinon states show a rather strong finite-size scaling, ${ }^{4}$ which means that 4 -spinon states will appear in the low energy sector only for much longer chains than those considered here. Furthermore, these states will have a small weight in comparison to the 2-spinon states and therefore they are hard to distinguish from ghost states or numerical noise.

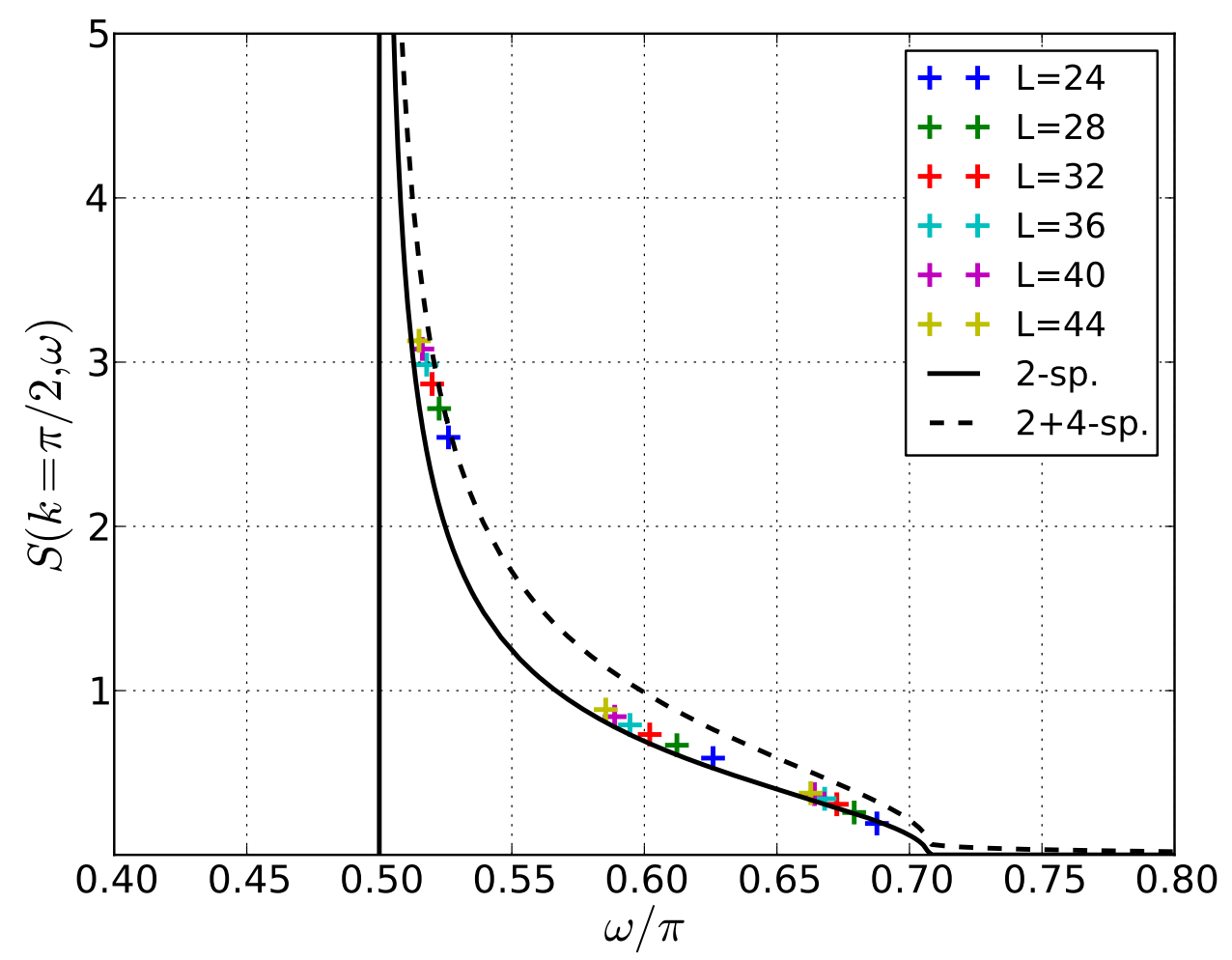

Figure 5.6: (Published in $\left[\mathrm{DWH}^{+} 12\right]$.) Dynamic spin structure factor for $k=\pi / 2$ for periodic boundary conditions and $M=512$. The spectral weights/poles are chosen by the minimal residual scheme.

\footnotetext{
${ }^{3}$ The next eigenvectors that can be obtained by a multi-target approach have no weight for $S(k=\pi, \omega)$ as they have a different momentum. Therefore in order to use the method efficiently one has to search directly in that momentum subspace. That means one has to use translational symmetry and implement momentum as a good quantum number.

${ }^{4}$ For example, the first 4 -spinon pole lies at $\omega=2.41$ for $L=24$, see Ref. [FCC09] and private communication with J.S. Caux.
} 


$$
\begin{array}{l|l}
\text { Dynamic spin structure factor for } k=\frac{\pi}{2} & 5.2 .4
\end{array}
$$

In Fig. 5.6 the dynamic spin structure factor $S\left(k=\frac{\pi}{2}, \omega\right)$ (periodic boundary conditions) calculated with the MPS Lanczos method is shown and compared to the $(2+4)$-spinon contribution obtained from Bethe ansatz [CH06]. The spectral signature starts now in the middle of the spectrum and therefore the extraction of poles and weights is much harder. It was possible to obtain reasonable data up to a length $L=44$ and $M=512$. The low-lying excitations do not collapse as nicely onto the 2-spinon curve as for $k=\pi$. One reason lies in the problematic definition of the first interval in the rescaling scheme, which explicitly depends on the value of the lower bound $\omega_{1}$. It is evident that this value will also be subject to more or less strong finite-size effects. These finite-size effects are obviously even worse for open boundary conditions even though one can go to larger systems.

\section{Divergence analysis $\quad 5.2 .5$}

As mentioned earlier, the structure factor $S(k=\pi, \omega)$ shows a logarithmic correction to the leading divergence as $\omega \rightarrow 0$, see Eqn. (5.18). In order to resolve such an additional feature it is instructive to plot $\omega S(k, \omega)$, which is done in the left upper panel of Fig. 5.7 for periodic boundary conditions. Apparently, the data nicely fall onto the line from the Bethe ansatz, i.e. are in agreement with the predicted logarithmic divergence of this quantity. Note, however, that in order to unambiguously resolve or even predict such a logarithmic divergence one would need to study system sizes well beyond anything possible presently.

In the right upper panel of Fig. 5.7, $\omega S(k=\pi, \omega)$ is plotted again, but for open boundary conditions and with an additional Lorentzian broadening of $\eta=0.1$. For this figure no residual cutoffs or weight cutoffs had to be used as for the extraction of the single weights. The broadening completely smears out the logarithmic divergence, but this is not specific to the method. With a broadening the observation of such a divergence will be nearly impossible. In Figs. 8 and 14 of Ref. [KW99] the same quantity up to a proportionality constant is discussed by Kühner and White. Their Fig. 8 is reproduced here for comparison, Fig. 5.7. Note that due to different schemes of dealing with the open boundary conditions results relate to those in Fig. 5.7 only up to a proportionality constant. The further comparison between the different methods is postponed to Sec. 5.3.

\section{\begin{tabular}{l|l} 
Comparison to other methods & 5.3
\end{tabular}}

The adaptation of the Lanczos algorithm for dynamic correlation functions to the framework of MPS produces accurate results for the spin structure factor. However, this quantity has been chosen to be a (non-trivial) test case and therefore a comparison to other methods is mandatory in the following discussion of the method. 

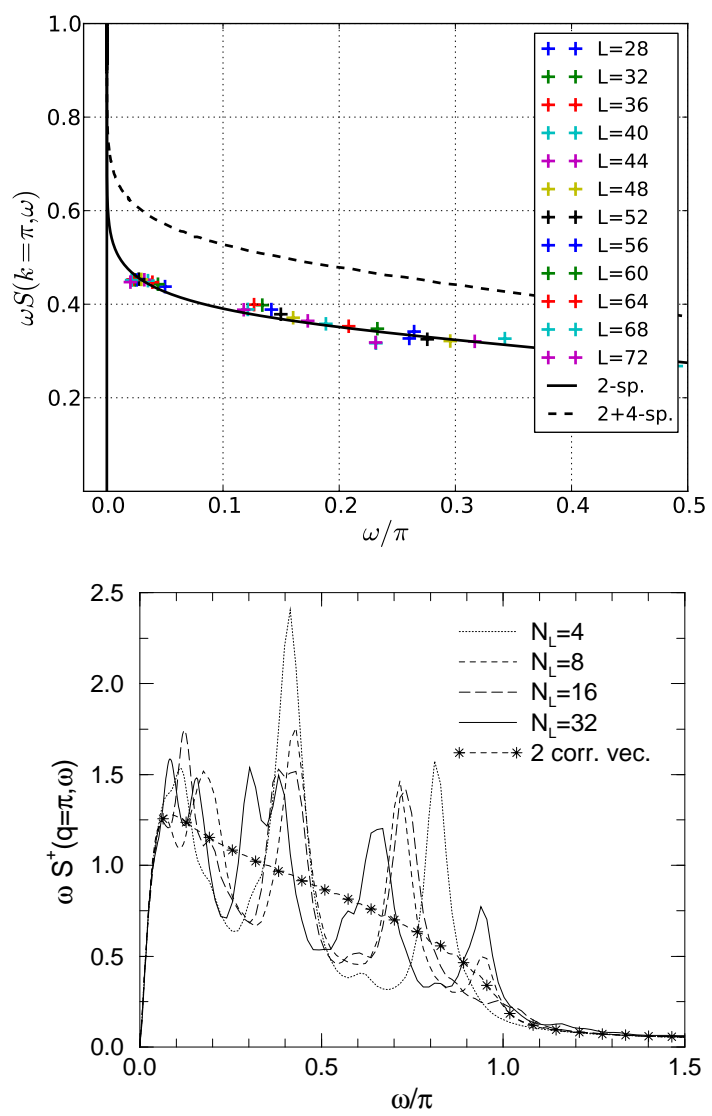

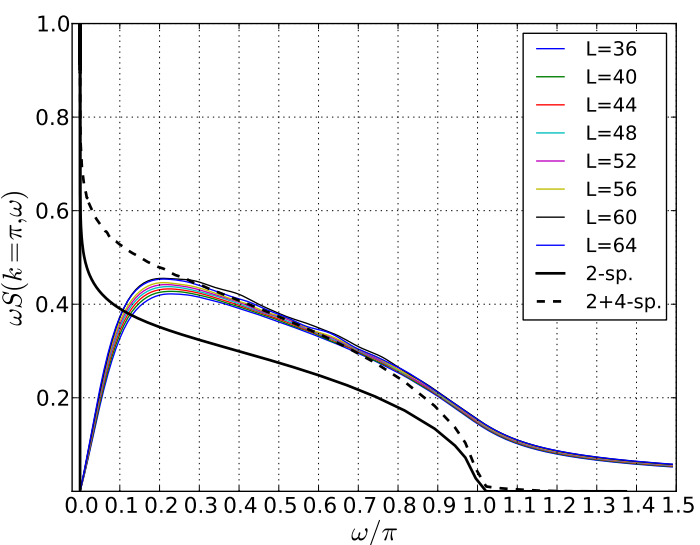

Figure 5.7: Rescaled dynamic spin structure factor $\omega S(k, \omega)$ for $k=\pi$.

upper panel:(Published in $\left[\mathrm{DWH}^{+} 12\right]$.) Data from MPS Lanczos (pbc) based on the same data as in Fig. 5.5 without (left) and with a Lorentzian broadening of $\eta=0.1$ (right).

lower panel: dynamic spin structure factor (obc) $\omega S^{+}(\omega, k)$ for $k=\pi$ with a Lorentzian broadening of $\eta=0.1$, obtained in a standard Lanczos approach (with various numbers of target states $N_{L}$ ) and in the correction vector approach; reproduced from Fig. 8 of Ref. [KW99].

\section{Original Lanczos DMRG method}

As stated in Sec. 3.3.7 the original Lanczos method was the first method for the calculation of spectral functions. In Fig. 5.7 the spin structure factor calculated by Kühner et al. with the standard DMRG Lanczos method and additional broadening as described in Sec. 3.3.7 is shown. Although different schemes for dealing with the open boundary conditions preclude a quantitative comparison, their results clearly show that the standard Lanczos DMRG is not capable of producing a smooth, converged curve consistent with the predictions from Bethe ansatz, but display substantial artificial structure. This comparison clearly shows that with the new MPS Lanczos algorithm one has reached a definite improvement over the standard Lanczos DMRG method.

\section{\begin{tabular}{l|l} 
Correction vector & 5.3 .2
\end{tabular}}

The correction vector method has established itself as the standard method for the calculation of spectral functions. In Fig. 5.7 the spin structure factor calculated by Kühner et al. with the correction vector as described in Sec. 3.3.7 is shown. The overall line shape of the dynamic spin structure factor is similar. However, one should emphasize that the inherent broadening of the correction vector completely annihilates any signature of the 
divergence for $\omega \rightarrow 0$, see Fig. 14, Ref. [KW99]. For every frequency point and every broadening a new correction vector has to be calculated, which makes the calculation of a high-resolution spectral function very costly, especially with decreasing broadening. However, the correction vector gives the overall (broadened) line shape very reliable.

\section{\begin{tabular}{l|l} 
Chebyshev polynomials & 5.3 .3
\end{tabular}}

In a recent work the dynamic spin structure factor for the anti-ferromagnetic spin- $\frac{1}{2}$ Heisenberg chain was calculated with an expansion in Chebyshev polynomials [HWM $\left.{ }^{+} 11\right]$. The algorithm has been briefly introduced in Sec. 3.3.7. In Fig. 5.8 the dynamic spin structure factor for $k=\pi$ and open boundary conditions with the expansion in Chebyshev polynomials by Holzner et al. $\left[\mathrm{HWM}^{+} 11\right]$ is shown.

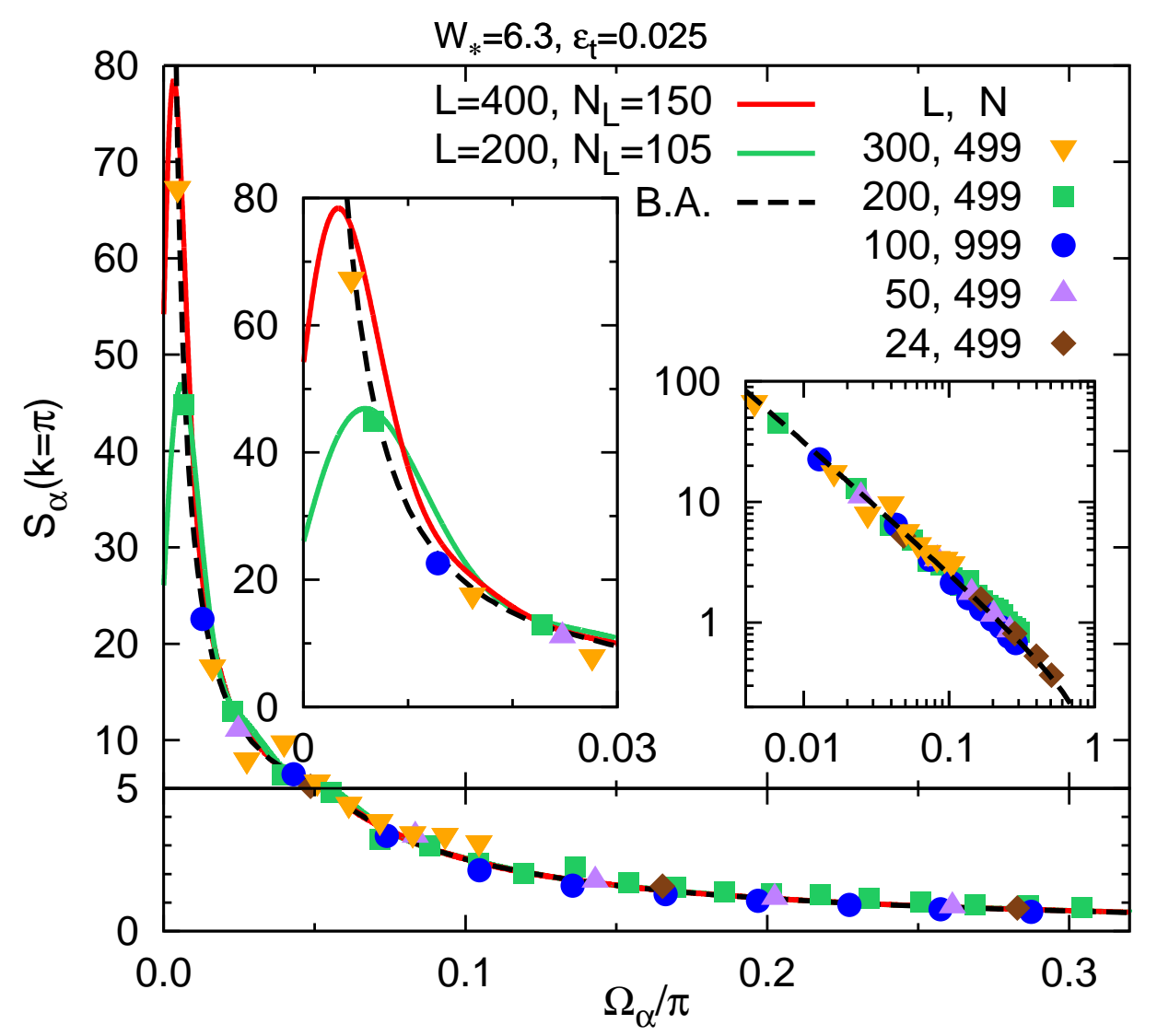

Figure 5.8: Dynamic spin structure factor for $k=\pi$ and open boundary conditions with Chebyshev polynomials, reproduced from Fig. 7 of Ref. [HWM $\left.{ }^{+} 11\right]$.

Due to a special energy truncation scheme the algorithm allows to calculate very long chains. For $L=400$ and $L=200$ the continuous line shapes nicely resemble the data of the Bethe ansatz. ${ }^{5}$ However, in the inset one can see clear deviations in the low energy spectrum due to the finite chains. They were also able to extract discrete spectral

\footnotetext{
${ }^{5}$ It is assumed that this data corresponds to the $(2+4)$-spinon contributions.
} 
weights from their continous line shapes that are shown as dots in the figure. This was accomplished by increasing the number of expansion paramters to $N=499(999)$. Then the spectral function shows peaks at the position of the spectral poles which were fitted to extract their weight and position. The used rescaling scheme for the single spectral weights is identical to the one used in this work. The single spectral weights also lie on the curve of the Bethe ansatz. However, this is questionable as they should correspond to the 2-spinon excitations only.

Due to the similar calculations scheme (similar recursion formula) the Chebyshev polynomials can be regarded as the main competitor of the newly developed MPS Lanczos approach. In particular, as it has basically the same (low) numerical costs. Compared to each other, both have advantages and disadvantages, such that no conclusive statement can be made at the moment, also in view of the lack of applications so far. Both rely on operations $H|\psi\rangle$ carried out a similar number of times, but the Chebyshev approach can handle longer chains $(\approx 400$ sites) because of a surprising low maximal MPS matrix dimension $(M \sim 32-64)$; this is arguably due to an inherent entanglement-reducing energy-truncation scheme. However, comparing directly the evaluation of discrete spectral weights/poles, one has to perform many iterations $(500-1000)$ within the Chebyshev expansion. Furthermore, one has to fit the broadened spectral weights to get the discrete poles/weights including a fit error. This becomes even more challenging for dense spectral poles, where the broadened peaks overlap. This problem could be seen at the test case of the spin- $\frac{1}{2}$ Heisenberg chain where the extracted weights had too much weight compared to the expectations for the 2-spinon excitations. Generally, broadening is an inherent part of the Chebyshev approach. It also has more tuning parameters that have to be optimized for the method to work properly, especially the number of iterations is somehow arbitrary.

\section{\begin{tabular}{l|l} 
Other algorithms & 5.3 .4
\end{tabular}}

The Fourier transform of time-dependent DMRG data as described in Sec. 3.3.7 is another method for calculating spectral functions. It has been applied by Pereira et al. [PWA08, PWA09] to the dynamic spin structure factor of the spin- $\frac{1}{2}$-Heisenbergchain. They were also able to resolve the divergence, however the low frequency behaviour is especially vulnerable to the extrapolation of the finite-time data. Some other algorithms for time evolution of quantum states also use a Krylov space representation in MPS [Sch04, NM05, MMN05, GR06, $\mathrm{KKM}^{+} 08, \mathrm{FCM}^{+}$08]. Here the proposed reorthogonalization may also be useful.

Recently, two algorithms for calculating spectra of translationally invariant Hamiltonians with (unitary) MPS working directly in the thermodynamic limit and with periodic boundary conditions $\left[\mathrm{HPW}^{+} 12, \mathrm{PHV} 12\right]$ were suggested that can approximate many branches of the dispersion relation with high precision. It would be really interesting to see whether these algorithms can also calculate dynamic correlation functions with that precision and how they compare to the Lanczos expansion. 


\section{Conclusion

"Remember that all models are wrong; the practical question is how wrong do they have to be to not be useful."

(Book: "Empirical Model-Building and Response Surfaces", Box and Draper, 1987,[BD87])

The two different topics of this thesis are discussed separately. The results are briefly summarized and critically reviewed. Furthermore, new perspectives are opened up.

\section{\begin{tabular}{l||l} 
Long range Kondo signatures & 6.1
\end{tabular}}

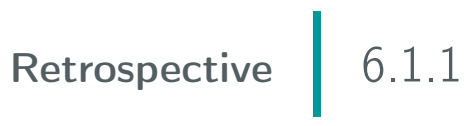

The task of this project was to find a theoretical model for the experimental STS data of buried magnetic impurities, to calculate the LDOS for this model at the surface for impurities in different depth and to compare the results to the experiment.

To incorporate the strong interactions in the impurities which lead to the Kondo effect the SIAM was chosen. In order to include also the focussing effect of the copper crystal the LCAO method was used for the calculation of the energy dispersion of the conduction band electrons needed in the SIAM. The LDOS at the impurity for this model was then calculated with the NRG. Using the LDOS at the impurity and the LCAO Green's functions of the copper crystal the LDOS at the surface was calculated via the equation of motion technique.

Simulation and experiment showed very good agreement in the single spectra, but also in the general spectral maps for various impurities at different depth below the surface. In order to extract the new physics three major questions have been answered in Sec. 4.6:

1. Is the chosen effective model appropriate for the experimental data?

2. Did we observe a real long range Kondo signature?

3. Did we measure the Kondo cloud?

Concerning the first question, it was stated that the chosen effective model is one of the simplest models that shows the Kondo effect. Especially the basic modelling of the degrees of freedom of the impurity is a big simplification. However, the Kondo resonance in the spectral function is known to be a universal effect that is to a large extend rather robust with respect to impurity details. In Sec. 4.6.1 different more realistic models, that 
incorporate the inner structure of the impurity atom in more detail, were discussed. The known results from the literature are summarized, showing that in most cases our chosen effective model is justified. Nevertheless, this study revealed that the extracted numbers for the Kondo temperature should be interpreted with care. They should be interpreted as an 'effective Kondo temperature' related to the SIAM. One advantage of our chosen model is the limited number of parameters. More realistic models are usually accompanied by a larger number of parameters, which are usually unknown and hard to derive from bandstructure calculations like DFT or LCAO. Summarized, it can be stated that the SIAM together with the LCAO bandstructure calculations are a good choice for the modelling of the measured data.

The second question was also answered positively: Many signs support the finding of a long range Kondo signature. The strong signal at the Fermi energy, the phase shift smaller than $\pi / 2$, the relation between the 'effective' Kondo temperatures for Fe and Co, the oscillations with depth due to the surface of the crystal - all these elements fit into the picture of a long range Kondo signature observed at the surface.

The third question had to be answered negatively: The Kondo cloud was not measured. Following Affleck [Aff09] the Kondo cloud can not be measured from the Green's function directly. Only the integrated Green's function leading to the charge density will give an access to the Kondo cloud. In order to observe the Kondo cloud form this quantity signatures from much more distant magnetic impurities have to be recorded. This will probably be out of reach within this experimental setup at least in the near future.

\section{\begin{tabular}{l|l} 
Perspective & 6.1 .2
\end{tabular}}

The successful observation of a long range Kondo signature opens new perspectives. Two main routes are possible. At first further characteristics of the Kondo effect for single impurities can be examined. Then, in a second step, more complex impurity systems can be investigated.

Additional experiments could reveal further characteristics of the Kondo effect. In an applied magnetic field a splitting of the Kondo resonance should be observed. This would further underline the existence of a long range Kondo signature. Considering available magnetic fields, manganese may be a possible candidate. For adsorbed atoms this splitting was recently shown, see Ref. $\left[\mathrm{OTvB}^{+} 08\right]$. Comparing many different impurities in various host materials even more intrinsic properties of the impurities may be analysed, e.g. the effect of Hund's coupling to the Kondo temperature. However, this would demand much more data from the experimental side and also the theoretical models have to be refined to incorporate these effects. Spin-resolved STM measurements are also a very promising approach to reveal new characteristics from buried magnetic impurities. Maybe with these even spin-spin correlation functions can be measured which would give a very direct access to the Kondo cloud. 
A very interesting field of Kondo physics is the interplay between two magnetic impurities. In the simplest picture these can be modelled by two single local moments $\mathbf{S}_{i}$ at two different positions $i=1,2$ in distance $R$, that interact via an exchange coupling $J$ with the spins of the conduction band electrons. In first-order perturbation theory an effective Hamiltonian was derived by Ruderman, Kittel, Kasuya and Yosida (RKKY) [RK54, Kas56, Yos57b]:

$$
H_{R K K Y}=J \frac{\sin \left(2 k_{f} R\right)}{R^{d}} \mathbf{S}_{1} \cdot \mathbf{S}_{2}
$$

Depending on the distance $R$ this effective coupling between the two local moments can be anti-ferromagnetic or ferromagnetic. This, so called RKKY interaction, will compete with the Kondo interaction: The Kondo effect will try to screen the impurity spins from each other (vanishing spin-spin correlations between the two impurity spins). The RKKY interaction favors the formation of strong correlations between the impurity spins (strong spin-spin correlations between the two impurity spins). Reported first measurement of two buried magnetic impurities close to each other show differences in the LDOS at the surface. This is a promising approach to understand and measure the interplay between the two effects. A theoretical modelling of this system is far more complex. A two-impurity Anderson model with an effective RKKY interaction $J$ between the impurities can give a first explanation for the impurity spectral functions [JKmW81, JV87, JVW88, JV89]. Here the effect of the interplay between RKKY and Kondo interaction to the impurity spectral function can be studied with the NRG. However, to calculate the LDOS at the surface including the focussing effect of the copper crystal will be a big challenge.

The deeper understanding of Kondo physics and the interplay with the RKKY interaction is also very important for the so-called heavy fermion systems which can show unconventional superconductivity [AGO75, SAB ${ }^{+} 79$, Ste84]. The systems are characterized by conduction electrons that behave like free electrons with an enhanced free-electron mass (up to 1000 times the free-electron mass). The materials are usually intermetallic compounds containing elements which have a partially filled $4 f$ or $5 f$-shell, see introduction Sec. 1.1. Typical compounds are e.g. CeCuSi $i_{2} \mathrm{CeCu}_{6}, \mathrm{UBe}_{13}$. The electrons in the $f$-state are highly localized and form due to Hund's rules a local magnetic moment like the magnetic impurities regarded in this work. However, in heavy fermion systems these local moments are formed at every atomic site. Therefore a Kondo lattice model with a local moment at every site that interacts via an exchange interaction with the conduction electrons is the simplest model for these systems [Col12]. For strong effective RKKY interactions between the local moments a magnetic state will develop. For weak interactions the conduction electrons can screen the local moments via the Kondo effect leading to a paramagnetic state. Between these to regimes a quantum critical point occurs and superconductivity may appear. However, the screening of a local moment by the conduction electrons via the Kondo effect leads to the Kondo screening cloud, see Sec. 4.2.2. For heavy fermion system it would extend over hundreds of lattice constants. Therefore for dense systems interference of these Kondo clouds would occur which has not been seen experimentally by now. Furthermore the so-called exhaustion problem can occur: There would not be enough low-energy conduction electrons to screen the many 
local moments [Col12]. Therefore, a deeper understanding of the spatial behaviour of the Kondo effect is also of great interest for the heavy fermion systems.

\section{Dynamic spin structure factor of the one-dimensional Heisenberg model

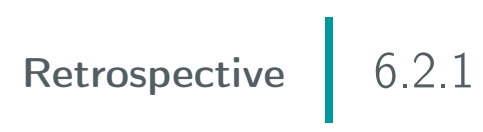

In this project a new algorithm for the calculation of spectral functions with DMRG/MPS was developed. It is based on previous ideas to implement the Lanczos method in the DMRG framework. In this new method the Lanczos algorithm is directly implemented in MPS. As a non-trivial test case, the dynamic spin structure factor of the one-dimensional Heisenberg model was calculated.

It was shown that the Lanczos algorithm in MPS formulation is capable of calculating a number of discrete spectral weights/poles for spin chains that are longer than those that can be evaluated with exact diagonalization and the standard Lanczos algorithm. A comparison with the exact eigenenergies obtained from the Bethe ansatz showed that the low lying spectral poles can be obtained with good accuracy. The form of the dynamic spin structure factor for the 2-spinon contribution from the Bethe ansatz in the thermodynamic limit could be convincingly reproduced with the rescaling scheme. Higher spinon-contributions to the dynamic spin structure factor could not be extracted from the data due to their very low spectral weight and strong finite-size scaling.

The main discrepancy between the excitation energies obtained from the Bethe ansatz and the ones obtained from the MPS Lanczos method was shown to be originated in the approximation of the Lanczos states via MPS. A bad approximation of the Lanczos states is reasoned in the limited entanglement entropy that can be represented with MPS. The repetitive application of the Hamiltonian to the Lanczos states increases the entanglement entropy and therefore the quality of the representation of the states by MPS decreases with each iteration. The main limitation to apply the method to longer chains is thus originated in the increasing entanglement entropy as for long chains many iterations are needed to get all relevant spectral poles and weights. The increase of the entanglement entropy in the Lanczos states due to the repetitive application of the Hamiltonian will however occur for all methods that try to setup a Krylov space.

The error due to the approximation of the Lanczos states by MPS leads to a loss of orthogonality between the Lanczos vectors. This loss of orthogonality can be cured by reorthogonalization schemes. In this work it was realized that the standard reorthogonalization schemes are not successful and therefore a new way to restore reorthogonalization was developed. The proposed reorthogonalization scheme which leads to the generalized eigenvalue problem instead of a tridiagonal matrix can cure this loss of orthogonalization to a certain extend. 
The newly developed method had to be benchmarked to existing methods. At first it was compared to the original and adaptive DMRG Lanczos method. The broadly distributed spectral weight of the $S=\frac{1}{2}$ Heisenberg antiferromagnet had been a major stumbling block for the original DMRG-based formulation. In technical terms, the new algorithm offers the following advantages over the original and adaptive DMRG Lanczos method: The algorithm can be easily implemented in any MPS algorithm as it only uses simple MPS algebra routines. Multi-targeting and the inexact Hamiltonian representation is avoided. Furthermore the possibility of handling the Lanczos states individually by a single MPS allows for reorthogonalization procedures that are not possible with the adaptive Lanczos method. This reorthogonalization significantly improves the convergence of the spectral poles and makes the extraction of single weights much easier. Therefore it represents a definite improvement over existing Lanczos methods in the DMRG framework.

In comparison to other non-Lanczos methods the MPS Lanczos method appears neither as clearly superior nor as inferior. In comparison to the correction vector, Chebyshev polynomials and Fourier transform of time-dependent DMRG data its relative performance will be strongly dependent on the chosen model and the type of the spectral function. Spectral functions, that are formed by many excitations e.g. the LDOS in the Hubbard band will be difficult to calculate with the MPS Lanczos method due to the large amount of spectral poles with a non-vanishing spectral weight. This was shown in a very recent work [Tie12] where the developed Lanczos MPS was applied to the onedimensional Hubbard model. It could be shown that the MPS Lanczos method is not able to resolve the LDOS and the optical conductivity due to the many spectral poles. The correction vector turned out to be clearly superior for this model and these kinds of spectral functions. However, the Lanczos MPS method was useful to determine the optical gap.

\section{\begin{tabular}{l|l} 
Perspective & 6.2 .2
\end{tabular}}

The newly developed MPS Lanczos method has been proven to be successful for the calculation of the dynamic spin structure factor for the $S=\frac{1}{2}$ Heisenberg antiferromagnet - a non-trivial test case for MPS. However, the recent drawback of the method for the application to spectral functions of the one-dimensional Hubbard model has to mentioned [Tie12]. Therefore I believe that the MPS Lanczos will show its full potential for models with a very limited number of discrete spectral poles, like the spin- $\frac{1}{2}$ Heisenberg chain. For those kinds of spectral functions it is nearly impossible to get valuable information for the behaviour in the thermodynamic limit from one single chain in the available length scales. One should emphasize that all the information about the spectral function for finite systems is given exclusively by these discrete spectral weights/poles and therefore any method for the DMRG/MPS that gives a continuous function (e.g. correction vector, expansion in Chebyshev polynomials, Lanczos plus continuous fraction) only interpolates between these points depending on the broadening scheme of the used method. The used rescaling scheme based on the explicit extraction of discrete spectral weights and 
poles gives a very controlled access to the problematic limit $\eta \rightarrow 0, L \rightarrow \infty$ and by this gives more transparent results without broadening. This is especially useful for spectral functions that show divergences as the dynamic spin structure factor where an additional broadening completely smears out the divergence.

Therefore I speculate that applications of the method to other spin models promises to provide spectral functions with good resolution. This is very important for (quasi) one-dimensional materials that can be measured with neutron scattering. Some of these systems can be modelled by spin models with (anisotropic) long-range interactions, for an example see Ref. [ $\left.\mathrm{JOK}^{+} 11\right]$. These models are in most cases non-integrable and therefore no solution with the Bethe ansatz exists and numerical methods are mandatory. Here the developed MPS Lanczos method - especially due to missing broadening - is promising to provide valuable information about spectral gaps and possible divergences.

The developed MPS Lanczos algorithms is (so far) the final step in the history of DMRG/MPS Lanczos algorithms. With this algorithm every Lanczos state is represented by a single MPS with an exact Hamiltonian representation by a MPO. It is therefore the direct implementation of the Lanczos algorithm for this class of variational states and there seems to be no more possible improvement of the implementation of the Lanczos method in MPS. The main limitation is the increasing entanglement entropy of the Lanczos states which is problematic for a MPS representation. Thus MPS as variational states do not seem to be optimal for the implementation of the Lanczos algorithm. Variational states that are capable of representing higher entanglement entropy should be superior. Nevertheless, at the moment due to the lack of alternatives MPS are the most successful variational states that can be used within the Lanczos algorithm for one-dimensional systems. However, the fast and numerous activities in this field may provide a new class of variational states which can efficiently represent (higher) entangled states and therefore be promising for a use as variational states within the Lanczos algorithm. Especially, variational states that can somehow automatically incorporate orthogonality within the Lanczos recursion would be favourable.

Another possible application of the Lanczos method in the (far) future may lie within the field of the newly developed tensor networks for higher dimensions. The Lanczos method just needs basic algebraic operations, like the application of the Hamiltonian to a state, the addition of several states and the evaluation of expectation values. If these operations can be computed with reasonable computational efficiency within these tensor networks, the Lanczos method may give an access to spectral functions even in higher dimensions. 
Appendices 



\section{Computational details}

\section{Matrix product states}

A.1

\section{Matrix product state representation of quantum states}

For a given quantum state a MPS representation can be given exactly. In order to get an exact MPS representation for a quantum state with coefficients $c_{\sigma_{1} \ldots \sigma_{L}}$

$$
|\psi\rangle=\sum_{\sigma_{1}, \ldots, \sigma_{L}} c_{\sigma_{1} \ldots \sigma_{L}}\left|\sigma_{1}, \ldots, \sigma_{L}\right\rangle=\sum_{\sigma_{1}, \ldots, \sigma_{L}} A^{\sigma_{1}} \cdot \ldots \cdot A^{\sigma_{L}}\left|\sigma_{1}, \ldots, \sigma_{L}\right\rangle
$$

one has to reshuffle $2^{L}$ coefficients into a matrix $c^{\prime}$ of dimension $2 \times 2^{L-1}$ :

$$
c_{\sigma_{1} \ldots \sigma_{L}}=c_{\sigma_{1},\left(\sigma_{2} \ldots \sigma_{L}\right)}^{\prime} .
$$

Applying a singular value decomposition (SVD) on the matrix $c^{\prime}$ leads to

$$
c_{\sigma_{1},\left(\sigma_{2} \ldots \sigma_{L}\right)}^{\prime}=\sum_{a_{1}}^{r_{1}} U_{\sigma_{1}, a_{1}} S_{a_{1}, a_{1}}\left(V^{\dagger}\right)_{a_{1},\left(\sigma_{2} \ldots \sigma_{L}\right)} .
$$

From $S$ and $V^{\dagger}$ a new $c_{a_{1} \sigma_{2} \ldots \sigma_{L}}^{\prime}$ can be generated, that will be reshuffled into a matrix $c_{\left(a_{1} \sigma_{2}\right),\left(\sigma_{3} \ldots \sigma_{L}\right)}^{\prime \prime}$. Furthermore the matrix $U$ (with $U^{\dagger} U=1$ ) will be reshuffled into two $A^{\sigma_{1}}$-matrices, with coefficients: $A_{1, a_{1}}^{\sigma_{1}}=U_{\sigma_{1}, a_{1}}$. One obtains:

$$
c_{\sigma_{1}, \ldots \sigma_{L}}=\sum_{a_{1}} A_{1, a_{1}}^{\sigma_{1}} c_{\left(a_{1} \sigma_{2}\right),\left(\sigma_{3} \ldots \sigma_{L}\right)}^{\prime \prime}
$$

One continues with an SVD on $c_{\left(a_{1} \sigma_{2}\right),\left(\sigma_{3} \ldots \sigma_{L}\right)}^{\prime \prime}$. The new $S$ and $V^{\dagger}$ will again be combined and reshuffled into two $A$-matrices. The new coefficients are now: $A_{a_{1}, a_{2}}^{\sigma_{2}}=U_{\left(a_{1} \sigma_{2}\right), a_{2}}$. This procedure is repeated untill site $L-1$ is reached. One finally obtains:

$$
\begin{aligned}
c_{\sigma_{1}, \ldots \sigma_{L}} & =\sum_{a_{1}} \cdots \sum_{a_{l-1}} A_{1, a_{1}}^{\sigma_{1}} \cdots A_{a_{l-2}, a_{l-1}}^{\sigma_{l-1}} \underbrace{c_{\left(a_{l-1} \sigma_{l}\right),\left(\sigma_{l+1} \ldots \sigma_{L}\right)}^{\prime \cdots \prime}}_{M_{a_{L-1}, a_{L}}^{\sigma_{L}}} \\
& =A^{\sigma_{1}} \cdots A^{\sigma_{L-1}} M^{\sigma_{L}} .
\end{aligned}
$$

The $A$-matrices fullfil the condition for left-normalization $\sum_{\sigma_{l}} A^{\sigma_{l}^{\dagger}} A^{\sigma_{l}}=1$. 


\section{Hamiltonian as a Matrix Product Operator}

The explicit construction of the Hamiltonian as a MPO was at first presented by McCulloch [McC07]. Further developments can be found in Ref. [PMCV10] and generalizations for Hamiltonians with long-range interactions in Ref. [FND10]. The following short description on the construction of a MPO is presented using a general short-range spin-Hamiltonian such as:

$$
\hat{H}=\sum_{i=1}^{L-1} \frac{J}{2} \hat{S}_{i}^{+} \hat{S}_{i+1}^{-}+\frac{J}{2} \hat{S}_{i}^{-} \hat{S}_{i+1}^{+}+J^{z} \hat{S}_{i}^{z} \hat{S}_{i+1}^{z}-h_{z} \sum_{i}^{L} \hat{S}_{i}^{z}-h_{x} \sum_{i}^{L} \hat{S}_{i}^{x} .
$$

In order to construct the MPO the tensor structure of the Hamiltonian can be exploited:

$$
\hat{H}=J^{z} \hat{S}_{1}^{z} \otimes \hat{S}_{2}^{z} \otimes \hat{I}_{3} \otimes \hat{I}_{4} \ldots+\hat{I}_{1} \otimes J^{z} \hat{S}_{2}^{z} \otimes \hat{S}_{3}^{z} \otimes \hat{I}_{4} \ldots+\ldots
$$

To construct the Hamiltonian the $W$-matrices (See Eqn. 3.34) have to be defined:

$$
\hat{W}^{[i]}=\sum_{\sigma_{i}, \sigma_{i}^{\prime}} W^{\sigma_{i} \sigma_{i}^{\prime}}\left|\sigma_{i}\right\rangle\left\langle\sigma_{i}^{\prime}\right|
$$

In order to fulfil the tensor structure in Eqn. A.7 they can be determined to be

$$
\hat{W}^{[i]}=\left(\begin{array}{ccccc}
\hat{I} & 0 & 0 & 0 & 0 \\
\hat{S}^{+} & 0 & 0 & 0 & 0 \\
\hat{S}^{-} & 0 & 0 & 0 & 0 \\
\hat{S}^{z} & 0 & 0 & 0 & 0 \\
-h_{z} \hat{S}^{z}-h_{x} \hat{S}^{x} & (J / 2) \hat{S}^{-} & (J / 2) \hat{S}^{+} & J^{z} \hat{S}^{z} & \hat{I}
\end{array}\right)
$$

whereby the first and last matrix is a vector given by:

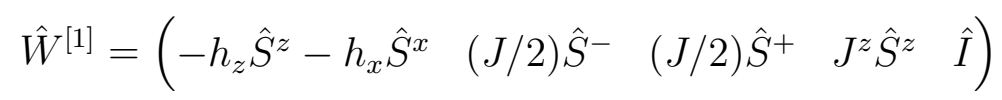

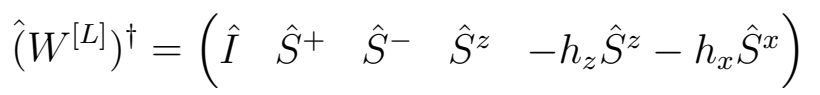

and the $W^{\sigma_{i}, \sigma_{i}^{\prime}}$ can be directly constructed from the physical operators:

$$
\hat{W}_{j, k}^{\sigma_{i} \sigma_{i}^{\prime}}=\left\langle\sigma_{i}\left|\hat{W}_{j, k}^{[i]}\right| \sigma_{i}^{\prime}\right\rangle
$$

\section{DMRG ground state search}

\section{Preparing steps}

In order to use the local DMRG optimization, the expectation value $\langle\psi|\hat{H}| \psi\rangle$ has to be represented in a compact form for the matrix at site $l$. Using the mixed-canonical MPS 
representation of a state: $|\psi\rangle=A^{\sigma_{1}^{\prime}} \cdots A^{\sigma_{l-1}^{\prime}} M^{\sigma_{l}^{\prime}} B^{\sigma_{l+1}^{\prime}} \cdots B^{\sigma_{L}^{\prime}}\left|\boldsymbol{\sigma}^{\prime}\right\rangle$ the expectation can be written as

$$
\begin{aligned}
& \langle\psi|\hat{H}| \psi\rangle=\sum_{\boldsymbol{\sigma}, \boldsymbol{\sigma}^{\prime}}\left\langle\boldsymbol{\sigma}|\underbrace{B^{\sigma_{L} *} \cdots B^{\sigma_{l+1} *} M^{\sigma_{l} *} A^{\sigma_{l-1} *} \cdots A^{\sigma_{1} *}}_{B^{\sigma_{L}^{*} \cdots A^{\sigma_{1}}}} \hat{H} \underbrace{A^{\sigma_{1}^{\prime}} \cdots A^{\sigma_{l-1}^{\prime}} M^{\sigma_{l}^{\prime}} B^{\sigma_{l+1}^{\prime}} \cdots B^{\sigma_{L}^{\prime}}}_{A^{\sigma_{1}^{\prime} \cdots B^{\sigma_{L}^{\prime}}}}| \boldsymbol{\sigma}^{\prime}\right\rangle \\
& =\sum_{\boldsymbol{\sigma}, \boldsymbol{\sigma}^{\prime}} \sum_{\tilde{\boldsymbol{\sigma}}, \tilde{\boldsymbol{\sigma}}^{\prime}}\left\langle\boldsymbol{\sigma}\left|B^{\sigma_{L} *} \cdots A^{\sigma_{1} *}\right| \tilde{\boldsymbol{\sigma}}\right\rangle W^{\tilde{\sigma}_{1}, \tilde{\sigma}_{1}^{\prime}} \cdots W^{\tilde{\sigma}_{L}, \tilde{\sigma}_{L}^{\prime}}\left\langle\tilde{\boldsymbol{\sigma}}^{\prime}\left|A^{\sigma_{1}^{\prime}} \cdots B^{\sigma_{L}^{\prime}}\right| \boldsymbol{\sigma}^{\prime}\right\rangle \\
& =\sum_{\boldsymbol{\sigma}, \boldsymbol{\sigma}^{\prime}} \sum_{\tilde{\boldsymbol{\sigma}}, \tilde{\boldsymbol{\sigma}}^{\prime}} \underbrace{\langle\boldsymbol{\sigma} \mid \tilde{\boldsymbol{\sigma}}\rangle}_{\delta_{\sigma, \tilde{\sigma}}} \sum_{a_{i}, a_{i}^{\prime}, b_{i}} B_{b_{L-1}, 1}^{\sigma_{L} *} \cdots A_{1, a_{1}}^{\sigma_{1} *} W_{1, b_{1}}^{\tilde{\sigma}_{1}, \tilde{\sigma}_{1}^{\prime}} \cdots W_{b_{L-1}, 1}^{\tilde{\sigma}_{L}, \tilde{\sigma}_{L}^{\prime}} A_{1, a_{1}^{\prime}}^{\sigma_{1}^{\prime}} \cdots B_{a_{L-1}^{\prime}, 1}^{\sigma_{L}^{\prime}} \underbrace{\left\langle\tilde{\boldsymbol{\sigma}}^{\prime} \mid \boldsymbol{\sigma}^{\prime}\right\rangle}_{\delta_{\sigma^{\prime}, \tilde{\sigma}^{\prime}}} \\
& =\sum_{a_{l-1}, a_{l}} \sum_{a_{l-1}^{\prime}, a_{l}^{\prime}} \sum_{b_{l-1}, b_{l}} L_{a_{l-1}^{\prime}, b_{l-1}}^{a_{l-1}}\left(\sum_{\sigma_{l}, \sigma_{l}^{\prime}} M_{a_{l-1}, a_{l}}^{\sigma_{l} *} W_{b_{l-1}, b_{l}}^{\sigma_{l}, \sigma_{l}^{\prime}} M_{a_{l-1}^{\prime}, a_{l}^{\prime}}^{\sigma_{l}^{\prime *}}\right) R_{a_{l}^{\prime}, b_{l}}^{a_{l}}
\end{aligned}
$$

Whereby, $L_{a_{l-1}^{\prime}, b_{l-1}}^{a_{l-1}}$ represents the left side of the matrix at site 1 and $R_{a_{l}^{\prime}, b_{l}}^{a_{l}}$ the right side. They are explicitly given by:

$$
\begin{aligned}
L_{a_{l-1}^{\prime}, b_{l-1}}^{a_{l-1}} & =\sum_{\left\{a_{i}, a_{i}^{\prime}, b_{i} ; i<l-1\right\}}\left(\sum_{\sigma_{1}, \sigma_{1}^{\prime}} A_{1, a_{1}}^{\sigma_{1} *} W_{1, b_{1}}^{\sigma_{1}, \sigma_{1}^{\prime}} A_{1, a_{1}^{\prime}}^{\sigma_{1}^{\prime}}\right) \cdots\left(\sum_{\sigma_{l-1}, \sigma_{l-1}^{\prime}} A_{a_{l-2}, a_{l-1}}^{\sigma_{l-1} *} W_{b_{l-2}, b_{l-1}}^{\sigma_{l-1}, \sigma_{l-1}^{\prime}} A_{a_{l-2}^{\prime}, a_{l-1}^{\prime}}^{\sigma_{l-1}^{\prime}}\right) \\
R_{a_{l}^{\prime}, b_{l}}^{a_{l}} & =\sum_{\left\{a_{i}, a_{i}^{\prime}, b_{i} ; i>l\right\}}\left(\sum_{\sigma_{l+1}, \sigma_{l+1}^{\prime}} B_{a_{l}, a_{l+1}}^{\sigma_{l+1} *} W_{b_{l}, b_{l+1}}^{\sigma_{l+1}, \sigma_{l+1}^{\prime}} B_{a_{l}^{\prime}, a_{l+1}^{\prime}}^{\sigma_{l+1}^{\prime}}\right) \cdots\left(\sum_{\sigma_{L}, \sigma_{L}^{\prime}} A_{a_{L-1}, 1}^{\sigma_{L} *} W_{b_{L-1}, 1}^{\sigma_{L}, \sigma_{L}^{\prime}} A_{a_{L-1}^{\prime}, 1}^{\sigma_{L}^{\prime}}\right)
\end{aligned}
$$

Also the scalar product $\langle\psi \mid \psi\rangle$ can be given in local form:

$$
\begin{aligned}
\langle\psi \mid \psi\rangle & =\sum_{\sigma, \sigma^{\prime}}\left\langle\boldsymbol{\sigma}|B^{\sigma_{L} *} \cdots B^{\sigma_{l+1} *} M^{\sigma_{l} *} \underbrace{A^{\sigma_{l-1} *} \cdots A^{\sigma_{1} *} A^{\sigma_{1}^{\prime}} \cdots A^{\sigma_{l-1}^{\prime}}}_{1} M^{\sigma_{l}^{\prime}} B^{\sigma_{l+1}^{\prime}} \cdots B^{\sigma_{L}^{\prime}}| \boldsymbol{\sigma}^{\prime}\right\rangle \\
& =\sum_{\sigma_{l+1}, \ldots, \sigma_{L}} \sum_{a_{l}}(M^{\sigma_{l} *} M^{\sigma_{l}} \underbrace{B^{\sigma_{l+1}^{\prime}} \cdots B^{\sigma_{L}^{\prime}} B^{\sigma_{L} *} \cdots B^{\sigma_{l+1} *}}_{1})_{a_{l}, a_{l}} \\
& =\sum_{\sigma_{l}} \operatorname{Tr}\left(M^{\sigma_{l} *} M^{\sigma_{l}}\right)
\end{aligned}
$$

\section{Effective calculation of $L$ and $R$}

After the optimization of a single matrix at site $l$ one continues with the next site. In order to setup the new $L$ and $R$ tensors, one can use that the new $\mathrm{L}$ and $\mathrm{R}$ can be updated from the previous by: 


$$
\begin{aligned}
L_{a_{l-1}^{\prime}, b_{l-1}}^{a_{l-1}}= & \sum_{a_{l-2}, a_{l-2}^{\prime}, b_{l-2}} L_{a_{l-2}^{\prime}, b_{l-2}}^{a_{l-2}}\left(\sum_{\sigma_{l-1}, \sigma_{l-1}^{\prime}} A_{a_{l-2}, a_{l-1}}^{\sigma_{l-1} *} W_{b_{l-2}, b_{l-1}}^{\sigma_{l-1}, \sigma_{l-1}^{\prime}} A_{a_{l-2}^{\prime}, a_{l-1}^{\prime}}^{\sigma_{l-1}^{\prime}}\right) \\
= & \sum_{\sigma_{l-1}, \sigma_{l-1}^{\prime}} \sum_{a_{l-2}, a_{l-2}^{\prime}} A_{a_{l-2}, a_{l-1}}^{\sigma_{l-1} *}\left(\sum_{b_{l-2}} L_{a_{l-2}^{\prime}, b_{l-2}}^{a_{l-2}} W_{b_{l-2}, b_{l-1}}^{\sigma_{l-1}, \sigma_{l-1}^{\prime}}\right) A_{a_{l-2}^{\prime}, a_{l-1}^{\prime}}^{\sigma_{l-1}^{\prime}} \\
= & \sum_{\sigma_{l-1}, \sigma_{l-1}^{\prime}} \sum_{a_{l-2}, a_{l-2}^{\prime}} A_{a_{l-2}, a_{l-1}}^{\sigma_{l-1}}\left(L^{a_{l-2}} W^{\sigma_{l-1}, \sigma_{l-1}^{\prime}}\right)_{a_{l-2}^{\prime}, b_{l-1}} A_{a_{l-2}^{\prime}, a_{l-1}^{\prime}}^{\sigma_{l-1}^{\prime}} \\
= & \sum_{\sigma_{l-1}, \sigma_{l-1}^{\prime}} \sum_{a_{l-2}} A_{a_{l-2}, a_{l-1}}^{\sigma_{l-1}}\left(\sum_{a_{l-2}^{\prime}}\left(\left(L^{a_{l-2}} W^{\sigma_{l-1}, \sigma_{l-1}^{\prime}}\right)^{t}\right)_{b_{l-1}, a_{l-2}^{\prime}} A_{a_{l-2}^{\prime}, a_{l-1}^{\prime}}^{\sigma_{l-1}^{\prime}}\right) \\
= & \sum_{\sigma_{l-1}, \sigma_{l-1}^{\prime}} \sum_{a_{l-2}} A_{a_{l-2}, a_{l-1}}^{\sigma_{l-1}}\left(\left(L^{a_{l-2}} W^{\sigma_{l-1}, \sigma_{l-1}^{\prime}}\right)^{t} A^{\sigma_{l-1}^{\prime}}\right)_{b_{l-1}, a_{l-1}^{\prime}}
\end{aligned}
$$

Please note that $L^{a_{0}}$ is a scalar with value 1 . There is an analog formulation for $R_{a_{l}^{\prime}, b_{l}}^{a_{l}}$ :

$$
R_{a_{l}^{\prime}, b_{l}}^{a_{l}}=\sum_{\sigma_{l+1}, \sigma_{l+1}^{\prime}} \sum_{a_{l+1}} B_{a_{l}, a_{l+1}}^{\sigma_{l+1} *}\left(W^{\sigma_{l+1}, \sigma_{l+1}^{\prime}}\left(B^{\sigma_{l+1}^{\prime}} R^{a_{l+1}}\right)^{t}\right)_{b_{l}, a_{l}^{\prime}}
$$

\section{Sweeping Details}

At first the matrices of the starting state are filled with random values. Then the state is right-normalized and all $R$-matrices are constructed. The sweep will start at site $l=1$ and the MPS has the form :

$$
M^{\sigma_{1}} B^{\sigma_{2}} \cdots B^{\sigma_{L}}
$$

Next the $H_{\mathrm{Eff}}$ at site $l$ is constructed and the related local eigenvalue problem is solved. Then the matrix $M^{\sigma_{l}}$ is updated by the (reshaped) eigenvector of the smallest eigenvalue. In order to extend the left-normalized block a SVD on the new matrix $\tilde{M}^{\sigma_{l}}$ is applied and the $U$-matrix is saved as the new $A^{\sigma_{l}}$-matrix while the $S V^{\dagger}$-matrix is multiplied to the matrix $B^{\sigma_{l+1}}$ which shrinks the right block (2) :

$$
\begin{aligned}
A^{\sigma_{1}} \cdots A^{\sigma_{l-1}} M^{\sigma_{l}} B^{\sigma_{l+1}} \cdots B^{\sigma_{L}} & \stackrel{(1)}{\longrightarrow} A^{\sigma_{1}} \cdots A^{\sigma_{l-1}} \tilde{M}^{\sigma_{l}} B^{\sigma_{l+1}} \cdots B^{\sigma_{L}} \\
& \stackrel{(2)}{\longrightarrow} A^{\sigma_{1}} \cdots A^{\sigma_{l}} M^{\sigma_{l+1}} B^{\sigma_{l+2}} \cdots B^{\sigma_{L}}
\end{aligned}
$$

Then it is proceeded with the next site $(l \rightarrow l+1)$. Repeating this step until $l=L$ is reached, will give one complete right sweep. The MPS is now left-normalized:

$$
A^{\sigma_{1}} \cdots A^{\sigma_{L-1}} M^{\sigma_{L}}
$$

Analogously to the right step one can sweep also to the left side. The sweeping is stopped when a certain criteria is fullfilled (e.g. convergence, max. number of sweeps). 
We want to express the reorthogonalization equation

$$
\left|\tilde{\psi}_{n}\right\rangle=\left|f_{n}\right\rangle-\sum_{i}^{n-1}\left\langle f_{n} \mid \psi_{i}\right\rangle\left|\psi_{i}\right\rangle, \quad\left|\psi_{n}\right\rangle=\frac{1}{N_{n}}\left|\tilde{\psi}_{n}\right\rangle, \quad N_{n}=\sqrt{\left\langle\tilde{\psi}_{n} \mid \tilde{\psi}_{n}\right\rangle}
$$

in the form

$$
\left|\psi_{n}\right\rangle=\sum_{i}^{n} S_{i n}\left|f_{i}\right\rangle
$$

where we have introduced the reorthogonalization matrix $S_{i n}$ :

$$
\begin{aligned}
\left|\tilde{\psi}_{n}\right\rangle & =\left|f_{n}\right\rangle-\sum_{i}^{n-1}\left\langle f_{n} \mid \psi_{i}\right\rangle\left|\psi_{i}\right\rangle \\
& =\left|f_{n}\right\rangle-\sum_{i}^{n-1}\left\langle f_{n}\right|\left(\sum_{k}^{i} S_{k i}\left|f_{k}\right\rangle\right)\left(\sum_{k^{\prime}}^{i} S_{k^{\prime} i}\left|f_{k^{\prime}}\right\rangle\right) \\
& =\left|f_{n}\right\rangle-\sum_{i}^{n-1}\left(\sum_{k}^{i} S_{k i} W_{n k}\right)\left(\sum_{k^{\prime}}^{i} S_{k^{\prime} i}\left|f_{k^{\prime}}\right\rangle\right) \\
& =\left|f_{n}\right\rangle-\sum_{i}^{n-1} \sum_{k^{\prime}}^{i} k_{i n} S_{k^{\prime} i}\left|f_{k^{\prime}}\right\rangle, \\
k_{i n} & =\sum_{k}^{i} S_{k i} W_{n k}, \quad W_{i j}=\left\langle f_{i} \mid f_{j}\right\rangle .
\end{aligned}
$$

Now one can deduce a recursion formula for the matrix elements of $S$ :

$$
\tilde{S}_{p n}= \begin{cases}-\sum_{q=p}^{n-1} k_{q n} S_{p q}, & \text { if } p<n \\ 1, & \text { if } p=n\end{cases}
$$

with

$$
\begin{aligned}
S_{p n} & =\frac{1}{N_{n}} \tilde{S}_{p n}, \quad S_{00}=\frac{1}{N_{0}} \\
N_{n} & =\sqrt{\sum_{q, p=0}^{n} \tilde{S}_{p n} \tilde{S}_{q n} W_{p q}, \quad N_{0}=\sqrt{W_{00}}} .
\end{aligned}
$$

$W_{i j}$ is called the Gramian matrix. It gives a measure for the linear dependence of the Lanczos vectors. As long as det $W_{i j}>0$ one still adds linear independent vectors to the subspace. The matrix elements of the effective Hamiltonian are then given by:

$$
\left\langle\psi_{n}|H| \psi_{m}\right\rangle=\sum_{i, j}^{n, m} S_{i n}^{*} S_{j m}\left\langle f_{i}|H| f_{j}\right\rangle
$$


By construction the vectors $\left|\psi_{i}\right\rangle$ define an orthonormal basis system, i.e., on the left hand side we have ensured that the effective Hamiltonian is connected by a unitary transformation to the right hand side. In particular, one does not have to change the calculated Lanczos vectors any more. Since the vectors $\left|\psi_{i}\right\rangle$ are orthonormal, the spectral weight $\Omega_{k}$ belonging to the excitation energy $\epsilon_{k}$ can now be calculated very easily from ${ }^{1}$

$$
\Omega_{k} /\left\langle 0\left|\hat{A}^{\dagger} \hat{A}\right| 0\right\rangle=\left|\left\langle E_{k}|A| 0\right\rangle\right|^{2}=\left|\sum_{i} c_{k i}\left\langle\psi_{i} \mid \psi_{0}\right\rangle\right|^{2}=\left|c_{k 0}\right|^{2}
$$

where the exact diagonalization of the effective Hamiltonian yields the (approximate) eigenvectors $\left|E_{k}\right\rangle=\sum_{i} c_{k i}\left|\psi_{i}\right\rangle$. One can again express this residual without knowing the eigenvectors explicitly, for the additional price that one has to measure all the matrix elements for $H^{2}$. Then the quantities entering the residual can be calculated as

$$
\begin{aligned}
\left\langle E_{k}|H| E_{k}\right\rangle & =\sum_{i j} c_{k i} c_{k j} \sum_{p, q}^{i, j} S_{p i} S_{q j}\left\langle f_{p}|H| f_{q}\right\rangle, \\
\left\langle E_{k}\left|H^{2}\right| E_{k}\right\rangle & =\sum_{i j} c_{k i} c_{k j}\left\langle\psi_{i}\left|H^{2}\right| \psi_{j}\right\rangle \\
& =\sum_{i j} c_{k i} c_{k j} \sum_{p, q}^{i, j} S_{p i} S_{q j}\left\langle f_{p}\left|H^{2}\right| f_{q}\right\rangle .
\end{aligned}
$$

\footnotetext{
${ }^{1}$ This approach is thus, besides being numerically more stable, also much simpler as compared to the adaptive Lanczos method, $\left[\mathrm{DHP}^{+}{ }^{11}\right]$ where one had to evaluate an integral in the complex plane.
} 


\section{Long range Kondo signature}

\section{Free Green's function with surface

The LCAO calculations have to include the decay of the wavefunctions at the surface. This decay depends on the energy and the wave vector component parallel to the surface:

$$
\kappa_{\mathbf{k}, \nu}=\sqrt{\frac{2 m}{\hbar^{2}}\left(\Phi-E_{\mathbf{k}, \nu}\right)+\mathbf{k}_{\|}^{2}}
$$

Here $\Phi=4.65 \mathrm{eV}$ is the work function and the energy $E$ is measured with respect to the Fermi-Level. The surface is orientated perpendicular to the z-axis and defines the border between the two adjacent subspaces crystal $(z \leq 0)$ and vacuum $(z>0)$. As the fcc band structure is invariant under reflections at the (001)-plane, the wave functions are:

$$
\begin{array}{cc}
\Psi_{\mathbf{k}, \nu}\left(\mathbf{x}_{\|}, z\right)=\left(1+\left|r_{\mathbf{k}, \nu}\right|^{2}\right)^{-1 / 2} e^{i \mathbf{k}_{\|} \mathbf{x}_{\|}\left[e^{i k_{z} z}+r_{\mathbf{k}, \nu} e^{-i k_{z} z}\right]} & z \leq 0 \\
\Psi_{\mathbf{k}, \nu}\left(\mathbf{x}_{\|}, z\right)=\left(1+\left|r_{\mathbf{k}, \nu}\right|^{2}\right)^{-1 / 2} e^{i \mathbf{k}_{\|} \mathbf{x}_{\|}}\left(1+r_{\mathbf{k}, \nu}\right) e^{-\kappa_{\mathbf{k}, \nu}} & z>0
\end{array}
$$

with the reflection coefficient $r_{\mathbf{k}, \nu}=\left(i k_{z}+\kappa_{\mathbf{k}, \nu}\right) /\left(i k_{z}-\kappa_{\mathbf{k}, \nu}\right)$ providing continuity of the wave-functions and their spatial derivative at the surface. The LDOS of the unperturbed system in both subspaces is then:

$$
\varrho_{0}(z, \omega)=\sum_{\nu} \int_{1 . B Z} d^{3} \mathbf{k} \delta\left(\omega-\epsilon_{\mathbf{k}, \nu}\right)\left|\Psi_{\mathbf{k}, \nu}\left(\mathbf{x}_{\|}, z\right)\right|^{2}
$$

The STM-Tip always probes the LDOS in the vacuum and the impurity is always located within the crystal. Therefore only the Green's function connecting a position $\mathbf{x}^{\prime}=(0,0,-d)$ inside the crystal with a position $\mathbf{x}=(x, y, h)$ in the vacuum is of interest. Here $d$ is the depths of the impurity below the surface and $h$ is the tip to sample distance. This particular Green's function is given by:

$$
G^{0}\left(\mathbf{x}_{\|}, h, d, \omega\right)=\sum_{\nu} \int_{1 . B Z} d^{3} \mathbf{k} \frac{\left(1+r_{\mathbf{k}, \nu}\right) e^{i\left(\mathbf{k}_{\|} \mathbf{x}_{\|}+k_{\perp} d\right)-\kappa_{\mathbf{k}, \nu} h}}{\left(1+\left|r_{\mathbf{k}, \nu}\right|^{2}\right)\left(\omega-E_{\mathbf{k}, \nu}+i 0^{+}\right)}
$$

The propagator for the opposite direction is identical due to time reversal symmetry. 


\section{\begin{tabular}{l|l} 
Fano line shapes & B.2
\end{tabular}}

Fano line shapes can be calculated for the LDOS, for the explicit derivation see also Ref. [Dar09]:

$$
\begin{aligned}
\Delta \operatorname{LDOS}(R, \omega) & =\frac{\left(\rho_{0 R}^{0}(\omega)\right)^{2}}{\rho^{0}(\omega)} \frac{q^{2}(\omega)-1+2 q(\omega) \Omega(\omega)}{\left.\Omega^{2}(\omega)+1\right)} \\
\text { with } \quad q(\omega) & =\frac{\operatorname{Re}\left(G_{0 R}^{0}\right)(\omega)}{\operatorname{Im}\left(G_{0 R}^{0}\right)(\omega)}, \quad \Omega(\omega)=\frac{\omega-\tilde{\epsilon_{d}}}{\Delta(\omega)}
\end{aligned}
$$

To write the spectral function as a Fano function Ujjághy neglected the energy dependence of $q(\omega)$ and $\Delta(\omega)$ and in the limit $R=0$ the LDOS can be described with the typical Fano functions:

$$
\operatorname{LDOS}(R=0, \omega)=\rho_{0}(\omega)=\rho_{0}^{0}+\delta \rho_{0}=\rho^{0} \frac{(\Omega+q)^{2}}{\left(\Omega^{2}+1\right)}
$$

Fano line shapes appear in general if a single level (state) is coupled to a continuum of states. Therefore simple potential scattering modelled by a Lorentzian resonance will also lead to Fano line shapes. However there is no need for the approximation done by Újsághy. 
List of references 



\section{in alphabetical order of labels}

[Abr65] Abrikosov A. Physics 2, 5 (1965).

[ABS08] Affleck I., Borda L. and Saleur H., Friedel oscillations and the Kondo screening cloud. Phys. Rev. B, 77(18), 180404 (2008), URL http://dx.doi.org/10.1103/PhysRevB.77. 180404.

[AFC09] Alba V., Fagotti M. and Calabrese P., Entanglement entropy of excited states. J. Stat. Mech.: Th. and Exp., 2009(10), P10020 (2009), URL http://dx.doi.org/10.1088/ $1742-5468 / 2009 / 10 /$ P 10020 .

[Aff09] Affleck I., The Kondo screening cloud: what it is and how to observe it. Review article for "Perspectives of Mesoscopic Physics - Dedicated to Prof Yoseph Imry's 70th Birthday" (2009), URL http://dx.doi.org/arXiv:0911.2209.

[AGO75] Andres K., Graebner J.E. and Ott H.R., 4f-Virtual-Bound-State Formation in $\mathrm{CeAl}_{3}$ at Low Temperatures. Phys. Rev. Lett., 35, 1779 (1975), URL http://dx.doi.org/10.1103/ PhysRevLett.35.1779.

[AKLT87] Affleck I., Kennedy T., Lieb E.H. and Tasaki H., Rigorous results on valence-bond ground states in antiferromagnets. Phys. Rev. Lett., 59, 799 (1987), URL http://dx.doi.org/ 10.1103/PhysRevLett.59.799.

[AM76] Ashcroft N.W. and Mermin N.D., Solid State Physics, (Thomson Learning, 1976), 1st edition.

[And61] Anderson P.W., Localized Magnetic States in Metals. Phys. Rev., 124(1), 41 (1961), URL http://dx.doi.org/10.1103/PhysRev.124.41.

[And66] Anderson P.W., Localized Magnetic States and Fermi-Surface Anomalies in Tunneling. Phys. Rev. Lett., 17(2), 95 (1966), URL http://dx.doi.org/10.1103/PhysRevLett.17. 95.

[And70] Anderson P.W., A poor man's derivation of scaling laws for the Kondo problem. J. Phys. C: Sol. Stat. Phys., 3(12), 2436 (1970), URL http://dx.doi.org/10.1088/0022-3719/ 3/12/008.

[And72] Anderson P.W., More Is Different. Science, 177(4047), 393 (1972), URL http://dx.doi. org/10.1126/science.177.4047.393.

[And73] Anderson P., Resonating valence bonds: A new kind of insulator? Materials Research Bulletin, 8(2), 153 (1973), URL http://dx.doi.org/10.1016/0025-5408(73)90167-0.

[And80] Andrei N., Diagonalization of the Kondo Hamiltonian. Phys. Rev. Lett., 45(5), 379 (1980), URL http://dx.doi.org/10.1103/PhysRevLett.45.379.

[AS01] Affleck I. and Simon P., Detecting the Kondo Screening Cloud Around a Quantum Dot. Phys. Rev. Lett., 86(13), 2854 (2001), URL http://dx.doi.org/10.1103/PhysRevLett. 86.2854.

[AS05] Anders F.B. and Schiller A., Real-Time Dynamics in Quantum-Impurity Systems: A TimeDependent Numerical Renormalization-Group Approach. Phys. Rev. Lett., 95, 196801 (2005), URL http://dx.doi.org/10.1103/PhysRevLett.95.196801.

[AS06] Anders F.B. and Schiller A., Spin precession and real-time dynamics in the Kondo model:Time-dependent numerical renormalization-group study. Phys. Rev. B, 74, 245113 (2006), URL http://dx.doi.org/10.1103/PhysRevB.74.245113. 
[BA96] Barzykin V. and Affleck I., The Kondo Screening Cloud: What Can We Learn from Perturbation Theory? Phys. Rev. Lett., 76(26), 4959 (1996), URL http://dx.doi.org/10. 1103/PhysRevLett. 76.4959.

[BA98] Barzykin V. and Affleck I., Screening cloud in the $k$-channel Kondo model: Perturbative and large-k results. Phys. Rev. B, 57, 432 (1998), URL http://dx.doi.org/10.1103/ PhysRevB . 57.432.

[Bal10] Balents L., Spin liquids in frustrated magnets. Nature, 464(7286), 199 (2010), URL http://dx.doi.org/10.1038/nature08917.

[Bax68] Baxter R.J., Dimers on a Rectangular Lattice. Journal of Mathematical Physics, 9(4), 650 (1968), URL http://dx.doi.org/10.1063/1.1664623.

[BCP08] Bulla R., Costi T.A. and Pruschke T., Numerical renormalization group method for quantum impurity systems. Rev. Mod. Phys., 80, 395 (2008), URL http://dx.doi.org/10. 1103/RevModPhys.80.395.

[BCS57a] Bardeen J., Cooper L.N. and Schrieffer J.R., Microscopic Theory of Superconductivity. Phys. Rev., 106, 162 (1957), URL http://dx.doi.org/10.1103/PhysRev.106.162.

[BCS57b] Bardeen J., Cooper L.N. and Schrieffer J.R., Theory of Superconductivity. Phys. Rev., 108, 1175 (1957), URL http://dx.doi.org/10.1103/PhysRev.108.1175.

[BCV01] Bulla R., Costi T.A. and Vollhardt D., Finite-temperature numerical renormalization group study of the Mott transition. Phys. Rev. B, 64, 045103 (2001), URL http://dx.doi.org/ 10.1103/PhysRevB.64.045103.

[BD87] Box G. and Draper N., Empirical model-building and response surfaces., (John Wiley \& Sons, 1987).

$\left[\mathrm{BDD}^{+} 00\right] \quad$ Bai Z., Demmel J., Dongarra J., Ruhe A. and van der Vorst H., Templates for the Solution of Eigenvalue Problems: A Practical Guide, (SIAM, Philadelphia, 2000).

[Ber08] Bergmann G., Friedel oscillations near Kondo impurities: A comparison of numerical calculation methods. Phys. Rev. B, 78(19), 195124 (2008), URL http://dx.doi.org/10. 1103/PhysRevB .78.195124.

[Bet31] Bethe H., Zur Theorie der Metalle. Z. Physik, 71, 205 (1931), URL http://dx.doi.org/ 10.1007/BF01341708.

[Bet47] Bethe H.A., The Electromagnetic Shift of Energy Levels. Phys. Rev., 72, 339 (1947), URL http://dx.doi.org/10.1103/PhysRev.72.339.

[BGJ04] Benthien H., Gebhard F. and Jeckelmann E., Spectral Function of the One-Dimensional Hubbard Model away from Half Filling. Phys. Rev. Lett., 92(25), 256401 (2004), URL http://dx.doi.org/10.1103/PhysRevLett.92.256401.

[BGLP00] Bulla R., Glossop M.T., Logan D.E. and Pruschke T., The soft-gap Anderson model: comparison of renormalization group and local moment approaches. J. Phys.: Cond. Matt., 12, 4899 (2000), URL http://dx. doi.org/10.1088/0953-8984/12/23/302.

[BHP98] Bulla R., Hewson A.C. and Pruschke T., Numerical renormalization group calculations for the self-energy of the impurity Anderson model. J. Phys.: Cond. Matt., 10(37), 8365 (1998), URL http://dx.doi.org/10.1088/0953-8984/10/37/021.

[BHV06] Bravyi S., Hastings M.B. and Verstraete F., Lieb-Robinson Bounds and the Generation of Correlations and Topological Quantum Order. Phys. Rev. Lett., 97, 050401 (2006), URL http://dx.doi.org/10.1103/PhysRevLett.97.050401.

[BJ07] Benthien H. and Jeckelmann E., Spin and charge dynamics of the one-dimensional extended Hubbard model. Phys. Rev. B, 75(20), 205128 (2007), URL http://dx.doi .org/10.1103/ PhysRevB.75.205128.

[BKL $\left.{ }^{+} 11\right] \quad$ Bouillot P., Kollath C., Läuchli A.M., Zvonarev M., Thielemann B., Rüegg C., Orignac E., Citro R., Klanjsek M., Berthier C., Horvatic M. and Giamarchi T., Statics and dynamics 
of weakly coupled antiferromagnetic spin- $\frac{1}{2}$ ladders in a magnetic field. Phys. Rev. B, 83, 054407 (2011), URL http://dx.doi.org/10.1103/PhysRevB.83.054407.

[Blu01] Blundell S., Magnetism in condensed matter, volume 54, (Oxford University Press, 2001).

[BM86] Bednorz J. and Müller K., Possible high $T_{C}$ superconductivity in the Ba-La-Cu-O systems. Zeit. f. Phys. B Cond. Mat., 64(2), 189 (1986), URL http://dx.doi.org/10.1007/ BF01303701.

[Bor07] Borda L., Kondo screening cloud in a one-dimensional wire: Numerical renormalization group study. Phys. Rev. B, 75(4), 041307 (2007), URL http://dx.doi.org/10.1103/ PhysRevB. 75.041307.

[BR87] Binnig G. and Rohrer H., Scanning tunneling microscopy - from birth to adolescence. Rev. Mod. Phys., 59, 615 (1987), URL http://dx.doi.org/10.1103/RevModPhys.59.615.

[BR99] Binnig G. and Rohrer H., In touch with atoms. Rev. Mod. Phys., 71, S324 (1999), URL http://dx.doi.org/10.1103/RevModPhys.71.S324.

[Bru04] Bruus F., Many-Body Quantum Theory in Condensed Matter Physics, (Oxford Greaduate Texts, 2004), 1st edition.

[BSW09] Barthel T., Schollwöck U. and White S.R., Spectral functions in one-dimensional quantum systems at finite temperature using the density matrix renormalization group. Phys. Rev. B, 79, 245101 (2009), URL http://dx.doi.org/10.1103/PhysRevB.79.245101.

[Cal70] Callan C.G., Broken Scale Invariance in Scalar Field Theory. Phys. Rev. D, 2, 1541 (1970), URL http://dx.doi.org/10.1103/PhysRevD.2.1541.

$\left[\mathrm{CBW}^{+}\right.$09] Costi T.A., Bergqvist L., Weichselbaum A., von Delft J., Micklitz T., Rosch A., Mavropoulos P., Dederichs P.H., Mallet F., Saminadayar L. and Bäuerle C., Kondo Decoherence: Finding the Right Spin Model for Iron Impurities in Gold and Silver. Phys. Rev. Lett., 102, 056802 (2009), URL http://dx.doi.org/10.1103/PhysRevLett.102.056802.

[CEE08] Chubukov A.V., Efremov D.V. and Eremin I., Magnetism, superconductivity, and pairing symmetry in iron-based superconductors. Phys. Rev. B, 78, 134512 (2008), URL http:// dx.doi.org/10.1103/PhysRevB.78.134512.

[CGTR06] Costamagna S., Gazza C.J., Torio M.E. and Riera J.A., Anderson impurity in the onedimensional Hubbard model for finite-size systems. Phys. Rev. B, 74(19), 195103 (2006), URL http://dx.doi.org/10.1103/PhysRevB.74.195103.

[CH90] Costi T. and Hewson A., A new approach to the calculation of spectra for strongly correlated systems. Physica B: Cond. Mat., 163, 179 (1990), URL http://dx.doi.org/10.1016/ 0921-4526 (90) 90161-M.

[CH06] Caux J.S. and Hagemans R., The four-spinon dynamical structure factor of the Heisenberg chain. J. Stat. Mech.: Th. and Exp., 2006(12), P12013 (2006), URL http://dx.doi.org/ $10.1088 / 1742-5468 / 2006 / 12 /$ P12013.

[Col12] Coleman P., Introduction to Many Body Physics (, 2012), URL http://www.physics. rutgers . edu/ coleman/620/mbody/pdf/bkx.pdf.

[CW85] Cullum J. and Willoughby R., Lanczos algorithms for large symmetric eigenvalue computations, volume 2, (Birkhäuser, Boston, 1985).

[Dar09] Dargel P.E., Local Density of States of the Single Impurity Anderson Model, Thesis, GeorgAugust-Universität Göttingen (2009).

[DBTR12] Dolfi M., Bauer B., Troyer M. and Ristivojevic Z., Multigrid Algorithms for Tensor Network States. Phys. Rev. Lett., 109, 020604 (2012), URL http://dx.doi.org/10.1103/ PhysRevLett.109.020604.

[dCP62] des Cloizeaux J. and Pearson J.J., Spin-Wave Spectrum of the Antiferromagnetic Linear Chain. Phys. Rev., 128(5), 2131 (1962), URL http://dx.doi.org/10.1103/PhysRev. 128.2131. 
[DHP $\left.{ }^{+} 11\right] \quad$ Dargel P.E., Honecker A., Peters R., Noack R.M. and Pruschke T., Adaptive Lanczos-vector method for dynamic properties within the density matrix renormalization group. Phys. Rev. B, 83(16), 161104 (2011), URL http://dx.doi.org/10.1103/PhysRevB.83.161104.

[DMDNS98] Dukelsky J., Martín-Delgado M.A., Nishino T. and Sierra G., Equivalence of the variational matrix product method and the density matrix renormalization group applied to spin chains. EPL, 43(4), 457 (1998), URL http://dx.doi.org/10.1209/epl/i1998-00381-x.

[DS68] Daybell M.D. and Steyert W.A., Localized Magnetic Impurity States In Metals: Some Experimental Relationships. Rev. Mod. Phys., 40, 380 (1968), URL http://dx.doi.org/ 10.1103/RevModPhys . 40.380.

$\left[\mathrm{DWH}^{+} 12\right]$ Dargel P.E., Wöllert A., Honecker A., McCulloch I.P., Schollwöck U. and Pruschke T., Lanczos algorithm with matrix product states for dynamical correlation functions. Phys. Rev. B, 85, 205119 (2012), URL http://dx.doi.org/10.1103/PhysRevB.85.205119.

[Dys49a] Dyson F.J., The Radiation Theories of Tomonaga, Schwinger, and Feynman. Phys. Rev., 75, 486 (1949), URL http://dx.doi.org/10.1103/PhysRev.75.486.

[Dys49b] Dyson F.J., The S Matrix in Quantum Electrodynamics. Phys. Rev., 75, 1736 (1949), URL http://dx.doi.org/10.1103/PhysRev.75.1736.

[ECP10] Eisert J., Cramer M. and Plenio M.B., Colloquium: Area laws for the entanglement entropy. Rev. Mod. Phys., 82, 277 (2010), URL http://dx.doi.org/10.1103/RevModPhys. 82.277 .

[EHR $\left.{ }^{+} 07\right] \quad$ Ehm D., Hüfner S., Reinert F., Kroha J., Wölfle P., Stockert O., Geibel C. and Löhneysen H.v., High-resolution photoemission study on low- $T_{K}$ Ce systems: Kondo resonance, crystal field structures, and their temperature dependence. Phys. Rev. B, 76, 045117 (2007), URL http://dx.doi.org/10.1103/PhysRevB.76.045117.

[Eig73] Eigen M., The Physicists's Conception of Nature, volume 618, (Springer, 1973).

[Eis06] Eisert J., Computational Difficulty of Global Variations in the Density Matrix Renormalization Group. Phys. Rev. Lett., 97, 260501 (2006), URL http://dx.doi.org/10.1103/ PhysRevLett.97.260501.

[EO06] Eisert J. and Osborne T.J., General Entanglement Scaling Laws from Time Evolution. Phys. Rev. Lett., 97, 150404 (2006), URL http://dx.doi.org/10.1103/PhysRevLett. 97.150404.

[EV11] Evenbly G. and Vidal G., Tensor Network States and Geometry. Journal of Statistical Physics, 145, 891 (2011), URL http://dx.doi.org/10.1007/s10955-011-0237-4.

[Fan61] Fano U., Effects of Configuration Interaction on Intensities and Phase Shifts. Phys. Rev., 124(6), 1866 (1961), URL http://dx.doi.org/10.1103/PhysRev.124.1866.

[FCC09] Faribault A., Calabrese P. and Caux J.S., Bethe ansatz approach to quench dynamics in the Richardson model. J. Math. Phys, 50(9), 095212 (2009), URL http://dx.doi.org/ DOI : $10.1063 / 1.3183720$.

$\left[\mathrm{FCM}^{+}\right.$08] Flesch A., Cramer M., McCulloch I.P., Schollwöck U. and Eisert J., Probing local relaxation of cold atoms in optical superlattices. Phys. Rev. A, 78, 033608 (2008), URL http://dx. doi.org/10.1103/PhysRevA.78.033608.

[Fey49a] Feynman R.P., Space-Time Approach to Quantum Electrodynamics. Phys. Rev., 76, 769 (1949), URL http://dx.doi.org/10.1103/PhysRev.76.769.

[Fey49b] Feynman R.P., The Theory of Positrons. Phys. Rev., 76, 749 (1949), URL http://dx. doi.org/10.1103/PhysRev.76.749.

[Fey50] Feynman R.P., Mathematical Formulation of the Quantum Theory of Electromagnetic Interaction. Phys. Rev., 80, 440 (1950), URL http://dx.doi.org/10.1103/PhysRev.80. 440. 
[FKMS07] Friedrich A., Kolezhuk A.K., McCulloch I.P. and Schollwöck U., Edge singularities in highenergy spectra of gapped one-dimensional magnets in strong magnetic fields. Phys. Rev. B, 75, 094414 (2007), URL http://dx.doi.org/10.1103/PhysRevB.75.094414.

[FKMW95] Fledderjohann A., Karbach M., Mutter K.H. and Wielath P., Computation of dynamical structure factors with the recursion method. J. Phys.: Cond. Matt., 7(47), 8993 (1995), URL http://dx.doi.org/10.1088/0953-8984/7/47/018.

[FND10] Fröwis F., Nebendahl V. and Dür W., Tensor operators: Constructions and applications for long-range interaction systems. Phys. Rev. A, 81, 062337 (2010), URL http://dx. doi.org/10.1103/PhysRevA.81.062337.

[FNW92] Fannes M., Nachtergaele B. and Werner R., Finitely correlated states on quantum spin chains. Comm. in Math. Phys., 144, 443 (1992), URL http://dx.doi.org/10.1007/ BF02099178.

[FO86] Frota H.O. and Oliveira L.N., Photoemission spectroscopy for the spin-degenerate Anderson model. Phys. Rev. B, 33(11), 7871 (1986), URL http://dx.doi.org/10.1103/PhysRevB. 33. 7871.

[Fro92] Frota H.O., Shape of the Kondo resonance. Phys. Rev. B, 45, 1096 (1992), URL http:// dx.doi.org/10.1103/PhysRevB.45.1096.

[FT81] Faddeev L.D. and Takhtajan L.A., What is the spin of a spin wave? Physics Letters A, 85(6-7), 375 (1981), URL http://dx.doi.org/DOI : 10.1016/0375-9601 (81)90335-2.

[GB87] Gagliano E.R. and Balseiro C.A., Dynamical Properties of Quantum Many-Body Systems at Zero Temperature. Phys. Rev. Lett., 59(26), 2999 (1987), URL http://dx.doi.org/ 10.1103/PhysRevLett.59.2999.

[GGSM ${ }^{+}$98] Goldhaber-Gordon D., Shtrikman H., Mahalu D., Abusch-Magder D., Meirav U. and Kastner M., Kondo effect in a single-electron transistor. Nature, 391(6663), 156 (1998), URL http://dx.doi.org/10.1038/34373.

[GHR04] Garcia D.J., Hallberg K. and Rozenberg M.J., Dynamical Mean Field Theory with the Density Matrix Renormalization Group. Phys. Rev. Lett., 93, 246403 (2004), URL http:// dx.doi.org/10.1103/PhysRevLett.93.246403.

[GHS87] Gubernatis J.E., Hirsch J.E. and Scalapino D.J., Spin and charge correlations around an Anderson magnetic impurity. Phys. Rev. B, 35(16), 8478 (1987), URL http://dx.doi. org/10.1103/PhysRevB.35.8478.

[GL50] Ginzburg V. and Landau L., On the theory of superconductivity. Zh. Eksp. Teor. Fiz., 20, 1064 (1950).

[GML54] Gell-Mann M. and Low F.E., Quantum Electrodynamics at Small Distances. Phys. Rev., 95, 1300 (1954), URL http://dx.doi.org/10.1103/PhysRev.95.1300.

[Gol92] Goldenfeld N., Lectures on phase transitions and the renormalization group, (AddisonWesley, Advanced Book Program, Reading, 1992).

[GR06] Garcia-Ripoll J.J., Time evolution of Matrix Product States. New Journal of Physics, 8(12), 305 (2006), URL http://dx.doi.org/10.1088/1367-2630/8/12/305.

[GS83a] Gunnarsson O. and Schönhammer K., Electron spectroscopies for Ce compounds in the impurity model. Phys. Rev. B, 28, 4315 (1983), URL http://dx.doi.org/10.1103/ PhysRevB.28.4315.

[GS83b] Gunnarsson O. and Schönhammer K., Photoemission from Ce Compounds: Exact Model Calculation in the Limit of Large Degeneracy. Phys. Rev. Lett., 50, 604 (1983), URL http://dx.doi.org/10.1103/PhysRevLett.50.604.

[GS85] Gunnarsson O. and Schönhammer K., Double occupancy of the f orbital in the Anderson model for Ce compounds. Phys. Rev. B, 31, 4815 (1985), URL http://dx.doi.org/10. 1103/PhysRevB. 31.4815. 
[GSR $\left.{ }^{+} 11\right] \quad$ Grete P., Schmitt S., Raas C., Anders F.B. and Uhrig G.S., Kinks in the electronic dispersion of the Hubbard model away from half filling. Phys. Rev. B, 84, 205104 (2011), URL http://dx.doi.org/10.1103/PhysRevB.84.205104.

[GvL96] Golub G. and van Loan C., Matrix Computations, (The John Hopkins University Press, Baltimore, 1996).

[GWS $\left.{ }^{+} 04\right] \quad$ Garleff J.K., Wenderoth M., Sauthoff K., Ulbrich R.G. and Rohlfing M., $2 \times 1$ reconstructed Si(111) surface: STM experiments versus ab initio calculations. Phys. Rev. B, 70, 245424 (2004), URL http://dx.doi.org/10.1103/PhysRevB.70.245424.

[Hae11] Haegeman J., Variational Renormalization Group Methods for Extended Quantum Systems, $\mathrm{PhD}$ Thesis, Faculty of Sciences, Department of Physics and Astronomy, University Gent (2011).

[Hal95] Hallberg K.A., Density-matrix algorithm for the calculation of dynamical properties of lowdimensional systems. Phys. Rev. B, 52(14), R9827 (1995), URL http://dx.doi.org/10. 1103/PhysRevB.52.R9827.

[Has07a] Hastings M.B., An area law for one-dimensional quantum systems. J. Stat. Mech.: Th. and Exp., 2007(08), P08024 (2007), URL http://dx.doi.org/10.1088/1742-5468/2007/ 08/P08024.

[Has07b] Hastings M.B., Entropy and entanglement in quantum ground states. Phys. Rev. B, 76, 035114 (2007), URL http://dx.doi.org/10.1103/PhysRevB.76.035114.

[Hay80] Haydock R., The recursive solution of the Schrödinger equation. Comp. Phys. Comm., 20(1), 11 (1980), URL http://dx.doi.org/10.1016/0010-4655(80)90101-0.

$\left[\mathrm{HCO}^{+} 11\right] \quad$ Haegeman J., Cirac J.I., Osborne T.J., Pižorn I., Verschelde H. and Verstraete F., TimeDependent Variational Principle for Quantum Lattices. Phys. Rev. Lett., 107, 070601 (2011), URL http://dx.doi.org/10.1103/PhysRevLett.107.070601.

[Hei28] Heisenberg W., Zur Theorie des Ferromagnetismus. Zeitschrift für Physik A: Hadrons and Nuclei, 49, 619 (1928), URL http://dx.doi.org/10.1007/BF01328601.

[Hew93] Hewson A.C., The Kondo Problem to Heavy Fermions, (Cambridge University Press, 1993).

[HHK72] Haydock R., Heine V. and Kelly M.J., Electronic structure based on the local atomic environment for tight-binding bands. J. Phys. C: Sol. Stat. Phys., 5(20), 2845 (1972), URL http://dx.doi.org/10.1088/0022-3719/5/20/004.

[Hil37] Hildebrand E., Zum Dia- und Paramagnetismus in metallischen Mischkristallreihen. Das Verhalten von geloestem Kobalt und Rhodium. Ann. d. Physik, 30(5) (1937), URL http:// dx.doi.org/10.1002/andp. 19374220703.

[HKM06] Hand T., Kroha J. and Monien H., Spin Correlations and Finite-Size Effects in the OneDimensional Kondo Box. Phys. Rev. Lett., 97(13), 136604 (2006), URL http://dx.doi. org/10.1103/PhysRevLett.97.136604.

[HM07] Henzl J. and Morgenstern K., Contribution of the Surface State to the Observation of the Surface Kondo Resonance. Phys. Rev. Lett., 98, 266601 (2007), URL http://dx.doi. org/10.1103/PhysRevLett.98.266601.

[HMS ${ }^{+}$09] Holzner A., McCulloch I.P., Schollwöck U., von Delft J. and Heidrich-Meisner F., Kondo screening cloud in the single-impurity Anderson model: A density matrix renormalization group study. Phys. Rev. B, 80(20), 205114 (2009), URL http://dx.doi.org/10.1103/ PhysRevB.80.205114.

$\left[\mathrm{HPW}^{+} 12\right]$ Haegeman J., Pirvu B., Weir D.J., Cirac J.I., Osborne T.J., Verschelde H. and Verstraete F., Variational matrix product ansatz for dispersion relations. Phys. Rev. B, 85, 100408 (2012), URL http://dx.doi.org/10.1103/PhysRevB.85.100408.

[HSZ85] Horvatić B., Sokcević D. and Zlatić V., Density of states for intermediate valence and Kondo systems. Zeit. f. Phys. B Cond. Mat., 59, 151 (1985), URL http://dx.doi.org/ 10.1007/BF01725531. 
[HSZ87] Horvatić B., Sokcević D. and Zlatić V., Finite-temperature spectral density for the Anderson model. Phys. Rev. B, 36, 675 (1987), URL http://dx.doi.org/10.1103/PhysRevB.36. 675.

[Hüf03] Hüfner S., Photoelectron spectroscopy: principles and applications, (Springer Verlag, 2003).

$\left[\mathrm{HWM}^{+} 11\right]$ Holzner A., Weichselbaum A., McCulloch I.P., Schollwöck U. and von Delft J., Chebyshev matrix product state approach for spectral functions. Phys. Rev. B, 83(19), 195115 (2011), URL http://dx.doi.org/10.1103/PhysRevB.83.195115.

[HWvD10] Holzner A., Weichselbaum A. and von Delft J., Matrix product state approach for a twolead multilevel Anderson impurity model. Phys. Rev. B, 81, 125126 (2010), URL http:// dx.doi.org/10.1103/PhysRevB .81.125126.

[IDH06] Iancu V., Deshpande A. and Hla S.W., Manipulating Kondo Temperature via Single Molecule Switching. Nano Lett., 6(4), 820 (2006), URL http://dx.doi.org/10.1021/ n10601886.

[IFT98] Imada M., Fujimori A. and Tokura Y., Metal-insulator transitions. Rev. Mod. Phys., 70, 1039 (1998), URL http://dx.doi.org/10.1103/RevModPhys.70.1039.

[Jec02] Jeckelmann E., Dynamical density-matrix renormalization-group method. Phys. Rev. B, 66(4), 045114 (2002), URL http://dx.doi.org/10.1103/PhysRevB.66.045114.

[Jec03] Jeckelmann E., Optical excitations in a one-dimensional Mott insulator. Phys. Rev. B, 67, 075106 (2003), URL http://dx.doi.org/10.1103/PhysRevB.67.075106.

$\left[\mathrm{JFG}^{+}\right.$09] Jompol Y., Ford C., Griffiths J., Farrer I., Jones G., Anderson D., Ritchie D., Silk T. and Schofield A., Probing spin-charge separation in a Tomonaga-Luttinger liquid. Science, 325(5940), 597 (2009), URL http://dx.doi.org/10.1126/science.1171769.

[JG89] Jones R.O. and Gunnarsson O., The density functional formalism, its applications and prospects. Rev. Mod. Phys., 61, 689 (1989), URL http://dx.doi.org/10.1103/ RevModPhys.61.689.

[JKmW81] Jayaprakash C., Krishna-murthy H.R. and Wilkins J.W., Two-Impurity Kondo Problem. Phys. Rev. Lett., 47, 737 (1981), URL http://dx.doi.org/10.1103/PhysRevLett.47. 737.

[JOK $\left.{ }^{+} 11\right]$ Jeschke H., Opahle I., Kandpal H., ValentíR., Das H., Saha-Dasgupta T., Janson O., Rosner H., Brühl A., Wolf B., Lang M., Richter J., Hu S., Wang X., Peters R., Pruschke T. and Honecker A., Multistep Approach to Microscopic Models for Frustrated Quantum Magnets: The Case of the Natural Mineral Azurite. Phys. Rev. Lett., 106, 217201 (2011), URL http://dx.doi.org/10.1103/PhysRevLett.106.217201.

[JV87] Jones B.A. and Varma C.M., Study of two magnetic impurities in a Fermi gas. Phys. Rev. Lett., 58, 843 (1987), URL http://dx.doi.org/10.1103/PhysRevLett.58.843.

[JV89] Jones B.A. and Varma C.M., Critical point in the solution of the two magnetic impurity problem. Phys. Rev. B, 40, 324 (1989), URL http://dx.doi.org/10.1103/PhysRevB.40. 324.

[JVW88] Jones B.A., Varma C.M. and Wilkins J.W., Low-Temperature Properties of the TwoImpurity Kondo Hamiltonian. Phys. Rev. Lett., 61, 125 (1988), URL http://dx.doi. org/10.1103/PhysRevLett.61.125.

[Kad66] Kadanoff L.P., Scaling laws for Ising models near $T_{C}$. Physics, 2, 263 (1966).

[Kas56] Kasuya T., A Theory of Metallic Ferro- and Antiferromagnetism on Zener's Model. Progress of Theoretical Physics, 16(1), 45 (1956), URL http://dx.doi.org/10.1143/ PTP. 16. 45.

[KBM12] Karrasch C., Bardarson J.H. and Moore J.E., Finite-Temperature Dynamical Density Matrix Renormalization Group and the Drude Weight of Spin-1/2 Chains. Phys. Rev. Lett., 108, 227206 (2012), URL http://dx.doi.org/10.1103/PhysRevLett.108.227206. 
[Keh06] Kehrein S., The flow equation approach to many-particle systems, number 217, (Springer Verlag, 2006).

[KG01] Kouwenhoven L. and Glazman L., Revival of the Kondo effect. Phys. World, 14, 33-38 (2001).

$\left[\mathrm{KHB}^{+}\right.$04] Kim Y.J., Hill J.P., Benthien H., Essler F.H.L., Jeckelmann E., Choi H.S., Noh T.W., Motoyama N., Kojima K.M., Uchida S., Casa D. and Gog T., Resonant Inelastic X-Ray Scattering of the Holon-Antiholon Continuum in $\mathrm{SrCuO}_{2}$. Phys. Rev. Lett., 92, 137402 (2004), URL http://dx.doi.org/10.1103/PhysRevLett.92.137402.

[KHFP11] Kalz A., Honecker A., Fuchs S. and Pruschke T., Quantum disordered ground state for hard-core bosons on the frustrated square lattice. Phys. Rev. B, 83, 174519 (2011), URL http://dx.doi.org/10.1103/PhysRevB.83.174519.

[KHM98] Karbach M., Hu K. and Müller G., Introduction to the Bethe ansatz II. Computers in Physics, 12, 565 (1998), URL http://dx.doi.org/10.1063/1.168740.

$\left[\mathrm{KKM}^{+} 08\right] \quad$ Kleine A., Kollath C., McCulloch I.P., Giamarchi T. and Schollwöck U., Spin-charge separation in two-component Bose gases. Phys. Rev. A, 77, 013607 (2008), URL http://dx. doi.org/10.1103/PhysRevA.77.013607.

$\left[\mathrm{KKR}^{+} 06\right] \quad$ Kim B., Koh H., Rotenberg E., Oh S., Eisaki H., Motoyama N., Uchida S., Tohyama T., Maekawa S., Shen Z. et al., Distinct spinon and holon dispersions in photoemission spectral functions from one-dimensional $\mathrm{SrCuO2.} \mathrm{Nat.} \mathrm{Phys.,} \mathrm{2(6),} 397$ (2006), URL http://dx. doi.org/10.1038/nphys316.

[KM96] Kittel C. and McEuen P., Introduction to solid state physics, volume 7, (Wiley New York, 1996).

[KM98] Karbach M. and Müller G., Introduction to the Bethe ansatz I. Computers in Physics, 11 (1998).

[KMB ${ }^{+97] \quad K a r b a c h ~ M ., ~ M u ̈ l l e r ~ G ., ~ B o u g o u r z i ~ A . H ., ~ F l e d d e r j o h a n n ~ A . ~ a n d ~ M u ̈ t t e r ~ K . H ., ~ T w o-s p i n o n ~}$ dynamic structure factor of the one-dimensional $s=$ Heisenberg antiferromagnet. Phys. Rev. B, 55(18), 12510 (1997), URL http://dx.doi.org/10.1103/PhysRevB.55.12510.

[KMS ${ }^{+}$96] Kim C., Matsuura A.Y., Shen Z.X., Motoyama N., Eisaki H., Uchida S., Tohyama T. and Maekawa S., Observation of Spin-Charge Separation in One-Dimensional $\mathrm{SrCu}_{2}$. Phys. Rev. Lett., 77, 4054 (1996), URL http://dx.doi.org/10.1103/PhysRevLett.77.4054.

[KmWW80a] Krishna-murthy H.R., Wilkins J.W. and Wilson K.G., Renormalization-group approach to the Anderson model of dilute magnetic alloys. I. Static properties for the symmetric case. Phys. Rev. B, 21, 1003 (1980), URL http://dx.doi.org/10.1103/PhysRevB.21.1003.

[KmWW80b] Krishna-murthy H.R., Wilkins J.W. and Wilson K.G., Renormalization-group approach to the Anderson model of dilute magnetic alloys. II. Static properties for the asymmetric case. Phys. Rev. B, 21, 1044 (1980), URL http://dx.doi.org/10.1103/PhysRevB.21.1044.

[Kon64] Kondo J., Resistance Minimum in Dilute Magnetic Alloys. Progress of Theoretical Physics, 32(1), 37 (1964), URL http://dx.doi.org/10.1143/PTP.32.37.

$\left[\mathrm{KSD}^{+}\right.$02] Knorr N., Schneider M.A., Diekhöner L., Wahl P. and Kern K., Kondo Effect of Single Co Adatoms on Cu Surfaces. Phys. Rev. Lett., 88, 096804 (2002), URL http://dx.doi.org/ 10.1103/PhysRevLett.88.096804.

[KSZ05] Kollath C., Schollwöck U. and Zwerger W., Spin-Charge Separation in Cold Fermi Gases: A Real Time Analysis. Phys. Rev. Lett., 95, 176401 (2005), URL http://dx.doi.org/ 10.1103/PhysRevLett.95.176401.

[KV04] Kotliar G. and Vollhardt D., Strongly correlated materials: Insights from dynamical meanfield theory. Physics Today, 57(3), 53 (2004), URL http://dx.doi.org/10.1063/1. 1712502.

[KW41] Kramers H.A. and Wannier G.H., Statistics of the Two-Dimensional Ferromagnet. Part II. Phys. Rev., 60, 263 (1941), URL http://dx.doi.org/10.1103/PhysRev.60.263. 
[KW99] Kühner T.D. and White S.R., Dynamical correlation functions using the density matrix renormalization group. Phys. Rev. B, 60(1), 335 (1999), URL http://dx.doi.org/10. 1103/PhysRevB.60.335.

[KWM00] Kühner T.D., White S.R. and Monien H., One-dimensional Bose-Hubbard model with nearest-neighbor interaction. Phys. Rev. B, 61, 12474 (2000), URL http://dx.doi.org/ 10.1103/PhysRevB.61.12474.

[Kö11] Köhler T., The Density Matrix Renormalization Group for the transverse Ising quantum chain: A demonstration program, Thesis, Institut für theoretische Physik, Universität Göttingen (2011).

[Lan58] Landau L.D., Theory of the Fermi liquid. J. Exptl. Theoret. Phys., 35(97) (1958).

[LB04] Limot L. and Berndt R., Kondo effect and surface-state electrons. Applied Surface Science, 237(1-4), 572 (2004), URL http://dx.doi.org/10.1016/j.apsusc.2004.07.023.

[LB05] Landau D. and Binder K., A guide to Monte Carlo simulations in statistical physics, (Cambridge Univ Pr, 2005).

[LCNJ06] Lin C.Y., Castro Neto A.H. and Jones B.A., First-Principles Calculation of the Single Impurity Surface Kondo Resonance. Phys. Rev. Lett., 97, 156102 (2006), URL http:// dx.doi.org/10.1103/PhysRevLett.97.156102.

[Leg06] Leggett A., What do we know about high Tc? Nat. Phys., 2(3), 134 (2006), URL http:// dx.doi.org/10.1038/nphys254.

[LSBD98] Li J., Schneider W.D., Berndt R. and Delley B., Kondo Scattering Observed at a Single Magnetic Impurity. Phys. Rev. Lett., 80, 2893 (1998), URL http://dx. doi .org/10.1103/ PhysRevLett.80.2893.

[Mah00] Mahan G.D., Many-Particle Physics, (Kluwer Academic / Plenum Publishers, 2000), 3rd edition.

[MAVB11] Martins C., Aichhorn M., Vaugier L. and Biermann S., Reduced Effective Spin-Orbital Degeneracy and Spin-Orbital Ordering in Paramagnetic Transition-Metal Oxides: $\mathrm{Sr}_{2} \mathrm{IrO}_{4}$ versus $\mathrm{Sr}_{2} \mathrm{RhO}_{4}$. Phys. Rev. Lett., 107, 266404 (2011), URL http://dx.doi.org/10. 1103/PhysRevLett.107.266404.

[MBB79] Müller G., Beck H. and Bonner J.C., Zero-Temperature Dynamics of the $S=\frac{1}{2}$ Linear Heisenberg Antiferromagnet. Phys. Rev. Lett., 43(1), 75 (1979), URL http://dx.doi. org/10.1103/PhysRevLett.43.75.

[MBB11] Mitchell A.K., Becker M. and Bulla R., Real-space renormalization group flow in quantum impurity systems: Local moment formation and the Kondo screening cloud. Phys. Rev. B, 84, 115120 (2011), URL http://dx.doi.org/10.1103/PhysRevB.84.115120.

[McC07] McCulloch I.P., From density-matrix renormalization group to matrix product states. J. Stat. Mech.: Th. and Exp., 2007(10), P10014 (2007), URL http://dx.doi .org/10.1088/ $1742-5468 / 2007 / 10 /$ P10014.

[MCJ'98] Madhavan V., Chen W., Jamneala T., Crommie M.F. and Wingreen N.S., Tunneling into a Single Magnetic Atom: Spectroscopic Evidence of the Kondo Resonance. Science, 280(5363), 567 (1998), URL http://dx.doi.org/10.1126/science.280.5363.567.

[MG02] McCulloch I.P. and Gulácsi M., The non-Abelian density matrix renormalization group algorithm. EPL, 57(6), 852 (2002), URL http://dx.doi.org/10.1209/epl/ i2002-00393-0.

[MLE00] Manoharan H.C., Lutz C.P. and Eigler D.M., Quantum mirages formed by coherent projection of electronic structure. Nature, 403(6769), 512 (2000), URL http://dx.doi.org/ $10.1038 / 35000508$.

[MM03] Mortimer C. and Müller U., Das Basiswissen der Chemie, (Georg Thieme-Verlag, Stuttgart, 2003). 
[MMN05] Manmana S.R., Muramatsu A. and Noack R.M., Time evolution of one-dimensional Quantum Many Body Systems. AIP Conf. Proc., 789(1), 269 (2005), URL http://dx.doi .org/ 10.1063/1.2080353.

[Mot49] Mott N., The basis of the electron theory of metals, with special reference to the transition metals. Proceedings of the Physical Society. Section A, 62, 416 (1949), URL http://dx. doi.org/10.1088/0370-1298/62/7/303.

$\left[\mathrm{MSH}^{+}{ }^{12}\right] \quad$ Metzner W., Salmhofer M., Honerkamp C., Meden V. and Schönhammer K., Functional renormalization group approach to correlated fermion systems. Rev. Mod. Phys., 84, 299 (2012), URL http://dx.doi.org/10.1103/RevModPhys.84.299.

[MTBB81] Müller G., Thomas H., Beck H. and Bonner J.C., Quantum spin dynamics of the antiferromagnetic linear chain in zero and nonzero magnetic field. Phys. Rev. B, 24, 1429 (1981), URL http://dx.doi.org/10.1103/PhysRevB.24.1429.

[MVC07] Murg V., Verstraete F. and Cirac J.I., Variational study of hard-core bosons in a twodimensional optical lattice using projected entangled pair states. Phys. Rev. A, 75, 033605 (2007), URL http://dx.doi.org/10.1103/PhysRevA.75.033605.

[Nag65] Nagaoka Y., Self-Consistent Treatment of Kondo's Effect in Dilute Alloys. Phys. Rev., 138, A1112 (1965), URL http://dx.doi.org/10.1103/PhysRev.138.A1112.

[NC09] Nevidomskyy A.H. and Coleman P., Kondo Resonance Narrowing in $d$ - and f-Electron Systems. Phys. Rev. Lett., 103, 147205 (2009), URL http://dx.doi.org/10.1103/ PhysRevLett.103.147205.

[NCH10] Nishikawa Y., Crow D.J.G. and Hewson A.C., Renormalized parameters and perturbation theory for an n-channel Anderson model with Hund's rule coupling: Symmetric case. Phys. Rev. B, 82, 115123 (2010), URL http://dx.doi.org/10.1103/PhysRevB.82.115123.

[Nis99] Nishiyama Y., Numerical analysis of the dissipative two-state system with the densitymatrix Hilbert-space-reduction algorithm. Eur. Phys. J. B, 12(4), 547 (1999), URL http: // dx.doi.org/10.1007/s100510051037.

[NJ04] Nishimoto S. and Jeckelmann E., Density-matrix renormalization group approach to quantum impurity problems. J. Phys.: Cond. Matt., 16, 613 (2004), URL http://dx.doi.org/ 10.1088/0953-8984/16/4/010.

$\left[\mathrm{NKB}^{+} 08\right] \quad$ Néel N., Kröger J., Berndt R., Wehling T.O., Lichtenstein A.I. and Katsnelson M.I., Controlling the Kondo Effect in $\mathrm{CoCu}_{n}$ Clusters Atom by Atom. Phys. Rev. Lett., 101, 266803 (2008), URL http://dx.doi.org/10.1103/PhysRevLett.101.266803.

[NKL $\left.{ }^{+} 07\right] \quad$ Néel N., Kröger J., Limot L., Palotas K., Hofer W.A. and Berndt R., Conductance and Kondo Effect in a Controlled Single-Atom Contact. Phys. Rev. Lett., 98, 016801 (2007), URL http://dx.doi.org/10.1103/PhysRevLett.98.016801.

[NM05] Noack R.M. and Manmana S.R., Diagonalization- and Numerical Renormalization-GroupBased Methods for Interacting Quantum Systems. AIP Conf. Proc., 789(1), 93 (2005), URL http://dx.doi.org/10.1063/1.2080349.

[Nol05] Nolting W., Viel-Teilchen-Theorie, (Springer, 2005), 6th edition.

[Noz74] Nozières P., A "Fermi-liquid" description of the Kondo problem at low temperatures. Journal of Low Temperature Physics, 17(1), 31 (1974), URL http://dx.doi.org/10.1007/ BF00654541.

[OAAY01] Okunishi K., Akutsu Y., Akutsu N. and Yamamoto T., Universal relation between the dispersion curve and the ground-state correlation length in one-dimensional antiferromagnetic quantum spin systems. Phys. Rev. B, 64, 104432 (2001), URL http://dx.doi.org/10. 1103/PhysRevB .64.104432.

[OlR95] Östlund S. and Rommer S., Thermodynamic Limit of Density Matrix Renormalization. Phys. Rev. Lett., 75(19), 3537 (1995), URL http://dx.doi.org/10.1103/PhysRevLett. 75.3537. 
[Onn11] Onnes H.K., The disappearance of the resistivity of mercury. Comm. Leiden, 122((b)) (1911).

[Osb12] Osborne T.J., Hamiltonian complexity. Reports on Progress in Physics, 75(2), 022001 (2012), URL http://dx.doi.org/10.1088/0034-4885/75/2/022001.

$\left[\mathrm{OTvB}^{+} 08\right] \quad$ Otte A.F., Ternes M., von Bergmann K., Loth S., Brune H., Lutz C.P., Hirjibehedin C.F. and Heinrich A.J., The role of magnetic anisotropy in the Kondo effect. Nat. Phys., 4(11), 847 (2008), URL http://dx.doi.org/10.1038/nphys1072.

[Pap86] Papaconstantopoulos D.A., Handbook of The Band Structure of Elemental Solids, (Plenum Press, 1986).

[PB05] Pruschke T. and Bulla R., Hund's coupling and the metal-insulator transition in the twoband Hubbard model. Eur. Phys. J. B, 44, 217 (2005), URL http://dx.doi .org/10.1140/ epjb/e2005-00117-4.

[PDSB85] Patthey F., Delley B., Schneider W.D. and Baer Y., Low-Energy Excitations in $\alpha$ - and $\gamma-$ Ce Observed by Photoemission. Phys. Rev. Lett., 55, 1518 (1985), URL http://dx. doi.org/10.1103/PhysRevLett.55.1518.

[PEDC05] Plenio M.B., Eisert J., Dreißig J. and Cramer M., Entropy, Entanglement, and Area: Analytical Results for Harmonic Lattice Systems. Phys. Rev. Lett., 94, 060503 (2005), URL http://dx.doi.org/10.1103/PhysRevLett.94.060503.

[Pet11] Peters R., Spectral functions for single- and multi-impurity models using density matrix renormalization group. Phys. Rev. B, 84, 075139 (2011), URL http://dx.doi.org/10. 1103/PhysRevB.84.075139.

[PG89] Pruschke T. and Grewe N., The Anderson model with finite Coulomb repulsion. Zeit. f. Phys. B Cond. Mat., 74, 439 (1989), URL http://dx.doi.org/10.1007/BF01311391.

[PHV12] Pirvu B., Haegeman J. and Verstraete F., Matrix product state based algorithm for determining dispersion relations of quantum spin chains with periodic boundary conditions. Phys. Rev. B, 85, 035130 (2012), URL http://dx.doi.org/10.1103/PhysRevB.85.035130.

[PLAH08] Pereira R.G., Laflorencie N., Affleck I. and Halperin B.I., Kondo screening cloud and charge staircase in one-dimensional mesoscopic devices. Phys. Rev. B, 77(12), 125327 (2008), URL http://dx.doi.org/10.1103/PhysRevB.77.125327.

[PMCV10] Pirvu B., Murg V., Cirac J.I. and Verstraete F., Matrix product operator representations. New Journal of Physics, 12(2), 025012 (2010), URL http://dx. doi .org/10.1088/ $1367-2630 / 12 / 2 / 025012$.

[Pot12] Potthoff M., Self-Energy-Functional Theory, in Strongly Correlated Systems (edited by A. Avella and F. Mancini), volume 171 of Springer Series in Solid-State Sciences, pages 303-339, (Springer Berlin Heidelberg, 2012).

[PPA06] Peters R., Pruschke T. and Anders F.B., Numerical renormalization group approach to Green's functions for quantum impurity models. Phys. Rev. B, 74, 245114 (2006), URL http://dx.doi.org/10.1103/PhysRevB.74.245114.

[PRSB99] Pati S.K., Ramasesha S., Shuai Z. and Brédas J.L., Dynamical nonlinear optical coefficients from the symmetrized density-matrix renormalization-group method. Phys. Rev. B, 59, 14827 (1999), URL http://dx.doi.org/10.1103/PhysRevB.59.14827.

[Pru08] Prueser H., Scanning Tunnelling Spectroscopy of Subsurface Magnetic Atoms, Thesis, Georg-August-Universität Göttingen (2008).

[PSC ${ }^{+}$06] Pereira R.G., Sirker J., Caux J.S., Hagemans R., Maillet J.M., White S.R. and Affleck I., Dynamical Spin Structure Factor for the Anisotropic Spin-1/2 Heisenberg Chain. Phys. Rev. Lett., 96, 257202 (2006), URL http://dx.doi.org/10.1103/PhysRevLett. 96.257202.

[PTVF92] Press W.H., Teukolsky S.A., Vetterling W.T. and Flan B.P., Numerical Recipes, (Cambridge University Press, 1992). 
[PWA08] Pereira R.G., White S.R. and Affleck I., Exact Edge Singularities and Dynamical Correlations in Spin-1/2 Chains. Phys. Rev. Lett., 100(2), 027206 (2008), URL http://dx.doi. org/10.1103/PhysRevLett.100.027206.

[PWA09] Pereira R.G., White S.R. and Affleck I., Spectral function of spinless fermions on a onedimensional lattice. Phys. Rev. B, 79, 165113 (2009), URL http://dx.doi.org/10.1103/ PhysRevB.79.165113.

[PWD $\left.{ }^{+} 11\right] \quad$ Prüser H., Wenderoth M., Dargel P.E., Weismann A., Peters R., Pruschke T. and Ulbrich R.G., Long-range Kondo signature of a single magnetic impurity. Nat. Phys., 7(3), 203 (2011), URL http://dx.doi.org/10.1038/nphys1876.

[PWWU12] Prüser H., Wenderoth M., Weismann A. and Ulbrich R.G., Mapping Itinerant Electrons around Kondo Impurities. Phys. Rev. Lett., 108, 166604 (2012), URL http://dx.doi. org/10.1103/PhysRevLett.108.166604.

[QWW $\left.{ }^{+} 04\right]$ Quaas N., Wenderoth M., Weismann A., Ulbrich R.G. and Schönhammer K., Kondo resonance of single Co atoms embedded in Cu(111). Phys. Rev. B, 69, 201103 (2004), URL http://dx.doi.org/10.1103/PhysRevB.69.201103.

[Ray70] Rayleigh J., In Finding the Correction for the Open End of an Organ-Pipe. Philosophical Transactions, 161 (1870).

[Req06] Requardt M., Entanglement-Entropy for Groundstates, Low-lying and Highly Excited Eigenstates of General (Lattice) Hamiltonians (2006), URL arxiv:hep-th/0605142v4.

[Rit08] Ritz W., Über eine neue Methode zur Lösung gewisser Variationsprobleme der mathematischen Physik. Journal für die reine und angewandte Mathematik, 135 (1908).

[RK54] Ruderman M.A. and Kittel C., Indirect Exchange Coupling of Nuclear Magnetic Moments by Conduction Electrons. Phys. Rev., 96, 99 (1954), URL http://dx.doi.org/10.1103/ PhysRev. 96.99.

[RPK ${ }^{+96] \quad R a m a s e s h a ~ S ., ~ P a t i ~ S . K ., ~ K r i s h n a m u r t h y ~ H . R ., ~ S h u a i ~ Z . ~ a n d ~ B r e ́ d a s ~ J . L ., ~ S y m m e t r i z e d ~}$ density-matrix renormalization-group method for excited states of Hubbard models. Phys. Rev. B, 54(11), 7598 (1996), URL http://dx.doi.org/10.1103/PhysRevB.54.7598.

[RTC $\left.{ }^{+} 11\right] \quad$ Rule K.C., Tennant D.A., Caux J.S., Gibson M.C.R., Telling M.T.F., Gerischer S., Süllow S. and Lang M., Dynamics of azurite $\mathrm{Cu}_{3}\left(\mathrm{CO}_{3}\right)_{2}(\mathrm{OH})_{2}$ in a magnetic field as determined by neutron scattering. Phys. Rev. B, 84, 184419 (2011), URL http://dx.doi.org/10. 1103/PhysRevB . 84.184419.

[RU05] Raas C. and Uhrig G.S., Spectral densities from dynamic density-matrix renormalization. Eur. Phys. J. B, 45, 293 (2005), URL http://dx.doi.org/10.1140/epjb/ e2005-00194-3.

[RUA04] Raas C., Uhrig G.S. and Anders F.B., High-energy dynamics of the single-impurity Anderson model. Phys. Rev. B, 69(4), 041102 (2004), URL http://dx.doi.org/10.1103/ PhysRevB.69.041102.

[SA96] Sørensen E.S. and Affleck I., Scaling theory of the Kondo screening cloud. Phys. Rev. B, 53(14), 9153 (1996), URL http://dx.doi.org/10.1103/PhysRevB.53.9153.

[SA03] Simon P. and Affleck I., Kondo screening cloud effects in mesoscopic devices. Phys. Rev. B, 68, 115304 (2003), URL http://dx.doi.org/10.1103/PhysRevB.68.115304.

[SA05] Sørensen E.S. and Affleck I., Kondo Screening Cloud Around a Quantum Dot: Large-Scale Numerical Results. Phys. Rev. Lett., 94(8), 086601 (2005), URL http://dx.doi .org/10. 1103/PhysRevLett.94.086601.

[SAB $\left.{ }^{+} 79\right] \quad$ Steglich F., Aarts J., Bredl C.D., Lieke W., Meschede D., Franz W. and Schäfer H., Superconductivity in the Presence of Strong Pauli Paramagnetism: $\mathrm{CeCu}_{2} \mathrm{Si}_{2}$. Phys. Rev. Lett., 43, 1892 (1979), URL http://dx.doi.org/10.1103/PhysRevLett.43.1892.

[Sch26a] Schrödinger E., Quantisierung als Eigenwertproblem I. Ann. d. Physik, 384(4), 361 (1926), URL http://dx.doi.org/10.1002/andp.19263840404. 
[Sch26b] Schrödinger E., Quantisierung als Eigenwertproblem II. Ann. d. Physik, 385(13), 437 (1926), URL http://dx.doi.org/10.1002/andp.19263851302.

[Sch48a] Schwinger J., On Quantum-Electrodynamics and the Magnetic Moment of the Electron. Phys. Rev., 73, 416 (1948), URL http://dx.doi.org/10.1103/PhysRev.73.416.

[Sch48b] Schwinger J., Quantum Electrodynamics. I. A Covariant Formulation. Phys. Rev., 74, 1439 (1948), URL http://dx.doi.org/10.1103/PhysRev.74.1439.

[Sch49] Schwinger J., Quantum Electrodynamics. III. The Electromagnetic Properties of the Electron-Radiative Corrections to Scattering. Phys. Rev., 76, 790 (1949), URL http://dx. doi.org/10.1103/PhysRev.76.790.

[Sch76] Schönhammer K., Variational Ansatz for the Anderson model of chemisorption. Phys. Rev. B, 13, 4336 (1976), URL http://dx.doi.org/10.1103/PhysRevB.13.4336.

[Sch95] Schulz H.J., Fermi liquids and non-Fermi liquids, in Proceedings of Les Houches Summer School LXI (edited by J.P.J.Z.J. E. Akkermans G. Montambaux), (Elsevier, 1995).

[Sch04] Schmitteckert P., Nonequilibrium electron transport using the density matrix renormalization group method. Phys. Rev. B, 70, 121302 (2004), URL http://dx.doi.org/10.1103/ PhysRevB.70.121302.

[Sch05a] Schollwöck U., Time-dependent Density-Matrix Renormalization-Group Methods. J. Phys. Soc. Jpn., 74S(Supplement), 246 (2005), URL http://dx.doi.org/10.1143/JPS JS.74S . 246.

[Sch05b] Schollwöck U., The density-matrix renormalization group. Rev. Mod. Phys., 77, 259 (2005), URL http://dx.doi.org/10.1103/RevModPhys.77.259.

[Sch11] Schollwöck U., The density-matrix renormalization group in the age of matrix product states. Ann. Phys., 326, 96 (2011), URL http://dx.doi.org/10.1016/j.aop.2010.09. 012.

[SGSJ90] Silver R.N., Gubernatis J.E., Sivia D.S. and Jarrell M., Spectral densities of the symmetric Anderson model. Phys. Rev. Lett., 65, 496 (1990), URL http://dx.doi.org/10.1103/ PhysRevLett.65.496.

[Sha08] Shankar R., Colloquium : Chaotic quantum dots with strongly correlated electrons. Rev. Mod. Phys., 80, 379 (2008), URL http://dx.doi.org/10.1103/RevModPhys.80.379.

[Shu95] Shull C.G., Early development of neutron scattering. Rev. Mod. Phys., 67, 753 (1995), URL http://dx.doi.org/10.1103/RevModPhys.67.753.

[SK83] Smith J. and Kmetko E., Magnetism or bonding: A nearly periodic table of transition elements. Journal of the Less Common Metals, 90(1), 83 (1983), URL http://dx.doi. org/10.1016/0022-5088(83)90119-4.

[SK05] Sirker J. and Klümper A., Real-time dynamics at finite temperature by the density-matrix renormalization group: A path-integral approach. Phys. Rev. B, 71, 241101 (2005), URL http://dx.doi.org/10.1103/PhysRevB.71.241101.

[SP53] Stückelberg E. and Petermann A., Normalization of constants in the quanta theory. Helvetica Physica Acta, 26, 499 (1953).

[Sre93] Srednicki M., Entropy and area. Phys. Rev. Lett., 71, 666 (1993), URL http://dx.doi. org/10.1103/PhysRevLett.71.666.

[SS10] Si Q. and Steglich F., Heavy fermions and quantum phase transitions. Science, 329(5996), 1161 (2010), URL http://dx.doi.org/10.1126/science.1191195.

[SSK89] Sakai O., Shimizu Y. and Kasuya T., Single-Particle and Magnetic Excitation Spectra of Degenerate Anderson Model with Finite f-f Coulomb Interaction. J. Phys. Soc. Jpn., 58(10), 3666 (1989), URL http://dx.doi.org/10.1143/JPSJ.58.3666.

[SSK90] Shimizu Y., Sakai O. and Kasuya T., Theory of the Kondo effect for Sm-like systems. Phys. B: Cond. Mat., 163, 401 (1990), URL http://dx.doi.org/10.1016/ 0921-4526 (90) 90224-I. 
[SSMS09] Smerat S., Schollwöck U., McCulloch I.P. and Schoeller H., Quasiparticles in the Kondo lattice model at partial fillings of the conduction band using the density matrix renormalization group. Phys. Rev. B, 79, 235107 (2009), URL http://dx.doi.org/10.1103/PhysRevB. 79.235107.

[ST10] Sota S. and Tohyama T., Density matrix renormalization group study of optical conductivity in the one-dimensional Mott insulator $\mathrm{Sr}_{2} \mathrm{CuO}_{3}$. Phys. Rev. B, 82, 195130 (2010), URL http://dx.doi.org/10.1103/PhysRevB.82.195130.

[Ste84] Stewart G.R., Heavy-fermion systems. Rev. Mod. Phys., 56, 755 (1984), URL http://dx. doi.org/10.1103/RevModPhys.56.755.

$\left[\mathrm{STW}^{+} 12\right] \quad$ Surer B., Troyer M., Werner P., Wehling T.O., Läuchli A.M., Wilhelm A. and Lichtenstein A.I., Multiorbital Kondo physics of Co in Cu hosts. Phys. Rev. B, 85, 085114 (2012), URL http://dx.doi.org/10.1103/PhysRevB.85.085114.

[Suh65] Suhl H., Dispersion Theory of the Kondo Effect. Phys. Rev., 138, A515 (1965), URL http://dx.doi.org/10.1103/PhysRev.138.A515.

[SW66] Schrieffer J.R. and Wolff P.A., Relation between the Anderson and Kondo Hamiltonians. Phys. Rev., 149(2), 491 (1966), URL http://dx.doi.org/10.1103/PhysRev.149.491.

[SWvD08] Saberi H., Weichselbaum A. and von Delft J., Matrix-product-state comparison of the numerical renormalization group and the variational formulation of the density-matrix renormalization group. Phys. Rev. B, 78, 035124 (2008), URL http://dx.doi.org/10.1103/ PhysRevB.78.035124.

[Sym71] Symanzik K., Small-distance-behaviour analysis and Wilson expansions. Comm. in Math. Phys., 23, 49 (1971), URL http://dx.doi.org/10.1007/BF01877596.

[SZV10] Singh S., Zhou H.Q. and Vidal G., Simulation of one-dimensional quantum systems with a global SU(2) symmetry. New Journal of Physics, 12(3), 033029 (2010), URL http://dx. doi.org/10.1088/1367-2630/12/3/033029.

[TH83] Tersoff J. and Hamann D.R., Theory and Application for the Scanning Tunneling Microscope. Phys. Rev. Lett., 50(25), 1998 (1983), URL http://dx.doi.org/10.1103/ PhysRevLett.50.1998.

[TH85] Tersoff J. and Hamann D.R., Theory of the scanning tunneling microscope. Phys. Rev. B, 31(2), 805 (1985), URL http://dx.doi.org/10.1103/PhysRevB.31.805.

[THS09] Ternes M., Heinrich A.J. and Schneider W.D., Spectroscopic manifestations of the Kondo effect on single adatoms. J. Phys.: Cond. Matt., (5), 053001 (2009), URL http://dx.doi. org/10.1088/0953-8984/21/5/053001.

[Tie12] Tiegel A., Optical Properties of the 1D Hubbard Model studied with DMRG, Thesis, GeorgAugust-Universität Göttingen (2012).

[Tom46] Tomonaga S., On a Relativistically Invariant Formulation of the Quantum Theory of Wave Fields. Progress of Theoretical Physics, 1(2), 27 (1946), URL http://dx.doi.org/10. 1143/PTP.1.27.

[TRR ${ }^{+}$09] Thielemann B., Rüegg C., Rønnow H.M., Läuchli A.M., Caux J.S., Normand B., Biner D., Krämer K.W., Güdel H.U., Stahn J., Habicht K., Kiefer K., Boehm M., McMorrow D.F. and Mesot J., Direct Observation of Magnon Fractionalization in the Quantum Spin Ladder. Phys. Rev. Lett., 102, 107204 (2009), URL http://dx.doi.org/10.1103/PhysRevLett. 102.107204.

[TW83] Tsvelick A.M. and Wiegmann P.B., Exact solution of the Anderson model. II. Thermodynamic properties at finite temperatures. J. Phys. C: Sol. Stat. Phys., 16(12), 2321 (1983), URL http://dx.doi.org/10.1088/0022-3719/16/12/018.

[UKSZ00] Újsághy O., Kroha J., Szunyogh L. and Zawadowski A., Theory of the Fano Resonance in the STM Tunneling Density of States due to a Single Kondo Impurity. Phys. Rev. Lett., 85, 2557 (2000), URL http://dx.doi.org/10.1103/PhysRevLett.85.2557, cond-mat/ 0005166 . 
[VC04] Verstraete F. and Cirac J.I., Renormalization algorithms for Quantum-Many Body Systems in two and higher dimensions (2004), URL arXiv: cond-mat/0407066v1.

[VC06] Verstraete F. and Cirac J.I., Matrix product states represent ground states faithfully. Phys. Rev. B, 73, 094423 (2006), URL http://dx.doi.org/10.1103/PhysRevB.73.094423.

[VGRC04] Verstraete F., García-Ripoll J.J. and Cirac J.I., Matrix Product Density Operators: Simulation of Finite-Temperature and Dissipative Systems. Phys. Rev. Lett., 93(20), 207204 (2004), URL http://dx.doi.org/10.1103/PhysRevLett.93.207204.

[Vid03] Vidal G., Efficient Classical Simulation of Slightly Entangled Quantum Computations. Phys. Rev. Lett., 91, 147902 (2003), URL http://dx.doi.org/10.1103/PhysRevLett. 91.147902 .

[Vid04] Vidal G., Efficient Simulation of One-Dimensional Quantum Many-Body Systems. Phys. Rev. Lett., 93(4), 040502 (2004), URL http://dx.doi.org/10.1103/PhysRevLett.93. 040502.

[Vid07] Vidal G., Entanglement Renormalization. Phys. Rev. Lett., 99, 220405 (2007), URL http://dx.doi.org/10.1103/PhysRevLett.99.220405.

[Vid08] Vidal G., Class of Quantum Many-Body States That Can Be Efficiently Simulated. Phys. Rev. Lett., 101, 110501 (2008), URL http://dx.doi.org/10.1103/PhysRevLett.101. 110501.

[VLRK03] Vidal G., Latorre J.I., Rico E. and Kitaev A., Entanglement in Quantum Critical Phenomena. Phys. Rev. Lett., 90, 227902 (2003), URL http://dx.doi.org/10.1103/ PhysRevLett. 90 .227902.

[VMC08] Verstraete F., Murg V. and Cirac J., Matrix product states, projected entangled pair states, and variational renormalization group methods for quantum spin systems. Advances in Physics, 57(2), 143 (2008), URL http://dx.doi.org/10.1080/14789940801912366.

[vP09] Žitko R. and Pruschke T., Energy resolution and discretization artifacts in the numerical renormalization group. Phys. Rev. B, 79, 085106 (2009), URL http://dx.doi.org/10. 1103/PhysRevB.79.085106.

[VPC04] Verstraete F., Porras D. and Cirac J.I., Density Matrix Renormalization Group and Periodic Boundary Conditions: A Quantum Information Perspective. Phys. Rev. Lett., 93(22), 227205 (2004), URL http://dx.doi.org/10.1103/PhysRevLett.93.227205.

[Ž07] Žitko R., Many-particle effects in resonant tunneling of electrons through nanostructures, $\mathrm{PhD}$ Thesis, University of Ljubljana (2007).

[WDS $\left.{ }^{+} 04\right] \quad$ Wahl P., Diekhöner L., Schneider M.A., Vitali L., Wittich G. and Kern K., Kondo Temperature of Magnetic Impurities at Surfaces. Phys. Rev. Lett., 93, 176603 (2004), URL http://dx.doi.org/10.1103/PhysRevLett.93.176603.

[WDSK08] Wahl P., Diekhoner L., Schneider M.A. and Kern K., Background removal in scanning tunneling spectroscopy of single atoms and molecules on metal surfaces. Rev. Sci. Instrum., 79(4), 043104 (2008), URL http://dx.doi.org/10.1063/1.2907533.

[Weg94] Wegner F., Flow-equations for Hamiltonians. Ann. d. Physik, 506(2), 77 (1994), URL http://dx.doi.org/10.1002/andp.19945060203.

[Wei08] Weismann A., Scanning Tunnelling Spectroscopy of Subsurface Magnetic Atoms in Copper, Electron Focusing and Kondo Effects, PhD Thesis, Georg-August-Universität Göttingen (2008).

[Wei11] Weichselbaum A., Discarded weight and entanglement spectra in the numerical renormalization group. Phys. Rev. B, 84, 125130 (2011), URL http://dx.doi.org/10.1103/ PhysRevB.84.125130.

[WF04] White S.R. and Feiguin A.E., Real-Time Evolution Using the Density Matrix Renormalization Group. Phys. Rev. Lett., 93, 076401 (2004), URL http://dx.doi.org/10.1103/ PhysRevLett . 93.076401. 
[Whi92] White S.R., Density matrix formulation for quantum renormalization groups. Phys. Rev. Lett., 69(19), 2863 (1992), URL http://dx.doi.org/10.1103/PhysRevLett.69.2863.

[Whi93] White S.R., Density-matrix algorithms for quantum renormalization groups. Phys. Rev. B, 48(14), 10345 (1993), URL http://dx.doi.org/10.1103/PhysRevB.48.10345.

[Whi96] White S.R., Spin Gaps in a Frustrated Heisenberg Model for $\mathrm{CaV}_{4} \mathrm{O}_{9}$. Phys. Rev. Lett., 77(17), 3633 (1996), URL http://dx.doi.org/10.1103/PhysRevLett.77.3633.

[Whi05] White S.R., Density matrix renormalization group algorithms with a single center site. Phys. Rev. B, 72, 180403 (2005), URL http://dx.doi.org/10.1103/PhysRevB.72. 180403.

[Wie81] Wiegmann P.B., Exact solution of the s-d exchange model (Kondo problem). J. Phys. C: Sol. Stat. Phys., 14, 1463 (1981), URL http://dx.doi.org/10.1088/0022-3719/14/10/ 014.

[Wie94] Wiesendanger R., Scanning probe microscopy and spectroscopy: methods and applications, (Cambridge University Press, 1994).

[Wil75] Wilson K.G., The renormalization group: Critical phenomena and the Kondo problem. Rev. Mod. Phys., 47(4), 773 (1975), URL http://dx.doi.org/10.1103/RevModPhys .47.773.

[Wil83] Wilson K.G., The renormalization group and critical phenomena. Rev. Mod. Phys., 55, 583 (1983), URL http://dx.doi.org/10.1103/RevModPhys.55.583.

[WN92] White S.R. and Noack R.M., Real-space quantum renormalization groups. Phys. Rev. Lett., 68, 3487 (1992), URL http://dx.doi.org/10.1103/PhysRevLett.68.3487.

[WT83] Wiegmann P.B. and Tsvelik A.M., Exact solution of the Anderson model: I. J. Phys. C: Sol. Stat. Phys., 16, 2281 (1983), URL http://dx.doi.org/10.1088/0022-3719/16/12/ 017.

[WvD07] Weichselbaum A. and von Delft J., Sum-Rule Conserving Spectral Functions from the Numerical Renormalization Group. Phys. Rev. Lett., 99, 076402 (2007), URL http://dx. doi.org/10.1103/PhysRevLett.99.076402.

[WVS ${ }^{+}$09] Weichselbaum A., Verstraete F., Schollwöck U., Cirac J.I. and von Delft J., Variational matrix-product-state approach to quantum impurity models. Phys. Rev. B, 80(16), 165117 (2009), URL http://dx.doi.org/10.1103/PhysRevB.80.165117.

[WWAF06] Weisse A., Wellein G., Alvermann A. and Fehske H., The kernel polynomial method. Rev. Mod. Phys., 78(1), 275 (2006), URL http://dx.doi.org/10.1103/RevModPhys.78. 275.

[WWL ${ }^{+}$09] Weismann A., Wenderoth M., Lounis S., Zahn P., Quaas N., Ulbrich R.G., Dederichs P.H. and Blugel S., Seeing the Fermi Surface in Real Space by Nanoscale Electron Focusing. Science, 323(5918), 1190 (2009), URL http://dx.doi.org/10.1126/science.1168738.

[WZDX10] Wang L., Zhuang J., Dai X. and Xie X., An Impurity Solver Using the Time-Dependent Variational Matrix Product State Approach. arXiv:1001.2943 (2010).

[Yam75a] Yamada K., Perturbation Expansion for the Anderson Hamiltonian. II. Progress of Theoretical Physics, 53(4), 970 (1975), URL http://dx.doi.org/10.1143/PTP.53.970.

[Yam75b] Yamada K., Perturbation Expansion for the Anderson Hamiltonian. IV. Progress of Theoretical Physics, 54(2), 316 (1975), URL http://dx.doi.org/10.1143/PTP.54.316.

[YH00] Yu W. and Haas S., Dynamical properties of spin-orbital chains in a magnetic field. Phys. Rev. B, 63, 024423 (2000), URL http://dx.doi.org/10.1103/PhysRevB.63.024423.

[Yos57a] Yosida K., Anomalous Electrical Resistivity and Magnetoresistance Due to an $s-d$ Interaction in Cu-Mn Alloys. Phys. Rev., 107, 396 (1957), URL http://dx.doi.org/10. 1103/PhysRev.107.396.

[Yos57b] Yosida K., Magnetic Properties of Cu-Mn Alloys. Phys. Rev., 106, 893 (1957), URL http://dx.doi.org/10.1103/PhysRev.106.893. 
[YY70] Yosida K. and Yamada K., Perturbation Expansion for the Anderson Hamiltonian. Progress of Theoretical Physics Supplement, 46, 244 (1970), URL http://dx.doi.org/10.1143/ PTPS. 46.244.

[YY75] Yosida K. and Yamada K., Perturbation Expansion for the Anderson Hamiltonian. III. Progress of Theoretical Physics, 53(5), 1286 (1975), URL http://dx.doi.org/10.1143/ PTP. 53.1286.

[Zen51] Zener C., Interaction Between the d Shells in the Transition Metals. Phys. Rev., 81, 440 (1951), URL http://dx.doi.org/10.1103/PhysRev.81.440.

[ZJW99] Zhang C., Jeckelmann E. and White S.R., Dynamical properties of the one-dimensional Holstein model. Phys. Rev. B, 60, 14092 (1999), URL http://dx.doi.org/10.1103/ PhysRevB .60.14092.

[ZLC ${ }^{+}$05] Zhao A., Li Q., Chen L., Xiang H., Wang W., Pan S., Wang B., Xiao X., Yang J., Hou J.G. and Zhu Q., Controlling the Kondo Effect of an Adsorbed Magnetic Ion Through Its Chemical Bonding. Science, 309(5740), 1542 (2005), URL http://dx.doi.org/10.1126/ science.1113449. 


\section{Lebenslauf}

Name: Piet E. Dargel

Staatsangehörigkeit: deutsch

02.08.1982 geboren in Bremen

09/1989 - 06/2002 Besuch von Grundschule und Gymnasium (Grundschule Bunnsackerweg, Schulzentrum Habenhausen, Gymnasium Obervieland)

06/2002 Erwerb der allgemeinen Hochschulreife

10/2003 - 04/2009 Studium an der Georg-August-Universität Göttingen im Studiengang Physik

10/2005 Erwerb des Vordiploms im Studiengang Physik an der Universität Göttingen

08/2006 - 07/2007 Studium der Physik an der University of California, San Diego

04/2009 Erwerb des Hochschulgrades Diplom-Physiker an der Universität Göttingen

07/2009 - 10/2012 Arbeit an der vorliegenden Dissertation bei Prof. Dr. Thomas Pruschke an der Universität Göttingen 